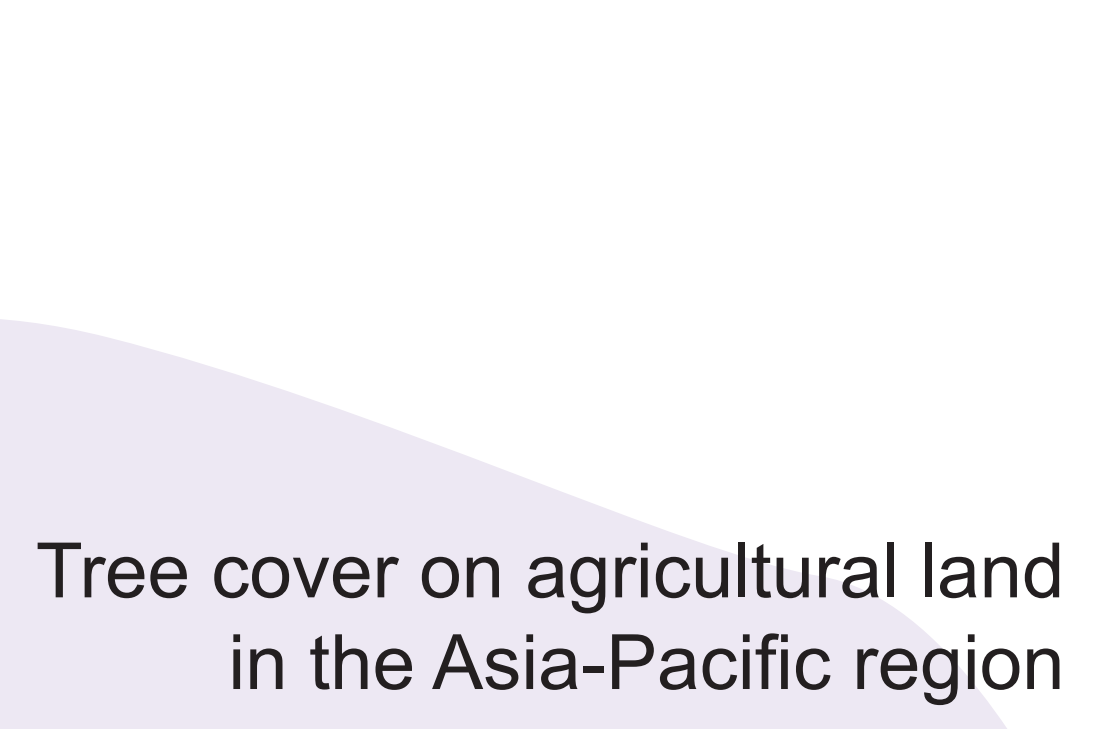

Robert J Zomer, Ingrid Öborn and Jianchu Xu 



\title{
Tree cover on agricultural land in the Asia-Pacific region
}

\author{
Robert J Zomer, Ingrid Öborn and Jianchu Xu
}

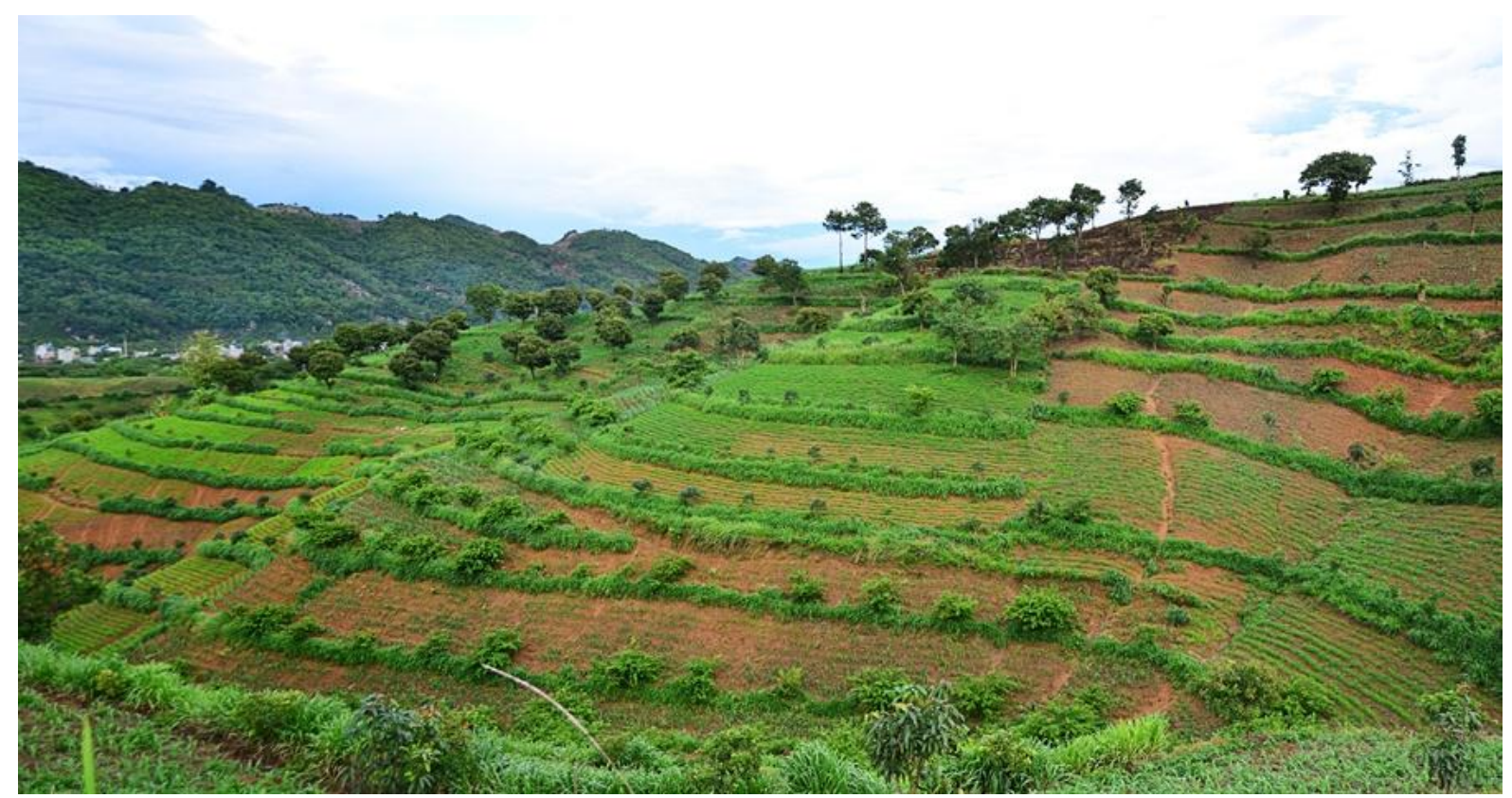




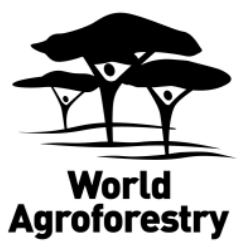

Correct citation:

Zomer R, Öborn I, Xu J. 2019. Tree cover on agricultural land in the Asia-Pacific region. Working Paper 294. Bogor, Indonesia: World Agroforestry (ICRAF) Southeast Asia Regional Program. DOI: http://dx.doi.org/10.5716/WP19005.PDF

Titles in the Working Paper series aim to disseminate interim results on agroforestry research and practices, and stimulate feedback from the scientific community. Other publication series from the World Agroforestry Centre include: Technical Manuals, Occasional Papers and the Trees for Change Series.

Published by World Agroforestry (ICRAF) Southeast Asia Regional Program

Jalan CIFOR, Situ Gede, Sindang Barang

Bogor 16115, Jawa Barat, Indonesia

Tel: +62 2518625415

Email: icraf-indonesia@cgiar.org

Website: www.worlagroforestry.org/region/southeast asia

(C) World Agroforestry 2019

Working Paper 294

Cover photo: Agroforestry with contour planting in Northwest Viet Nam. Photo: World Agroforestry/Mai Phuong Nguyen

The views expressed in this publication are those of the author(s) and not necessarily those of the World Agroforestry.

Articles appearing in this publication may be quoted or reproduced without charge, provided the source is acknowledged.

All images remain the sole property of their source and may not be used for any purpose without written permission from the source. 


\section{Table of contents}

Tree cover on agricultural land in the Asia-Pacific region ........................................................

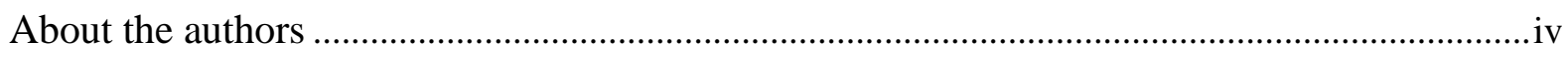

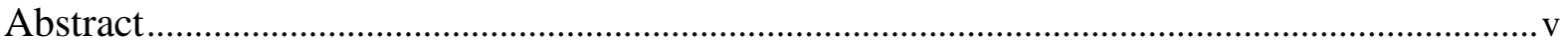

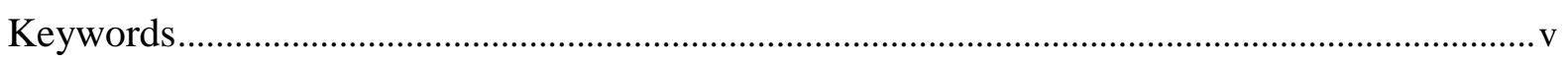

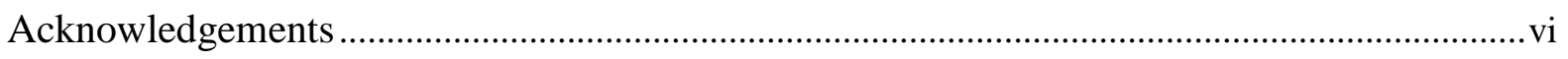

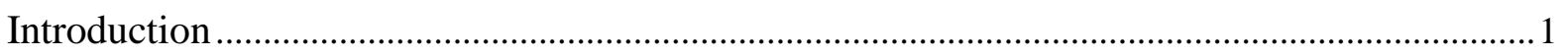

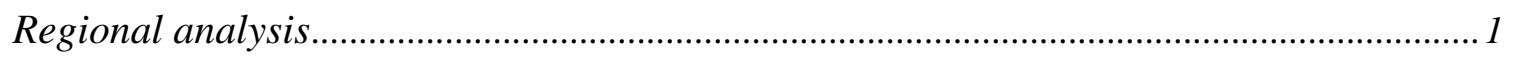

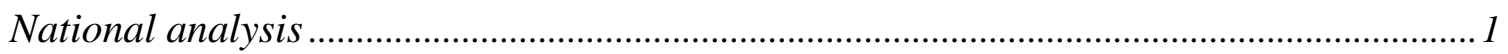

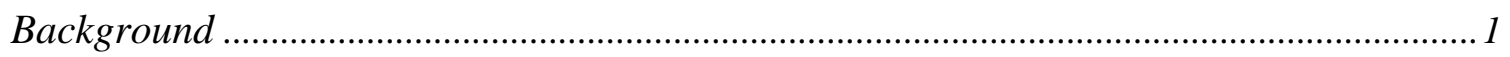

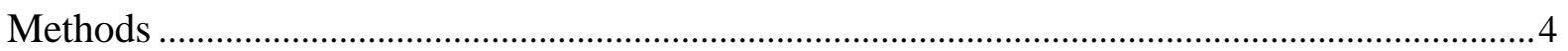

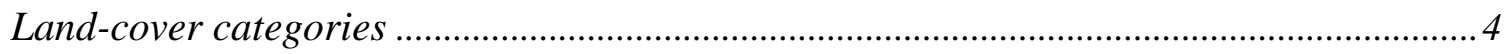

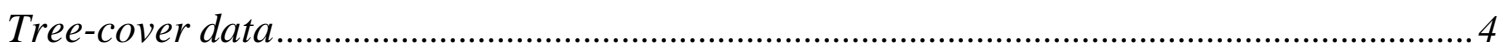

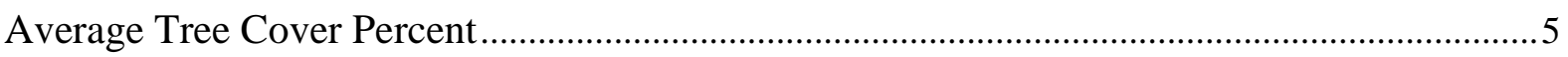

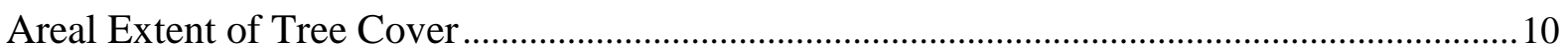

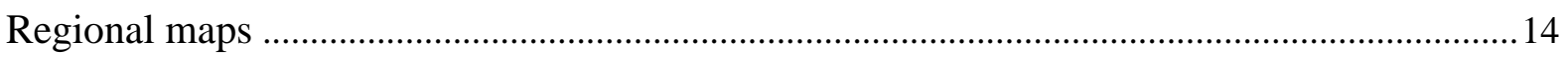

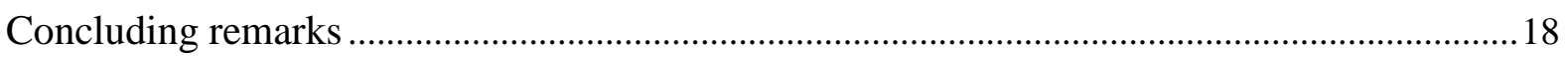

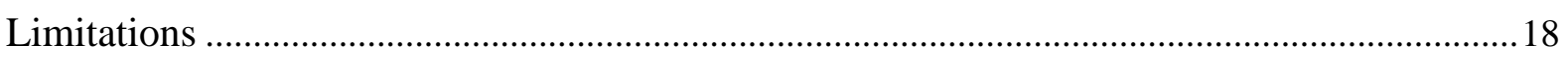

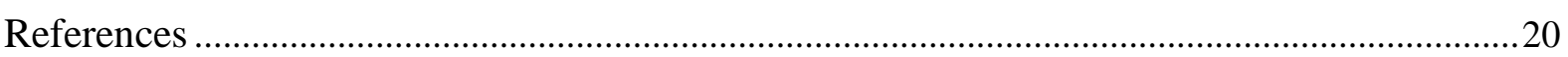

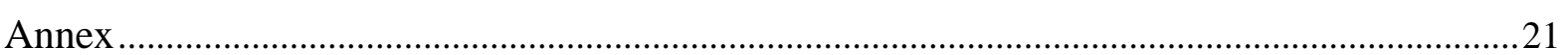




\section{List of figures}

Figure 1. Tree cover on agricultural land, globally, 2000 and 2010 3

Figure 2. Percent tree cover on agricultural land in 2010 in the Asia-Pacific region (top), and the change in percent tree cover on agricultural land from the year 2000 to 2010 (bottom).

Figure 3. Percent tree cover on agricultural land in the Asia-Pacific region in the years 2000 and 2010

Figure 4. Tree cover on agricultural land in 2010 in the Asia-Pacific region, showing cumulative area in $\mathrm{km}^{2}$, and cumulative area as a percent of total agricultural area for the region

Figure 5. The areal extent of tree-cover classes (10-20\%; 20-30\%; >30\%) on agricultural land in the Asia-Pacific region in the years 2000 and 2010, averaged by country $\left(\mathrm{km}^{2}\right)$

Figure 6. The relative areal extent of tree-cover classes (10-20\%; 20-30\%; >30\%) on agricultural land in the Asia-Pacific region in the years 2000 and 2010, averaged by country, shown as a percent of the total agricultural land within that country (\%)...

Figure 7. East Asia: Percent tree cover on agricultural land in the year 2010, and the change in percent tree cover on agricultural land from the year 2000 to 2010

Figure 8. Indo-Pacific: Percent tree cover on agricultural land in the year 2010, and the change in percent tree cover on agricultural land from the year 2000 to 2010

Figure 9. Southeast Asia: Percent tree cover on agricultural land in the year 2010, and the change in percent tree cover on agricultural land from the year 2000 to 2010

Figure 10. South Asia: Percent tree cover on agricultural land in the year 2010, and the change in percent tree cover on agricultural land from the year 2000 to 2010

\section{List of tables}

Table 1. Percent tree cover on agricultural land in the Asia-Pacific region in the years 2000 and 2010, averaged by country, and the decadal change in percent tree cover

\section{List of photographs}

Photo 1. Mixed tree system, Vientiane, Lao PDR. Photo: World Agroforestry/Robert Finlayson.

Photo 2. Researcher Dede Rohadi of the Center for International Forestry Research holds fruit of the jengkol tree, Archidendron pauciflorum, intercropped with oil palm, Elaeis guineensis, in Pontianak, Indonesia. Photo: World Agroforestry/Center for International Forestry Research/Robert Finlayson 
Photo 3. Early stage of a 50-ha agroforestry landscape in Yen Bai Province, Viet Nam. Photo: World Agroforestry/Yudi Nofiandi

Photo 4. Re-agroforested landscape in Mindanao, The Philippines. Photo: World Agroforestry/Robert Finlayson

Photo 5. Farmers planning agroforestry in DPR Korea. Photo: World Agroforestry .13

Photo 6. Silvopastoral agroforestry practices with trees and livestock. Timor, Kupang, Indonesia. Photo: World Agroforestry/Ingrid Öborn...

Photo 7. A paddy-based agroforestry system in Sri Lanka. Photo: World Agroforestry/DKNG Pushpakumara

Photo 8. Tree in field integrated agroforestry system with animal husbandry, Nepal. Photo: World Agroforestry

Photo 9. Poplar-wheat intercrop systems on irrigated farmland are found extensively across the northern parts of Haryana and Uttar Pradesh States, India. Photo: World Agroforestry/Robert Zomer. 


\section{About the authors}

Robert J. Zomer is a senior research scientist, landscape and systems ecologist, with broad background in plant community, forest and agricultural ecology, using statistical analysis, geographic information systems, remote sensing, environmental modelling, and landscape-level spatial analysis for sustainable development and biodiversity conservation. Special focal areas includes modelling impacts of climate change on terrestrial ecosystems, agroforestry, agro-ecosystems, protected areas, and mountain landscapes. He has had extensive working experience as a research scientist and/or program leader in various projects and at various research institutes in Africa and Asia, including World Agroforestry (ICRAF) in Nairobi; International Water Management Institute in Sri Lanka; and the International Center for Integrated Mountain Development in Kathmandu. He is currently Visiting Professor at the Centre for Mountain Ecosystem Studies, Kunming Institute of Botany, Chinese Academy of Sciences in Kunming, China.

Ingrid Öborn is a Senior Research Fellow with World Agroforestry and Professor of Agricultural Cropping Systems at the Swedish University of Agricultural Sciences. She holds a PhD in Soil Science. Her research interests include the multi-functionality of agro-ecosystems, including agroforestry, integrating improved productivity and natural resource management with food and nutrition security, and their synergies and trade-offs; biogeochemistry: nutrient and trace element flows and dynamics in agricultural systems and the food chain, including soil mineral weathering; and foresight and future studies and methodologies related to food security, agricultural systems and natural resource management. She was Regional Coordinator, World Agroforestry Southeast Asia Regional Program during 3-years 2016-2018.

Jianchu Xu is Principal Scientist and Regional Coordinator, World Agroforestry East and Central Asia Regional Program; Distinguished Professor, Kunming Institute of Botany, Chinese Academy of Sciences; and Distinguished Professor, Institute of Animal Sciences, Chinese Academy of Agricultural Sciences. He is an ethnobotanist working on land systems in coupled social-ecological systems. He has a strong background in interdisciplinary research, with a ranking in the top $1 \%$ of scientists globally for the most-cited documents in plant and animal sciences in 2017 and cross-field in 2018. His current research includes investigation of early warning signals of climate change, ecosystem restoration, forest management, fungi ecology, and integrative tree-crop-livestock systems. After more than 30 years of living and working in the eastern Himalayas, he has excellent scientific research networks in Asia. Xu has published over 300 SCI journal articles including in Nature, Science, Proceedings of the National Academy of Sciences, Fungal Diversity, Frontiers in Ecology and Evolution, Global Environmental Change, PLOS Biology and Conservation Biology. He is coordinating the Mountain Futures Program, which is a global initiative to support knowledge innovations for smallholders in mountain regions worldwide. 


\begin{abstract}
The study provides a geospatial analysis of tree cover on agricultural land within the Asia-Pacific region. It gives statistics at the national and provincial levels for the year 2010, and change since the year 2000, as well as a spatial mapping of tree cover and change. For the Asia-Pacific region, the average percent tree cover on agricultural land was 15\% in 2000 and $16.3 \%$ in 2010. In India and China, having the largest areas of agricultural land in the region followed by Indonesia, it was 7.8 and $12.8 \%$, respectively, while in Indonesia it was $43.1 \%$. The regional variation broadly follows climatic zones, with high tree cover in the more humid regions, such as Southeast Asia and the Pacific, and less tree cover in drier areas in South Asia and western China. Tree cover on agricultural land has decreased 1.2-2.2\% during one decade (2000-2010) in Cambodia, Lao PDR, Japan and Myanmar. The highest increase in tree cover (3.2-5.7\%) was observed in Timor-Leste, Bangladesh, Bhutan, Indonesia, Malaysia, Vanuatu, New Zealand and Fiji. In India, China, Papua New Guinea, Philippines and Thailand, tree cover increased 1.0-2.4\% from 2000 to 2010. More than $44 \%$ of the 6.7 million $\mathrm{km}^{2}$ of agricultural land within the region had more than $10 \%$ tree cover in 2010 , comprising nearly 2.7 million $\mathrm{km}^{2}$. This is a $4.3 \%$ larger area than in 2000 . Over $24 \%$ of agricultural land has greater than $20 \%$ tree cover and $16 \%$ of the area has more than $30 \%$ tree cover. This highlights the important roles of tree cover on agricultural land across the region. The least areas of tree cover were found in Afghanistan, Pakistan and Mongolia, with only 1-8\% of agricultural land having greater than $10 \%$ tree cover in 2010. The highest proportion of agricultural land with tree cover, of 10-20\%, was found in Bangladesh (60\%) followed by Sri Lanka (31\%) and Vietnam, China, Nepal, Thailand and DPR Korea (24-28\%). In New Zealand, 34\% of the agricultural area had 20-30\% tree cover and 22-27\% in Fiji, Nepal, Sri Lanka, Timor-Leste and The Philippines. Tree cover greater than $30 \%$ was found in more than half of the area of agricultural land in ten countries; Vanuatu, Bhutan, Malaysia, Papua New Guinea, Lao PDR, Indonesia, Japan, Fiji and Timor-Leste. Recognizing the extension of trees on farms and in agricultural landscapes is crucial as environmental and climatic change in the Asia Pacific region is rapidly accelerating. The role of trees for rural livelihoods, sustainability and resilience of agricultural landscapes, and for climate-change mitigation, has brought the need for agroforestry practices and supporting policies and institutions to the forefront of sustainable development. The report shows that efforts during the last decades have had some success but that there is large potential for increasing tree cover within many of the countries of the region.
\end{abstract}

\title{
Keywords
}

Tree cover, Asia-Pacific, agriculture, land 


\section{Acknowledgements}

The authors would like to acknowledge the initial support for this work and previous global tree-cover analysis completed at the Centre for Mountain Ecosystem Studies, a joint laboratory of World Agroforestry East and Central Asia Region and the Kunming Institute of Botany, Chinese Academy of Sciences. This previous research was partly supported by the National Key Basic Research Program of China (Grant No. 2014CB954100) and National Science Foundation China (Grant No. 31270524). Additional support was also provided from the CGIAR research programs on Forests, Trees and Agroforestry; and Climate Change, Agriculture and Food Security. Support was also provided by the Asia-Pacific Association of Forestry Research Institutions and the Food and Agriculture Organization of the United Nations Asia-Pacific Region (FAO-RAP) as part of the AsiaPacific Forestry Sector Outlook Study III (LoA/RAP?2017/41).

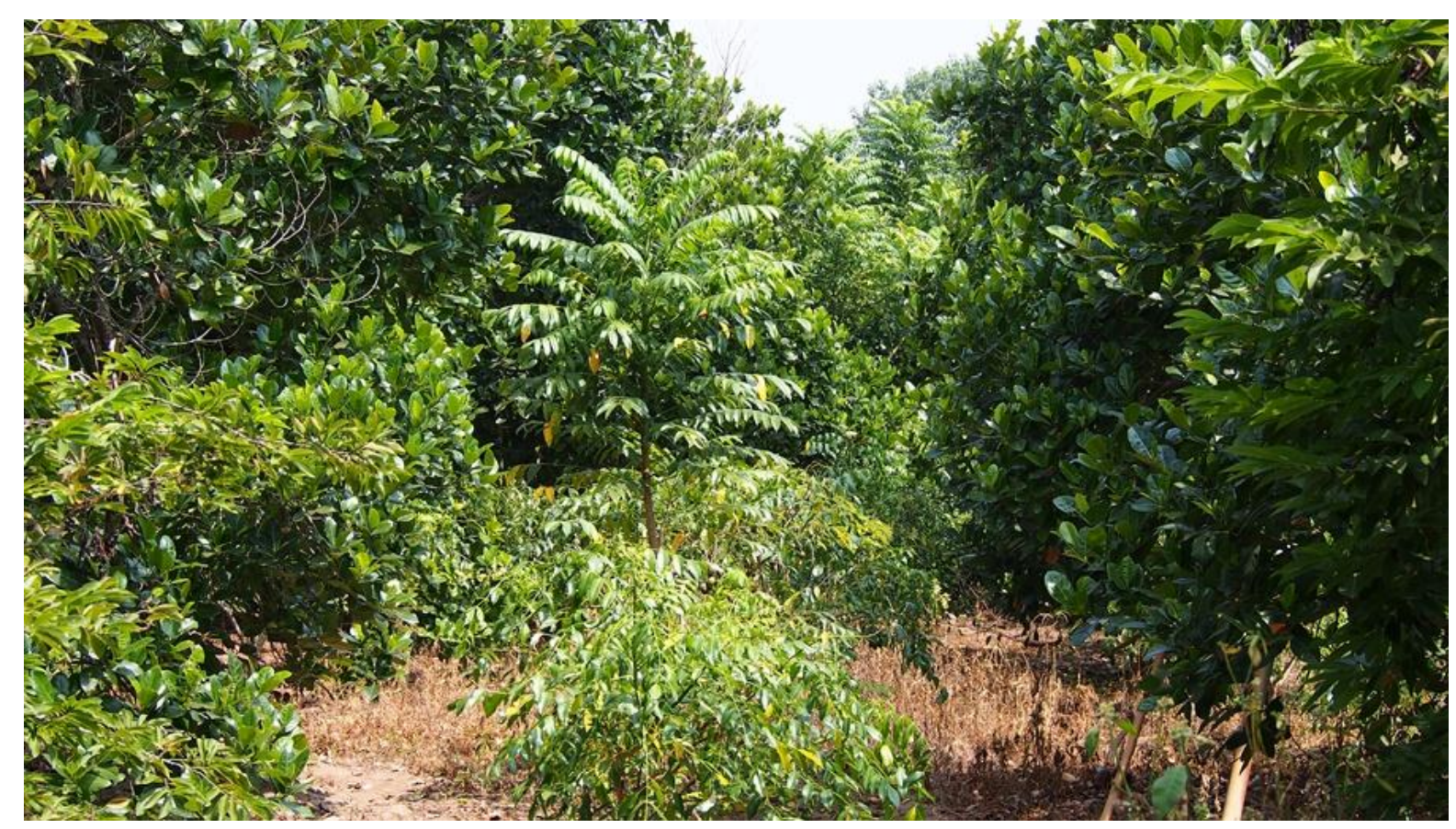

Photo 1. Mixed tree system, Vientiane, Lao PDR. Photo: World Agroforestry/Robert Finlayson 


\section{Introduction}

Trees outside forests comprise tree formations ranging from single, discrete trees to systematically managed trees in agroforestry systems. Within agricultural systems and landscapes, they serve a number of ecological and economic functions which may be similar to those of forests in principle although different in extent. TOF have been little recognized in natural resources assessments, particularly for large areas, and it is only recently that this topic has emerged as a significant research issue. Zomer et al (2009) first attempted to quantify the global extent and geographical patterns of agroforestry, using tree cover on agricultural land, based upon then recently released remote sensing datasets. Zomer et al (2014) updated and reanalyzed the analysis of global extent and socio-ecological characteristics, based upon improved higher quality data at $250 \mathrm{~m}$ resolution. In addition, Zomer et al (2014) was able to provide a decadal trend analysis, that is, identifying change in tree cover from 2000 to 2010.

The goal and purpose of this study were to provide regional and national-level analyses of tree cover on agricultural land (using Zomer et al 2014) for the Asia-Pacific region. The study is a contribution to the Food and Agriculture Organization of the United Nation's (FAO) Asia-Pacific Forestry Outlook Study.

The scope of this analysis includes both regional- and national-level analyses. Thirty-four countries were initially analyzed, however, only 27 are included in the national-level analysis owing to data constraints and/or lack of a significant amount of agricultural area.

\section{Regional analysis}

1) A geospatial analysis of tree cover in the years 2000 and 2010 and change from 2000, was tabulated for the region.

2) Geospatial mapping of distribution of tree cover on agricultural land in 2010 and change since 2000.

\section{National analysis}

1) Tree cover in 2010 and change from 2000 was tabulated for each country by sub-national (for example, provincial) administrative units (GADM - 2018 - Level 1) and provided in summary tables

2) A set of national-level maps, showing sub-national administrative boundaries, have been produced for each country, showing tree cover in 2010, and change since 2000.

3) An appendix is provided as supplementary material:

a. A set of national-level tables for each country

b. A set of high-resolution maps for each country showing tree cover and change

\section{Background}

The inclusion of trees within farming systems, which can also be interpreted as agroforestry, is and has been a traditional land use developed by subsistence farmers throughout almost all of the regions of the world. This is particularly true for the Asia-Pacific.

Over the last 40 years, agroforestry has become a subject for systematic study and improvement. It is a livelihoods' option often mentioned and increasingly promoted by land-use managers and international development agencies. More recently, agroforestry has come to the attention of global 
analysts and national policy makers concerned with environmental sustainability, and both climatechange adaptation and mitigation.

However, trees on agricultural land, similar to 'trees outside of forests' in general, have traditionally been neglected by national and global forest surveys and, similarly, are difficult to discern and measure. Tree cover on agricultural land, while playing important roles both biophysically and economically, is generally not adequately reflected within many national programs. This includes countries where it may play a very important economic role, especially at the household level.

Similar to the 'trees outside forests' concept, as defined by the Food and Agriculture Organization of the United Nations (FAO) (de Foresta et al 2013), an increased understanding and better estimates for the extent and role of trees on agricultural land can provide the knowledge base for improved policies and enhanced policy support.

Zomer et al $(2009,2014,2016)$ was a first attempt to quantify the extent of agroforestry at the global level (Figure 1). Several surprising results were reported, not the least of which was the unexpectedly high level of the extent of agroforestry. Approximately $43 \%$ of all agricultural land had at least $10 \%$ tree cover. Notably, this corresponds with several definitions of 'forest', including as one parameter for the current FAO definition. Among the key results of looking at trees on agricultural land were that agroforestry is a significant feature of agriculture in all regions of the world; that its extent varies significantly across different regions; that tree cover is strongly positively related to humidity; and that there are mixed relationships between tree cover and population density, depending on the region.

The countries of the Asia-Pacific region included in this study span a wide geographic expanse, an extremely diverse range of bioclimatic, environmental, bio-physical and climatic conditions, and are extremely culturally diverse. Each has some history or tradition of indigenous agroforestry as well as development programs or projects promoting adoption of agroforestry practices.

However, the Asia-Pacific has undergone, and is under-going, rapid socio-economic, environmental and landscape change, and this is reflected in the change in tree cover on agricultural land. Tree cover generally decreases with increased intensification, however, much agricultural land in the region is also being converted to tree plantations or being reforested.

In this study, we provide an analysis of tree cover at the national level for countries within the AsiaPacific region, which both give statistics at the national and provincial levels for the year 2010, and change since the year 2000, as well as a spatial mapping of tree cover and change. Details of the data sources and methodology are given in the Methods section and further described in Zomer et al (2009, 2014). 


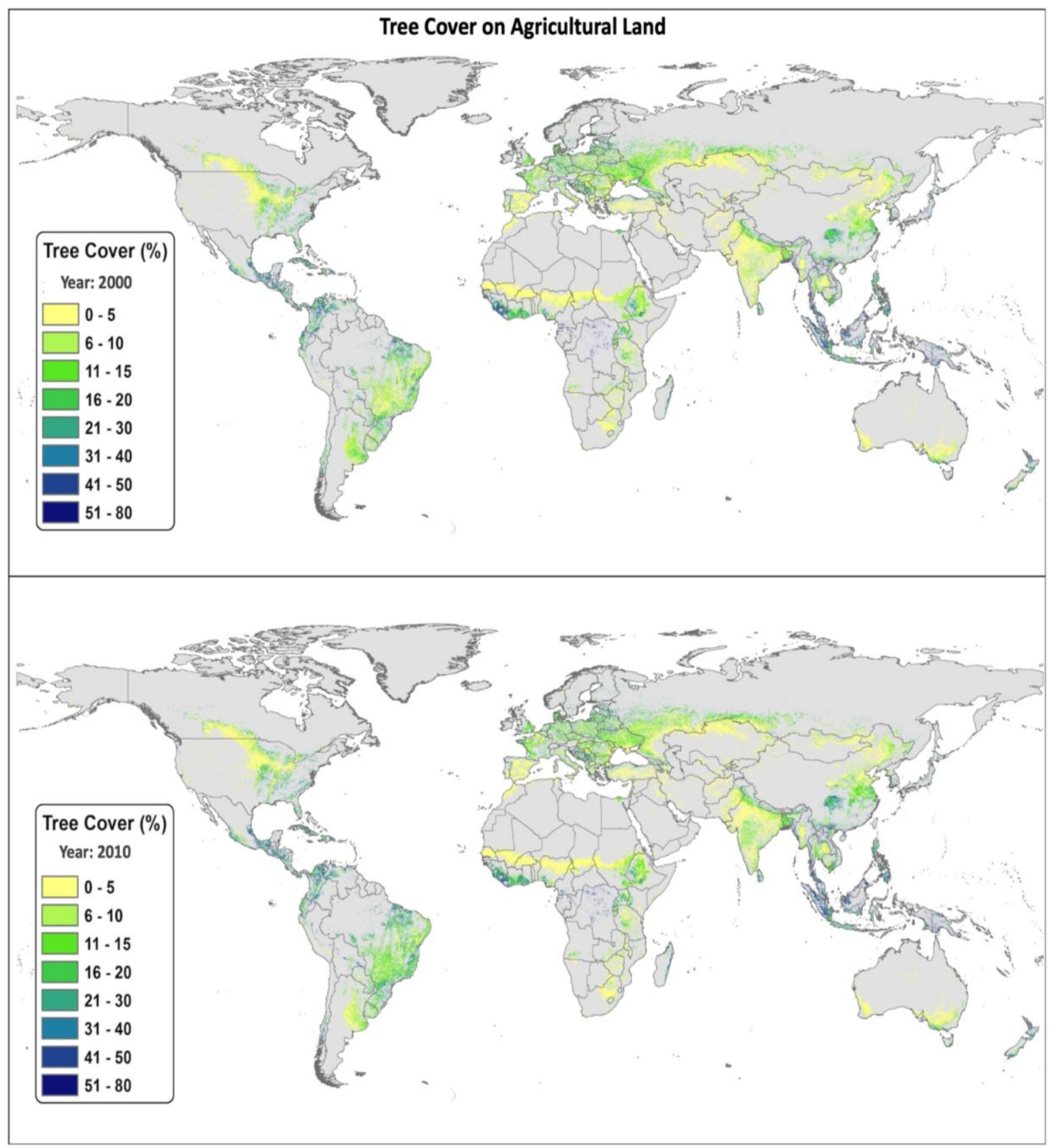

Figure 1. Tree cover on agricultural land, globally, 2000 and 2010 


\section{Methods}

The global geospatial analysis in Zomer et al $(2014,2016)$ combined tree cover with a land-use classification to identify agricultural land.

In this current analysis, we have used the results from that analysis to delineate and tabulate tree cover within national and sub-national (provincial) boundaries of 26 countries within the Asia-Pacific region.

The spatial modelling procedure was developed and implemented in ArcGIS Pro 2.2 (ESRI Inc.) using the Python programming language. The datasets used for the analyses were re-projected into the Sphere Sinusoidal coordinate system (ESRI: 53008). This projection maintains equal area despite conformal distortion, allowing for accurate measurement of areal extent. The sinusoidal projection has been used to calculate zonal statistics and carry out areal computations as it represents area extent accurately across latitudes (that is, equal-area projection). The cell size for analyses in sinusoidal projection is $1 \mathrm{~km}^{2}$.

The tree-cover dataset produced in the Zomer et al (2014) analysis was based upon an analysis of MODIS $250 \mathrm{~m}$ resolution remote-sensing data produced by the University of Maryland, with a global land-cover database produced by the United States Geological Survey:

- MOD44B MODIS Vegetation Continuous Field Coll. 5-2000 through to 2010: Percent Tree Cover (Di Miceli et al 2011)

- Global Land Cover 2000 Database (GLC 2000)

\section{Land-cover categories}

Three agricultural land-use types from the Global Land Cover Class scheme used for the Global Land Cover 2000 database were selected as relevant for the specific objectives of this work: Cultivated and managed areas (Agriculture - intensive), Cropland/Other natural vegetation (non-trees) (Mosaic agriculture/degraded vegetation) and Cropland/Tree Cover Mosaic (agriculture/degraded forest).

The mix of tree cover over agricultural land is depicted along a continuous gradient by the MODIS VCF tree-cover dataset, within the relevant GLC2000 land-cover type. Tree cover shows the percentage of the $1 \mathrm{~km}^{2}$ grid cell occupied by trees, therefore, at this resolution of $1000 \mathrm{~m}$, the treecover percentage can be expressed as hectares (ha) of tree cover per $\mathrm{km}^{2}$. At $100 \%$ tree cover, the whole grid cell is occupied, that is, $100 \mathrm{ha} / \mathrm{km}^{2}$.

\section{Tree-cover data}

The MOD44B MODIS/Terra Vegetation Continuous Fields Dataset (VCF) - Collection 5 dataset used in the Zomer et al (2014) analysis provides data at a relatively high resolution of 250 metre for the entire globe. The 250-metre resolution grid cells were aggregated to $1 \mathrm{~km}$ resolution. Datasets from 2000-2002 and 2008-2010 were averaged to reduce inter-annual variability and are herein referred to as the year 2000 and 2010, respectively. All the geo-datasets were masked to exclude areas which are either non-agricultural land-use types or urban areas.

For the Pacific, there was no available tree-cover data for the countries of Kiribati, Maldives, Samoa, Tonga and Tuvalu, where tree-data pixels were lacking. Samoa was unfortunately missing in the original global study (Zomer et al 2009, 2014) and could not be added at this stage. The other countries, all of which are small island states, have very little agricultural land (or at least agricultural 
land that would be seen in the remote-sensing analysis) and were not included in the agricultural land database we used in the analysis.

\section{Average Tree Cover Percent}

Tree cover within the analysis is provided as the percent of a $1 \mathrm{~km}^{2}$ grid cell of agricultural land covered by trees, ranging $0-86 \%$ for the Asia-Pacific region. As a rough estimate of the relative tree cover between regions, countries and sub-national units, the percent tree cover of all agricultural land has been averaged.

For the Asia-Pacific region as a whole (Figure 2), which has nearly 6.7 million $\mathrm{km}^{2}$ of agricultural land, the average percent tree cover was nearly $15 \%$ in the year 2000, and more than $16.3 \%$ in 2010 (Table 1). By comparison, the global average was $13.7 \%$ in 2000, increasing to 14.4 by 2010 (Figure 2). As in the global analysis, the regional variation broadly follows climatic zones, with high tree cover in the more humid regions, such as Southeast Asia and the Pacific, while drier regions in South Asia and western China have markedly less tree cover.

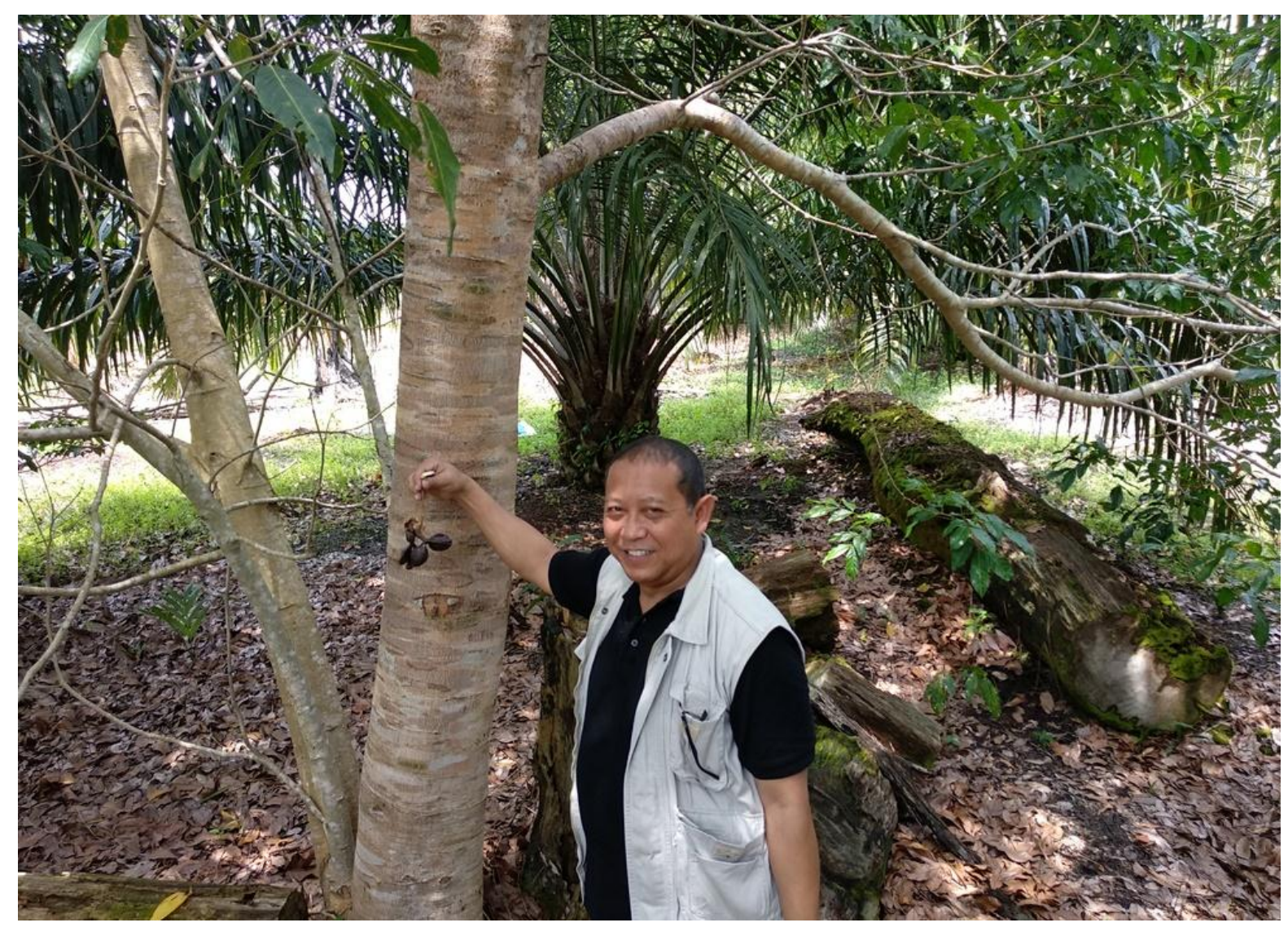

Photo 2. Researcher Dede Rohadi of the Center for International Forestry Research holds fruit of the jengkol tree, Archidendron pauciflorum, intercropped with oil palm, Elaeis guineensis, in Pontianak, Indonesia. Photo: World Agroforestry/Center for International Forestry Research/Robert Finlayson 
Table 1. Percent tree cover on agricultural land in the Asia-Pacific region in the years 2000 and 2010, averaged by country, and the decadal change in percent tree cover

\begin{tabular}{lrrrr} 
Country & $\begin{array}{c}\text { Agricultural area } \\
(\mathbf{k m 2})\end{array}$ & \multicolumn{3}{c}{ Mean tree cover percent by country $(\%)$} \\
\cline { 3 - 5 } Afghanistan & 118,964 & 2000 & 2010 & Change \\
\hline Australia & 597,873 & 0.45 & 0.58 & 0.13 \\
\hline Bangladesh & 101,441 & 12.16 & 5.29 & 0.68 \\
\hline Bhutan & 6,485 & 48.32 & 51.95 & 3.61 \\
\hline Cambodia & 55,811 & 15.35 & 14.21 & 3.63 \\
\hline China & $1,691,755$ & 11.36 & 12.84 & -1.15 \\
\hline Fiji & 2,786 & 34.67 & 40.42 & 1.48 \\
\hline India & $1,640,132$ & 6.84 & 7.82 & 5.74 \\
\hline Indonesia & 621,833 & 39.19 & 43.09 & 0.98 \\
\hline Japan & 48,010 & 43.76 & 42.07 & 3.89 \\
\hline Laos & 45,963 & 46.17 & 43.93 & -1.70 \\
\hline Malaysia & 106,780 & 46.76 & 51.37 & -2.24 \\
\hline Mongolia & 244,556 & 4.37 & 4.28 & 4.61 \\
\hline Myanmar & 192,963 & 19.86 & 17.67 & -0.09 \\
\hline Nepal & 49,339 & 18.60 & 18.74 & -2.19 \\
\hline New Zealand & 76,758 & 23.91 & 29.54 & 0.14 \\
\hline North Korea & 22,660 & 14.17 & 14.16 & 5.64 \\
\hline Pakistan & 273,250 & 3.92 & 4.62 & -0.01 \\
\hline Papua New Guinea & 103,070 & 49.85 & 52.29 & 0.70 \\
\hline Philippines & 188,841 & 33.79 & 35.58 & 2.44 \\
\hline South Korea & 30,833 & 28.20 & 28.64 & 1.80 \\
\hline Sri Lanka & 29,041 & 26.94 & 27.95 & 0.44 \\
\hline Thailand & 275,151 & 15.86 & 17.31 & 1.01 \\
\hline Timor-Leste & 6,411 & 30.49 & 33.66 & 1.45 \\
\hline Vanuatu & 6,984 & 63.99 & 3.17 \\
\hline Vietnam & 24.92 & 16.31 & 5.39 \\
\hline Asia Pacific & 15,934 & & 0.27 \\
\hline & & & 1.33 \\
\hline
\end{tabular}




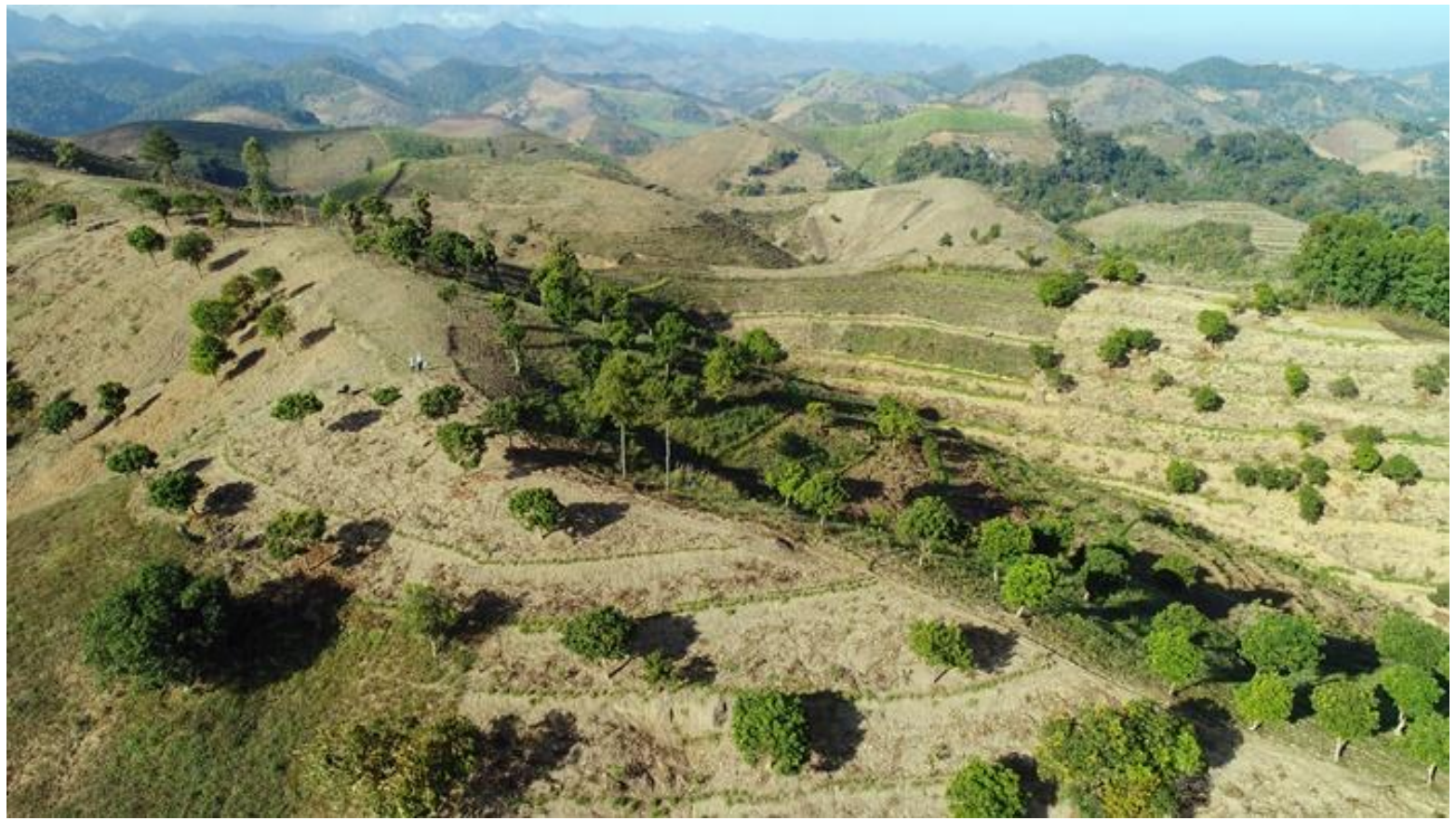

Photo 3. Early stage of a 50-ha agroforestry landscape in Yen Bai Province, Viet Nam. Photo: World Agroforestry/Yudi Nofiandi

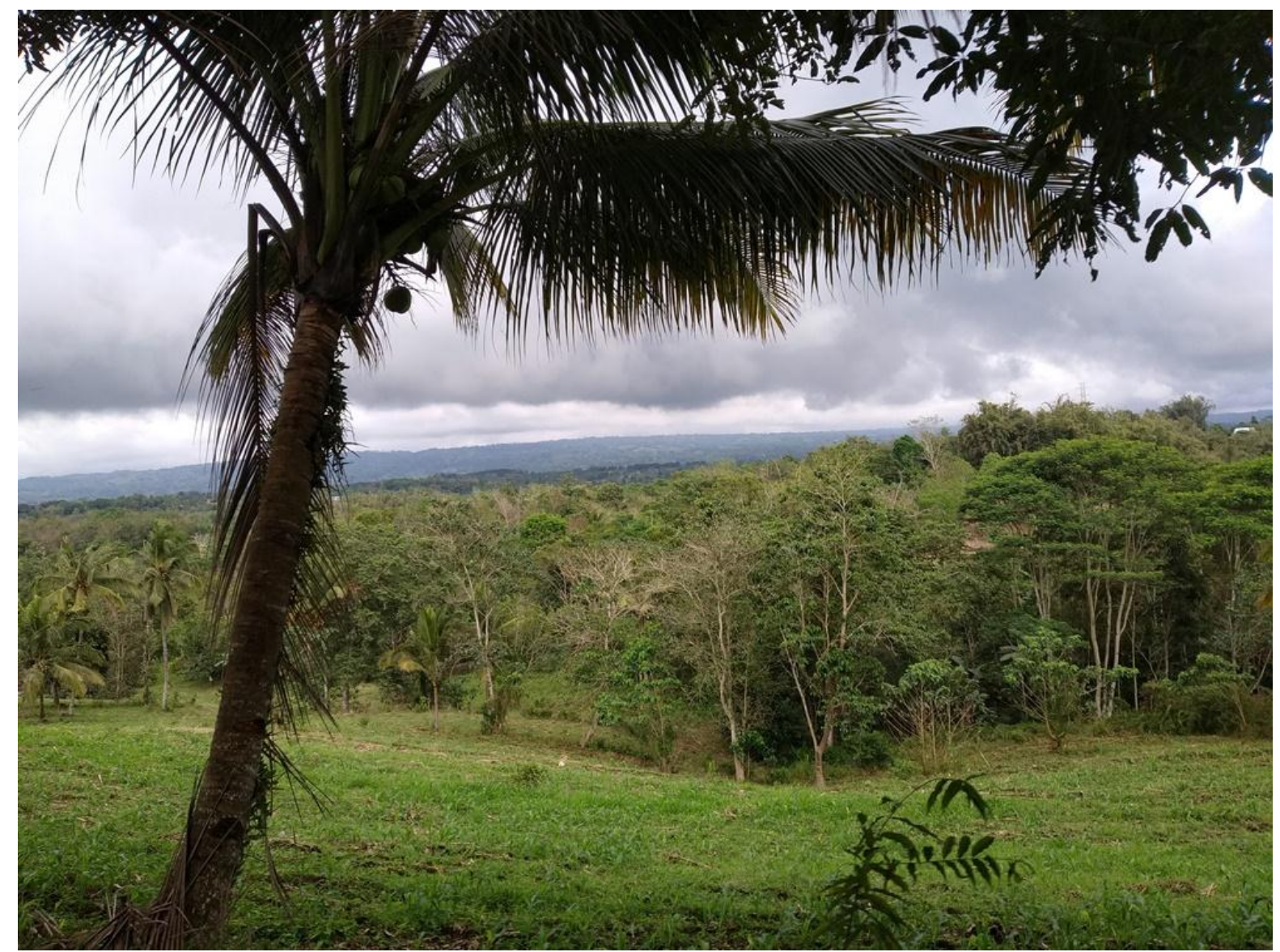

Photo 4. Re-agroforested landscape in Mindanao, The Philippines. Photo: World Agroforestry/Robert Finlayson 


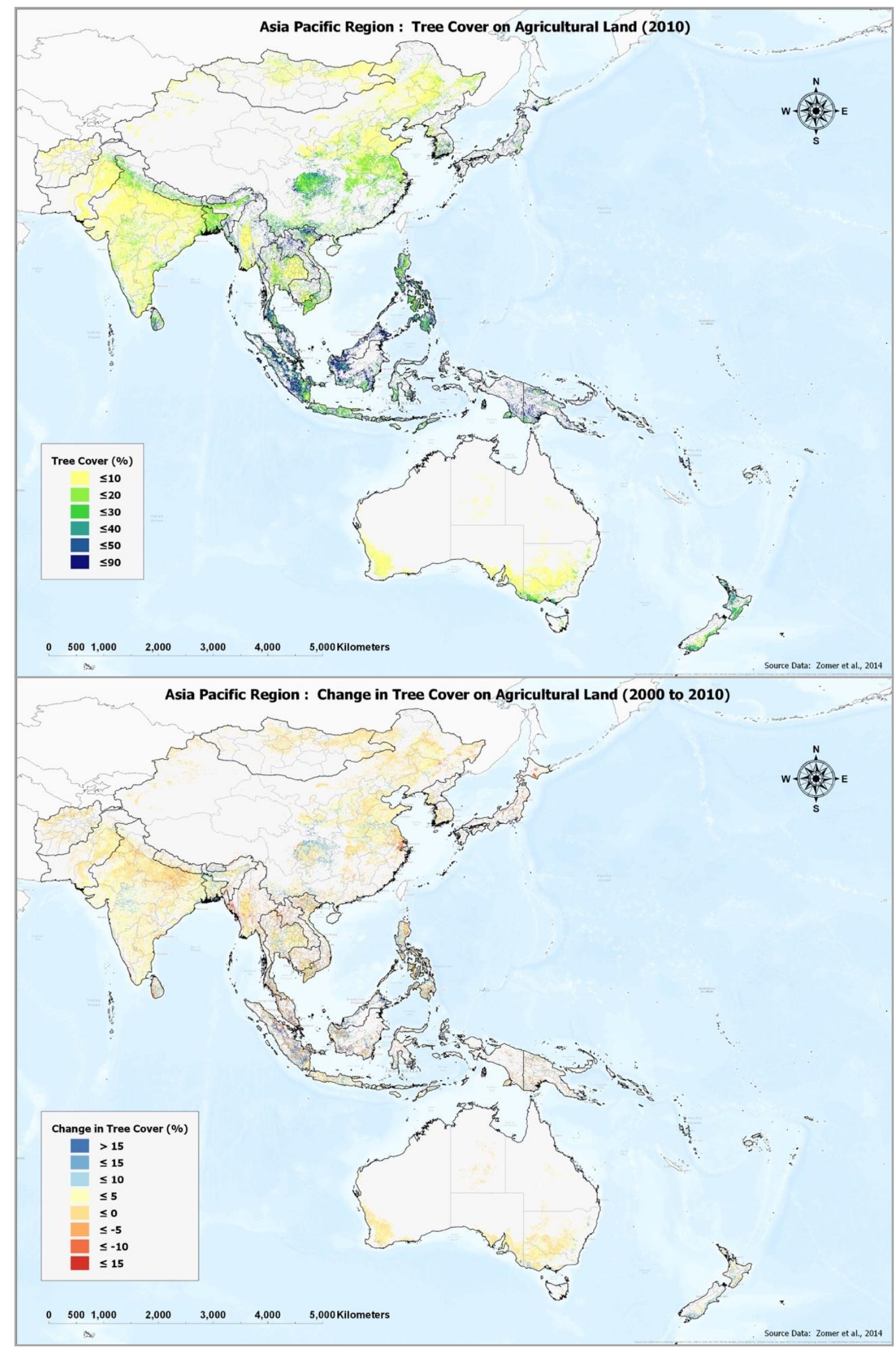

Figure 2. Percent tree cover on agricultural land in 2010 in the Asia-Pacific region (top), and the change in percent tree cover on agricultural land from the year 2000 to 2010 (bottom) 
Papua New Guinea, with over 100,000 $\mathrm{km}^{2}$ of agricultural land, had the highest mean tree-cover percent among countries in the region: over 50\%. However, China and India have the largest total amount of agricultural land in the region, each with more than 1.6 million $\mathrm{km}^{2}$, with China having an average of about $13 \%$ tree cover on agricultural land in 2010 and India less than $8 \%$. Indonesia, with more than $620,000 \mathrm{~km}^{2}$ of agricultural land, had a high average tree cover of nearly $44 \%$, which increased by $3.9 \%$ over the decade since the year 2000 . Vanuatu, with nearly $70 \%$ tree cover on agricultural land, but a relatively small amount of such land, had the highest tree cover in the region, reflecting the important role of local trees and traditional Pacific Islands agroforestry practices.

As in the global analysis, humidity was found to have a major influence on tree cover. In general, more arid countries have lower tree cover, both in natural systems and managed agricultural systems, although much land in these countries may be irrigated. For example, Afghanistan has the lowest tree cover in the region with a bare $0.6 \%$ on $119,000 \mathrm{~km}^{2}$ of agricultural land. Likewise, Pakistan has less than $5 \%$ on over $273,000 \mathrm{~km}^{2}$, while Mongolia, a grassland country not known for its tree cover, has just under $4.6 \%$. By comparison, Australia, with nearly $600,000 \mathrm{~km}^{2}$ of agricultural land, had a similar $4.6 \%$ in the year 2000, which increased $0.7 \%$ over the decade to $5.3 \%$ in 2010 .

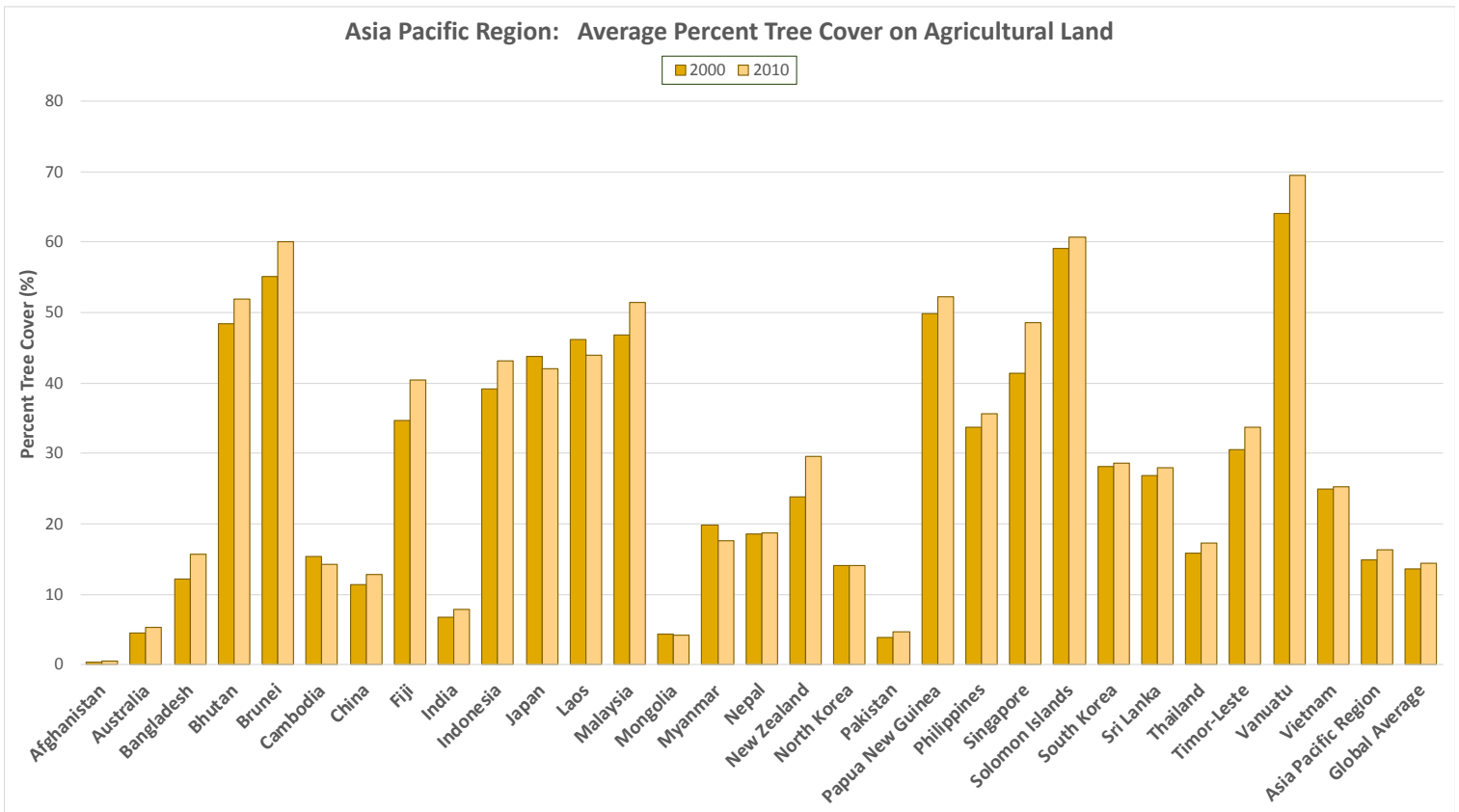

Figure 3. Percent tree cover on agricultural land in the Asia-Pacific region in the years 2000 and 2010, averaged by country

Overall, the Asia-Pacific region increased average percent tree cover by over $1.3 \%$ (Figure 3). Lao PDR and Myanmar showed the largest decrease in average percent tree cover, each with more than a $2 \%$ decrease, while Japan had a decrease of $1.7 \%$ and Cambodia just over 1.1\%. At least 13 countries in the region increased their average percent tree cover on agricultural land by more than $1 \%$, with eight of those countries increasing tree cover by more than $3 \%$. New Zealand, with over $76,000 \mathrm{~km}^{2}$ of agricultural land, increased average tree cover on that land by a remarkable $5.6 \%$ over the decade.

Malaysia, with over $106,0000 \mathrm{~km}^{2}$, increased it by $4.6 \%$, while Bangladesh with $101,000 \mathrm{~km}^{2}$ increased average tree cover by $3.6 \%$. In terms of number of trees planted, China's increase of nearly $1.5 \%$ on 1.6 million $\mathrm{km}^{2}$ represents a significant achievement, although its interpretation in terms of agroforestry is ambiguous, as much of this might be agricultural land being restored to native forest 
under the Grain for Green and similar initiatives. However, many of these lands have also been converted to fruit and nut orchards and other tree-farming practices.

\section{Areal Extent of Tree Cover}

More than $44 \%$ of the 6.7 million $\mathrm{km}^{2}$ of agricultural land within the Asia-Pacific region has more than $10 \%$ tree cover (Figure 4), comprising nearly 2.7 million $\mathrm{km}^{2}$. This reflects an increase in areal extent of $4.3 \%$ for land with more than $10 \%$ tree cover from 2000 to 2010, accounting for about $290,000 \mathrm{~km}^{2}$. Likewise, over $24 \%$ has greater than $20 \%$, and more than $16 \%$ has greater than $30 \%$, highlighting the important role of tree cover on agricultural land across the region (figures 5 and 6).

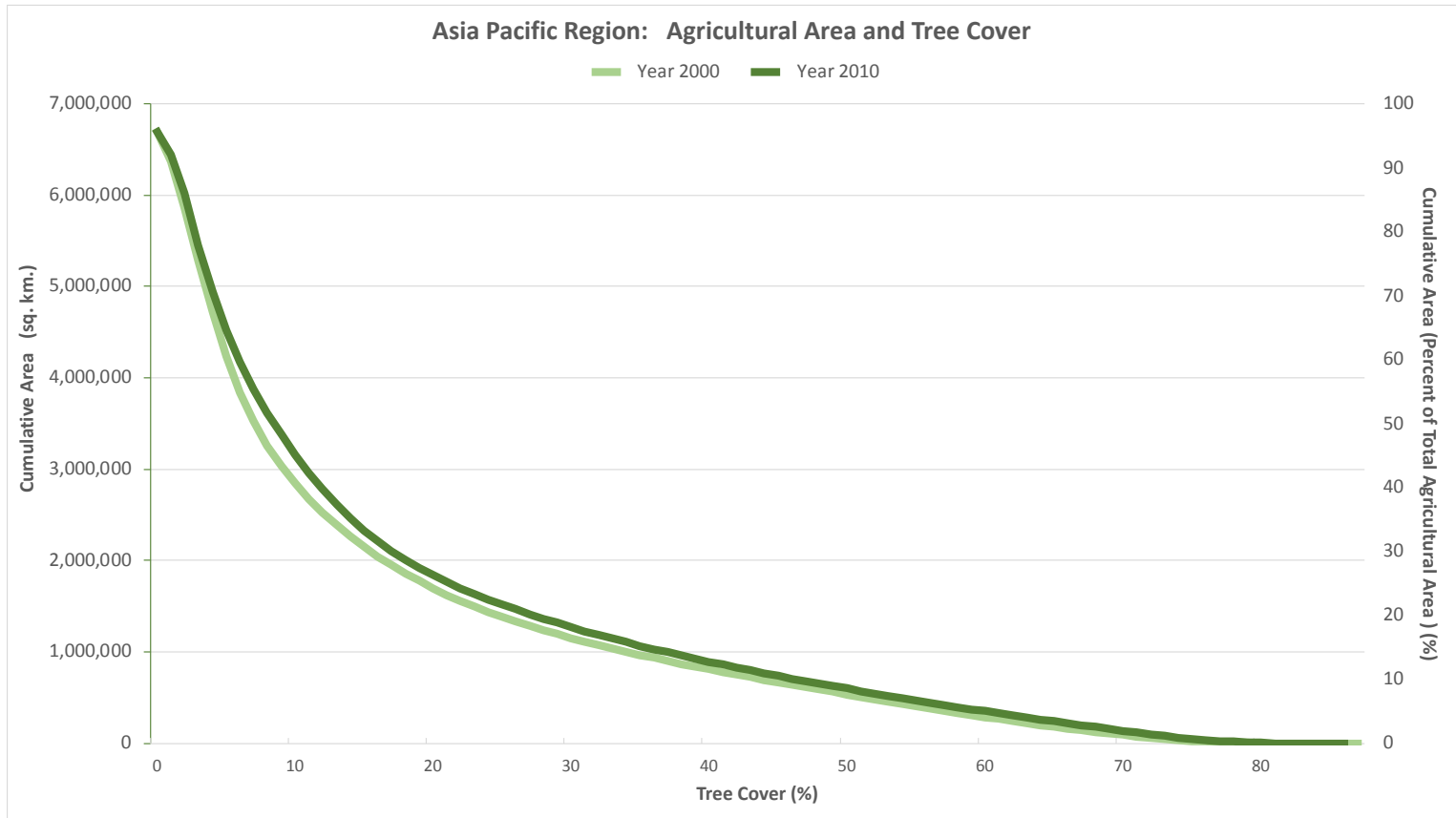

Figure 4. Tree cover on agricultural land in 2010 in the Asia-Pacific region, showing cumulative area in $\mathrm{km}^{2}$, and cumulative area as a percent of total agricultural area for the region

China had the greatest amount of agricultural land with more than $10 \%$ tree cover, almost 770,000 $\mathrm{km}^{2}$, comprising a full $45 \%$ of all the country's agricultural land and reflecting an increase of over $5 \%$ in the period 2000-2010. Indonesia had over $608,000 \mathrm{~km}^{2}$ of agricultural land with more than $10 \%$ tree cover, comprising an astonishing $98 \%$ of total agricultural land. Other countries with a large proportion of all agricultural land having at least $10 \%$ tree cover include Vanuatu (100\%), Fiji (100\%), Papua New Guinea (99\%), Malaysia (99\%), Philippines (98\%), Japan (97\%), Bhutan (96\%), Timor-Leste (94\%), New Zealand (94\%), Sri Lanka (92\%) and Lao PDR (91\%) (Figure 5).

In all, there are 16 countries above the global average of $43 \%$. Thailand was approximately near that mean in the year 2000 but increased over the decade by $9 \%$ so that now more than half of all agricultural land has more than $10 \%$ tree cover.

Cambodia, Myanmar and DPR Korea all have close to the global mean, however, Myanmar showed a slight decreasing trend over the decade whereas Cambodia showed a slight increasing trend. DPR Korea increased the amount of land with at least $10 \%$ tree cover by over $2.2 \%$. 


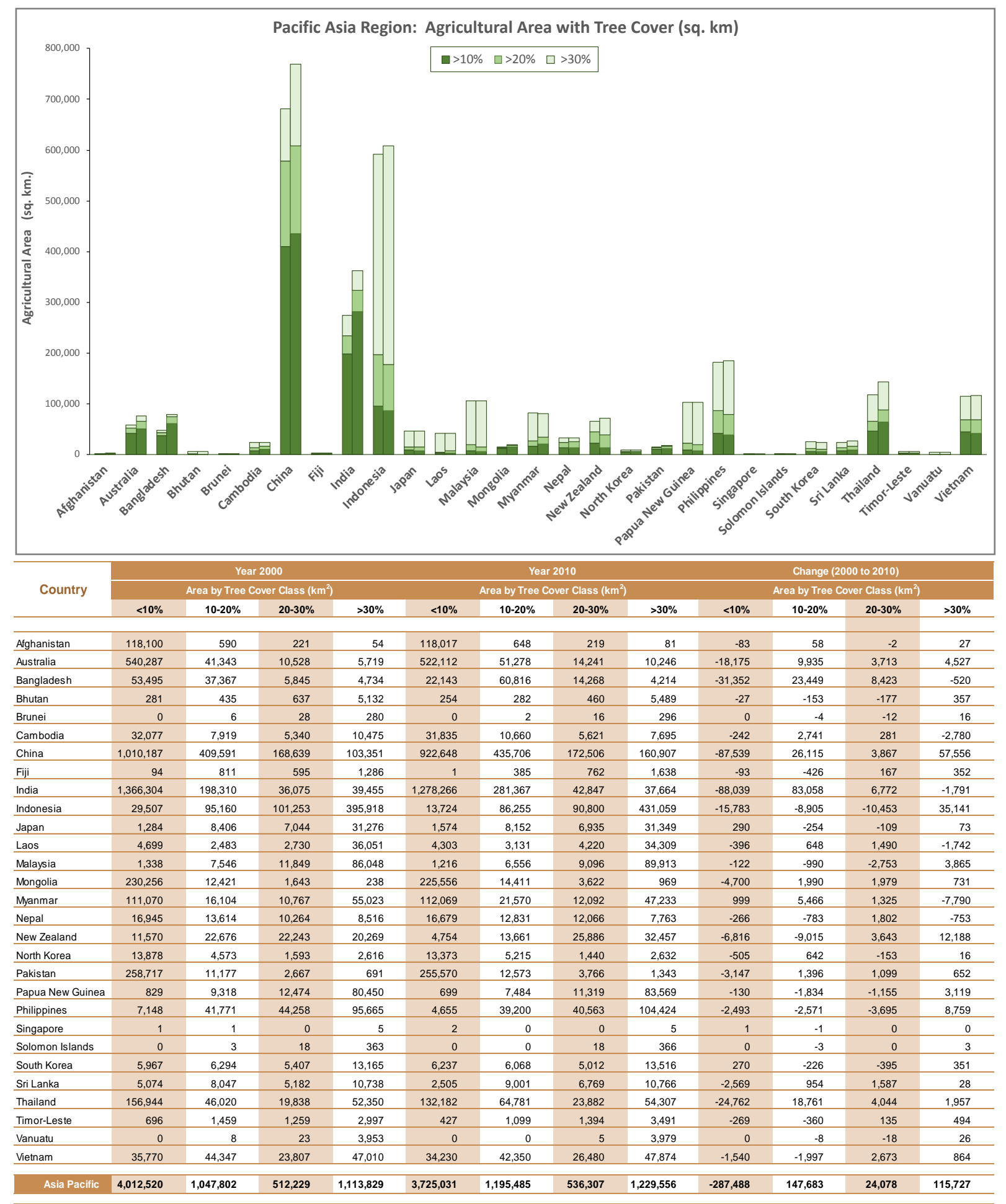

Figure 5. The areal extent of tree-cover classes (10-20\%; 20-30\%; >30\%) on agricultural land in the AsiaPacific region in the years 2000 and 2010 , averaged by country $\left(\mathrm{km}^{2}\right)$

Bangladesh was already above the mean in 2000, with over $47 \%$ of all agricultural land with greater than $19 \%$ tree cover, but then increased that amount by an astonishing $31 \%$ during the decade, to over $78 \%$.

By comparison, India has about $21.1 \%$ of its 1.6 million $\mathrm{km}^{2}$ of agricultural land having at least $10 \%$ tree cover but showed an increase of almost 5.4\% from 2000 to 2010 . Nevertheless, this still represents nearly $370,000 \mathrm{~km}^{2}$ of agricultural land. 
Likewise, Australia had just $12.7 \%$ of agricultural land with at least $10 \%$ tree cover, which represented an increase of just over 3\% from 2000. Mongolia, with 7.8\% in 2010, had increased by $1.9 \%$ from 2000, while Pakistan increased agricultural area with at least $10 \%$ tree cover from 5.3\% in 2000 to $6.5 \%$ in 2010 .

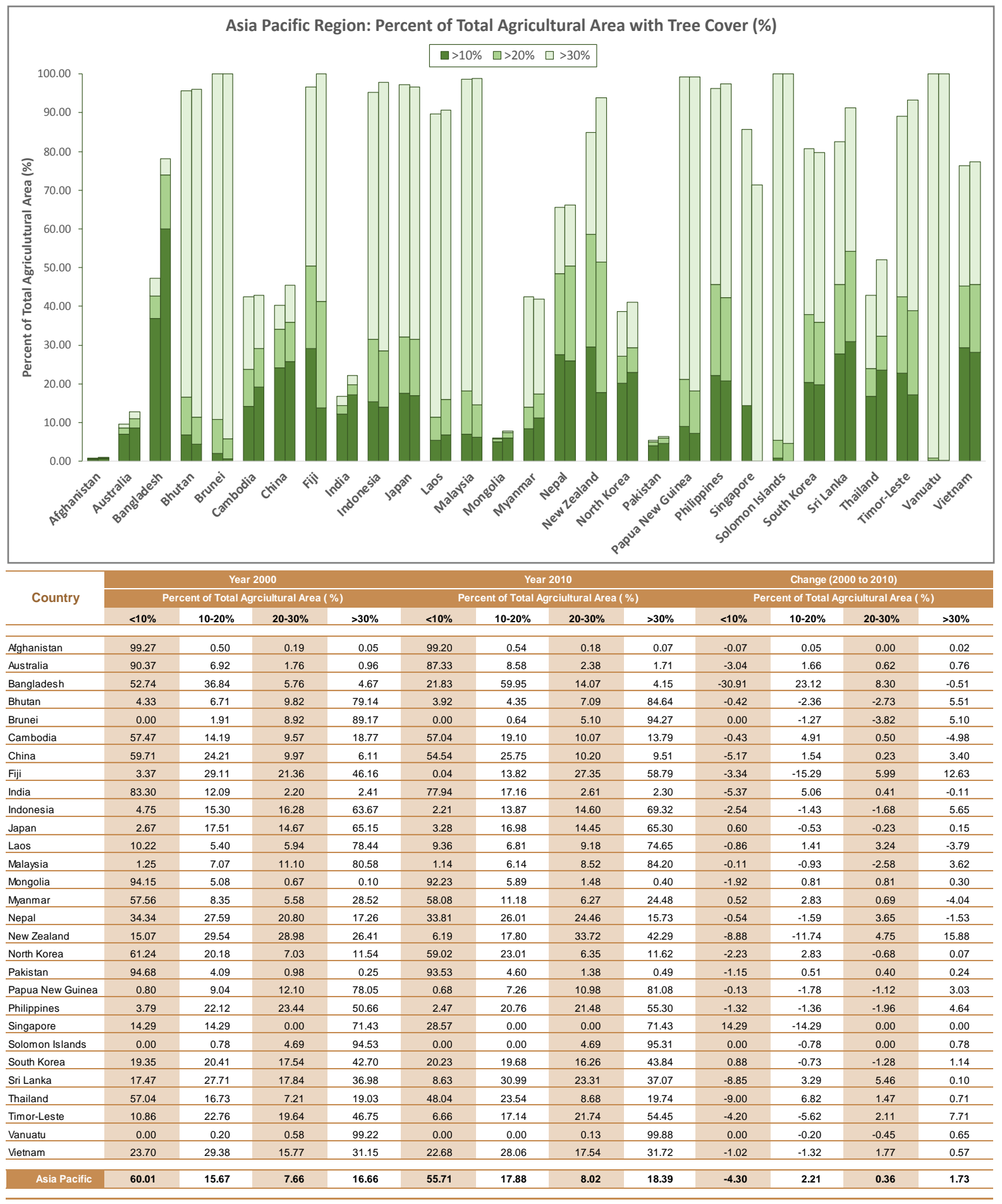

Figure 6. The relative areal extent of tree-cover classes (10-20\%; 20-30\%; >30\%) on agricultural land in the Asia-Pacific region in the years 2000 and 2010, averaged by country, shown as a percent of the total agricultural land within that country $(\%)$

Afghanistan had the least area of agricultural land with tree cover of at least $10 \%$, with less than $1 \%$ in 2000 , just barely increasing by $0.1 \%$ by 2010 . 
Bangladesh and Malaysia had the greatest area of land with tree cover of at least 30\%. Each had over $84 \%$, while Papua New Guinea also had over $80 \%$. However, Vanuatu had the highest proportion, with nearly $99 \%$ of all of its agricultural area supporting at least $30 \%$ tree cover. Afghanistan,

Pakistan and Mongolia each had less than $1 \%$ in this category.

These patterns are more fully illustrated in the regional maps below (Figures 7-10) and in the national maps and tables (articulated at the sub-national, provincial level) provided in the Country maps section.

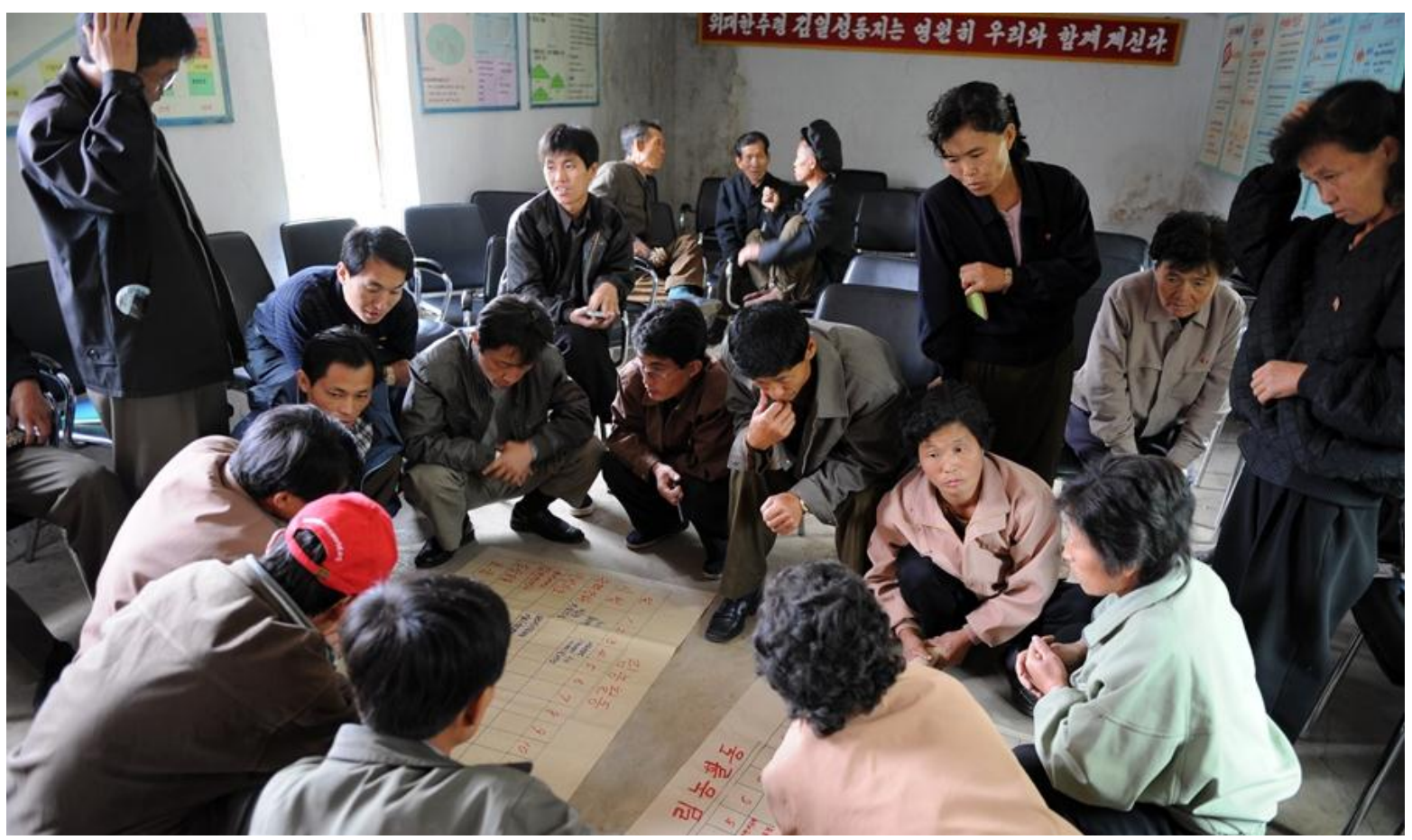

Photo 5. Farmers planning agroforestry in DPR Korea. Photo: World Agroforestry

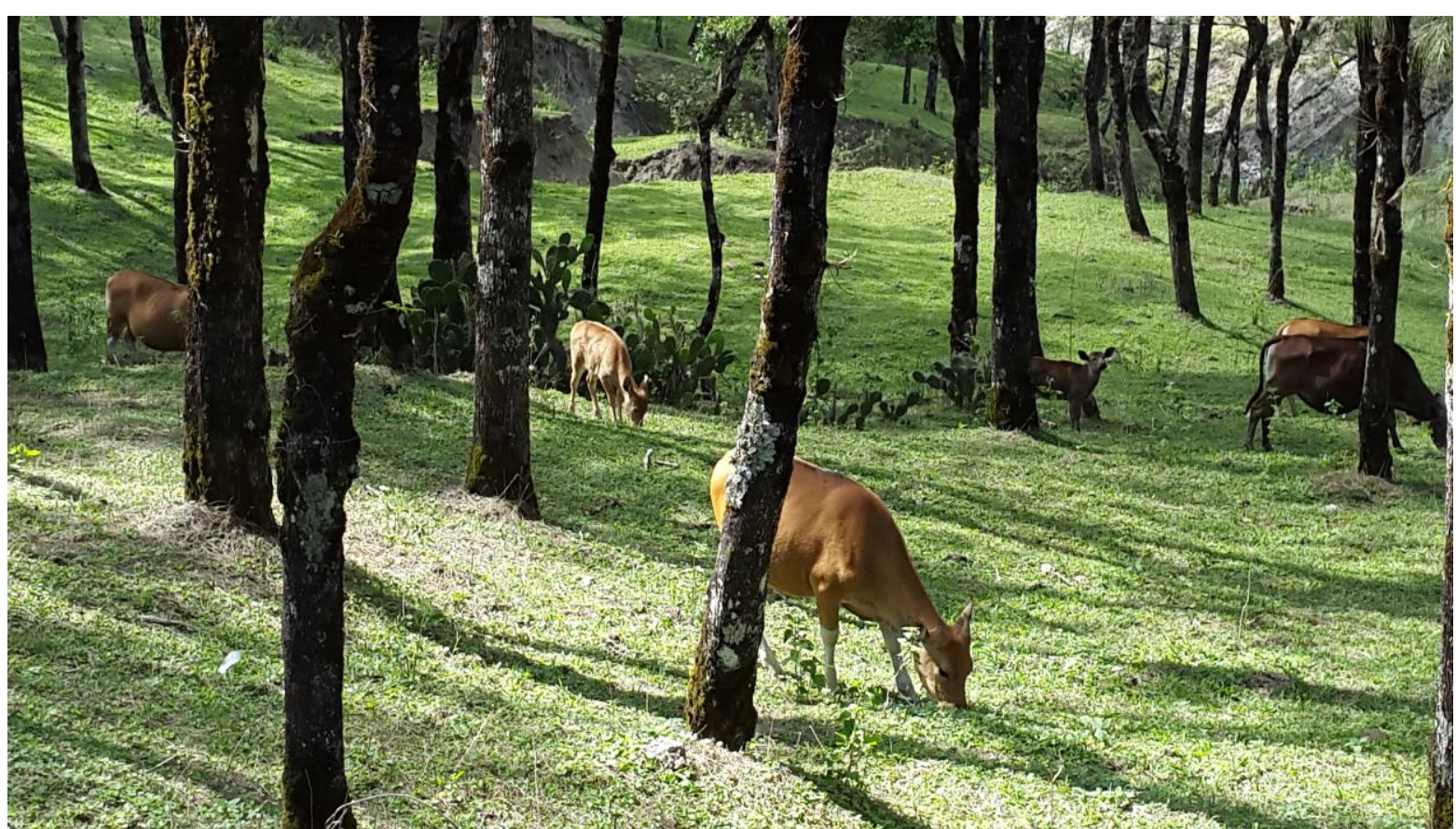

Photo 6. Silvopastoral agroforestry practices with trees and livestock. Timor, Kupang, Indonesia. Photo: World Agroforestry/Ingrid Öborn 


\section{Regional maps}
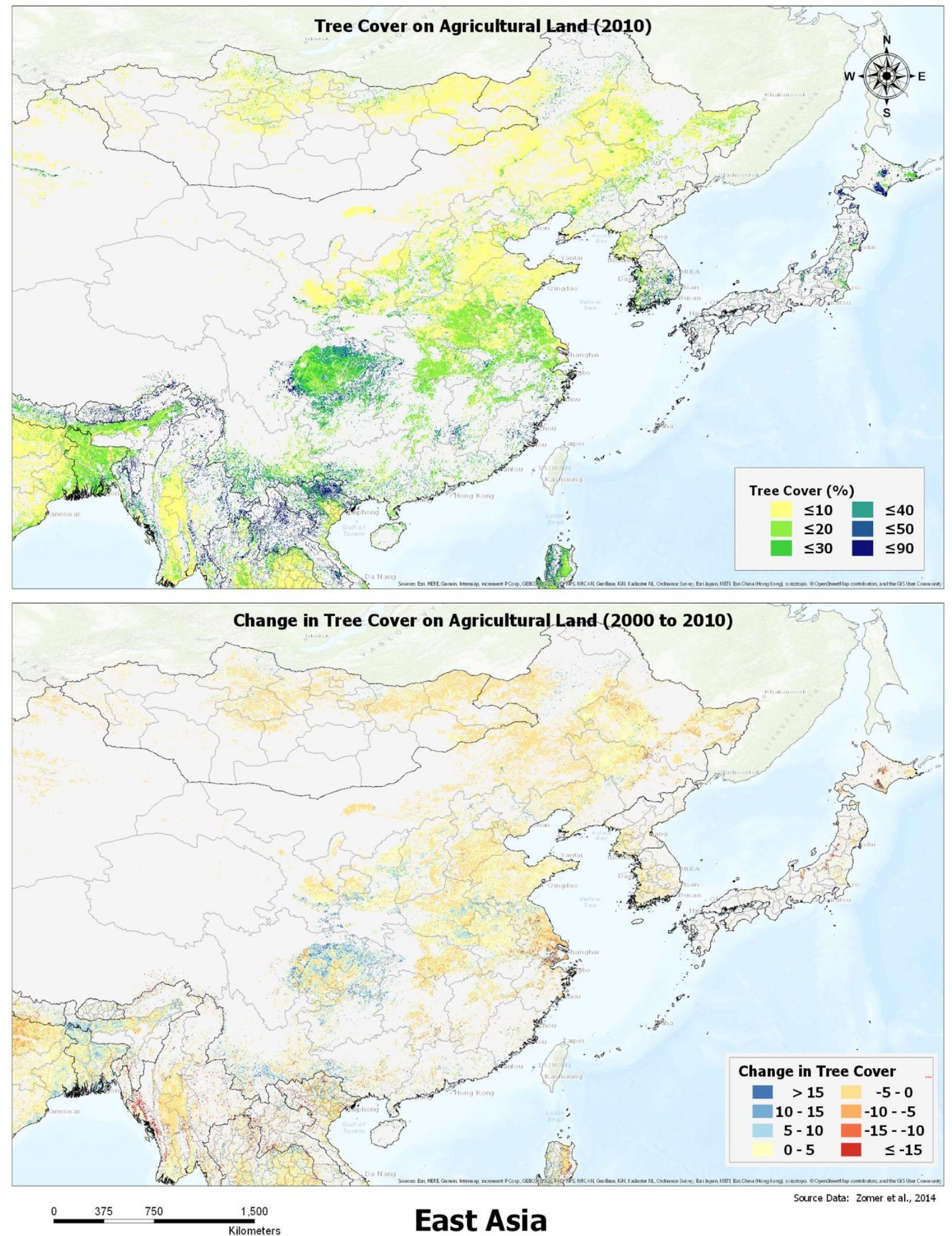

Figure 7. East Asia: Percent tree cover on agricultural land in the year 2010, and the change in percent tree cover on agricultural land from the year 2000 to 2010 

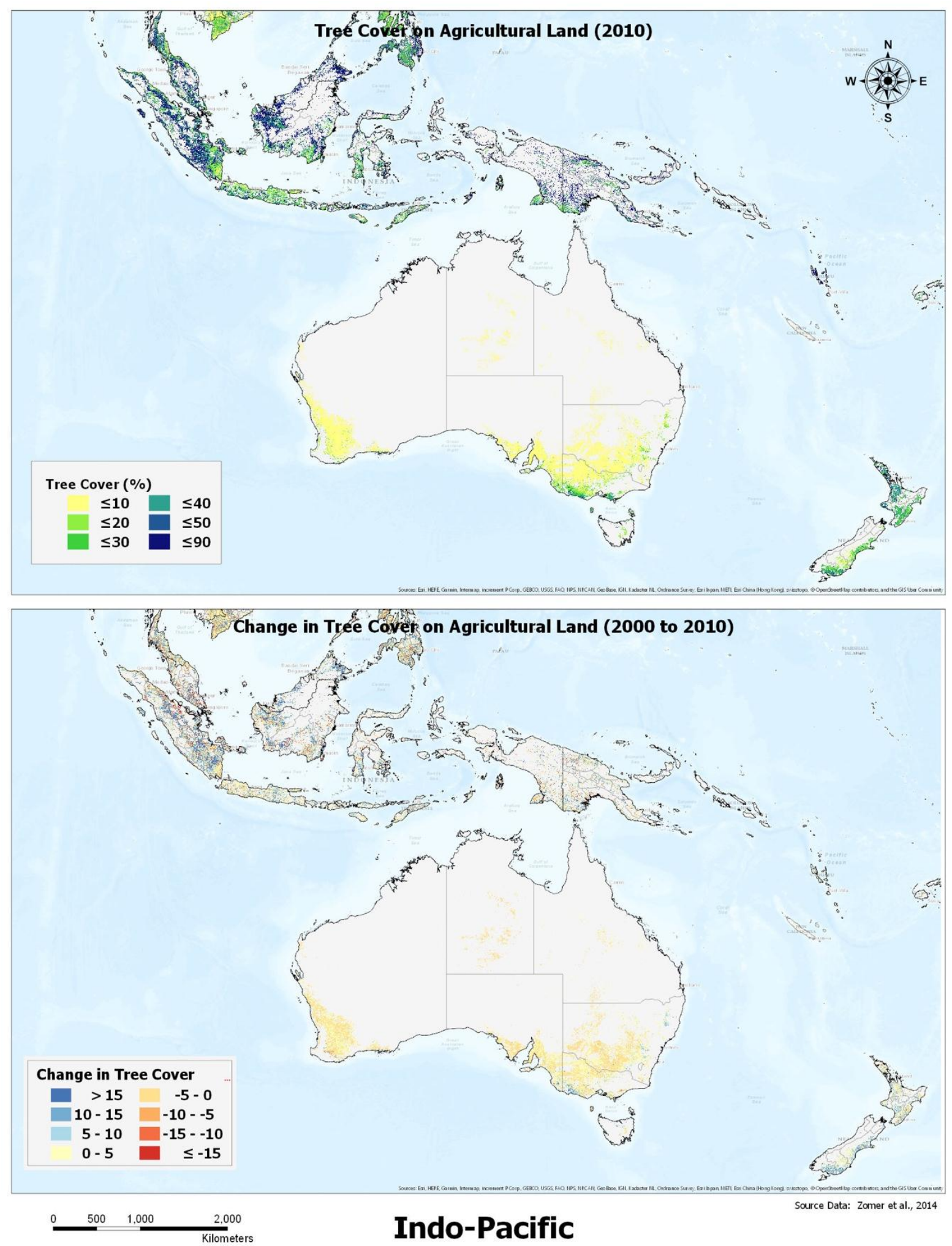

Figure 8. Indo-Pacific: Percent tree cover on agricultural land in the year 2010, and the change in percent tree cover on agricultural land from the year 2000 to 2010 

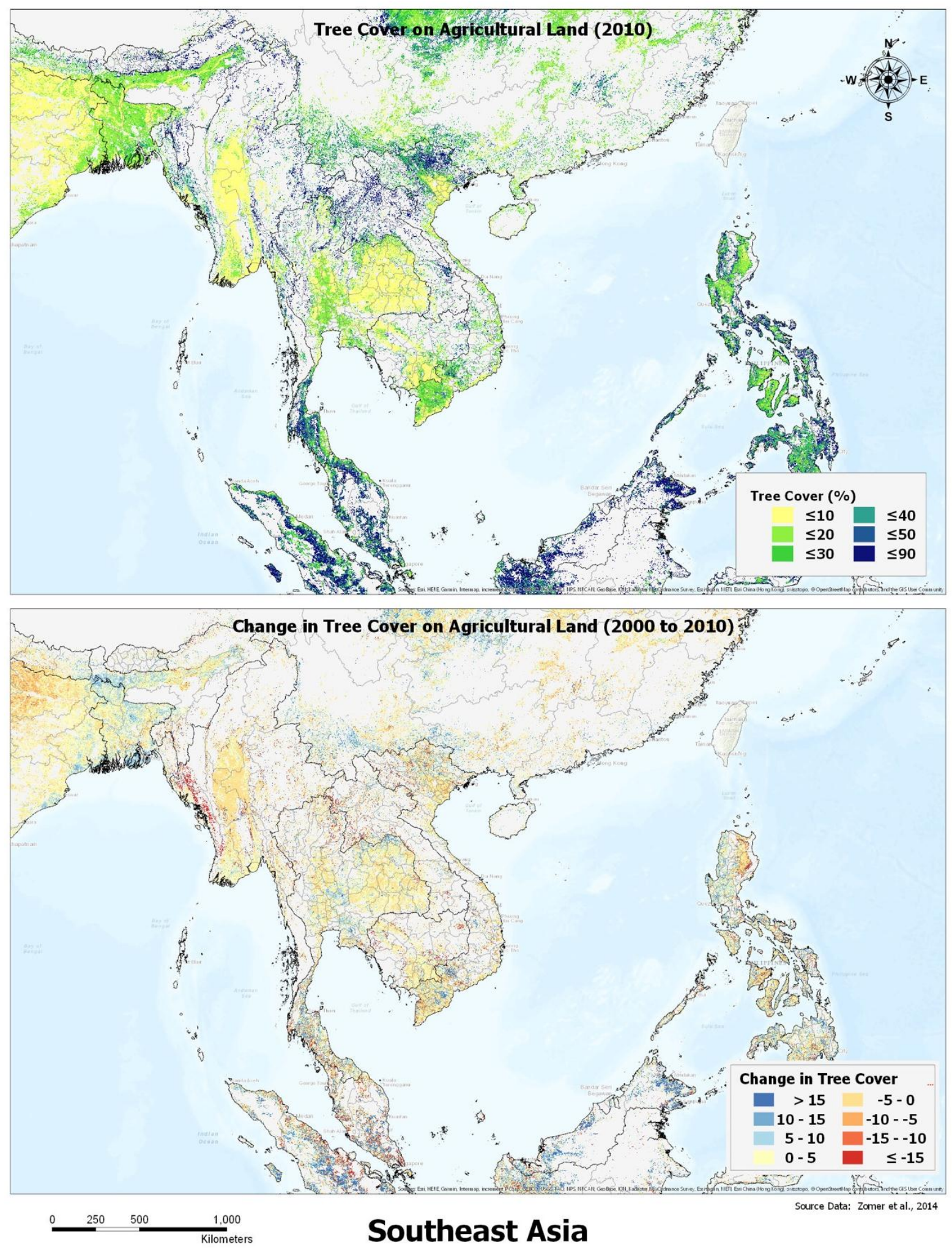

Figure 9. Southeast Asia: Percent tree cover on agricultural land in the year 2010, and the change in percent tree cover on agricultural land from the year 2000 to 2010 

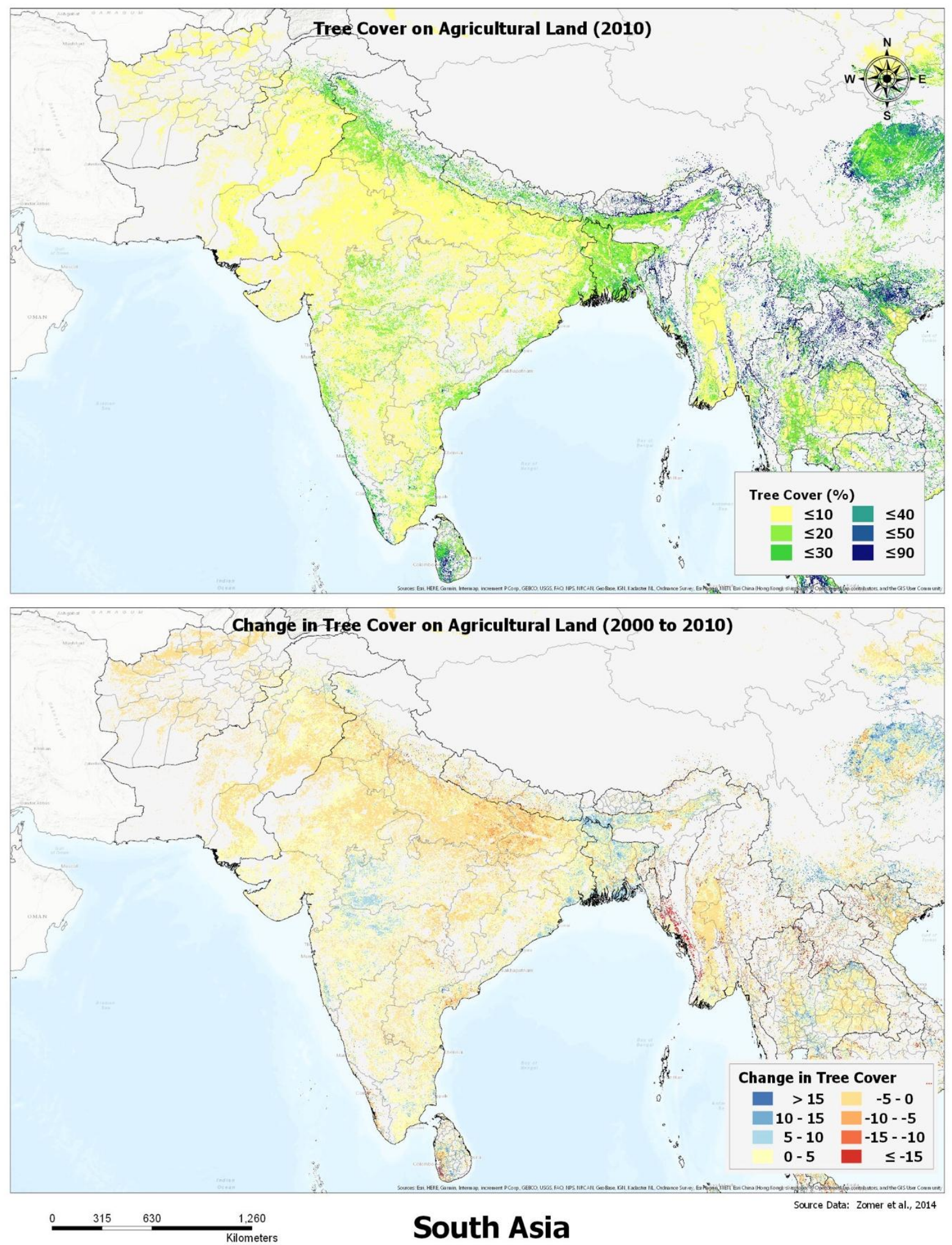

Figure 10. South Asia: Percent tree cover on agricultural land in the year 2010, and the change in percent tree cover on agricultural land from the year 2000 to 2010 


\section{Concluding remarks}

For the Asia-Pacific region as a whole, more than $44 \%$ of all agricultural land has at least $10 \%$ or more tree cover. The areal extent of agricultural land with greater than $10 \%$ tree cover increased over the decade from 2000 to 2010 by $4.3 \%$. Almost $18.4 \%$ of all agricultural land has at least $30 \%$ tree cover, representing 1.3 million $\mathrm{km}^{2}$ across the region.

Tree cover was found to be a significant component of agricultural land-use systems in most of the countries in the region, with the most arid, that is, Afghanistan, Pakistan, and Mongolia, having the least tree cover.

As shown, tree cover is a common feature on agricultural land throughout the Asia-Pacific region. It is therefore essential that this is recognized by all involved in agricultural production, planning and policy development.

Recognizing this existing important role of trees on farms and within agricultural landscapes, and by extension in agricultural systems, is ever more crucial as environmental and climatic change in the region is rapidly accelerating.

The role of trees for rural livelihoods, sustainability and resilience of agricultural systems, and for climate mitigation has brought agroforestry practices to the forefront of global sustainable development efforts.

The Asia Pacific region has a rich and diverse variety of historical and traditional agroforestry practices, as well as tree-planting programs that have been working with farmers and national agencies in some of the countries of the region for many decades.

The results of this report show that these efforts have had some success but that there is large potential for increasing tree cover within many of the countries of the region, which are below both regional and national averages.

Earlier research has shown that large-scale, tree-cover patterns cannot be fully explained by aridity, population density or bioclimatic region. This points towards the importance of other factors, such as tenure, markets, or other policies and institutional arrangements in affecting incentives for tree planting and management, as well as the historical trajectory that has led to the current pattern. Further investigations at finer national and sub-national scales are likely to prove even more illuminating in terms of understanding and evaluating the role and potential of trees in agricultural landscapes.

\section{Limitations}

This report is based upon remote-sensing data of tree cover and land cover, described as best available at the time. The characteristics, assumptions and limitations of the data layers and methods condition all the results. Zomer et al (2009) give an extensive overview of the limitations of both these data layers and our interpretation. 


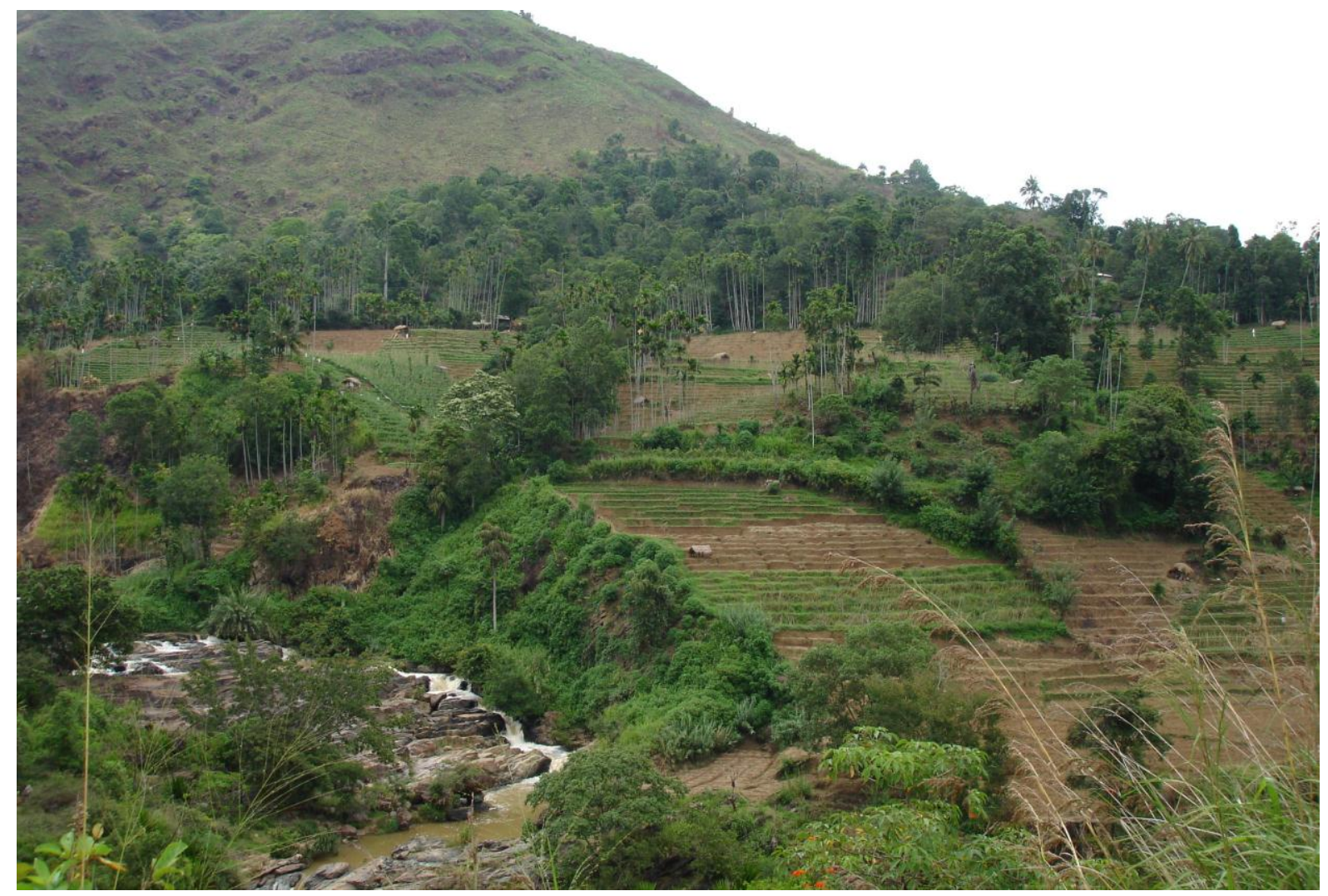

Photo 7. A paddy-based agroforestry system in Sri Lanka. Photo: World Agroforestry/DKNG Pushpakumara

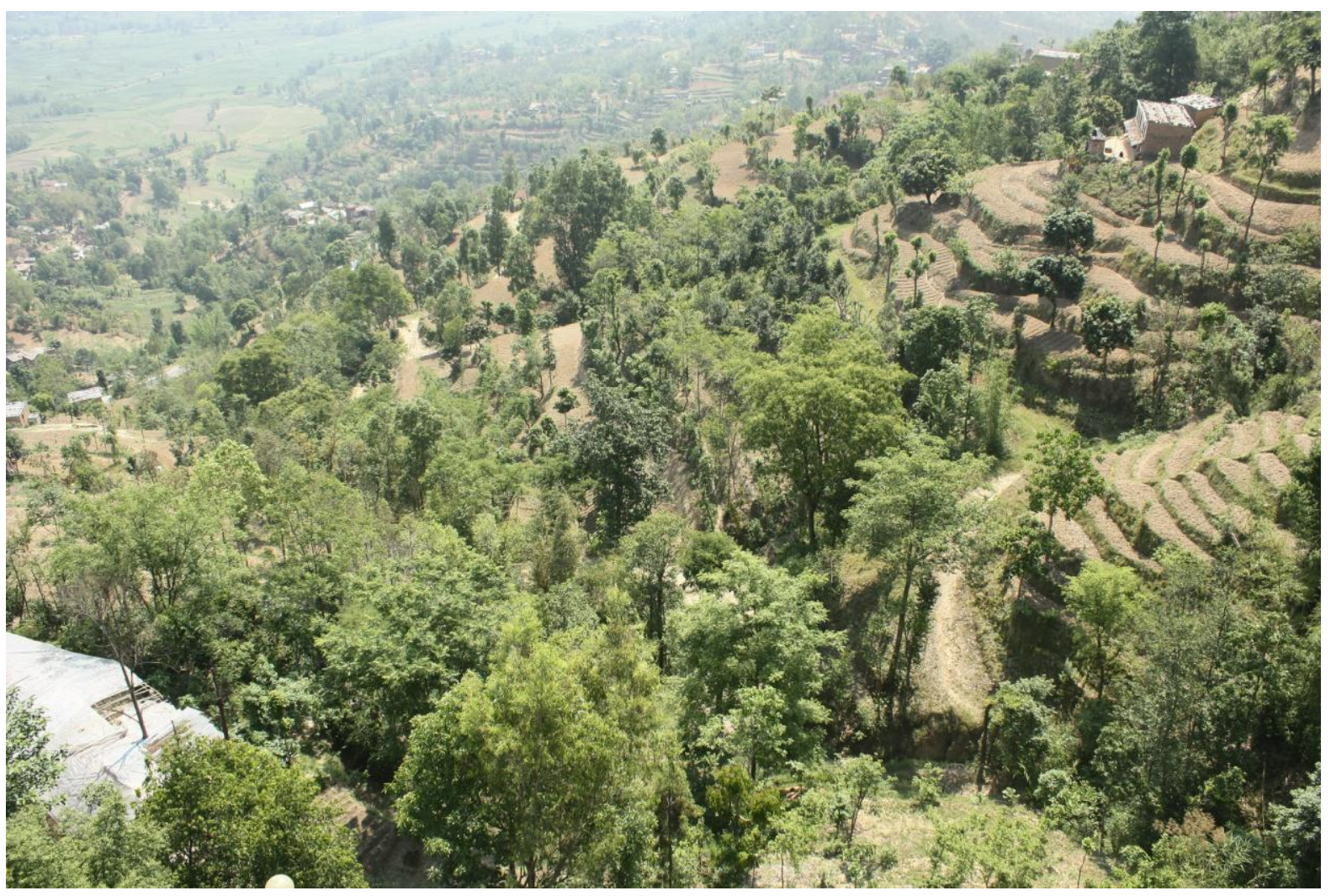

Photo 8. Tree in field integrated agroforestry system with animal husbandry, Nepal. Photo: World Agroforestry 


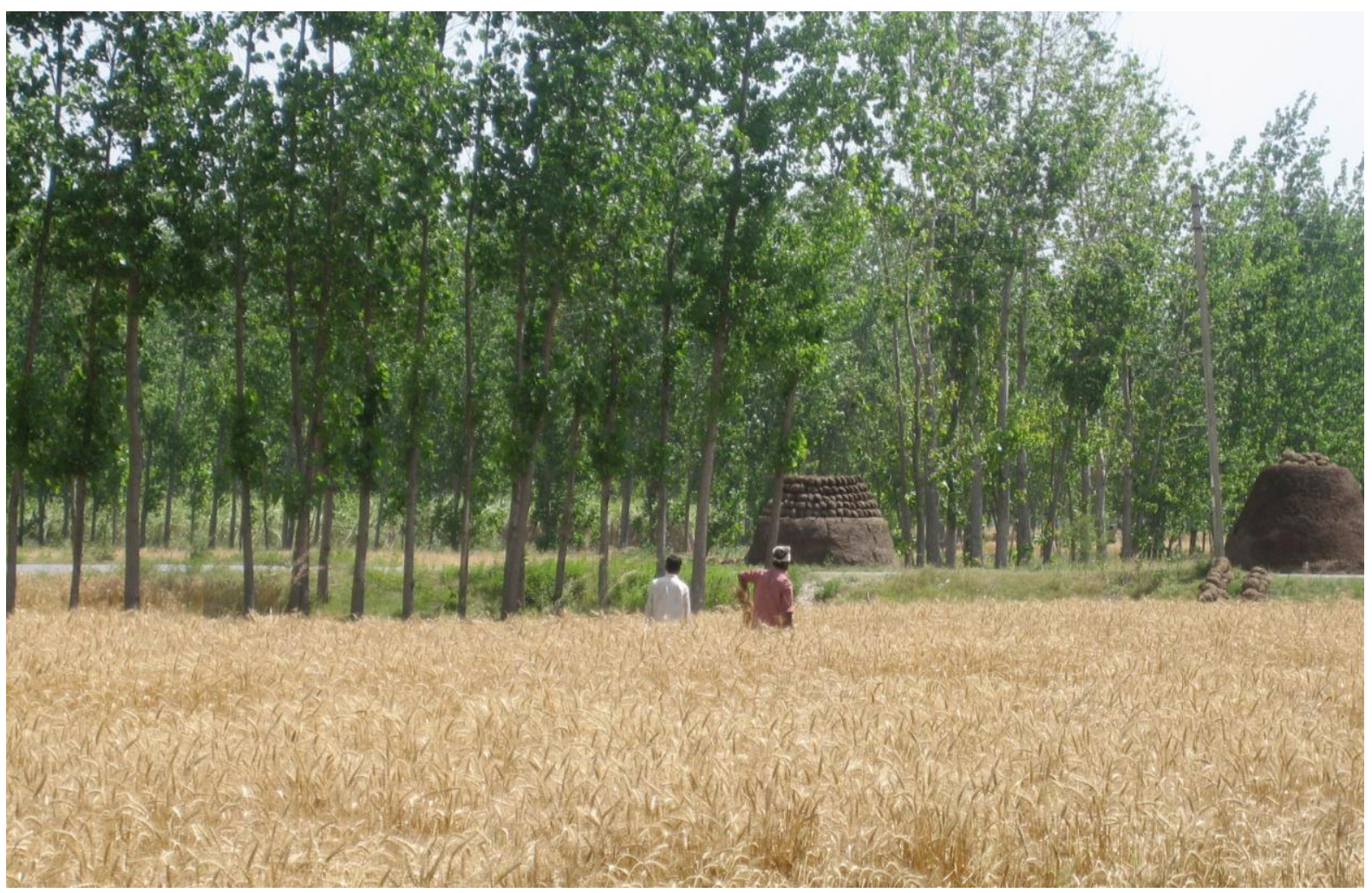

Photo 9. Poplar-wheat intercrop systems on irrigated farmland are found extensively across the northern parts of Haryana and Uttar Pradesh States, India. Photo: World Agroforestry/Robert Zomer

\section{References}

Bartholomé E. Belward AS. 2005. GLC2000: a new approach to global land cover mapping from Earth observation data. International Journal of Remote Sensing 26:1959-1977. DOI:10.1080/01431160412331291297.

De Foresta H, Somarriba E, Temu A, Boulanger D, Feuilly H, Gauthier M. 2013. Towards the assessment of trees outside forests. Resources Assessment Working Paper 183. Rome, Italy: Food and Agriculture Organization of the United Nations.

Di Miceli C, Sexton JO, Song XP, Feng M, Noojipady P, Anand A, Huang C, Kim DH, Collins KM, Channan S, Townshend JR. 2013. Global, 30-m resolution continuous fields of tree cover: Landsat-based rescaling of MODIS vegetation continuous fields with lidar-based estimates of error. International Journal of Digital Earth 6:427-448. DOI:10.1080/17538947.2013.786146.

Zomer RJ, Trabucco A, Coe R, Place F. 2009. Trees on farm: analysis of global extent and geographical patterns of agroforestry. ICRAF Working Paper 89. Nairobi, Kenya: World Agroforestry Centre. (ICRAF). http://www.worldagroforestry.org/downloads/Publications/PDFS/WP16263.pdf.

Zomer RJ, Trabucco A, Coe R, Place F, van Noordwijk M, Xu JC. 2014. Trees on farms: an update and reanalysis of agroforestry's global extent and socio-ecological characteristics. Working Paper 179. Bogor, Indonesia: World Agroforestry Centre (ICRAF) Southeast Asia Regional Program. DOI: 10.5716/ WP14064.

Zomer RJ, Neufeldt H, Xu J, Ahrends A, Bossio D, Trabucco A, van Noordwijk M, Wang M. 2016. Global tree cover and biomass carbon on agricultural land: the contribution of agroforestry to global and national carbon budgets. Scientific Reports 6:29987. DOI:10.1038/srep29987. 


\section{Annex}

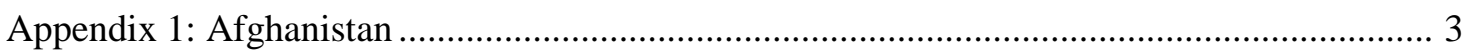

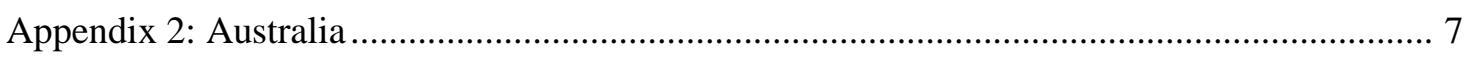

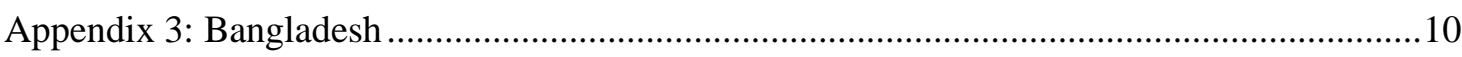

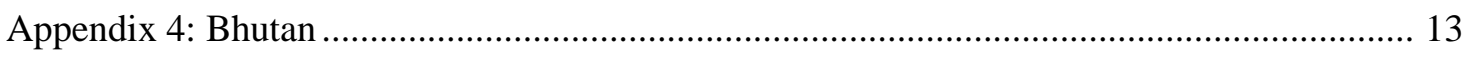

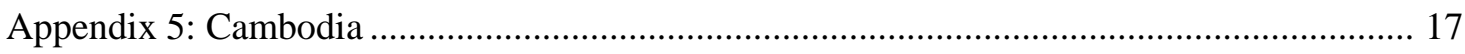

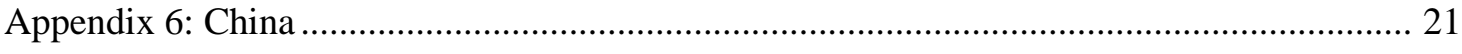

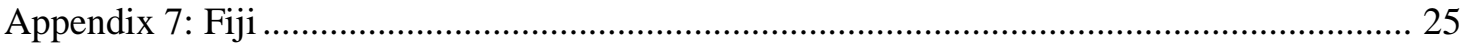

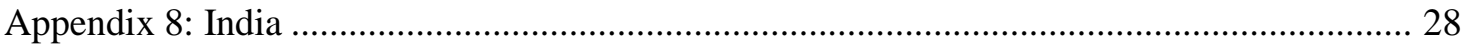

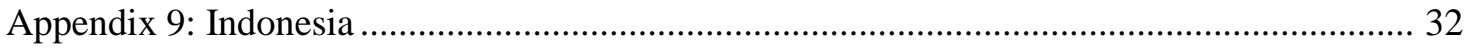

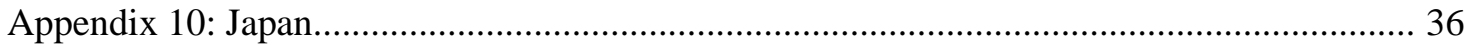

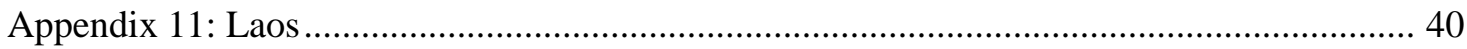

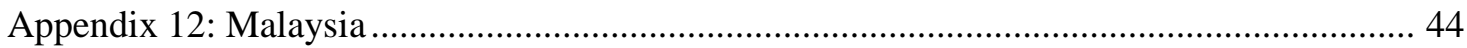

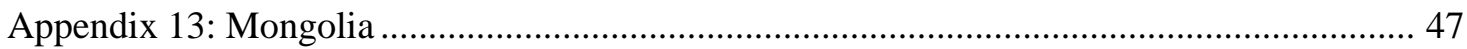

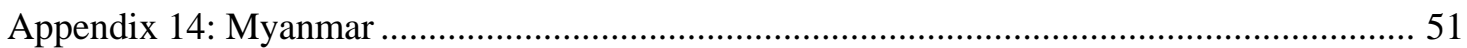

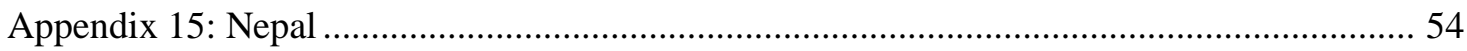

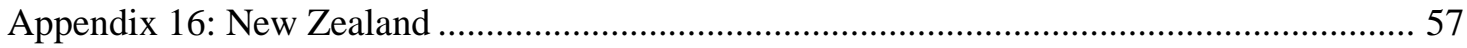

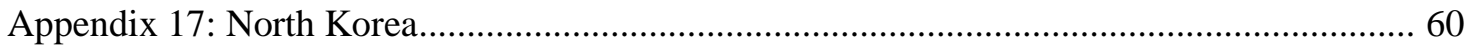

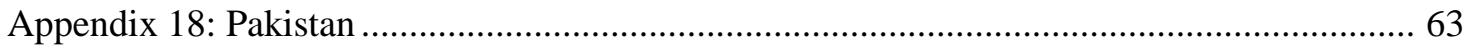

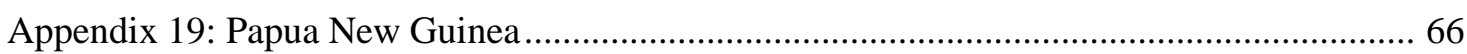

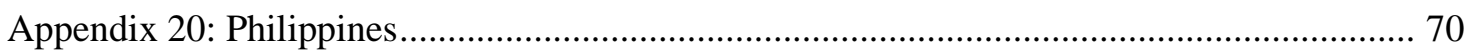

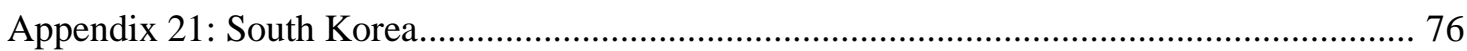

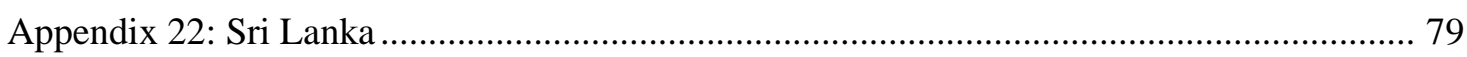

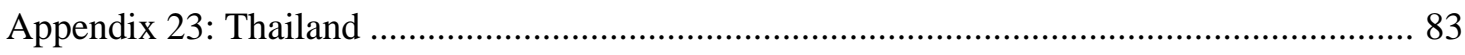

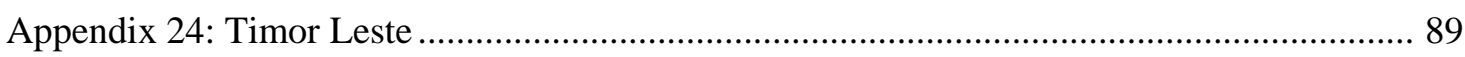

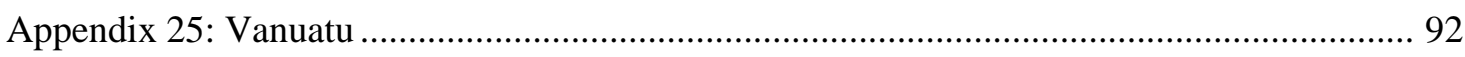

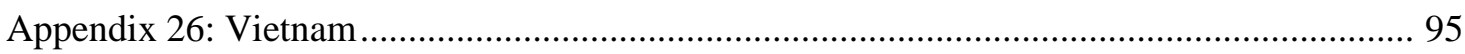


Appendix 1: Afghanistan

Average Percent Tree Cover by Province, and Areal Extent of Tree Cover Classes $\left(\mathrm{km}^{2}\right)$

\begin{tabular}{|c|c|c|c|c|c|c|c|c|c|c|c|c|c|c|c|c|}
\hline \multicolumn{2}{|c|}{ Afghanistan } & \multirow{2}{*}{\multicolumn{3}{|c|}{\begin{tabular}{|l} 
Average Tree Cover by Province \\
Mean Tree Cover Percent by Province
\end{tabular}}} & \multicolumn{12}{|c|}{ Areal Extent of Tree Cover Class $\left(\mathrm{km}^{2}\right)$} \\
\hline \multirow{3}{*}{ Province } & \multirow{3}{*}{ Area_km } & & & & \multirow{2}{*}{\multicolumn{4}{|c|}{$\begin{array}{c}\text { Year } 2000 \\
\text { Area by Tree Cover Class }\left(\mathrm{km}^{2}\right)\end{array}$}} & \multirow{2}{*}{\multicolumn{4}{|c|}{ Area by Tree Cover Class $\left(\mathrm{km}^{2}\right)$}} & \multirow{2}{*}{\multicolumn{4}{|c|}{$\begin{array}{c}\text { Change }(2000 \text { to } 2010) \\
\text { Area by Tree Cover Class }\left(\mathrm{km}^{2}\right)\end{array}$}} \\
\hline & & & (\%) & & & & & & & & & & & & & \\
\hline & & 2000 & 2010 & Change & $<10 \%$ & $10-20 \%$ & $20-30 \%$ & $>30 \%$ & $<10 \%$ & $10-20 \%$ & $20-30 \%$ & $>30 \%$ & $<10 \%$ & $10-20 \%$ & $20-30 \%$ & $>30 \%$ \\
\hline Badakhshan & 1,913 & 1.03 & 1.29 & 0.26 & 1,913 & 0 & 0 & 0 & 1,913 & 0 & $\overline{0}$ & $\overline{0}$ & 0 & 0 & 0 & $\overline{0}$ \\
\hline Badghis & 9,209 & 0.04 & 0.05 & 0.01 & 9,209 & 0 & 0 & 0 & 9,209 & 0 & 0 & 0 & 0 & 0 & 0 & 0 \\
\hline Baghlan & 5,289 & 0.38 & 0.53 & 0.15 & 5,289 & 0 & 0 & 0 & 5,289 & 0 & 0 & 0 & 0 & 0 & 0 & 0 \\
\hline Balkh & 7,145 & 0.19 & 0.26 & 0.07 & 7,145 & 0 & 0 & 0 & 7,143 & 2 & 0 & 0 & -2 & 2 & 0 & 0 \\
\hline Bamyan & 227 & 0.19 & 0.29 & 0.10 & 227 & 0 & 0 & 0 & 227 & 0 & 0 & 0 & 0 & 0 & 0 & 0 \\
\hline Daykundi & 2,646 & 0.11 & 0.17 & 0.06 & 2,646 & 0 & 0 & 0 & 2,646 & 0 & 0 & 0 & 0 & 0 & 0 & 0 \\
\hline Farah & 7,810 & 0.02 & 0.04 & 0.01 & 7,810 & 0 & 0 & 0 & 7,810 & 0 & 0 & 0 & 0 & 0 & 0 & 0 \\
\hline Faryab & 8,032 & 0.04 & 0.05 & 0.00 & 8,032 & 0 & 0 & 0 & 8,032 & 0 & 0 & 0 & 0 & 0 & 0 & 0 \\
\hline Ghazni & 2,465 & 0.05 & 0.14 & 0.09 & 2,465 & 0 & 0 & 0 & 2,463 & 2 & 0 & 0 & -2 & 2 & 0 & 0 \\
\hline Ghor & 3,965 & 0.02 & 0.05 & 0.04 & 3,965 & 0 & 0 & 0 & 3,965 & 0 & 0 & 0 & 0 & 0 & 0 & 0 \\
\hline Hilmand & 6,471 & 0.24 & 0.31 & 0.07 & 6,471 & 0 & 0 & 0 & 6,471 & 0 & 0 & 0 & 0 & 0 & 0 & 0 \\
\hline Hirat & 13,929 & 0.07 & 0.14 & 0.07 & 13,929 & 0 & 0 & 0 & 13,929 & 0 & 0 & 0 & 0 & 0 & 0 & 0 \\
\hline Jawzjan & 3,342 & 0.11 & 0.18 & 0.07 & 3,342 & 0 & 0 & 0 & 3,337 & 5 & 0 & 0 & -5 & 5 & 0 & 0 \\
\hline Kabul & 1,239 & 0.38 & 0.67 & 0.29 & 1,236 & 1 & 2 & 0 & 1,236 & 3 & 0 & 0 & 0 & 2 & -2 & 0 \\
\hline Kandahar & 5,168 & 0.05 & 0.14 & 0.08 & 5,168 & 0 & 0 & 0 & 5,168 & 0 & 0 & 0 & 0 & 0 & 0 & 0 \\
\hline Kapisa & 805 & 1.16 & 1.64 & 0.48 & 802 & 2 & 1 & 0 & 802 & 3 & 0 & 0 & 0 & 1 & -1 & 0 \\
\hline Khost & 1,524 & 2.03 & 2.81 & 0.78 & 1,476 & 42 & 5 & 1 & 1,487 & 34 & 3 & 0 & 11 & -8 & -2 & -1 \\
\hline Kunar & 1,430 & 6.78 & 8.46 & 1.67 & 1,115 & 216 & 85 & 14 & 1,052 & 257 & 90 & 31 & -63 & 41 & 5 & 17 \\
\hline Kunduz & 3,321 & 1.44 & 1.80 & 0.36 & 3,302 & 19 & 0 & 0 & 3,310 & 11 & 0 & 0 & 8 & -8 & 0 & 0 \\
\hline Laghman & 1,506 & 2.72 & 3.15 & 0.42 & 1,394 & 80 & 24 & 8 & 1,391 & 79 & 25 & 11 & -3 & -1 & 1 & 3 \\
\hline Logar & 347 & 0.44 & 0.65 & 0.21 & 347 & 0 & 0 & 0 & 347 & 0 & 0 & 0 & 0 & 0 & 0 & 0 \\
\hline Nangarhar & 3,271 & 1.61 & 2.34 & 0.73 & 3,177 & 59 & 31 & 4 & 3,152 & 84 & 28 & 7 & -25 & 25 & -3 & 3 \\
\hline Nimroz & 1,017 & 0.03 & 0.43 & 0.41 & 1,017 & 0 & 0 & 0 & 1,017 & 0 & 0 & 0 & 0 & 0 & 0 & 0 \\
\hline Nuristan & 496 & 11.22 & 11.74 & 0.52 & 285 & 115 & 69 & 27 & 282 & 111 & 71 & 32 & -3 & -4 & 2 & 5 \\
\hline Paktika & 1,656 & 0.25 & 0.33 & 0.08 & 1,645 & 11 & 0 & 0 & 1,648 & 8 & 0 & 0 & 3 & -3 & 0 & 0 \\
\hline Paktya & 1,093 & 2.19 & 2.59 & 0.40 & 1,054 & 35 & 4 & 0 & 1,056 & 35 & 2 & 0 & 2 & 0 & -2 & 0 \\
\hline Panjshir & 308 & 1.00 & 1.28 & 0.28 & 308 & 0 & 0 & 0 & 308 & 0 & 0 & 0 & 0 & 0 & 0 & 0 \\
\hline Parwan & 910 & 1.35 & 1.89 & 0.54 & 910 & 0 & 0 & 0 & 908 & 2 & 0 & 0 & -2 & 2 & 0 & 0 \\
\hline Samangan & 3,426 & 0.04 & 0.04 & 0.00 & 3,426 & 0 & 0 & 0 & 3,426 & 0 & 0 & 0 & 0 & 0 & 0 & 0 \\
\hline Sari Pul & 4,604 & 0.11 & 0.09 & -0.02 & 4,604 & 0 & 0 & 0 & 4,604 & 0 & 0 & 0 & 0 & 0 & 0 & 0 \\
\hline Takhar & 4,901 & 0.71 & 0.82 & 0.11 & 4,891 & 10 & 0 & 0 & 4,889 & 12 & 0 & 0 & -2 & 2 & 0 & 0 \\
\hline Uruzgan & 5,479 & 0.10 & 0.15 & 0.05 & 5,479 & 0 & 0 & 0 & 5,479 & 0 & 0 & 0 & 0 & 0 & 0 & 0 \\
\hline Wardak & 537 & 0.21 & 0.39 & 0.18 & 537 & 0 & 0 & 0 & 537 & 0 & 0 & 0 & 0 & 0 & 0 & 0 \\
\hline Zabul & 3,483 & 0.02 & 0.05 & 0.03 & 3,483 & 0 & 0 & 0 & 3,483 & 0 & 0 & 0 & 0 & 0 & 0 & 0 \\
\hline Afghanistan - All & 118,964 & 0.45 & 0.58 & 0.13 & 118,100 & 590 & 221 & 54 & 118,017 & 648 & 219 & 81 & -83 & 58 & -2 & 27 \\
\hline
\end{tabular}




\begin{tabular}{|c|c|c|c|c|c|c|c|c|c|c|c|c|c|c|c|c|}
\hline \multicolumn{2}{|c|}{ Afghanistan } & \multirow{3}{*}{\multicolumn{3}{|c|}{$\begin{array}{c}\text { Average Tree Cover by Province } \\
\text { Percent Tree Cover } \\
(\%)\end{array}$}} & \multicolumn{12}{|c|}{ Areal Extent of Tree Cover Class as a Percent of Total Agricultural Area (\%) } \\
\hline \multirow{2}{*}{ Province } & \multirow{2}{*}{ Area_km } & & & & \multirow{2}{*}{\multicolumn{4}{|c|}{$\begin{array}{c}\text { Year } 2000 \\
\text { Percent of Total Agrciultural Area (\%) }\end{array}$}} & \multirow{2}{*}{\multicolumn{4}{|c|}{$\begin{array}{c}\text { Year } 2010 \\
\text { Percent of Total Agrciultural Area (\%) }\end{array}$}} & & Change (2 & 0 to 2010$)$ & \\
\hline & & & & & Per & tof lotal A & & & & & & & \multicolumn{4}{|c|}{ Percent of Total Agrciultural Area (\%) } \\
\hline Badakhshan & 1.913 & 103 & & 0.26 & 10000 & 0.000 & $20-30 \%$ & $>30 \%$ & $<10 \%$ & $10-20 \%$ & $20-30 \%$ & $>30 \%$ & $<10 \%$ & $10-20 \%$ & $20-30 \%$ & $>30 \%$ \\
\hline Badghis & 9,209 & 0.04 & 0.05 & 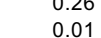 & 100.00 & 0.00 & 0.00 & 0.00 & 100.00 & 0.00 & 0.00 & 0.00 & 0.00 & 0.00 & 0.00 & 0.00 \\
\hline Baghlan & 5,289 & 0.38 & 0.53 & 0.15 & 100.00 & 0.00 & 0.00 & 0.00 & 100.00 & 0.00 & 0.00 & 0.00 & 0.00 & 0.00 & 0.00 & 0.00 \\
\hline Balkh & 7,145 & 0.19 & 0.26 & 0.07 & 100.00 & 0.00 & 0.00 & 0.00 & 99.97 & 0.03 & 0.00 & 0.00 & -0.03 & 0.03 & 0.00 & 0.00 \\
\hline Bamyan & 227 & 0.19 & 0.29 & 0.10 & 100.00 & 0.00 & 0.00 & 0.00 & 100.00 & 0.00 & 0.00 & 0.00 & 0.00 & 0.00 & 0.00 & 0.00 \\
\hline Daykundi & 2,646 & 0.11 & 0.17 & 0.06 & 100.00 & 0.00 & 0.00 & 0.00 & 100.00 & 0.00 & 0.00 & 0.00 & 0.00 & 0.00 & 0.00 & 0.00 \\
\hline Farah & 7,810 & 0.02 & 0.04 & 0.01 & 100.00 & 0.00 & 0.00 & 0.00 & 100.00 & 0.00 & 0.00 & 0.00 & 0.00 & 0.00 & 0.00 & 0.00 \\
\hline Faryab & 8,032 & 0.04 & 0.05 & 0.00 & 100.00 & 0.00 & 0.00 & 0.00 & 100.00 & 0.00 & 0.00 & 0.00 & 0.00 & 0.00 & 0.00 & 0.00 \\
\hline Ghazni & 2,465 & 0.05 & 0.14 & 0.09 & 100.00 & 0.00 & 0.00 & 0.00 & 99.92 & 0.08 & 0.00 & 0.00 & -0.08 & 0.08 & 0.00 & 0.00 \\
\hline Ghor & 3,965 & 0.02 & 0.05 & 0.04 & 100.00 & 0.00 & 0.00 & 0.00 & 100.00 & 0.00 & 0.00 & 0.00 & 0.00 & 0.00 & 0.00 & 0.00 \\
\hline Hilmand & 6,471 & 0.24 & 0.31 & 0.07 & 100.00 & 0.00 & 0.00 & 0.00 & 100.00 & 0.00 & 0.00 & 0.00 & 0.00 & 0.00 & 0.00 & 0.00 \\
\hline Hirat & 13,929 & 0.07 & 0.14 & 0.07 & 100.00 & 0.00 & 0.00 & 0.00 & 100.00 & 0.00 & 0.00 & 0.00 & 0.00 & 0.00 & 0.00 & 0.00 \\
\hline Jawzjan & 3,342 & 0.11 & 0.18 & 0.07 & 100.00 & 0.00 & 0.00 & 0.00 & 99.85 & 0.15 & 0.00 & 0.00 & -0.15 & 0.15 & 0.00 & 0.00 \\
\hline Kabul & 1,239 & 0.38 & 0.67 & 0.29 & 99.76 & 0.08 & 0.16 & 0.00 & 99.76 & 0.24 & 0.00 & 0.00 & 0.00 & 0.16 & -0.16 & 0.00 \\
\hline Kandahar & 5,168 & 0.05 & 0.14 & 0.08 & 100.00 & 0.00 & 0.00 & 0.00 & 100.00 & 0.00 & 0.00 & 0.00 & 0.00 & 0.00 & 0.00 & 0.00 \\
\hline Kapisa & 805 & 1.16 & 1.64 & 0.48 & 99.63 & 0.25 & 0.12 & 0.00 & 99.63 & 0.37 & 0.00 & 0.00 & 0.00 & 0.12 & -0.12 & 0.00 \\
\hline Khost & 1,524 & 2.03 & 2.81 & 0.78 & 96.85 & 2.76 & 0.33 & 0.07 & 97.57 & 2.23 & 0.20 & 0.00 & 0.72 & -0.52 & -0.13 & -0.07 \\
\hline Kunar & 1,430 & 6.78 & 8.46 & 1.67 & 77.97 & 15.11 & 5.94 & 0.98 & 73.57 & 17.97 & 6.29 & 2.17 & -4.41 & 2.87 & 0.35 & 1.19 \\
\hline Kunduz & 3,321 & 1.44 & 1.80 & 0.36 & 99.43 & 0.57 & 0.00 & 0.00 & 99.67 & 0.33 & 0.00 & 0.00 & 0.24 & -0.24 & 0.00 & 0.00 \\
\hline Laghman & 1,506 & 2.72 & 3.15 & 0.42 & 92.56 & 5.31 & 1.59 & 0.53 & 92.36 & 5.25 & 1.66 & 0.73 & -0.20 & -0.07 & 0.07 & 0.20 \\
\hline Logar & 347 & 0.44 & 0.65 & 0.21 & 100.00 & 0.00 & 0.00 & 0.00 & 100.00 & 0.00 & 0.00 & 0.00 & 0.00 & 0.00 & 0.00 & 0.00 \\
\hline Nangarhar & 3,271 & 1.61 & 2.34 & 0.73 & 97.13 & 1.80 & 0.95 & 0.12 & 96.36 & 2.57 & 0.86 & 0.21 & -0.76 & 0.76 & -0.09 & 0.09 \\
\hline Nimroz & 1,017 & 0.03 & 0.43 & 0.41 & 100.00 & 0.00 & 0.00 & 0.00 & 100.00 & 0.00 & 0.00 & 0.00 & 0.00 & 0.00 & 0.00 & 0.00 \\
\hline Nuristan & 496 & 11.22 & 11.74 & 0.52 & 57.46 & 23.19 & 13.91 & 5.44 & 56.86 & 22.38 & 14.31 & 6.45 & -0.60 & -0.81 & 0.40 & 1.01 \\
\hline Paktika & 1,656 & 0.25 & 0.33 & 0.08 & 99.34 & 0.66 & 0.00 & 0.00 & 99.52 & 0.48 & 0.00 & 0.00 & 0.18 & -0.18 & 0.00 & 0.00 \\
\hline Paktya & 1,093 & 2.19 & 2.59 & 0.40 & 96.43 & 3.20 & 0.37 & 0.00 & 96.62 & 3.20 & 0.18 & 0.00 & 0.18 & 0.00 & -0.18 & 0.00 \\
\hline Panjshir & 308 & 1.00 & 1.28 & 0.28 & 100.00 & 0.00 & 0.00 & 0.00 & 100.00 & 0.00 & 0.00 & 0.00 & 0.00 & 0.00 & 0.00 & 0.00 \\
\hline Parwan & 910 & 1.35 & 1.89 & 0.54 & 100.00 & 0.00 & 0.00 & 0.00 & 99.78 & 0.22 & 0.00 & 0.00 & -0.22 & 0.22 & 0.00 & 0.00 \\
\hline Samangan & 3,426 & 0.04 & 0.04 & 0.00 & 100.00 & 0.00 & 0.00 & 0.00 & 100.00 & 0.00 & 0.00 & 0.00 & 0.00 & 0.00 & 0.00 & 0.00 \\
\hline Sari Pul & 4,604 & 0.11 & 0.09 & -0.02 & 100.00 & 0.00 & 0.00 & 0.00 & 100.00 & 0.00 & 0.00 & 0.00 & 0.00 & 0.00 & 0.00 & 0.00 \\
\hline Takhar & 4,901 & 0.71 & 0.82 & 0.11 & 99.80 & 0.20 & 0.00 & 0.00 & 99.76 & 0.24 & 0.00 & 0.00 & -0.04 & 0.04 & 0.00 & 0.00 \\
\hline Uruzgan & 5,479 & 0.10 & 0.15 & 0.05 & 100.00 & 0.00 & 0.00 & 0.00 & 100.00 & 0.00 & 0.00 & 0.00 & 0.00 & 0.00 & 0.00 & 0.00 \\
\hline Wardak & 537 & 0.21 & 0.39 & 0.18 & 100.00 & 0.00 & 0.00 & 0.00 & 100.00 & 0.00 & 0.00 & 0.00 & 0.00 & 0.00 & 0.00 & 0.00 \\
\hline Zabul & 3,483 & 0.02 & 0.05 & 0.03 & 100.00 & 0.00 & 0.00 & 0.00 & 100.00 & 0.00 & 0.00 & 0.00 & 0.00 & 0.00 & 0.00 & 0.00 \\
\hline Afghanistan - All & 118,964 & 0.45 & 0.58 & 0.13 & 99.27 & 0.50 & 0.19 & 0.05 & 99.20 & 0.54 & 0.18 & 0.07 & -0.07 & 0.05 & 0.00 & 0.02 \\
\hline
\end{tabular}


Asia Pacific Region : Tree Cover on Agricultural Land (2010)

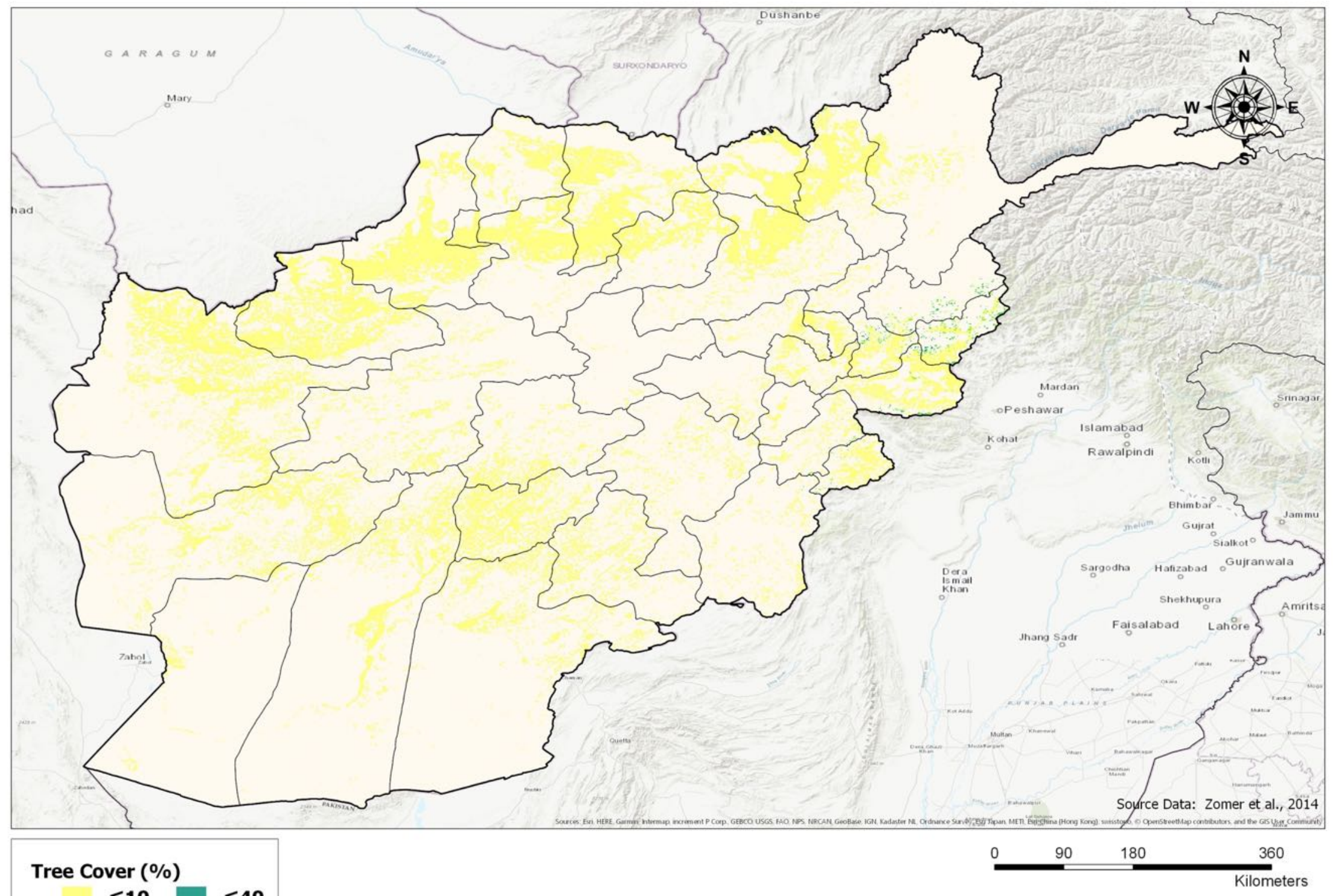
$\leq \mathbf{1 0}$

Afghanistan 
Asia Pacific Region : Change in Tree Cover on Agricultural Land (2000 - 2010)

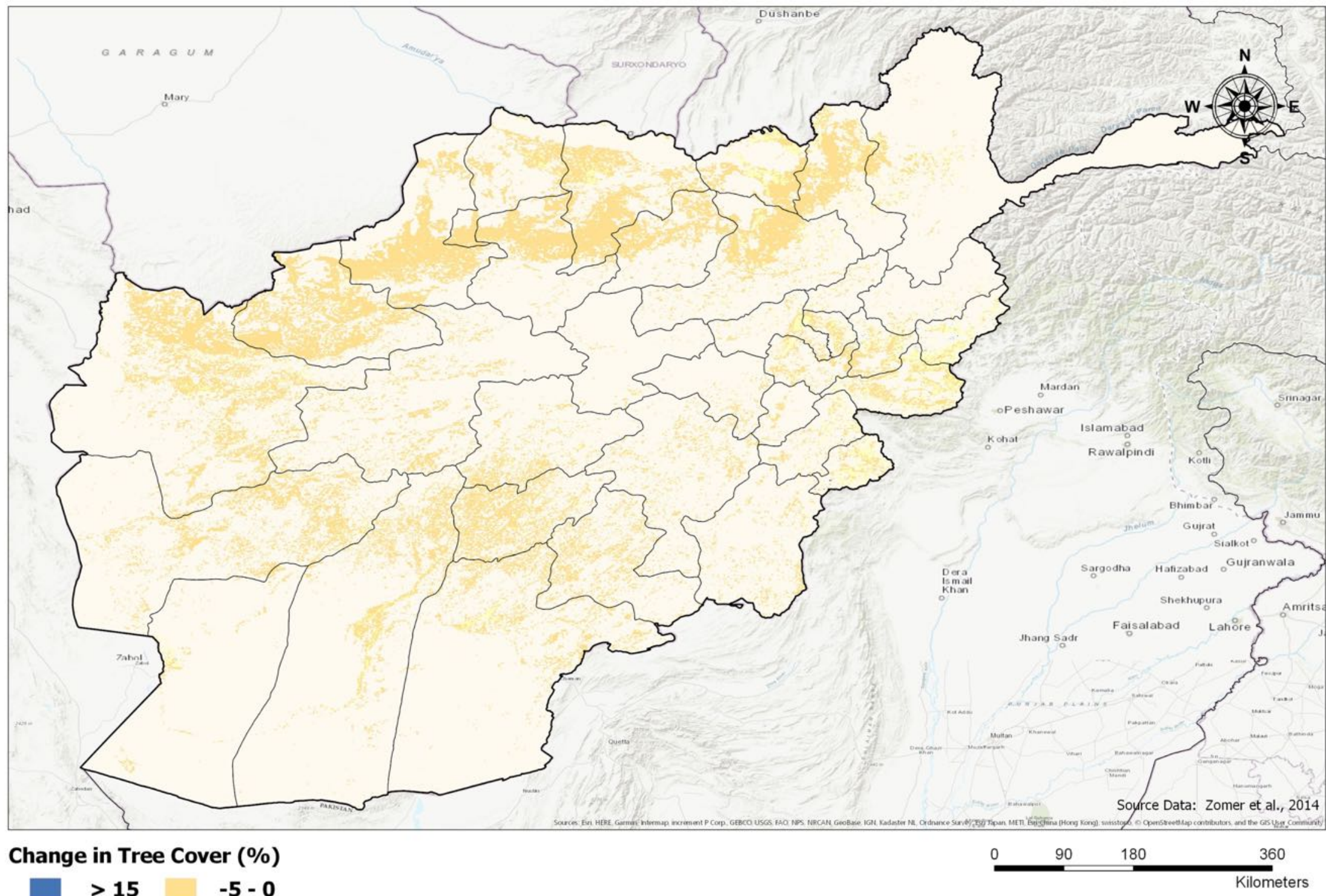

\begin{tabular}{|r|r|}
$>15$ & $-5-0$ \\
$10-15$ & $-10--5$ \\
$5-10$ & $-15--10$ \\
$0-5$ & $\leq-15$
\end{tabular}

Afghanistan 


\section{Appendix 2: Australia}

Average Percent Tree Cover by Province, and Areal Extent of Tree Cover Classes $\left(\mathrm{km}^{2}\right)$

\begin{tabular}{|c|c|c|c|c|c|c|c|c|c|c|c|c|c|c|c|c|}
\hline \multicolumn{2}{|l|}{ Australia } & \multicolumn{3}{|c|}{ Average Tree Cover by Province } & \multicolumn{12}{|c|}{ Areal Extent of Tree Cover Class $\left(\mathrm{km}^{2}\right)$} \\
\hline \multirow{3}{*}{ State } & \multirow{3}{*}{ Area_km } & \multirow{2}{*}{\multicolumn{3}{|c|}{$\begin{array}{c}\text { Mean Tree Cover Percent by Province } \\
(\%)\end{array}$}} & \multirow{2}{*}{\multicolumn{4}{|c|}{$\begin{array}{c}\text { Year } 2000 \\
\text { Area by Tree Cover Class }\left(\mathrm{km}^{2}\right)\end{array}$}} & \multirow{2}{*}{\multicolumn{4}{|c|}{$\begin{array}{c}\text { Year } 2010 \\
\text { Area by Tree Cover Class }\left(\mathrm{km}^{2}\right)\end{array}$}} & \multirow{2}{*}{\multicolumn{4}{|c|}{$\begin{array}{c}\text { Change }(2000 \text { to } 2010) \\
\text { Area by Tree Cover Class }\left(\mathrm{km}^{2}\right)\end{array}$}} \\
\hline & & & & & & & & & & & & & & & & \\
\hline & & 2000 & 2010 & Change & $<10 \%$ & $10-20 \%$ & $20-30 \%$ & $>30 \%$ & $<10 \%$ & $10-20 \%$ & $20-30 \%$ & $>30 \%$ & $<10 \%$ & $10-20 \%$ & $20-30 \%$ & $>30 \%$ \\
\hline Australian Capital Territory & 61 & 5.80 & 7.13 & 1.33 & 56 & 4 & 1 & 0 & 56 & 4 & 1 & 0 & $\overline{0}$ & 0 & 0 & 0 \\
\hline New South Wales & 185,128 & 3.79 & 4.55 & 0.77 & 178,554 & 5,568 & 723 & 284 & 169,044 & 14,135 & 1,427 & 523 & $-9,510$ & 8,567 & 704 & 239 \\
\hline Northern Territory & 29,548 & 3.55 & 1.70 & -1.85 & 29,506 & 41 & 1 & 0 & 29,548 & 0 & 0 & 0 & 42 & -41 & -1 & 0 \\
\hline Queensland & 22,223 & 2.51 & 3.25 & 0.74 & 22,009 & 105 & 41 & 68 & 22,025 & 93 & 45 & 60 & 16 & -12 & 4 & -8 \\
\hline South Australia & 99,836 & 4.49 & 5.18 & 9 & 90,618 & 7,559 & 1,201 & 459 & 88,090 & 8,524 & 2,213 & 1,010 & $-2,528$ & 965 & 1,012 & 551 \\
\hline Tasmania & 4,742 & 18.42 & 21.69 & 3.28 & 1,169 & 2,174 & 636 & 763 & 619 & 2,031 & 996 & 1,096 & -550 & -143 & 360 & 333 \\
\hline Victoria & 108,917 & 8.32 & 9.92 & 1.60 & 78,966 & 19,464 & 6,634 & 3,854 & 73,608 & 20,554 & 7,891 & 6,865 & $-5,358$ & 1,090 & 1,257 & 3,011 \\
\hline Western Australia & 147,418 & 3.05 & 3.37 & 0.31 & 139,409 & 6,428 & 1,291 & 291 & 139,122 & 5,937 & 1,668 & 692 & -287 & -491 & 377 & 401 \\
\hline Australia - All & 597,873 & 4.61 & 5.29 & 0.68 & 540,287 & 41,343 & 10,528 & 5,719 & 522,112 & 51,278 & 14,241 & 10,246 & $-18,175$ & 9,935 & 3,713 & 4,527 \\
\hline
\end{tabular}

Average Percent Tree Cover by Province, and Areal Extent of Tree Cover Classes as a Percent of the Total Agricultural Area (\%)

\begin{tabular}{|c|c|c|c|c|c|c|c|c|c|c|c|c|c|c|c|c|}
\hline \multicolumn{2}{|l|}{ Australia } & \multirow{3}{*}{\multicolumn{3}{|c|}{$\begin{array}{c}\text { Average Tree Cover by Province } \\
\text { Percent Tree Cover } \\
(\%)\end{array}$}} & \multicolumn{12}{|c|}{ Areal Extent of Tree Cover Class as a Percent of Total Agricultural Area (\%) } \\
\hline \multirow{3}{*}{ State } & \multirow{3}{*}{ Area_km } & & & & \multirow{2}{*}{\multicolumn{4}{|c|}{$\begin{array}{c}\text { Year } 2000 \\
\text { Percent of Total Agrciultural Area (\%) }\end{array}$}} & & & 2010 & & & Change ( & 0 to 2010 & \\
\hline & & & & & & & & & \multicolumn{4}{|c|}{ Percent of Total Agrciultural Area (\%) } & \multicolumn{4}{|c|}{ Percent of Total Agrciultural Area (\%) } \\
\hline & & 2000 & 2010 & Change & $<10 \%$ & $10-20 \%$ & $20-30 \%$ & $>30 \%$ & $<10 \%$ & $10-20 \%$ & $20-30 \%$ & $>30 \%$ & $<10 \%$ & $10-20 \%$ & $20-30 \%$ & $>30 \%$ \\
\hline Australian Capital Territory & 61 & 5.80 & 7.13 & 1.33 & 91.80 & 6.56 & 1.64 & 0.00 & 91.80 & 6.56 & 1.64 & 0.00 & 0.00 & 0.00 & 0.00 & 0.00 \\
\hline New South Wales & 185,128 & 3.79 & 4.55 & 0.77 & 96.45 & 3.01 & 0.39 & 0.15 & 91.31 & 7.64 & 0.77 & 0.28 & -5.14 & 4.63 & 0.38 & 0.13 \\
\hline Northern Territory & 29,548 & 3.55 & 1.70 & -1.85 & 99.86 & 0.14 & 0.00 & 0.00 & 100.00 & 0.00 & 0.00 & 0.00 & 0.14 & -0.14 & 0.00 & 0.00 \\
\hline Queensland & 22,223 & 2.51 & 3.25 & 0.74 & 99.04 & 0.47 & 0.18 & 0.31 & 99.11 & 0.42 & 0.20 & 0.27 & 0.07 & -0.05 & 0.02 & -0.04 \\
\hline South Australia & 99,836 & 4.49 & 5.18 & 0.69 & 90.77 & 7.57 & 1.20 & 0.46 & 88.23 & 8.54 & 2.22 & 1.01 & -2.53 & 0.97 & 1.01 & 0.55 \\
\hline Tasmania & 4,742 & 18.42 & 21.69 & 3.28 & 24.65 & 45.85 & 13.41 & 16.09 & 13.05 & 42.83 & 21.00 & 23.11 & -11.60 & -3.02 & 7.59 & 7.02 \\
\hline Victoria & 108,917 & 8.32 & 9.92 & 1.60 & 72.50 & 17.87 & 6.09 & 3.54 & 67.58 & 18.87 & 7.25 & 6.30 & -4.92 & 1.00 & 1.15 & 2.76 \\
\hline Western Australia & 147,418 & 3.05 & 3.37 & 0.31 & 94.57 & 4.36 & 0.88 & 0.20 & 94.37 & 4.03 & 1.13 & 0.47 & -0.19 & -0.33 & 0.26 & 0.27 \\
\hline Australia - All & 597,873 & 4.61 & 5.29 & 0.68 & 90.37 & 6.92 & 1.76 & 0.96 & 87.33 & 8.58 & 2.38 & 1.71 & -3.04 & 1.66 & 0.62 & 0.76 \\
\hline
\end{tabular}


Asia Pacific Region : Tree Cover on Agricultural Land (2010)

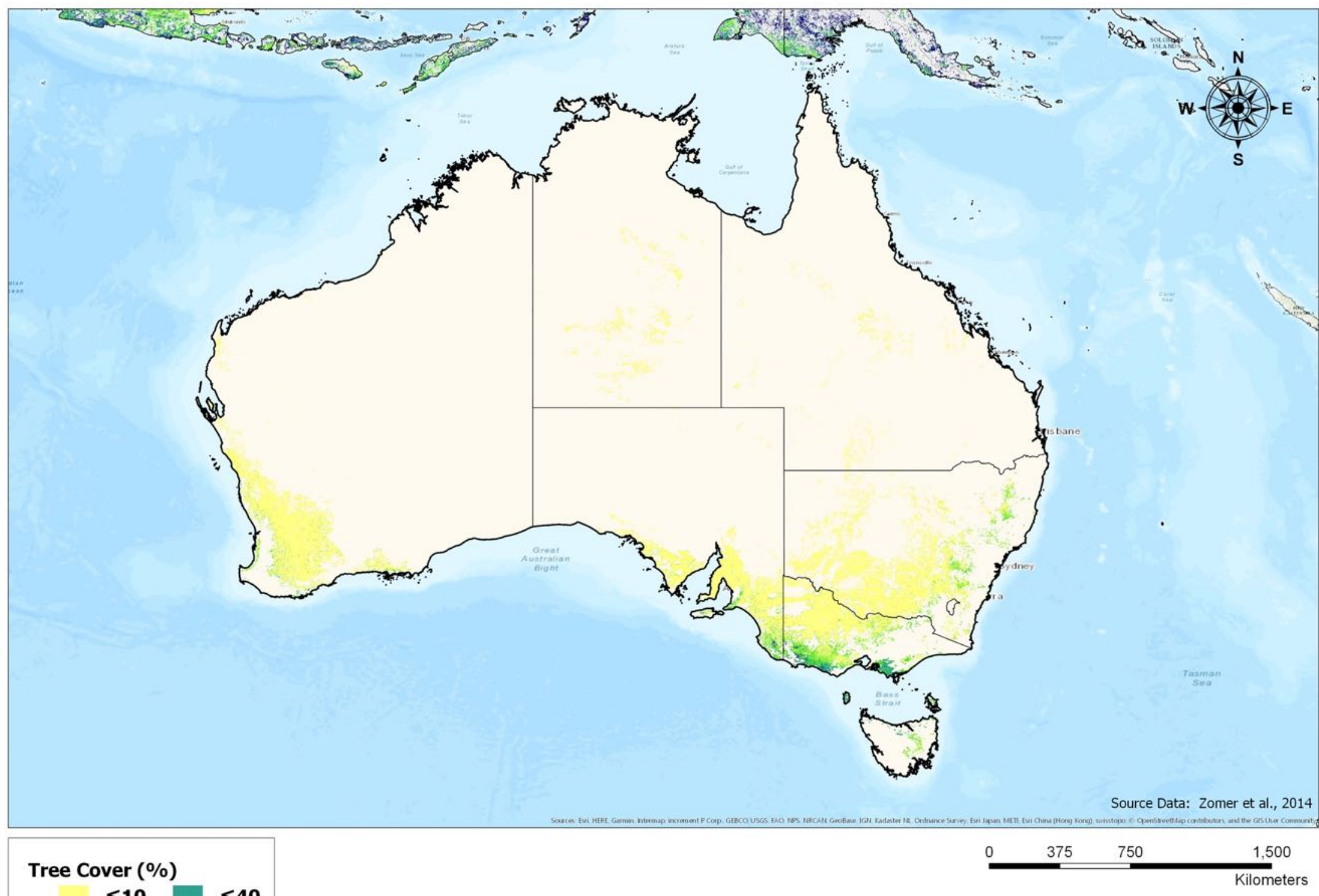

Australia 
Asia Pacific Region : Change in Tree Cover on Agricultural Land (2000 to 2010)

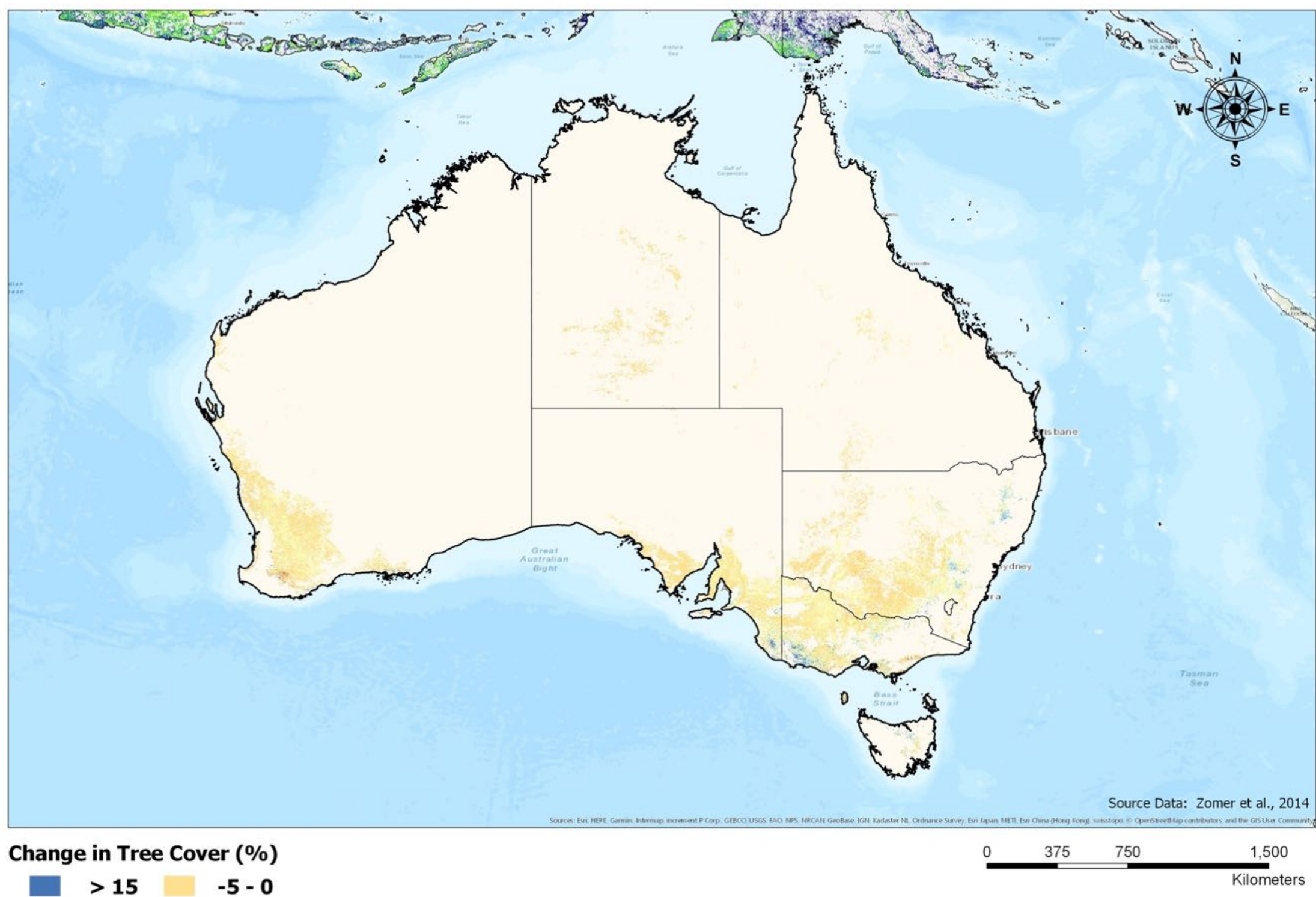

$10-15 \quad-10--5$

$5-10 \quad-15--10$

$0-5 \square \leq-15$

\section{Australia}


Average Percent Tree Cover by Province, and Areal Extent of Tree Cover Classes $\left(\mathrm{km}^{2}\right)$

\begin{tabular}{|c|c|c|c|c|c|c|c|c|c|c|c|c|c|c|c|c|}
\hline \multicolumn{2}{|c|}{ Bangladesh } & & \multicolumn{12}{|c|}{ Areal Extent of Tree Cover Class $\left(\mathrm{km}^{2}\right)$} \\
\hline \multirow{3}{*}{ Province } & \multirow{3}{*}{ Area_km } & & & & \multirow{2}{*}{\multicolumn{4}{|c|}{ Year 2000}} & \multirow{2}{*}{\multicolumn{4}{|c|}{ Year 2010}} & \multirow{2}{*}{\multicolumn{4}{|c|}{ Conge (2000 to 2010) }} \\
\hline & & \multicolumn{3}{|c|}{\begin{tabular}{|c|} 
Mean Tree Cover Percent by Province \\
$(\%)$
\end{tabular}} & & & & & & & ver Class ( & & & & & \\
\hline & & 2000 & 2010 & Change & $<10 \%$ & $10-20 \%$ & $20-30 \%$ & $>30 \%$ & $<10 \%$ & $10-20 \%$ & $20-30 \%$ & $>30 \%$ & $<10 \%$ & $10-20 \%$ & $20-30 \%$ & $>30 \%$ \\
\hline Barisal & 7,547 & 12.16 & 19.19 & 7.03 & 3,913 & 2,406 & 1,015 & 213 & 542 & 3,921 & 2,777 & 307 & $-3,371$ & 1,515 & 1,762 & 94 \\
\hline Chittagong & 12,738 & 24.06 & 24.23 & 0.17 & 2,930 & 4,217 & 1,803 & 3,788 & 1,192 & 5,078 & 3,021 & 3,447 & $-1,738$ & 861 & 1,218 & -341 \\
\hline Dhaka & 25,635 & 11.86 & 15.83 & 3.97 & 11,506 & 12,215 & 1,575 & 339 & 4,437 & 16,668 & 4,383 & 147 & $-7,069$ & 4,453 & 2,808 & -192 \\
\hline Khulna & 14,567 & 11.86 & 14.69 & 2.84 & 6,372 & 7,245 & 824 & 126 & 2,580 & 10,331 & 1,590 & 66 & $-3,792$ & 3,086 & 766 & -60 \\
\hline Rajshahi & 16,252 & 8.33 & 11.12 & 2.78 & 11,412 & 4,665 & 173 & 2 & 7,045 & 8,749 & 458 & 0 & $-4,367$ & 4,084 & 285 & -2 \\
\hline Rangpur & 15,154 & 8.20 & 13.87 & 5.67 & 10,981 & 4,053 & 120 & 0 & 3,485 & 10,410 & 1,259 & 0 & $-7,496$ & 6,357 & 1,139 & 0 \\
\hline Sylhet & 9,550 & 10.33 & 14.18 & 3.85 & 6,383 & 2,566 & 335 & 266 & 2,862 & 5,661 & 780 & 247 & $-3,521$ & 3,095 & 445 & -19 \\
\hline Bangladesh - All & 101,441 & 12.16 & 15.77 & 3.61 & 53,495 & 37,367 & 5,845 & 4,734 & 22,143 & 60,816 & 14,268 & 4,214 & $-31,352$ & 23,449 & 8,423 & -520 \\
\hline
\end{tabular}

Average Percent Tree Cover by Province, and Areal Extent of Tree Cover Classes as a Percent of the Total Agricultural Area (\%)

\begin{tabular}{|c|c|c|c|c|c|c|c|c|c|c|c|c|c|c|c|c|}
\hline \multicolumn{2}{|c|}{ Bangladesh } & \multirow{3}{*}{\multicolumn{3}{|c|}{$\begin{array}{l}\text { Average Tree Cover by Province } \\
\text { Percent Tree Cover } \\
(\%)\end{array}$}} & \multicolumn{12}{|c|}{ Areal Extent of Tree Cover Class as a Percent of Total Agricultural Area (\%) } \\
\hline \multirow{3}{*}{ Province } & \multirow{3}{*}{ Area_km } & & & & \multirow{2}{*}{\multicolumn{4}{|c|}{$\begin{array}{c}\text { Year } 2000 \\
\text { Percent of Total Agrciultural Area ( \% ) }\end{array}$}} & \multirow{2}{*}{\multicolumn{4}{|c|}{$\begin{array}{c}\text { Year } 2010 \\
\text { Percent of Total Agrciultural Area (\%) }\end{array}$}} & \multirow{2}{*}{\multicolumn{4}{|c|}{ Percent of Total Agrciultural Area (\%) }} \\
\hline & & & & & & & & & & & & & & & & \\
\hline & & 2000 & 2010 & Change & $<10 \%$ & $10-20 \%$ & $20-30 \%$ & $>30 \%$ & $<10 \%$ & $10-20 \%$ & $20-30 \%$ & $>30 \%$ & $<10 \%$ & $10-20 \%$ & $20-30 \%$ & $>30 \%$ \\
\hline Barisal & 7,547 & 12.16 & 19.19 & 7.03 & 51.85 & 31.88 & 13.45 & 2.82 & 7.18 & 51.95 & 36.80 & 4.07 & -44.67 & 20.07 & 23.35 & 1.25 \\
\hline Chittagong & 12,738 & 24.06 & 24.23 & 0.17 & 23.00 & 33.11 & 14.15 & 29.74 & 9.36 & 39.87 & 23.72 & 27.06 & -13.64 & 6.76 & 9.56 & -2.68 \\
\hline Dhaka & 25,635 & 11.86 & 15.83 & 3.97 & 44.88 & 47.65 & 6.14 & 1.32 & 17.31 & 65.02 & 17.10 & 0.57 & -27.58 & 17.37 & 10.95 & -0.75 \\
\hline Khulna & 14,567 & 11.86 & 14.69 & 2.84 & 43.74 & 49.74 & 5.66 & 0.86 & 17.71 & 70.92 & 10.92 & 0.45 & -26.03 & 21.19 & 5.26 & -0.41 \\
\hline Rajshahi & 16,252 & 8.33 & 11.12 & 2.78 & 70.22 & 28.70 & 1.06 & 0.01 & 43.35 & 53.83 & 2.82 & 0.00 & -26.87 & 25.13 & 1.75 & -0.01 \\
\hline Rangpur & 15,154 & 8.20 & 13.87 & 5.67 & 72.46 & 26.75 & 0.79 & 0.00 & 23.00 & 68.70 & 8.31 & 0.00 & -49.47 & 41.95 & 7.52 & 0.00 \\
\hline Sylhet & 9,550 & 10.33 & 14.18 & 3.85 & 66.84 & 26.87 & 3.51 & 2.79 & 29.97 & 59.28 & 8.17 & 2.59 & -36.87 & 32.41 & 4.66 & -0.20 \\
\hline Bangladesh - All & 101,441 & 12.16 & 15.77 & 3.61 & 52.74 & 36.84 & 5.76 & 4.67 & 21.83 & 59.95 & 14.07 & 4.15 & -30.91 & 23.12 & 8.30 & -0.51 \\
\hline
\end{tabular}


Asia Pacific Region : Tree Cover on Agricultural Land (2010)

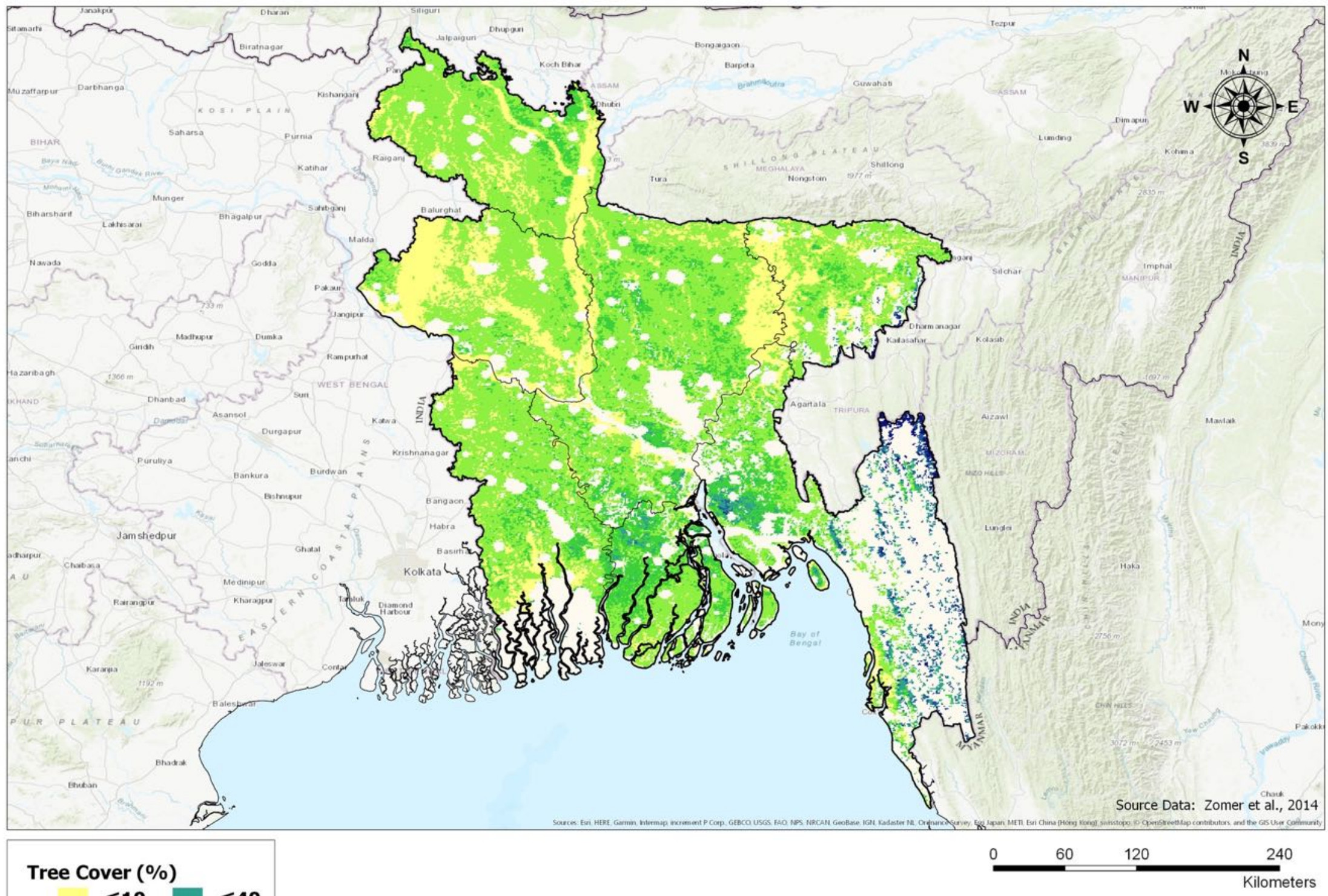
$\leq \mathbf{1 0}$
$\leq 40$
$\leq \mathbf{2 0}$

Bangladesh 
Asia Pacific Region : Change in Tree Cover on Agricultural Land (2000 - 2010)

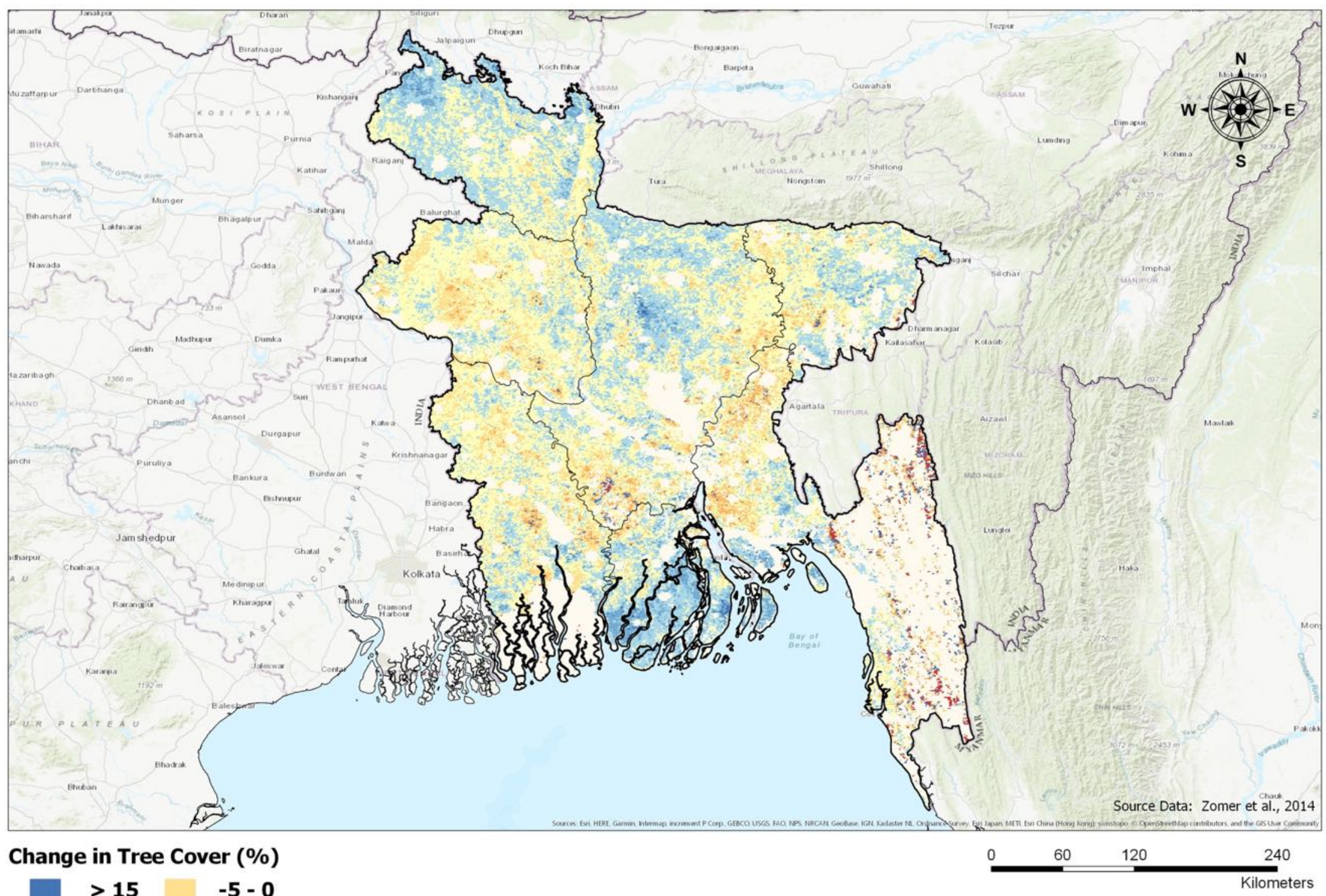

\begin{tabular}{|r|r|}
\hline$>15$ & $-5-0$ \\
$10-15$ & $-10--5$ \\
$5-10$ & $-15--10$ \\
$0-5$ & $\leq-15$
\end{tabular}

Bangladesh 
Appendix 4: Bhutan

Average Percent Tree Cover by Province, and Areal Extent of Tree Cover Classes $\left(\mathrm{km}^{2}\right)$

\begin{tabular}{|c|c|c|c|c|c|c|c|c|c|c|c|c|c|c|c|c|}
\hline \multicolumn{2}{|c|}{ Bhutan } & \multirow{3}{*}{\multicolumn{3}{|c|}{\begin{tabular}{|c|} 
Average Tree Cover by Province \\
Mean Tree Cover Percent by Province \\
$(\%)$ \\
\end{tabular}}} & \multicolumn{12}{|c|}{ Areal Extent of Tree Cover Class $\left(\mathrm{km}^{2}\right)$} \\
\hline \multirow{3}{*}{ Province } & \multirow{3}{*}{ Area_km } & & & & \multicolumn{4}{|c|}{ Year 2000} & \multirow{2}{*}{\multicolumn{4}{|c|}{$\begin{array}{c}\text { Year } 2010 \\
\text { Area by Tree Cover Class }\left(\mathrm{km}^{2}\right)\end{array}$}} & \multirow{2}{*}{\multicolumn{4}{|c|}{ Change (2000 to 2010) }} \\
\hline & & & & & & ea by Tree $\mathrm{C}$ & ver Class ( $k$ & & & & & & & & over Class & \\
\hline & & 2000 & 2010 & Change & $<10 \%$ & $10-20 \%$ & $20-30 \%$ & $>30 \%$ & $<10 \%$ & $10-20 \%$ & $20-30 \%$ & $>30 \%$ & $<10 \%$ & $10-20 \%$ & $20-30 \%$ & $>30 \%$ \\
\hline Bumthang & 398 & 45.28 & 49.87 & 4.59 & 17 & 33 & 51 & 297 & 11 & 20 & 38 & 329 & -6 & -13 & -13 & 32 \\
\hline Chhukha & 102 & 45.66 & 49.35 & 3.70 & 1 & 6 & 11 & 84 & 1 & 4 & 8 & 89 & 0 & -2 & -3 & 5 \\
\hline Dagana & 275 & 47.51 & 53.33 & 5.82 & 4 & 7 & 21 & 243 & 3 & 5 & 10 & 257 & -1 & -2 & -11 & 14 \\
\hline Gasa & 133 & 32.47 & 34.23 & 1.76 & 28 & 16 & 23 & 66 & 27 & 15 & 13 & 78 & -1 & -1 & -10 & 12 \\
\hline Haа & 47 & 15.98 & 18.26 & 2.28 & 28 & 7 & 3 & 9 & 27 & 7 & 2 & 11 & -1 & 0 & -1 & 2 \\
\hline Lhuentse & 834 & 53.28 & 54.65 & 1.37 & 11 & 36 & 65 & 722 & 16 & 24 & 42 & 752 & 5 & -12 & -23 & 30 \\
\hline Monggar & 337 & 46.53 & 52.23 & 5.71 & 4 & 20 & 57 & 256 & 0 & 7 & 26 & 304 & -4 & -13 & -31 & 48 \\
\hline Paro & 119 & 32.16 & 33.03 & 0.87 & 36 & 18 & 13 & 52 & 35 & 19 & 11 & 54 & -1 & 1 & -2 & 2 \\
\hline Pemagatshel & 301 & 56.18 & 62.10 & 5.92 & 0 & 3 & 13 & 285 & 0 & 1 & 9 & 291 & 0 & -2 & -4 & 6 \\
\hline Punakha & 246 & 53.56 & 54.64 & 1.08 & 2 & 17 & 12 & 215 & 2 & 6 & 23 & 215 & 0 & -11 & 11 & 0 \\
\hline Samdrupjongkhar & 703 & 56.55 & 59.88 & 3.33 & 1 & 13 & 35 & 654 & 4 & 12 & 35 & 652 & 3 & -1 & 0 & -2 \\
\hline Samtse & 159 & 39.40 & 41.91 & 2.50 & 10 & 18 & 24 & 107 & 5 & 12 & 30 & 112 & -5 & -6 & 6 & 5 \\
\hline Sarpang & 343 & 48.19 & 52.15 & 3.96 & 18 & 19 & 40 & 266 & 13 & 21 & 27 & 282 & -5 & 2 & -13 & 16 \\
\hline Thimphu & 83 & 26.60 & 28.40 & 1.80 & 41 & 9 & 1 & 32 & 37 & 9 & 5 & 32 & -4 & 0 & 4 & 0 \\
\hline Trashigang & 388 & 40.31 & 45.54 & 5.23 & 4 & 63 & 73 & 248 & 0 & 32 & 56 & 300 & -4 & -31 & -17 & 52 \\
\hline Trongsa & 364 & 48.29 & 52.12 & 3.83 & 6 & 21 & 36 & 301 & 9 & 5 & 21 & 329 & 3 & -16 & -15 & 28 \\
\hline Tsirang & 90 & 43.54 & 51.41 & 7.87 & 0 & 6 & 18 & 66 & 0 & 1 & 10 & 79 & 0 & -5 & -8 & 13 \\
\hline Wangduephodrang & 615 & 41.46 & 44.19 & 2.73 & 50 & 82 & 72 & 411 & 49 & 48 & 66 & 452 & -1 & -34 & -6 & 41 \\
\hline Yangtse & 570 & 49.29 & 53.18 & 3.89 & 20 & 35 & 62 & 453 & 14 & 29 & 28 & 499 & -6 & -6 & -34 & 46 \\
\hline Zhemgang & 377 & 61.04 & 66.02 & 4.98 & 0 & 6 & 6 & 365 & 1 & 5 & 0 & 371 & 1 & -1 & -6 & 6 \\
\hline Bhutan - All & 6,485 & 48.32 & 51.95 & 3.63 & 281 & 435 & 637 & 5,132 & 254 & 282 & 460 & 5,489 & -27 & -153 & -177 & 357 \\
\hline
\end{tabular}


Average Percent Tree Cover by Province, and Areal Extent of Tree Cover Classes as a Percent of the Total Agricultural Area (\%)

\begin{tabular}{|c|c|c|c|c|c|c|c|c|c|c|c|c|c|c|c|c|}
\hline \multicolumn{2}{|c|}{ Bhutan } & \multirow{3}{*}{\multicolumn{3}{|c|}{$\begin{array}{c}\text { Average Tree Cover by Province } \\
\text { Percent Tree Cover } \\
(\%)\end{array}$}} & \multicolumn{12}{|c|}{ Areal Extent of Tree Cover Class as a Percent of Total Agricultural Area (\%) } \\
\hline \multirow{3}{*}{ Province } & \multirow{3}{*}{ Area_km } & & & & \multirow{2}{*}{\multicolumn{4}{|c|}{$\begin{array}{c}\text { Year } 2000 \\
\text { Percent of Total Agrciultural Area (\%) }\end{array}$}} & & Yeal & & & & Change (2 & 0 to 2010) & \\
\hline & & & & & & & & & \multicolumn{4}{|c|}{ Percent of Total Agrciultural Area (\%) } & \multicolumn{4}{|c|}{ Percent of Total Agrciultural Area (\%) } \\
\hline & & 2000 & 2010 & Change & $<10 \%$ & $10-20 \%$ & $20-30 \%$ & $>30 \%$ & $<10 \%$ & $10-20 \%$ & $20-30 \%$ & $>30 \%$ & $<10 \%$ & $10-20 \%$ & $20-30 \%$ & $>30 \%$ \\
\hline Bumthang & 398 & 45.28 & 49.87 & 4.59 & 4.27 & 8.29 & 12.81 & 74.62 & 2.76 & 5.03 & 9.55 & 82.66 & -1.51 & -3.27 & -3.27 & 8.04 \\
\hline Chhukha & 102 & 45.66 & 49.35 & 3.70 & 0.98 & 5.88 & 10.78 & 82.35 & 0.98 & 3.92 & 7.84 & 87.26 & 0.00 & -1.96 & -2.94 & 4.90 \\
\hline Dagana & 275 & 47.51 & 53.33 & 5.82 & 1.45 & 2.55 & 7.64 & 88.36 & 1.09 & 1.82 & 3.64 & 93.46 & -0.36 & -0.73 & -4.00 & 5.09 \\
\hline Gasa & 133 & 32.47 & 34.23 & 1.76 & 21.05 & 12.03 & 17.29 & 49.62 & 20.30 & 11.28 & 9.77 & 58.65 & -0.75 & -0.75 & -7.52 & 9.02 \\
\hline Haa & 47 & 15.98 & 18.26 & 2.28 & 59.57 & 14.89 & 6.38 & 19.15 & 57.45 & 14.89 & 4.26 & 23.40 & -2.13 & 0.00 & -2.13 & 4.26 \\
\hline Lhuentse & 834 & 53.28 & 54.65 & 1.37 & 1.32 & 4.32 & 7.79 & 86.57 & 1.92 & 2.88 & 5.04 & 90.17 & 0.60 & -1.44 & -2.76 & 3.60 \\
\hline Monggar & 337 & 46.53 & 52.23 & 5.71 & 1.19 & 5.93 & 16.91 & 75.96 & 0.00 & 2.08 & 7.72 & 90.21 & -1.19 & -3.86 & -9.20 & 14.24 \\
\hline Paro & 119 & 32.16 & 33.03 & 0.87 & 30.25 & 15.13 & 10.92 & 43.70 & 29.41 & 15.97 & 9.24 & 45.38 & -0.84 & 0.84 & -1.68 & 1.68 \\
\hline Pemagatshel & 301 & 56.18 & 62.10 & 5.92 & 0.00 & 1.00 & 4.32 & 94.69 & 0.00 & 0.33 & 2.99 & 96.68 & 0.00 & -0.66 & -1.33 & 1.99 \\
\hline Punakha & 246 & 53.56 & 54.64 & 1.08 & 0.81 & 6.91 & 4.88 & 87.40 & 0.81 & 2.44 & 9.35 & 87.40 & 0.00 & -4.47 & 4.47 & 0.00 \\
\hline Samdrupjongkhar & 703 & 56.55 & 59.88 & 3.33 & 0.14 & 1.85 & 4.98 & 93.03 & 0.57 & 1.71 & 4.98 & 92.75 & 0.43 & -0.14 & 0.00 & -0.28 \\
\hline Samtse & 159 & 39.40 & 41.91 & 2.50 & 6.29 & 11.32 & 15.09 & 67.30 & 3.14 & 7.55 & 18.87 & 70.44 & -3.14 & -3.77 & 3.77 & 3.14 \\
\hline Sarpang & 343 & 48.19 & 52.15 & 3.96 & 5.25 & 5.54 & 11.66 & 77.55 & 3.79 & 6.12 & 7.87 & 82.22 & -1.46 & 0.58 & -3.79 & 4.66 \\
\hline Thimphu & 83 & 26.60 & 28.40 & 1.80 & 49.40 & 10.84 & 1.20 & 38.55 & 44.58 & 10.84 & 6.02 & 38.55 & -4.82 & 0.00 & 4.82 & 0.00 \\
\hline Trashigang & 388 & 40.31 & 45.54 & 5.23 & 1.03 & 16.24 & 18.81 & 63.92 & 0.00 & 8.25 & 14.43 & 77.32 & -1.03 & -7.99 & $\begin{array}{l}-4.38 \\
-4.32\end{array}$ & 13.40 \\
\hline Trongsa & 364 & 48.29 & 52.12 & 3.83 & 1.65 & 5.77 & 9.89 & 82.69 & 2.47 & 1.37 & 5.77 & 90.39 & 0.82 & -4.40 & -4.12 & 7.69 \\
\hline Tsirang & 90 & 43.54 & 51.41 & 7.87 & 0.00 & 6.67 & 20.00 & 73.33 & 0.00 & 1.11 & 11.11 & 87.78 & 0.00 & -5.56 & -8.89 & 14.44 \\
\hline Wangduephodrang & 615 & 41.46 & 44.19 & $\begin{array}{l}2.01 \\
2.73\end{array}$ & 8.13 & $\begin{array}{l}0.01 \\
13.33\end{array}$ & 11.71 & 66.83 & 7.97 & 7.80 & 10.73 & 73.50 & $\begin{array}{l}-0.16 \\
-0.04\end{array}$ & $\begin{array}{l}-5.50 \\
-5.53\end{array}$ & $\begin{array}{l}-0.05 \\
-0.98\end{array}$ & $\begin{array}{r}\begin{array}{r}7.44 \\
6.67\end{array} \\
\end{array}$ \\
\hline Yangtse & 570 & 49.29 & 53.18 & 3.89 & 3.51 & 6.14 & 10.88 & 79.47 & 2.46 & 5.09 & 4.91 & 87.54 & -1.05 & -1.05 & -5.96 & 8.07 \\
\hline Zhemgang & 377 & 61.04 & 66.02 & 4.98 & 0.00 & 1.59 & 1.59 & 96.82 & 0.27 & 1.33 & 0.00 & 98.41 & 0.27 & -0.27 & -1.59 & 1.59 \\
\hline Bhutan - All & 6.485 & 48.32 & 51.95 & 3.63 & 4.33 & 6.71 & 9.82 & 79.14 & 3.92 & 4.35 & 7.09 & 84.64 & -0.42 & -2.36 & -2.73 & 5.51 \\
\hline
\end{tabular}


Asia Pacific Region : Tree Cover on Agricultural Land (2010)

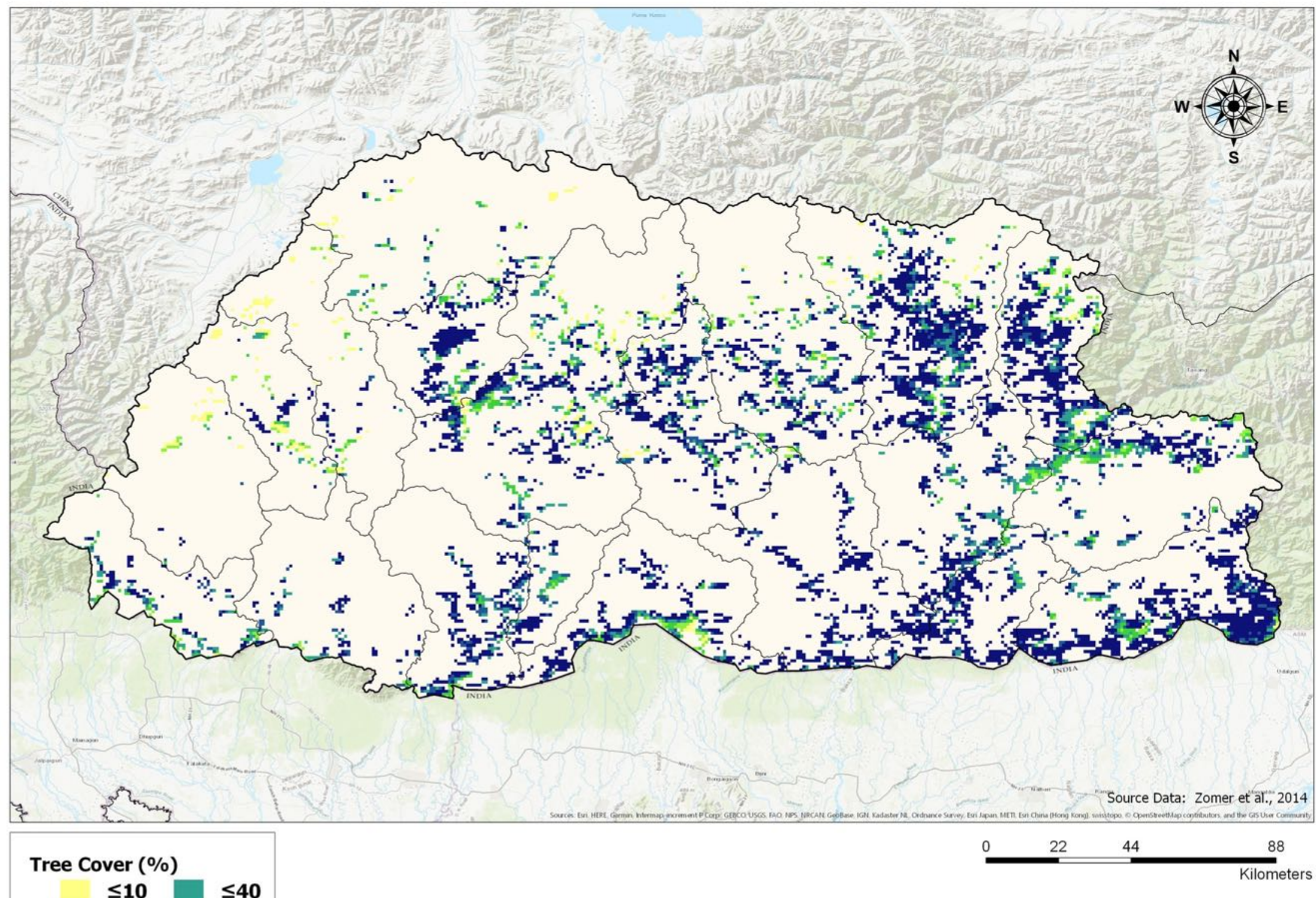

Bhutan 
Asia Pacific Region : Change in Tree Cover on Agricultural Land (2000 - 2010)

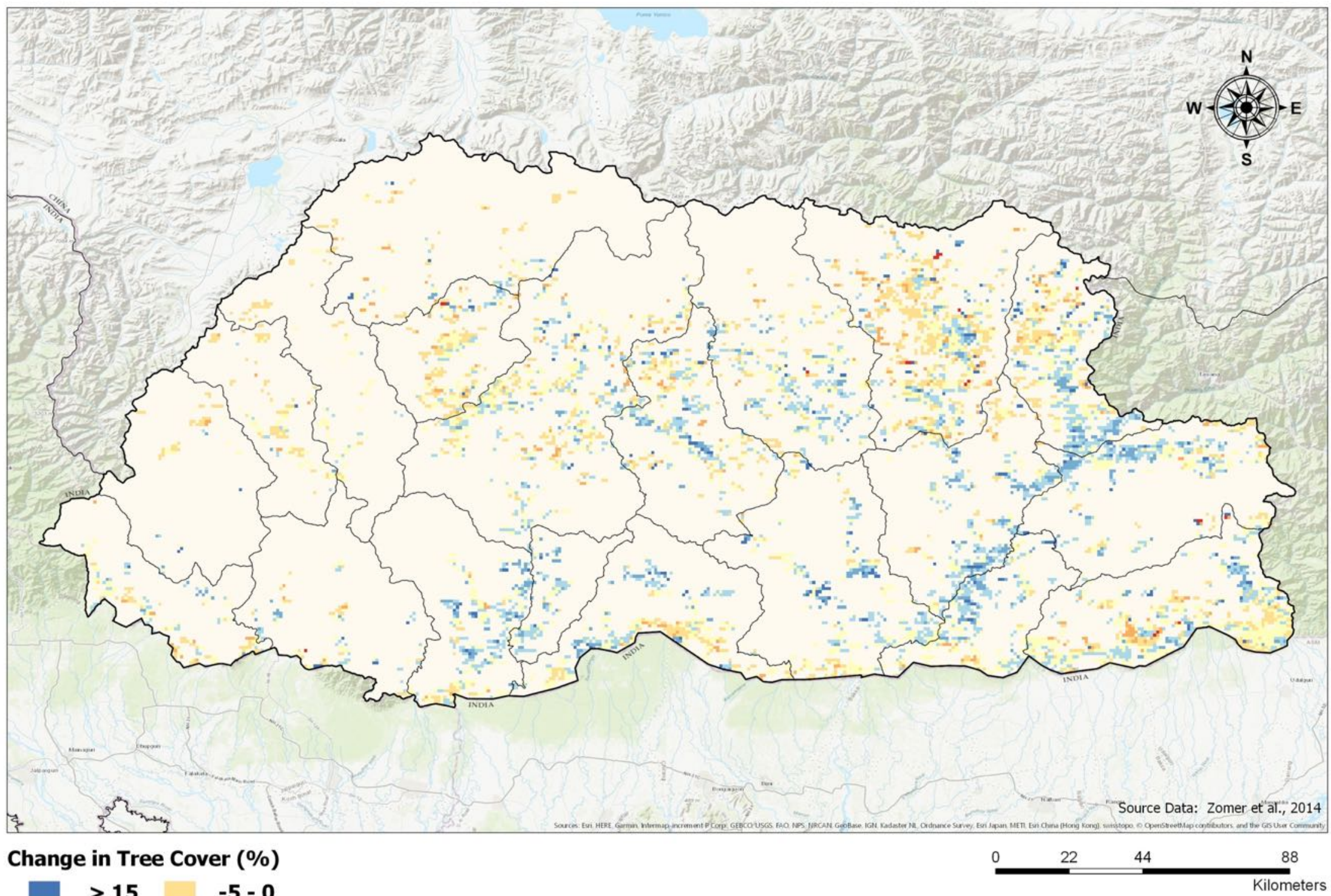
$>15 \quad-5-0$
$\begin{aligned} 10-15 & -10--5 \\ 5-10 & -15--10\end{aligned}$
$0-5 \square \leq-15$

\section{Bhutan}




\section{Appendix 5: Cambodia}

Average Percent Tree Cover by Province, and Areal Extent of Tree Cover Classes $\left(\mathrm{km}^{2}\right)$

\begin{tabular}{|c|c|c|c|c|c|c|c|c|c|c|c|c|c|c|c|c|}
\hline \multicolumn{2}{|l|}{ Cambodia } & \multirow{3}{*}{\multicolumn{3}{|c|}{\begin{tabular}{|c|} 
Average Tree Cover by Province \\
Mean Tree Cover Percent by Province \\
$(\%)$
\end{tabular}}} & \multicolumn{12}{|c|}{ Areal Extent of Tree Cover Class $\left(\mathrm{km}^{2}\right)$} \\
\hline \multirow{3}{*}{ Province } & \multirow{3}{*}{ Area_km } & & & & \multicolumn{4}{|c|}{ Year 2000} & \multirow{2}{*}{\multicolumn{4}{|c|}{$\begin{array}{c}\text { Year } 2010 \\
\end{array}$}} & \multirow{2}{*}{\multicolumn{4}{|c|}{$\begin{array}{l}\text { Change (2000 to 2010) } \\
\text { Area } \text { Tree Cover Class }\left(\mathrm{km}^{2}\right)\end{array}$}} \\
\hline & & & & & & by Tree $\mathrm{C}$ & ver Class (k & & & & & & & & & \\
\hline & & 2000 & 2010 & Change & $<10 \%$ & $10-20 \%$ & $20-30 \%$ & $>\mathbf{3 0} \%$ & $<10 \%$ & $10-20 \%$ & $20-30 \%$ & $>30 \%$ & $<10 \%$ & $10-20 \%$ & $20-30 \%$ & $>30 \%$ \\
\hline Bântéay Méanchey & 3,898 & 8.23 & 7.95 & -0.27 & 2,827 & 773 & 228 & 70 & 2,815 & 975 & 86 & 22 & -12 & 202 & -142 & -48 \\
\hline Batdâmbâng & 4,067 & 16.03 & 12.77 & -3.26 & 2,483 & 541 & 233 & 810 & 2,301 & 1,130 & 235 & 401 & -182 & 589 & 2 & -409 \\
\hline Kâmpóng Cham & 3,152 & 11.02 & 11.05 & 0.03 & 2,077 & 602 & 189 & 284 & 1,917 & 823 & 308 & 104 & -160 & 221 & 119 & -180 \\
\hline Kâmpóng Chhnang & 2,317 & 11.31 & 11.64 & 0.33 & 1,509 & 429 & 190 & 189 & 1,511 & 446 & 184 & 176 & 2 & 17 & -6 & -13 \\
\hline Kâmpóng Spœ & 3,516 & 13.14 & 11.53 & -1.62 & 2,315 & 330 & 254 & 617 & 2,412 & 389 & 264 & 451 & 97 & 59 & 10 & -166 \\
\hline Kâmpóng Thum & 3,166 & 12.25 & 12.11 & -0.15 & 1,820 & 725 & 335 & 286 & 1,916 & 693 & 333 & 224 & 96 & -32 & -2 & -62 \\
\hline Kâmpôt & 2,310 & 11.72 & 10.91 & -0.82 & 1,608 & 307 & 132 & 263 & 1,663 & 346 & 118 & 183 & 55 & 39 & -14 & -80 \\
\hline Kândal & 2,851 & 10.50 & 11.92 & 1.42 & 1,950 & 471 & 207 & 223 & 1,619 & 788 & 243 & 201 & -331 & 317 & 36 & -22 \\
\hline Kaôh Kong & 397 & 31.44 & 26.32 & -5.12 & 40 & 73 & 96 & 188 & 58 & 104 & 96 & 139 & 18 & 31 & 0 & -49 \\
\hline Kep & 109 & 12.89 & 12.77 & -0.12 & 73 & 16 & 6 & 14 & 72 & 20 & 8 & 9 & -1 & 4 & 2 & -5 \\
\hline Krâchéh & 2,838 & 23.97 & 20.07 & -3.90 & 699 & 616 & 631 & 892 & 659 & 927 & 784 & 468 & -40 & 311 & 153 & -424 \\
\hline Krong Pailin & 143 & 51.63 & 17.68 & -33.95 & 2 & 8 & 17 & 116 & 63 & 48 & 6 & 26 & 61 & 40 & -11 & -90 \\
\hline Krong Preah Sihanouk & 252 & 27.50 & 26.66 & -0.84 & 36 & 75 & 63 & 78 & 39 & 63 & 64 & 86 & 3 & -12 & 1 & 8 \\
\hline Môndól Kiri & 1,562 & 37.30 & 37.58 & 0.28 & 68 & 115 & 244 & 1,135 & 31 & 128 & 231 & 1,172 & -37 & 13 & -13 & 37 \\
\hline Otdar Mean Chey & 682 & 23.38 & 17.12 & -6.26 & 238 & 85 & 103 & 256 & 270 & 155 & 151 & 106 & 32 & 70 & 48 & -150 \\
\hline Phnom Penh & 170 & 5.87 & 5.02 & -0.85 & 149 & 13 & 4 & 4 & 155 & 12 & 3 & 0 & 6 & -1 & -1 & -4 \\
\hline Pouthisat & 2,453 & 16.58 & 14.98 & -1.61 & 1,407 & 288 & 169 & 589 & 1,511 & 244 & 172 & 526 & 104 & -44 & 3 & -63 \\
\hline Preah Vihéar & 1,390 & 30.15 & 29.49 & -0.66 & 188 & 190 & 402 & 610 & 146 & 246 & 414 & 584 & -42 & 56 & 12 & -26 \\
\hline Prey Vêng & 4,608 & 4.36 & 5.81 & 1.45 & 4,400 & 179 & 21 & 8 & 4,251 & 340 & 15 & 2 & -149 & 161 & -6 & -6 \\
\hline Rôtânôkiri & 1,820 & 43.42 & 39.47 & -3.95 & 9 & 44 & 208 & 1,559 & 20 & 135 & 363 & 1,302 & 11 & 91 & 155 & -257 \\
\hline Siemréab & 3,908 & 13.75 & 11.43 & -2.32 & 2,229 & 482 & 569 & 628 & 2,414 & 685 & 493 & 316 & 185 & 203 & -76 & -312 \\
\hline Stœng Trêng & 1,044 & 37.93 & 36.38 & -1.55 & 106 & 70 & 198 & 670 & 89 & 119 & 203 & 633 & -17 & 49 & 5 & -37 \\
\hline Svay Rieng & 2,429 & 8.19 & 7.87 & -0.32 & 1,864 & 419 & 83 & 63 & 1,975 & 383 & 57 & 14 & 111 & -36 & -26 & -49 \\
\hline Takêv & 3,348 & 5.41 & 6.24 & 0.83 & 3,002 & 212 & 73 & 61 & 2,934 & 250 & 95 & 69 & -68 & 38 & 22 & 8 \\
\hline Tbong Khmum & 3,382 & 21.36 & 18.31 & -3.06 & 978 & 857 & 685 & 862 & 994 & 1,212 & 695 & 481 & 16 & 355 & 10 & -381 \\
\hline Cambodia - All & 55,811 & 15.35 & 14.21 & -1.15 & 32,077 & 7,919 & 5,340 & 10,475 & 31,835 & 10,660 & 5,621 & 7,695 & -242 & 2,741 & 281 & $-2,780$ \\
\hline
\end{tabular}


Average Percent Tree Cover by Province, and Areal Extent of Tree Cover Classes as a Percent of the Total Agricultural Area (\%)

\begin{tabular}{|c|c|c|c|c|c|c|c|c|c|c|c|c|c|c|c|c|}
\hline \multicolumn{2}{|l|}{ Cambodia } & \multirow{3}{*}{\multicolumn{3}{|c|}{$\begin{array}{c}\text { Average Tree Cover by Province } \\
\text { Percent Tree Cover } \\
(\%)\end{array}$}} & \multicolumn{12}{|c|}{ Areal Extent of Tree Cover Class as a Percent of Total Agricultural Area (\%) } \\
\hline \multirow{3}{*}{ Province } & \multirow{3}{*}{ Area_km } & & & & \multirow{2}{*}{\multicolumn{4}{|c|}{$\begin{array}{c}\text { Year } 2000 \\
\text { Percent of Total Agrciultural Area }(\%)\end{array}$}} & \multirow{2}{*}{\multicolumn{4}{|c|}{$\begin{array}{c}\text { Year 2010 } \\
\text { Percent of Total Agrciultural Area (\%) }\end{array}$}} & \multirow{2}{*}{\multicolumn{4}{|c|}{ Percent of Total Agrciultural Area (\%) }} \\
\hline & & & & & & & & & & & & & & & & \\
\hline & & 2000 & 2010 & Change & $<10 \%$ & $10-20 \%$ & $20-30 \%$ & $>30 \%$ & $<10 \%$ & $10-20 \%$ & $20-30 \%$ & $>30 \%$ & $<10 \%$ & $10-20 \%$ & $20-30 \%$ & $>30 \%$ \\
\hline Bântéay Méanchey & 3,898 & 8.23 & 7.95 & -0.27 & 72.52 & 19.83 & 5.85 & 1.80 & 72.22 & 25.01 & 2.21 & 0.56 & -0.31 & 5.18 & -3.64 & -1.23 \\
\hline Batdâmbâng & 4,067 & 16.03 & 12.77 & -3.26 & 61.05 & 13.30 & 5.73 & 19.92 & 56.58 & 27.78 & 5.78 & 9.86 & -4.48 & 14.48 & 0.05 & -10.06 \\
\hline Kâmpóng Cham & 3,152 & 11.02 & 11.05 & 0.03 & 65.90 & 19.10 & 6.00 & 9.01 & 60.82 & 26.11 & 9.77 & 3.30 & -5.08 & 7.01 & 3.78 & -5.71 \\
\hline Kâmpóng Chhnang & 2,317 & 11.31 & 11.64 & 0.33 & 65.13 & 18.52 & 8.20 & 8.16 & 65.21 & 19.25 & 7.94 & 7.60 & 0.09 & 0.73 & -0.26 & -0.56 \\
\hline Kâmpóng Spœe & 3,516 & 13.14 & 11.53 & -1.62 & 65.84 & 9.39 & 7.22 & 17.55 & 68.60 & 11.06 & 7.51 & 12.83 & 2.76 & 1.68 & 0.28 & -4.72 \\
\hline Kâmpóng Thum & 3,166 & 12.25 & 12.11 & -0.15 & 57.49 & 22.90 & 10.58 & 9.03 & 60.52 & 21.89 & 10.52 & 7.08 & 3.03 & -1.01 & -0.06 & -1.96 \\
\hline Kâmpôt & 2,310 & 11.72 & 10.91 & -0.82 & 69.61 & 13.29 & 5.71 & 11.39 & 71.99 & 14.98 & 5.11 & 7.92 & 2.38 & 1.69 & -0.61 & -3.46 \\
\hline Kândal & 2,851 & 10.50 & 11.92 & 1.42 & 68.40 & 16.52 & 7.26 & 7.82 & 56.79 & 27.64 & 8.52 & 7.05 & -11.61 & 11.12 & 1.26 & -0.77 \\
\hline Kaôh Kong & 397 & 31.44 & 26.32 & -5.12 & 10.08 & 18.39 & 24.18 & 47.36 & 14.61 & 26.20 & 24.18 & 35.01 & 4.53 & 7.81 & 0.00 & -12.34 \\
\hline Kep & 109 & 12.89 & 12.77 & -0.12 & 66.97 & 14.68 & 5.50 & 12.84 & 66.06 & 18.35 & 7.34 & 8.26 & -0.92 & 3.67 & 1.83 & -4.59 \\
\hline Krâchéh & 2,838 & 23.97 & 20.07 & -3.90 & 24.63 & 21.71 & 22.23 & 31.43 & 23.22 & 32.66 & 27.63 & 16.49 & -1.41 & 10.96 & 5.39 & -14.94 \\
\hline Krong Pailin & 143 & 51.63 & 17.68 & -33.95 & 1.40 & 5.59 & 11.89 & 81.12 & 44.06 & 33.57 & 4.20 & 18.18 & 42.66 & 27.97 & -7.69 & -62.94 \\
\hline Krong Preah Sihanouk & 252 & 27.50 & 26.66 & -0.84 & 14.29 & 29.76 & 25.00 & 30.95 & 15.48 & 25.00 & 25.40 & 34.13 & 1.19 & -4.76 & 0.40 & 3.17 \\
\hline Môndól Kiri & 1,562 & 37.30 & 37.58 & 0.28 & 4.35 & 7.36 & 15.62 & 72.66 & 1.98 & 8.19 & 14.79 & 75.03 & -2.37 & 0.83 & -0.83 & 2.37 \\
\hline Otdar Mean Chey & 682 & 23.38 & 17.12 & -6.26 & 34.90 & 12.46 & 15.10 & 37.54 & 39.59 & 22.73 & 22.14 & 15.54 & 4.69 & 10.26 & 7.04 & -21.99 \\
\hline Phnom Penh & 170 & 5.87 & 5.02 & -0.85 & 87.65 & 7.65 & 2.35 & 2.35 & 91.18 & 7.06 & 1.76 & 0.00 & 3.53 & -0.59 & -0.59 & -2.35 \\
\hline Pouthisat & 2,453 & 16.58 & 14.98 & -1.61 & 57.36 & 11.74 & 6.89 & 24.01 & 61.60 & 9.95 & 7.01 & 21.44 & 4.24 & -1.79 & 0.12 & -2.57 \\
\hline Preah Vihéar & 1,390 & 30.15 & 29.49 & -0.66 & 13.53 & 13.67 & 28.92 & 43.89 & 10.50 & 17.70 & 29.78 & 42.01 & -3.02 & 4.03 & 0.86 & -1.87 \\
\hline Prey Vêng & 4,608 & 4.36 & 5.81 & 1.45 & 95.49 & 3.88 & 0.46 & 0.17 & 92.25 & 7.38 & 0.33 & 0.04 & -3.23 & 3.49 & -0.13 & -0.13 \\
\hline Rôtânôkiri & 1,820 & 43.42 & 39.47 & -3.95 & 0.49 & 2.42 & 11.43 & 85.66 & 1.10 & 7.42 & 19.95 & 71.54 & 0.60 & 5.00 & 8.52 & -14.12 \\
\hline Siemréab & 3,908 & 13.75 & 11.43 & -2.32 & 57.04 & 12.33 & 14.56 & 16.07 & 61.77 & 17.53 & 12.62 & 8.09 & 4.73 & 5.19 & -1.94 & -7.98 \\
\hline Stœng Trêng & 1,044 & 37.93 & 36.38 & -1.55 & 10.15 & 6.71 & 18.97 & 64.18 & 8.52 & 11.40 & 19.44 & 60.63 & -1.63 & 4.69 & 0.48 & -3.54 \\
\hline Svay Rieng & 2,429 & 8.19 & 7.87 & -0.32 & 76.74 & 17.25 & 3.42 & 2.59 & 81.31 & 15.77 & 2.35 & 0.58 & 4.57 & -1.48 & -1.07 & -2.02 \\
\hline Takêv & 3,348 & 5.41 & 6.24 & 0.83 & 89.67 & 6.33 & 2.18 & 1.82 & 87.64 & 7.47 & 2.84 & 2.06 & -2.03 & 1.14 & 0.66 & 0.24 \\
\hline Tbong Khmum & 3,382 & 21.36 & 18.31 & -3.06 & 28.92 & 25.34 & 20.25 & 25.49 & 29.39 & 35.84 & 20.55 & 14.22 & 0.47 & 10.50 & 0.30 & -11.27 \\
\hline Cambodia - All & 55,811 & 15.35 & 14.21 & -1.15 & 57.47 & 14.19 & 9.57 & 18.77 & 57.04 & 19.10 & 10.07 & 13.79 & -0.43 & 4.91 & 0.50 & -4.98 \\
\hline
\end{tabular}


Asia Pacific Region : Tree Cover on Agricultural Land (2010)

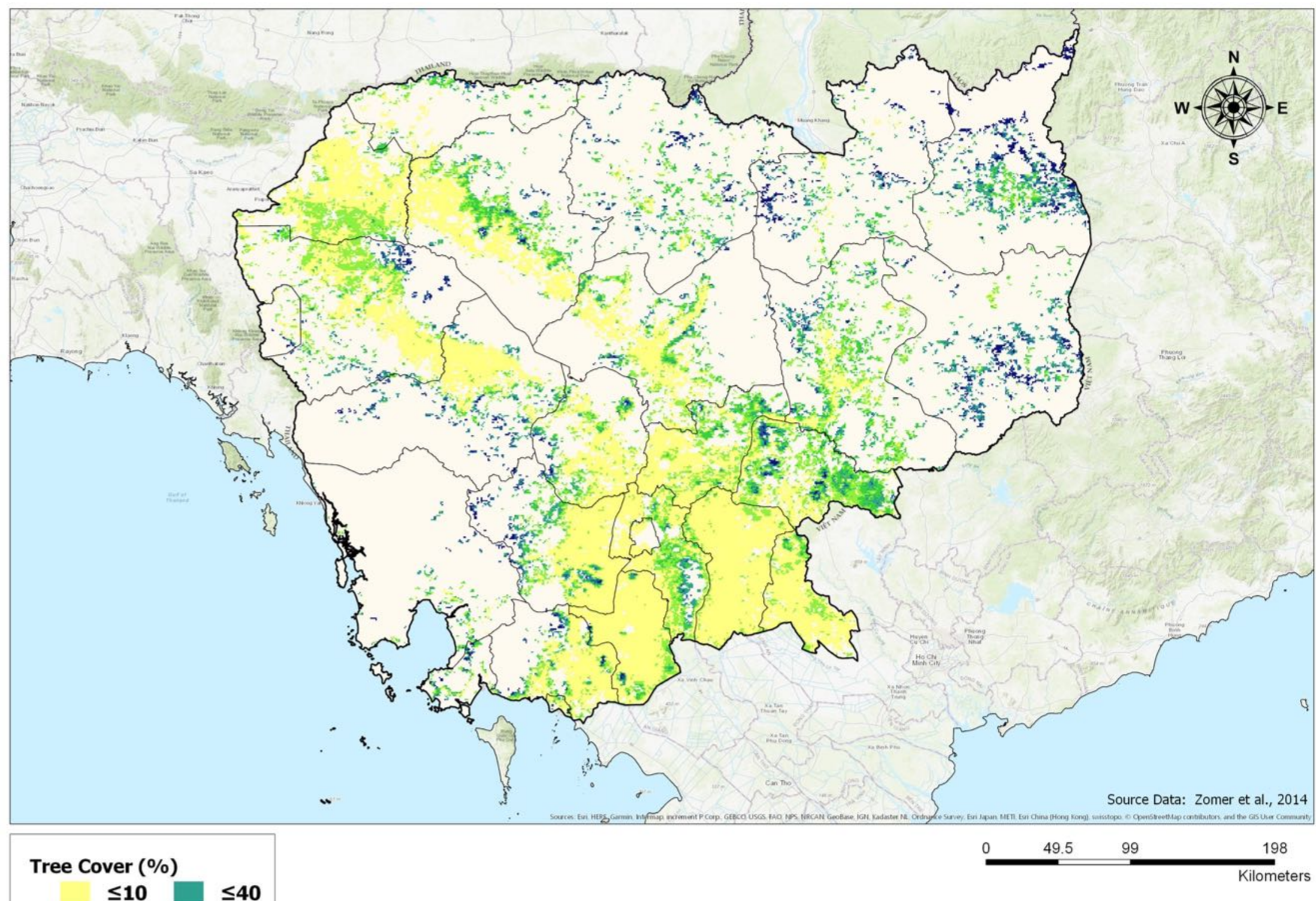

Cambodia 
Asia Pacific Region : Change in Tree Cover on Agricultural Land (2000 - 2010)

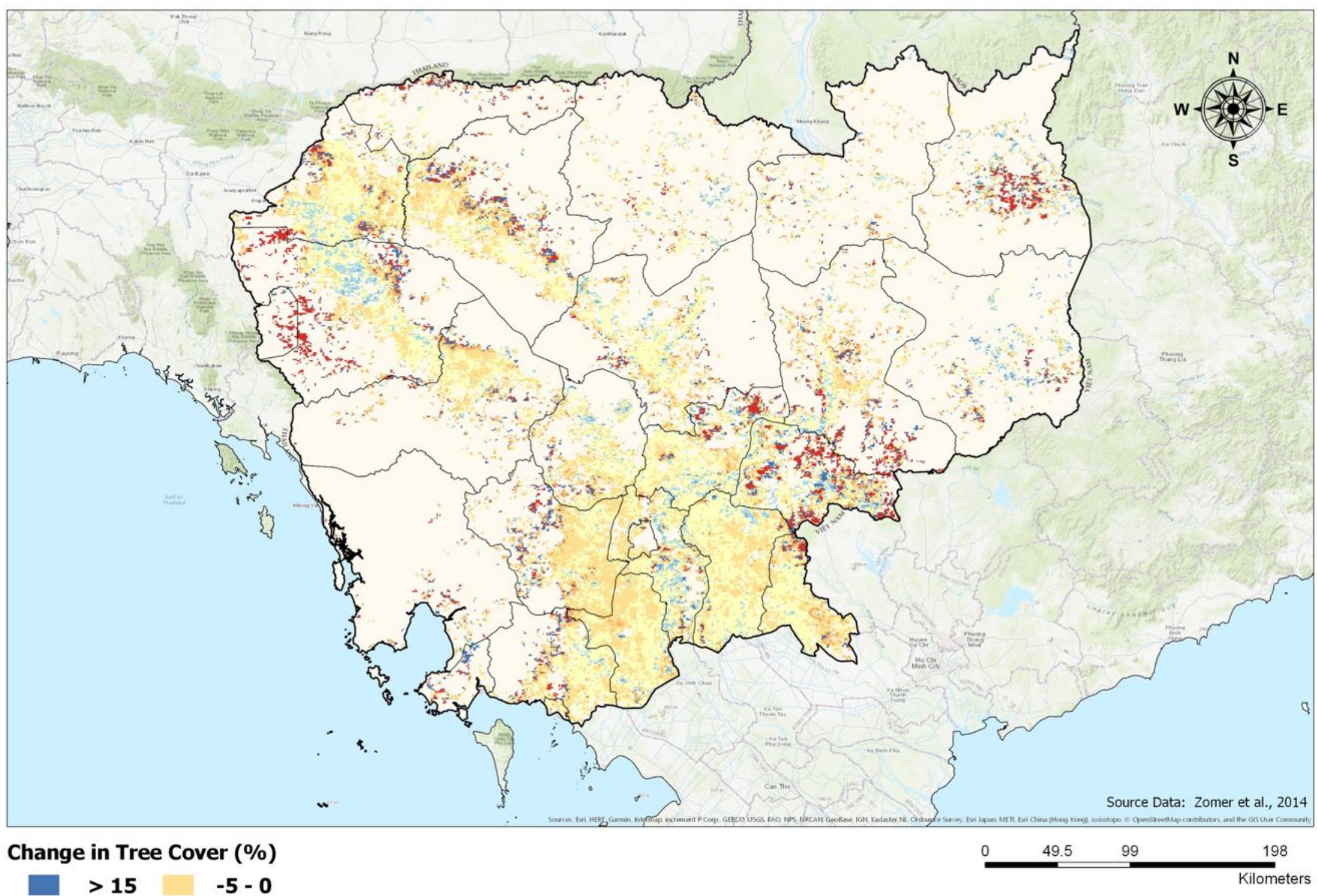

\begin{tabular}{|r|r|}
\hline$>15$ & $-5-0$ \\
$10-15$ & $-10--5$ \\
$5-10$ & $-15--10$ \\
$0-5$ & $\leq-15$
\end{tabular}

Cambodia 


\section{Appendix 6: China}

Average Percent Tree Cover by Province, and Areal Extent of Tree Cover Classes $\left(\mathrm{km}^{2}\right)$

\begin{tabular}{|c|c|c|c|c|c|c|c|c|c|c|c|c|c|c|c|c|}
\hline \multicolumn{2}{|c|}{ China } & \multirow{3}{*}{\multicolumn{3}{|c|}{\begin{tabular}{|c|} 
Average Tree Cover by Province \\
Mean Tree Cover Percent by Province \\
\end{tabular}}} & \multicolumn{12}{|c|}{ Areal Extent of Tree Cover Class $\left(\mathrm{km}^{2}\right)$} \\
\hline \multirow{3}{*}{ Province } & \multirow{3}{*}{ Area_km } & & & & \multirow{2}{*}{\multicolumn{4}{|c|}{$\begin{array}{c}\text { Year } 2000 \\
\text { Area by Tree Cover Class }\left(\mathrm{km}^{2}\right)\end{array}$}} & \multirow{2}{*}{\multicolumn{4}{|c|}{$\begin{array}{c}\text { Year } 2010 \\
\text { Area by Tree Cover Class }\left(\mathrm{km}^{2}\right)\end{array}$}} & & Change (2 & 00 to 2010$)$ & \\
\hline & & & & & & & & & & & & & \multicolumn{4}{|c|}{ Area by Tree Cover Class $\left(\mathrm{km}^{2}\right)$} \\
\hline & & 2000 & 2010 & Change & $<10 \%$ & $10-20 \%$ & $20-30 \%$ & $>30 \%$ & $<10 \%$ & $10-20 \%$ & $20-30 \%$ & $>30 \%$ & $<10 \%$ & $10-20 \%$ & $20-30 \%$ & $>30 \%$ \\
\hline Anhui & 73,237 & 11.50 & 13.46 & 1.95 & 42,177 & 25,580 & 3,821 & 1,659 & 25,118 & 41,505 & 4,553 & 2,061 & $-17,059$ & 15,925 & 732 & 402 \\
\hline Beijing & 3,619 & 6.76 & 8.15 & 1.38 & 3,165 & 341 & 94 & 19 & 2,896 & 455 & 205 & 63 & -269 & 114 & 111 & 44 \\
\hline Chongqing & 43,411 & 24.15 & 28.22 & 4.07 & 265 & 15,780 & 19,182 & 8,184 & 329 & 9,461 & 17,876 & 15,745 & 64 & $-6,319$ & $-1,306$ & 7,561 \\
\hline Fujian & 8,871 & 30.55 & 33.05 & 2.50 & 1,151 & 1,751 & 1,570 & 4,399 & 880 & 1,259 & 1,661 & 5,071 & -271 & -492 & 91 & 672 \\
\hline Gansu & 51,576 & 5.06 & 7.56 & 2.50 & 46,207 & 3,107 & 1,361 & 901 & 41,582 & 4,973 & 2,417 & 2,604 & $-4,625$ & 1,866 & 1,056 & 1,703 \\
\hline Guangdong & 24,480 & 19.95 & 22.03 & 2.08 & 4,494 & 10,856 & 5,027 & 4,103 & 3,323 & 9,623 & 6,349 & 5,185 & $-1,171$ & $-1,233$ & 1,322 & 1,082 \\
\hline Guangxi & 38,977 & 21.51 & 23.81 & 2.31 & 6,762 & 14,947 & 8,350 & 8,918 & 7,477 & 11,131 & 8,336 & 12,033 & 715 & $-3,816$ & -14 & 3,115 \\
\hline Guizhou & 32,645 & 20.58 & 25.72 & 5.14 & 4,178 & 14,070 & 9,412 & 4,985 & 2,320 & 8,794 & 11,347 & 10,184 & $-1,858$ & $-5,276$ & 1,935 & 5,199 \\
\hline Hainan & 4,450 & 11.90 & 16.35 & 4.45 & 2,218 & 1,736 & 418 & 78 & 1,187 & 1,917 & 1,143 & 203 & $-1,031$ & 181 & 725 & 125 \\
\hline Hebei & 82,546 & 5.57 & 6.43 & 0.86 & 74,804 & 6,949 & 725 & 69 & 71,093 & 8,749 & 2,191 & 514 & $-3,711$ & 1,800 & 1,466 & 445 \\
\hline Heilongjiang & 141,144 & 8.51 & 8.59 & 0.08 & 102,946 & 31,107 & 5,264 & 1,828 & 105,989 & 29,716 & 3,399 & 2,041 & 3,043 & $-1,391$ & $-1,865$ & 213 \\
\hline Henan & 110,537 & 8.82 & 11.01 & 2.19 & 82,737 & 25,346 & 1,701 & 754 & 55,119 & 50,918 & 3,237 & 1,263 & $-27,617$ & 25,572 & 1,536 & 509 \\
\hline Hubei & 68,976 & 15.86 & 17.11 & 1.25 & 16,425 & 40,257 & 7,348 & 4,946 & 12,760 & 43,213 & 5,291 & 7,712 & $-3,665$ & 2,956 & $-2,057$ & 2,766 \\
\hline Hunan & 32,965 & 20.76 & 20.60 & -0.16 & 991 & 19,600 & 8,824 & 3,550 & 1,882 & 19,028 & 7,549 & 4,506 & 891 & -572 & $-1,275$ & 956 \\
\hline Jiangsu & 66,323 & 15.21 & 14.54 & -0.67 & 14,529 & 40,422 & 10,135 & 1,237 & 16,570 & 40,985 & 7,801 & 967 & 2,041 & 563 & $-2,334$ & -270 \\
\hline Jiangxi & 29,609 & 19.55 & 19.74 & 0.19 & 5,482 & 15,002 & 3,900 & 5,225 & 5,282 & 14,696 & 3,894 & 5,737 & -200 & -306 & -6 & 512 \\
\hline Jilin & 75,345 & 6.35 & 6.76 & 0.41 & 62,342 & 8,045 & 2,592 & 2,366 & 62,025 & 8,578 & 2,715 & 2,027 & -317 & 533 & 123 & -339 \\
\hline Liaoning & 57,416 & 6.15 & 7.61 & 1.46 & 46,710 & 7,789 & 2,116 & 801 & 43,434 & 8,806 & 3,692 & 1,484 & $-3,276$ & 1,017 & 1,576 & 683 \\
\hline Nei Mongol & 203,507 & 3.61 & 3.66 & 0.05 & 193,027 & 6,664 & 1,992 & 1,825 & 190,162 & 7,497 & 2,731 & 3,118 & $-2,865$ & 833 & 739 & 1,293 \\
\hline Ningxia Hui & 9,227 & 2.38 & 3.11 & 0.74 & 9,108 & 65 & 43 & 11 & 9,064 & 67 & 43 & 53 & -44 & 2 & 0 & 42 \\
\hline Qinghai & 1,487 & 4.89 & 5.98 & 1.09 & 1,370 & 105 & 12 & 0 & 1,280 & 177 & 19 & 11 & -90 & 72 & 7 & 11 \\
\hline Shaanxi & 52,787 & 7.26 & 10.98 & 3.73 & 42,827 & 6,404 & 2,402 & 1,154 & 34,561 & 11,372 & 3,626 & 3,228 & $-8,266$ & 4,968 & 1,224 & 2,074 \\
\hline Shandong & 105,073 & 5.40 & 6.71 & 1.32 & 98,262 & 6,001 & 695 & 116 & 89,804 & 14,036 & 1,031 & 203 & $-8,458$ & 8,035 & 336 & 87 \\
\hline Shanghai & 2,623 & 19.50 & 12.92 & -6.58 & 81 & 1,517 & 948 & 77 & 844 & 1,599 & 171 & 9 & 763 & 82 & -777 & -68 \\
\hline Shanxi & 69,487 & 7.38 & 9.46 & 2.08 & 56,620 & 9,881 & 2,143 & 843 & 47,177 & 17,017 & 3,577 & 1,716 & $-9,443$ & 7,136 & 1,434 & 873 \\
\hline Sichuan & 109,836 & 23.29 & 28.16 & 4.86 & 2,537 & 49,315 & 39,391 & 18,593 & 2,066 & 28,456 & 40,444 & 38,870 & -471 & $-20,859$ & 1,053 & 20,277 \\
\hline Tianjin & 7,506 & 7.46 & 8.66 & 1.20 & 6,478 & 961 & 67 & 0 & 5,455 & 1,819 & 215 & 17 & $-1,023$ & 858 & 148 & 17 \\
\hline Xinjiang Uygur & 73,332 & 5.90 & 5.31 & -0.59 & 66,072 & 4,323 & 1,682 & 1,256 & 66,975 & 3,449 & 1,472 & 1,437 & 903 & -874 & -210 & 181 \\
\hline Xizang & 6,136 & 41.55 & 42.20 & 0.65 & 709 & 743 & 639 & 4,045 & 781 & 697 & 602 & 4,056 & 72 & -46 & -37 & 11 \\
\hline Yunnan & 82,086 & 21.29 & 24.93 & 3.64 & 14,890 & 31,912 & 19,012 & 16,272 & 12,398 & 24,136 & 21,442 & 24,110 & $-2,492$ & $-7,776$ & 2,430 & 7,838 \\
\hline Zhejiang & 22,541 & 24.80 & 21.69 & -3.11 & 621 & 9,012 & 7,772 & 5,136 & 2,813 & 11,574 & 3,476 & 4,678 & 2,192 & 2,562 & $-4,296$ & -458 \\
\hline China - All & $1,691,755$ & 11.36 & 12.84 & 1.48 & $1,010,187$ & 409,591 & 168,639 & 103,351 & 922,648 & 435,706 & 172,506 & 160,907 & $-87,539$ & 26,115 & 3,867 & 57,556 \\
\hline
\end{tabular}


Average Percent Tree Cover by Province, and Areal Extent of Tree Cover Classes as a Percent of the Total Agricultural Area (\%)

\begin{tabular}{|c|c|c|c|c|c|c|c|c|c|c|c|c|c|c|c|c|}
\hline \multicolumn{2}{|c|}{ China } & \multirow{3}{*}{\multicolumn{3}{|c|}{$\begin{array}{c}\text { Average Tree Cover by Province } \\
\text { Percent Tree Cover } \\
(\%)\end{array}$}} & \multicolumn{12}{|c|}{ Areal Extent of Tree Cover Class as a Percent of Total Agricultural Area (\%) } \\
\hline \multirow{3}{*}{ Province } & \multirow{3}{*}{ Area_km } & & & & \multirow{2}{*}{\multicolumn{4}{|c|}{$\begin{array}{c}\text { Year } 2000 \\
\text { Percent of Total Agrciultural Area (\%) }\end{array}$}} & \multirow{2}{*}{\multicolumn{4}{|c|}{$\begin{array}{c}\text { Year } 2010 \\
\text { Percent of Total Agrciultural Area (\%) }\end{array}$}} & \multirow{2}{*}{\multicolumn{4}{|c|}{ Change (2000 to 2010) }} \\
\hline & & & & & & & & & & & & & Perc & & & \\
\hline & & 2000 & 2010 & Change & $<10 \%$ & $10-20 \%$ & $20-30 \%$ & $>30 \%$ & $<10 \%$ & $10-20 \%$ & $20-30 \%$ & $>30 \%$ & $<10 \%$ & $10-20 \%$ & $20-30 \%$ & $>30 \%$ \\
\hline Anhui & 73,237 & 11.50 & 13.46 & 1.95 & 57.59 & 34.93 & 5.22 & 2.27 & 34.30 & 56.67 & 6.22 & 2.81 & -23.29 & 21.74 & 1.00 & 0.55 \\
\hline Beijing & 3,619 & 6.76 & 8.15 & 1.38 & 87.46 & 9.42 & 2.60 & 0.53 & 80.02 & 12.57 & 5.66 & 1.74 & -7.43 & 3.15 & 3.07 & 1.22 \\
\hline Chongqing & 43,411 & 24.15 & 28.22 & 4.07 & 0.61 & 36.35 & 44.19 & 18.85 & 0.76 & 21.79 & 41.18 & 36.27 & 0.15 & -14.56 & -3.01 & 17.42 \\
\hline Fujian & 8,871 & 30.55 & 33.05 & 2.50 & 12.97 & 19.74 & 17.70 & 49.59 & 9.92 & 14.19 & 18.72 & 57.16 & -3.05 & -5.55 & 1.03 & 7.58 \\
\hline Gansu & 51,576 & 5.06 & 7.56 & 2.50 & 89.59 & 6.02 & 2.64 & 1.75 & 80.62 & 9.64 & 4.69 & 5.05 & -8.97 & 3.62 & 2.05 & 3.30 \\
\hline Guangdong & 24,480 & 19.95 & 22.03 & 2.08 & 18.36 & 44.35 & 20.54 & 16.76 & 13.57 & 39.31 & 25.94 & 21.18 & -4.78 & -5.04 & 5.40 & 4.42 \\
\hline Guangxi & 38,977 & 21.51 & 23.81 & 2.31 & 17.35 & 38.35 & 21.42 & 22.88 & 19.18 & 28.56 & 21.39 & 30.87 & 1.83 & -9.79 & -0.04 & 7.99 \\
\hline Guizhou & 32,645 & 20.58 & 25.72 & 5.14 & 12.80 & 43.10 & 28.83 & 15.27 & 7.11 & 26.94 & 34.76 & 31.20 & -5.69 & -16.16 & 5.93 & 15.93 \\
\hline Hainan & 4,450 & 11.90 & 16.35 & 4.45 & 49.84 & 39.01 & 9.39 & 1.75 & 26.67 & 43.08 & 25.69 & 4.56 & -23.17 & 4.07 & 16.29 & 2.81 \\
\hline Hebei & 82,546 & 5.57 & 6.43 & 0.86 & 90.62 & 8.42 & 0.88 & 0.08 & 86.12 & 10.60 & 2.65 & 0.62 & -4.50 & 2.18 & 1.78 & 0.54 \\
\hline Heilongjiang & 141,144 & 8.51 & 8.59 & 0.08 & 72.94 & 22.04 & 3.73 & 1.30 & 75.09 & 21.05 & 2.41 & 1.45 & 2.16 & -0.99 & -1.32 & 0.15 \\
\hline Henan & 110,537 & 8.82 & 11.01 & 2.19 & 74.85 & 22.93 & 1.54 & 0.68 & 49.87 & 46.06 & 2.93 & 1.14 & -24.98 & 23.13 & 1.39 & 0.46 \\
\hline Hubei & 68,976 & 15.86 & 17.11 & 1.25 & 23.81 & 58.36 & 10.65 & 7.17 & 18.50 & 62.65 & 7.67 & 11.18 & -5.31 & 4.29 & -2.98 & 4.01 \\
\hline Hunan & 32,965 & 20.76 & 20.60 & -0.16 & 3.01 & 59.46 & 26.77 & 10.77 & 5.71 & 57.72 & 22.90 & 13.67 & 2.70 & -1.74 & -3.87 & 2.90 \\
\hline Jiangsu & 66,323 & 15.21 & 14.54 & -0.67 & 21.91 & 60.95 & 15.28 & 1.87 & 24.98 & 61.80 & 11.76 & 1.46 & 3.08 & 0.85 & -3.52 & -0.41 \\
\hline Jiangxi & 29,609 & 19.55 & 19.74 & 0.19 & 18.51 & 50.67 & 13.17 & 17.65 & 17.84 & 49.63 & 13.15 & 19.38 & -0.68 & -1.03 & -0.02 & 1.73 \\
\hline Jilin & 75,345 & 6.35 & 6.76 & 0.41 & 82.74 & 10.68 & 3.44 & 3.14 & 82.32 & 11.39 & 3.60 & 2.69 & -0.42 & 0.71 & 0.16 & -0.45 \\
\hline Liaoning & 57,416 & 6.15 & 7.61 & 1.46 & 81.35 & 13.57 & 3.69 & 1.40 & 75.65 & 15.34 & 6.43 & 2.58 & -5.71 & 1.77 & 2.74 & 1.19 \\
\hline Nei Mongol & 203,507 & 3.61 & 3.66 & 0.05 & 94.85 & 3.27 & 0.98 & 0.90 & 93.44 & 3.68 & 1.34 & 1.53 & -1.41 & 0.41 & 0.36 & 0.64 \\
\hline Ningxia Hui & 9,227 & 2.38 & 3.11 & 0.74 & 98.71 & 0.70 & 0.47 & 0.12 & 98.23 & 0.73 & 0.47 & 0.57 & -0.48 & 0.02 & 0.00 & 0.46 \\
\hline Qinghai & 1,487 & 4.89 & 5.98 & 1.09 & 92.13 & 7.06 & 0.81 & 0.00 & 86.08 & 11.90 & 1.28 & 0.74 & -6.05 & 4.84 & 0.47 & 0.74 \\
\hline Shaanxi & 52,787 & 7.26 & 10.98 & 3.73 & 81.13 & 12.13 & 4.55 & 2.19 & 65.47 & 21.54 & 6.87 & 6.12 & -15.66 & 9.41 & 2.32 & 3.93 \\
\hline Shandong & 105,073 & 5.40 & 6.71 & 1.32 & 93.52 & 5.71 & 0.66 & 0.11 & 85.47 & 13.36 & 0.98 & 0.19 & -8.05 & 7.65 & 0.32 & 0.08 \\
\hline Shanghai & 2,623 & 19.50 & 12.92 & -6.58 & 3.09 & 57.83 & 36.14 & 2.94 & 32.18 & 60.96 & 6.52 & 0.34 & 29.09 & 3.13 & -29.62 & -2.59 \\
\hline Shanxi & 69,487 & 7.38 & 9.46 & 2.08 & 81.48 & 14.22 & 3.08 & 1.21 & 67.89 & 24.49 & 5.15 & 2.47 & -13.59 & 10.27 & 2.06 & 1.26 \\
\hline Sichuan & 109,836 & 23.29 & 28.16 & 4.86 & 2.31 & 44.90 & 35.86 & 16.93 & 1.88 & 25.91 & 36.82 & 35.39 & -0.43 & -18.99 & 0.96 & 18.46 \\
\hline Tianjin & 7,506 & 7.46 & 8.66 & 1.20 & 86.30 & 12.80 & 0.89 & 0.00 & 72.68 & 24.23 & 2.86 & 0.23 & -13.63 & 11.43 & 1.97 & 0.23 \\
\hline Xinjiang Uygur & 73,332 & 5.90 & 5.31 & -0.59 & 90.10 & 5.90 & 2.29 & 1.71 & 91.33 & 4.70 & 2.01 & 1.96 & 1.23 & -1.19 & -0.29 & 0.25 \\
\hline Xizang & 6,136 & 41.55 & 42.20 & 0.65 & 11.55 & 12.11 & 10.41 & 65.92 & 12.73 & 11.36 & 9.81 & 66.10 & 1.17 & -0.75 & -0.60 & 0.18 \\
\hline Yunnan & 82,086 & 21.29 & 24.93 & 3.64 & 18.14 & 38.88 & 23.16 & 19.82 & 15.10 & 29.40 & 26.12 & 29.37 & -3.04 & -9.47 & 2.96 & 9.55 \\
\hline Zhejiang & 22,541 & 24.80 & 21.69 & -3.11 & 2.76 & 39.98 & 34.48 & 22.79 & 12.48 & 51.35 & 15.42 & 20.75 & 9.72 & 11.37 & -19.06 & -2.03 \\
\hline China - All & $1,691,755$ & 11.36 & 12.84 & 1.48 & 59.71 & 24.21 & 9.97 & 6.11 & 54.54 & 25.75 & 10.20 & 9.51 & -5.17 & 1.54 & 0.23 & 3.40 \\
\hline
\end{tabular}


Asia Pacific Region : Tree Cover on Agricultural Land (2010)

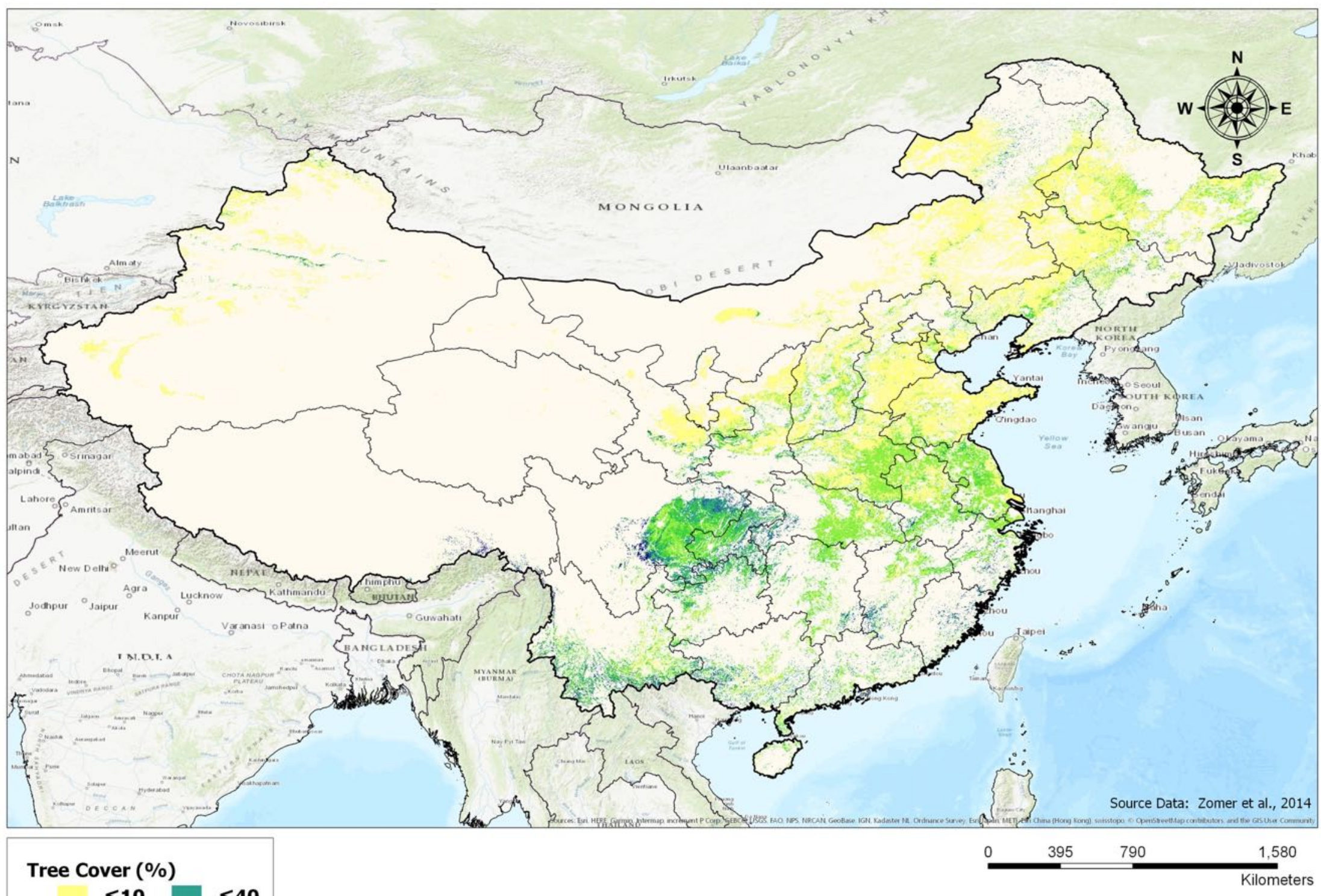
$\leq \mathbf{1 0}$
$\mathbf{2 0}$

China 
Asia Pacific Region : Change in Tree Cover on Agricultural Land (2000 - 2010)

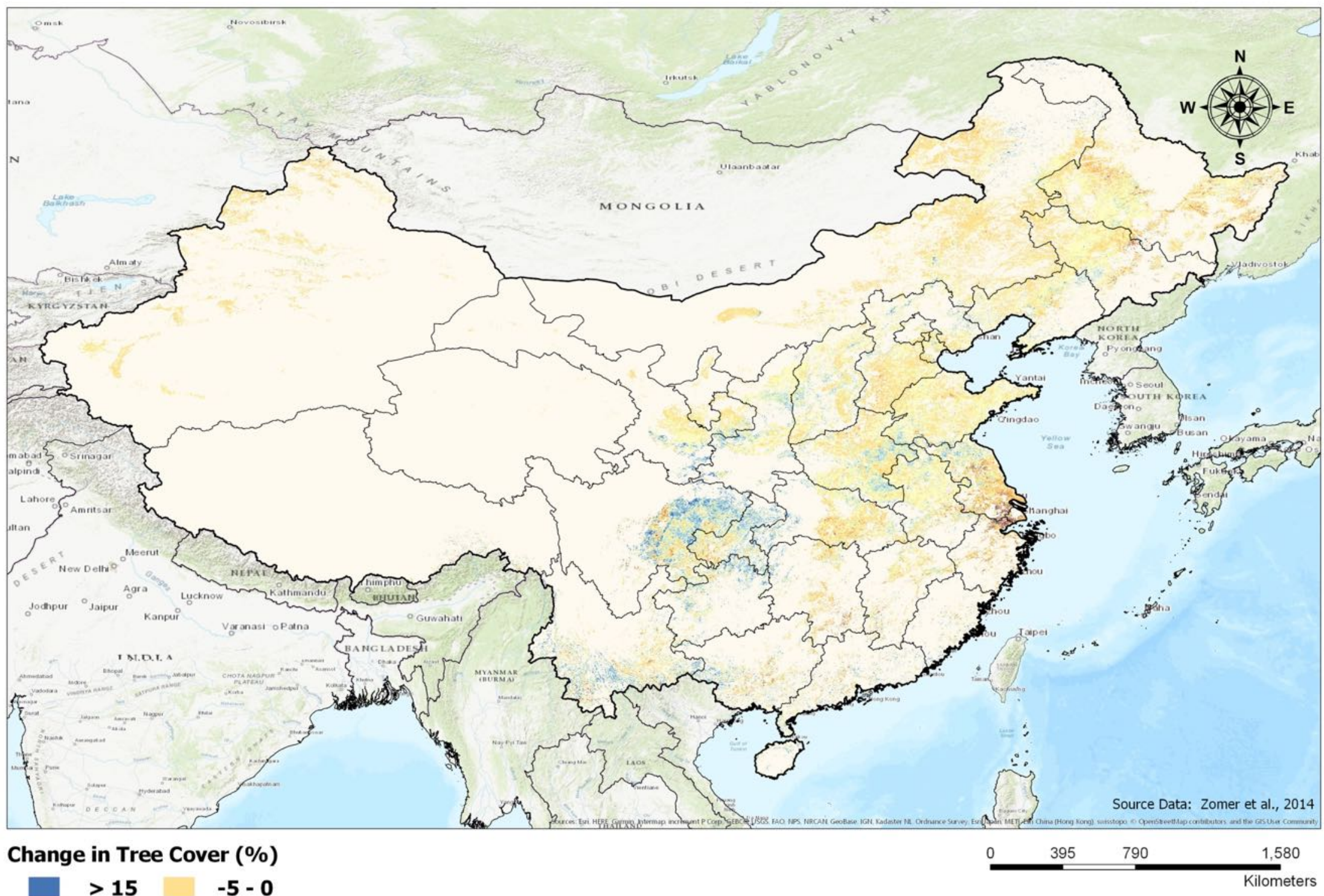

$10-15$

$\begin{aligned} 10-15 & -10--5 \\ 5-10 & -15--10\end{aligned}$

$0-5 \square \leq-15$

China 
Average Percent Tree Cover by Province, and Areal Extent of Tree Cover Classes $\left(\mathrm{km}^{2}\right)$

\begin{tabular}{|c|c|c|c|c|c|c|c|c|c|c|c|c|c|c|c|c|}
\hline \multicolumn{2}{|c|}{$\mathrm{Fiji}_{\mathrm{i}}$} & \multicolumn{3}{|c|}{ Average Tree Cover by Province } & \multicolumn{12}{|c|}{ Areal Extent of Tree Cover Class $\left(\mathrm{km}^{2}\right)$} \\
\hline \multirow{3}{*}{ Province } & \multirow{3}{*}{ Area_km } & \multirow{2}{*}{\multicolumn{3}{|c|}{$\begin{array}{c}\text { Mean Tree Cover Percent by Province } \\
(\%)\end{array}$}} & \multicolumn{4}{|c|}{$\begin{array}{l}\text { Year 2000 } \\
\end{array}$} & \multirow{2}{*}{\multicolumn{4}{|c|}{$\begin{array}{c}\text { Year } 2010 \\
\text { Area by Tree Cover Class }\left(\mathrm{km}^{2}\right)\end{array}$}} & \multirow{2}{*}{\multicolumn{4}{|c|}{$\begin{array}{c}\text { Change (2000 to 2010) } \\
\text { Area by Tree Cover Class }\left(\mathrm{km}^{2}\right)\end{array}$}} \\
\hline & & & & & & by Tree & ver Class $(k$ & & & & & & & & & \\
\hline & & 2000 & 2010 & Change & $<10 \%$ & $10-20 \%$ & $20-30 \%$ & $>30 \%$ & $<10 \%$ & $10-20 \%$ & $20-30 \%$ & $>30 \%$ & $<10 \%$ & $10-20 \%$ & $20-30 \%$ & $>30 \%$ \\
\hline Central & 219 & 55.51 & 58.10 & 2.59 & 0 & 7 & 15 & 197 & 0 & 1 & 16 & 202 & 0 & -6 & 1 & 5 \\
\hline Eastern & 56 & 68.21 & 68.86 & 0.64 & 0 & 0 & 1 & 55 & 0 & 0 & 1 & 55 & 0 & 0 & 0 & 0 \\
\hline Northern & 594 & 39.47 & 43.75 & 4.28 & 5 & 177 & 97 & 315 & 1 & 91 & 154 & 348 & -4 & -86 & 57 & 33 \\
\hline Western & 1,917 & 29.83 & 36.53 & 6.71 & 89 & 627 & 482 & 719 & 0 & 293 & 591 & 1,033 & -89 & -334 & 109 & 314 \\
\hline Fiji - All & 2,786 & 34.67 & 40.42 & 5.74 & 94 & 811 & 595 & 1,286 & 1 & 385 & 762 & 1,638 & $\begin{array}{l}-93 \\
\end{array}$ & -426 & 167 & 352 \\
\hline
\end{tabular}

Average Percent Tree Cover by Province, and Areal Extent of Tree Cover Classes as a Percent of the Total Agricultural Area (\%)

\begin{tabular}{|c|c|c|c|c|c|c|c|c|c|c|c|c|c|c|c|c|}
\hline \multicolumn{2}{|c|}{ Fiji } & \multirow{3}{*}{\multicolumn{3}{|c|}{$\begin{array}{c}\text { Average Tree Cover by Province } \\
\text { Percent Tree Cover } \\
(\%)\end{array}$}} & \multicolumn{12}{|c|}{ Areal Extent of Tree Cover Class as a Percent of Total Agricultural Area (\%) } \\
\hline \multirow{3}{*}{ Province } & \multirow{3}{*}{ Area_km } & & & & \multirow{2}{*}{\multicolumn{4}{|c|}{$\begin{array}{c}\text { Year } 2000 \\
\text { Percent of Total Agrciultural Area (\%) }\end{array}$}} & & Yea & 0010 & & & Change (2 & 0 to 2010) & \\
\hline & & & & & & & & & \multicolumn{4}{|c|}{ Percent of Total Agrciultural Area (\%) } & \multicolumn{4}{|c|}{ Percent of Total Agrciultural Area ( $\%)$} \\
\hline & & 2000 & 2010 & Change & $<10 \%$ & $10-20 \%$ & $20-30 \%$ & $>30 \%$ & $<10 \%$ & $10-20 \%$ & $20-30 \%$ & $>30 \%$ & $<10 \%$ & $10-20 \%$ & $20-30 \%$ & $>30 \%$ \\
\hline Central & 219 & 55.51 & 58.10 & 2.59 & 0.00 & 3.20 & 6.85 & 89.96 & 0.00 & 0.46 & 7.31 & 92.24 & 0.00 & -2.74 & 0.46 & 2.28 \\
\hline Eastern & 56 & 68.21 & 68.86 & 0.64 & 0.00 & 0.00 & 1.79 & 98.22 & 0.00 & 0.00 & 1.79 & 98.22 & 0.00 & 0.00 & 0.00 & 0.00 \\
\hline Northern & 594 & 39.47 & 43.75 & 4.28 & 0.84 & 29.80 & 16.33 & 53.03 & 0.17 & 15.32 & 25.93 & 58.59 & -0.67 & -14.48 & 9.60 & 5.56 \\
\hline Western & 1,917 & 29.83 & 36.53 & 6.71 & 4.64 & 32.71 & 25.14 & 37.51 & 0.00 & 15.28 & 30.83 & 53.89 & -4.64 & -17.42 & 5.69 & 16.38 \\
\hline Fij - All & 2,786 & 34.67 & 40.42 & 5.74 & 3.37 & 29.11 & 21.36 & 46.16 & 0.04 & 13.82 & 27.35 & 58.79 & -3.34 & -15.29 & 5.99 & 12.63 \\
\hline
\end{tabular}


Asia Pacific Region : Tree Cover on Agricultural Land (2010)

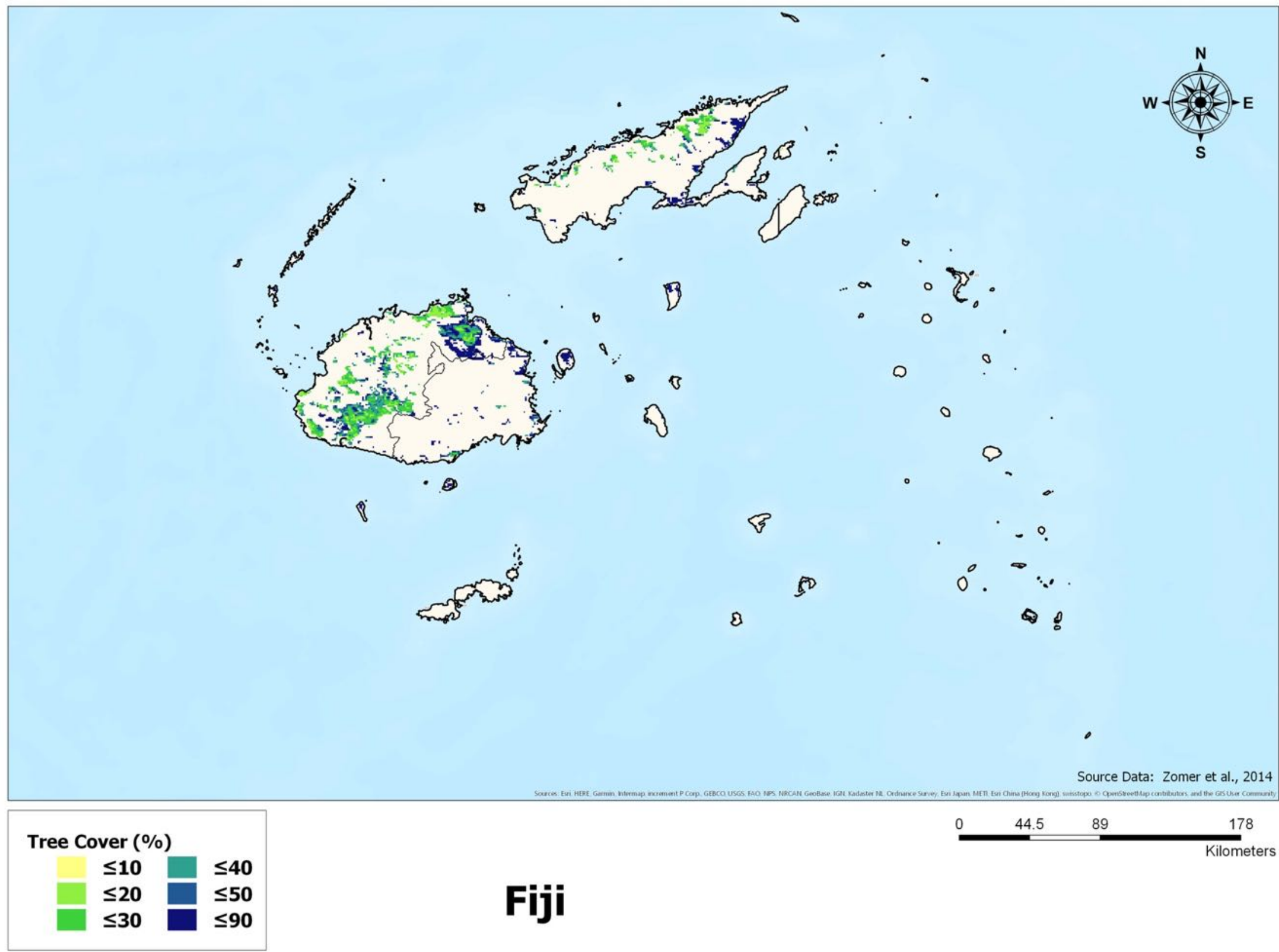


Asia Pacific Region : Change in Tree Cover on Agricultural Land (2000 - 2010)

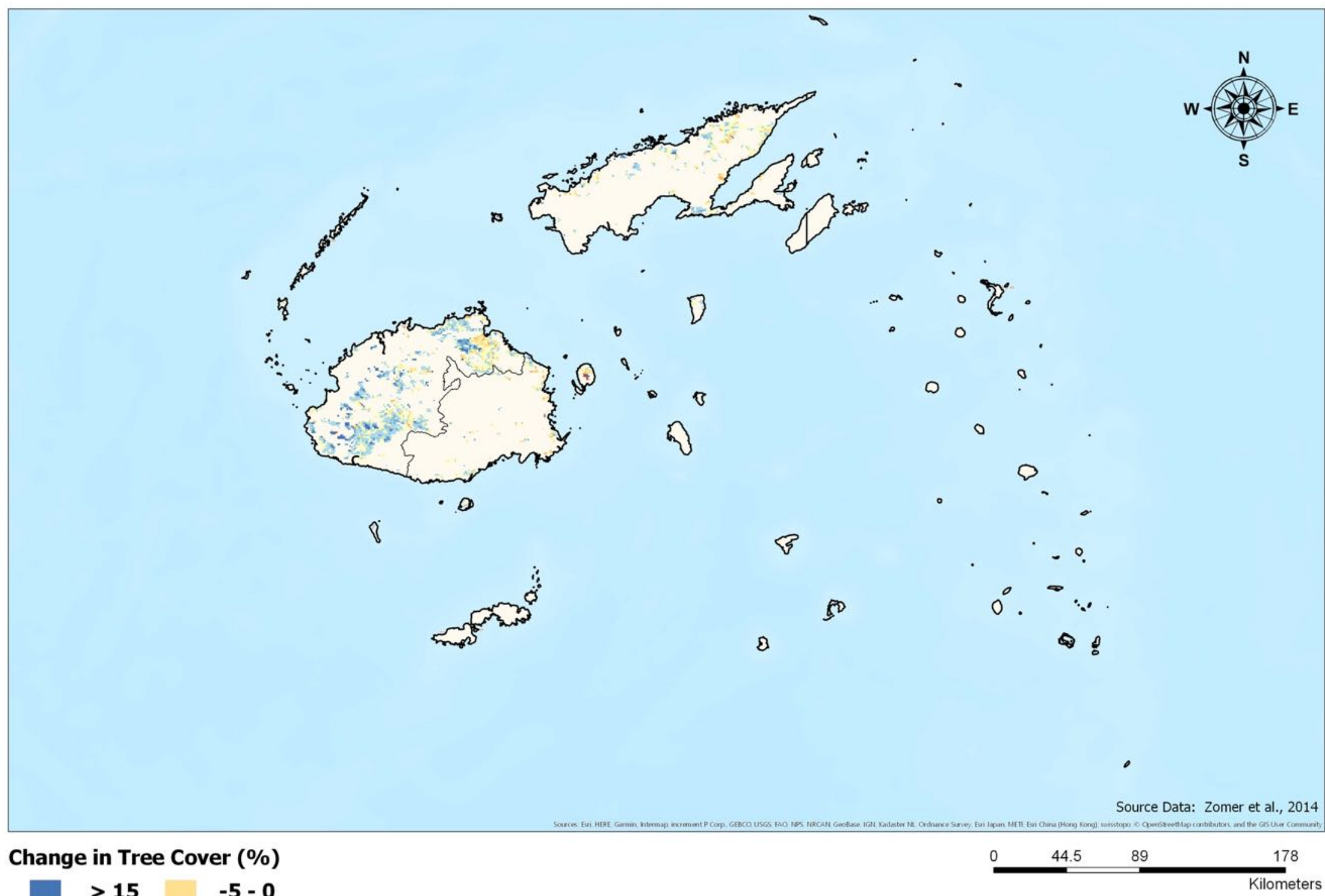

Fiji 
Appendix 8: India

Average Percent Tree Cover by Province, and Areal Extent of Tree Cover Classes $\left(\mathrm{km}^{2}\right)$

\begin{tabular}{|c|c|c|c|c|c|c|c|c|c|c|c|c|c|c|c|c|}
\hline \multicolumn{2}{|l|}{ India } & \multirow{3}{*}{\multicolumn{3}{|c|}{\begin{tabular}{|c|} 
Average Tree Cover by Province \\
Mean Tree Cover Percent by Province
\end{tabular}}} & \multicolumn{12}{|c|}{ Areal Extent of Tree Cover Class $\left(\mathrm{km}^{2}\right)$} \\
\hline \multirow{3}{*}{ State } & \multirow{3}{*}{ Area_km } & & & & \multirow{2}{*}{\multicolumn{4}{|c|}{$\begin{array}{c}\text { Year } 2000 \\
\text { Area by Tree Cover Class }\left(\mathrm{km}^{2}\right)\end{array}$}} & \multirow{2}{*}{\multicolumn{4}{|c|}{$\begin{array}{c}\text { Year } 2010 \\
\text { Area by Tree Cover Class }\left(\mathrm{km}^{2}\right)\end{array}$}} & \multirow{2}{*}{\multicolumn{4}{|c|}{$\begin{array}{c}\text { Change }(2000 \text { to } 2010) \\
\text { Area by Tree Cover Class }\left(\mathrm{km}^{2}\right)\end{array}$}} \\
\hline & & & & & & & & & & & & & & & & \\
\hline & & 2000 & 2010 & Change & $<10 \%$ & $10-20 \%$ & $20-30 \%$ & $>30 \%$ & $<10 \%$ & $10-20 \%$ & $20-30 \%$ & $>30 \%$ & $<10 \%$ & $10-20 \%$ & $20-30 \%$ & $>30 \%$ \\
\hline Andaman and Nicobar & 160 & 35.32 & 38.11 & 2.79 & 0 & 28 & 45 & 87 & 3 & 18 & 40 & 99 & 3 & -10 & -5 & 12 \\
\hline Andhra Pradesh & 92,528 & 6.58 & 7.80 & 1.22 & 72,823 & 15,825 & 3,490 & 391 & 65,702 & 23,198 & 3,270 & 358 & $-7,120$ & 7,373 & -220 & -33 \\
\hline Arunachal Pradesh & 15,482 & 50.53 & 51.55 & 1.02 & 1,178 & 1,347 & 1,209 & 11,748 & 1,222 & 1,080 & 1,333 & 11,847 & 44 & -267 & 124 & 99 \\
\hline Assam & 50,188 & 14.66 & 17.61 & 2.95 & 22,620 & 19,271 & 3,512 & 4,785 & 11,200 & 26,869 & 7,494 & 4,625 & $-11,420$ & 7,598 & 3,982 & -160 \\
\hline Bihar & 79,137 & 8.62 & 7.03 & -1.59 & 53,443 & 25,085 & 510 & 99 & 68,681 & 10,204 & 230 & 23 & 15,237 & $-14,881$ & -280 & -76 \\
\hline Chhattisgarh & 62,228 & 4.98 & 4.84 & -0.13 & 56,255 & 4,785 & 1,084 & 104 & 57,785 & 4,278 & 165 & 0 & 1,530 & -507 & -919 & -104 \\
\hline Dadra and Nagar Haveli & 217 & 4.49 & 6.20 & 1.71 & 201 & 16 & 0 & 0 & 200 & 17 & 0 & 0 & -1 & 1 & 0 & 0 \\
\hline Goa & 561 & 15.63 & 19.07 & 3.44 & 178 & 233 & 117 & 33 & 76 & 236 & 218 & 31 & -102 & 3 & 101 & -2 \\
\hline Gujarat & 85,398 & 2.74 & 4.53 & 1.79 & 84,637 & 724 & 36 & 2 & 82,131 & 3,205 & 63 & 0 & $-2,506$ & 2,481 & 27 & -2 \\
\hline Haryana & 37,403 & 5.78 & 5.98 & 0.20 & 30,275 & 6,719 & 388 & 21 & 30,399 & 6,901 & 101 & 2 & 124 & 182 & -287 & -19 \\
\hline Himachal Pradesh & 14,057 & 19.07 & 19.83 & 0.76 & 2,820 & 5,201 & 4,696 & 1,340 & 2,346 & 4,867 & 5,662 & 1,182 & -474 & -334 & 966 & -158 \\
\hline Jammu and Kashmir & 17,579 & 16.48 & 19.15 & 2.66 & 6,330 & 6,199 & 3,192 & 1,858 & 4,354 & 6,624 & 4,022 & 2,579 & $-1,976$ & 425 & 830 & 721 \\
\hline Jharkhand & 42,430 & 4.30 & 4.56 & 0.26 & 38,800 & 3,046 & 564 & 20 & 39,515 & 2,776 & 139 & 0 & 715 & -270 & -425 & -20 \\
\hline Karnataka & 84,925 & 4.55 & 6.05 & 1.51 & 78,848 & 3,538 & 1,714 & 826 & 75,151 & 6,746 & 2,192 & 837 & $-3,697$ & 3,208 & 478 & 11 \\
\hline Kerala & 8,074 & 32.05 & 29.84 & -2.21 & 93 & 778 & 2,520 & 4,683 & 52 & 1,046 & 3,606 & 3,370 & -41 & 268 & 1,086 & $-1,313$ \\
\hline Madhya Pradesh & 154,040 & 5.14 & 7.07 & 1.93 & 146,969 & 6,405 & 648 & 19 & 122,298 & 31,699 & 44 & 0 & $-24,671$ & 25,294 & -604 & -19 \\
\hline Maharashtra & 169,509 & 6.39 & 8.79 & 2.39 & 153,539 & 14,544 & 1,100 & 327 & 124,698 & 43,257 & 1,212 & 343 & $-28,841$ & 28,713 & 112 & 16 \\
\hline Manipur & 1,632 & 31.16 & 27.06 & -4.10 & 499 & 347 & 54 & 732 & 541 & 294 & 99 & 698 & 42 & -53 & 45 & -34 \\
\hline Meghalaya & 410 & 18.40 & 20.40 & 2.00 & 186 & 88 & 47 & 89 & 81 & 176 & 77 & 76 & -105 & 88 & 30 & -13 \\
\hline Mizoram & 3,032 & 57.47 & 51.43 & -6.04 & 0 & 0 & 16 & 3,016 & 0 & 0 & 23 & 3,009 & 0 & 0 & 7 & -7 \\
\hline Nagaland & 982 & 43.46 & 47.83 & 4.37 & 64 & 119 & 79 & 720 & 37 & 122 & 69 & 754 & -27 & 3 & -10 & 34 \\
\hline NCT of Delhi & 87 & 3.77 & 4.39 & 0.62 & 87 & 0 & 0 & 0 & 87 & 0 & 0 & 0 & 0 & 0 & 0 & $\begin{array}{r}04 \\
0\end{array}$ \\
\hline Odisha & 70,348 & 4.99 & 6.89 & 1.90 & 63,698 & 5,283 & 1,058 & 309 & 56,701 & 12,048 & 1,381 & 218 & $-6,997$ & 6,765 & 323 & -91 \\
\hline Puducherry & 107 & 14.47 & 17.14 & 2.67 & 53 & 28 & 16 & 10 & 10 & 73 & 19 & 5 & -43 & 45 & 3 & -5 \\
\hline Punjab & 42,527 & 10.80 & 10.85 & 0.05 & 21,737 & 19,181 & 1,542 & 67 & 19,017 & 22,833 & 674 & 3 & $-2,720$ & 3,652 & -868 & -64 \\
\hline Rajasthan & 202,275 & 1.75 & 2.22 & 0.47 & 202,054 & 223 & 0 & 0 & 199,682 & 2,588 & 7 & 0 & $-2,372$ & 2,365 & 7 & 0 \\
\hline Sikkim & 582 & 45.21 & 50.20 & 4.99 & 30 & 38 & 105 & 409 & 28 & 20 & 49 & 485 & -2 & -18 & -56 & 76 \\
\hline Tamil Nadu & 72,544 & 6.03 & 8.26 & 2.23 & 61,905 & 8,994 & 1,025 & 620 & 54,853 & 14,817 & 1,969 & 905 & $-7,052$ & 5,823 & 944 & 285 \\
\hline Telangana & 55,227 & 5.32 & 5.97 & 0.65 & 50,100 & 4,166 & 861 & 100 & 48,182 & 6,645 & 392 & 8 & $-1,918$ & 2,479 & -469 & -92 \\
\hline Tripura & 2,597 & 39.49 & 35.58 & -3.91 & 204 & 307 & 227 & 1,859 & 32 & 529 & 433 & 1,603 & -172 & 222 & 206 & -256 \\
\hline Uttar Pradesh & 193,655 & 6.51 & 5.41 & -1.10 & 161,417 & 29,626 & 1,925 & 688 & 175,705 & 16,543 & 1,053 & 355 & 14,288 & $-13,083$ & -872 & -333 \\
\hline Uttarakhand & 15,506 & 21.68 & 21.49 & -0.19 & 3,150 & 5,742 & 3,070 & 3,544 & 2,771 & 5,497 & 4,055 & 3,183 & -379 & -245 & 985 & -361 \\
\hline West Bengal & 64,704 & 7.39 & 10.94 & 3.55 & 52,107 & 10,422 & 1,226 & 949 & 34,724 & 26,157 & 2,756 & 1,067 & $-17,383$ & 15,735 & 1,530 & 118 \\
\hline India - All & $1,640,132$ & 6.84 & 7.82 & 0.98 & \begin{tabular}{|l|}
$1,366,304$ \\
\end{tabular} & 198,310 & 36,075 & 39,455 & $1,278,266$ & 281,367 & 42,847 & 37,664 & $-88,039$ & 83,058 & 6,772 & $-1,791$ \\
\hline
\end{tabular}


Average Percent Tree Cover by Province, and Areal Extent of Tree Cover Classes as a Percent of the Total Agricultural Area (\%)

\begin{tabular}{|c|c|c|c|c|c|c|c|c|c|c|c|c|c|c|c|c|}
\hline \multicolumn{2}{|l|}{ India } & \multirow{3}{*}{\multicolumn{3}{|c|}{$\begin{array}{c}\text { Average Tree Cover by Province } \\
\text { Percent Tree Cover } \\
(\%)\end{array}$}} & \multicolumn{12}{|c|}{ Areal Extent of Tree Cover Class as a Percent of Total Agricultural Area (\%) } \\
\hline \multirow{3}{*}{ State } & \multirow{3}{*}{ Area_km } & & & & \multirow{2}{*}{\multicolumn{4}{|c|}{$\begin{array}{c}\text { Year } 2000 \\
\text { Percent of Total Agrciultural Area (\%) }\end{array}$}} & & Year & 2010 & & & Change $(2)$ & 0 to 2010) & \\
\hline & & & & & & & & & \multicolumn{4}{|c|}{ Percent of Total Agrciultural Area (\%) } & \multicolumn{4}{|c|}{ Percent of Total Agrciultural Area (\%) } \\
\hline & & 2000 & 2010 & Change & $<10 \%$ & $10-20 \%$ & $20-30 \%$ & $>30 \%$ & $<10 \%$ & $10-20 \%$ & $20-30 \%$ & $>30 \%$ & $<10 \%$ & $10-20 \%$ & $20-30 \%$ & $>30 \%$ \\
\hline Andaman and Nicobar & 160 & 35.32 & 38.11 & 2.79 & 0.00 & 17.50 & 28.13 & 54.38 & 1.88 & 11.25 & 25.00 & 61.88 & 1.88 & -6.25 & -3.13 & 7.50 \\
\hline Andhra Pradesh & 92,528 & 6.58 & 7.80 & 1.22 & 78.70 & 17.10 & 3.77 & 0.42 & 71.01 & 25.07 & 3.53 & 0.39 & -7.70 & 7.97 & -0.24 & -0.04 \\
\hline Arunachal Pradesh & 15,482 & 50.53 & 51.55 & 1.02 & 7.61 & 8.70 & 7.81 & 75.88 & 7.89 & 6.98 & 8.61 & 76.52 & 0.28 & -1.72 & 0.80 & 0.64 \\
\hline Assam & 50,188 & 14.66 & 17.61 & 2.95 & 45.07 & 38.40 & 7.00 & 9.53 & 22.32 & 53.54 & 14.93 & 9.22 & -22.75 & 15.14 & 7.93 & -0.32 \\
\hline Bihar & 79,137 & 8.62 & 7.03 & -1.59 & 67.53 & 31.70 & 0.64 & 0.13 & 86.79 & 12.89 & 0.29 & 0.03 & 19.25 & -18.80 & -0.35 & -0.10 \\
\hline Chhattisgarh & 62,228 & 4.98 & 4.84 & -0.13 & 90.40 & 7.69 & 1.74 & 0.17 & 92.86 & 6.87 & 0.27 & 0.00 & 2.46 & -0.81 & -1.48 & -0.17 \\
\hline Dadra and Nagar Havel & 217 & 4.49 & 6.20 & 1.71 & 92.63 & 7.37 & 0.00 & 0.00 & 92.17 & 7.83 & 0.00 & 0.00 & -0.46 & 0.46 & 0.00 & 0.00 \\
\hline Goa & 561 & 15.63 & 19.07 & 3.44 & 31.73 & 41.53 & 20.86 & 5.88 & 13.55 & 42.07 & 38.86 & 5.53 & -18.18 & 0.53 & 18.00 & -0.36 \\
\hline Gujarat & 85,398 & 2.74 & 4.53 & 1.79 & 99.11 & 0.85 & 0.04 & 0.00 & 96.17 & 3.75 & 0.07 & 0.00 & -2.93 & 2.91 & 0.03 & 0.00 \\
\hline Haryana & 37,403 & 5.78 & 5.98 & 0.20 & 80.94 & 17.96 & 1.04 & 0.06 & 81.27 & 18.45 & 0.27 & 0.01 & 0.33 & 0.49 & -0.77 & -0.05 \\
\hline Himachal Pradesh & 14,057 & 19.07 & 19.83 & 0.76 & 20.06 & 37.00 & 33.41 & 9.53 & 16.69 & 34.62 & 40.28 & 8.41 & -3.37 & -2.38 & 6.87 & -1.12 \\
\hline Jammu and Kashmir & 17,579 & 16.48 & 19.15 & 2.66 & 36.01 & 35.26 & 18.16 & 10.57 & 24.77 & 37.68 & 22.88 & 14.67 & -11.24 & 2.42 & 4.72 & 4.10 \\
\hline Jharkhand & 42,430 & 4.30 & 4.56 & 0.26 & 91.45 & 7.18 & 1.33 & 0.05 & 93.13 & 6.54 & 0.33 & 0.00 & 1.69 & -0.64 & -1.00 & -0.05 \\
\hline Karnataka & 84,925 & 4.55 & 6.05 & 1.51 & 92.84 & 4.17 & 2.02 & 0.97 & 88.49 & 7.94 & 2.58 & 0.99 & -4.35 & 3.78 & 0.56 & 0.01 \\
\hline Kerala & 8,074 & 32.05 & 29.84 & -2.21 & 1.15 & 9.64 & 31.21 & 58.00 & 0.64 & 12.96 & 44.66 & 41.74 & -0.51 & 3.32 & 13.45 & -16.26 \\
\hline Madhya Pradesh & 154,040 & 5.14 & 7.07 & 1.93 & 95.41 & 4.16 & 0.42 & 0.01 & 79.39 & 20.58 & 0.03 & 0.00 & -16.02 & 16.42 & -0.39 & -0.01 \\
\hline Maharashtra & 169,509 & 6.39 & 8.79 & 2.39 & 90.58 & 8.58 & 0.65 & 0.19 & 73.56 & 25.52 & 0.72 & 0.20 & -17.01 & 16.94 & 0.07 & 0.01 \\
\hline Manipur & 1,632 & 31.16 & 27.06 & -4.10 & 30.58 & 21.26 & 3.31 & 44.85 & 33.15 & 18.01 & 6.07 & 42.77 & 2.57 & -3.25 & 2.76 & -2.08 \\
\hline Meghalaya & 410 & 18.40 & 20.40 & 2.00 & 45.37 & 21.46 & 11.46 & 21.71 & 19.76 & 42.93 & 18.78 & 18.54 & -25.61 & 21.46 & 7.32 & -3.17 \\
\hline Mizoram & 3,032 & 57.47 & 51.43 & -6.04 & 0.00 & 0.00 & 0.53 & 99.47 & 0.00 & 0.00 & 0.76 & 99.24 & 0.00 & 0.00 & 0.23 & -0.23 \\
\hline Nagaland & 982 & 43.46 & 47.83 & 4.37 & 6.52 & 12.12 & 8.04 & 73.32 & 3.77 & 12.42 & 7.03 & 76.78 & -2.75 & 0.31 & -1.02 & 3.46 \\
\hline NCT of Delhi & 87 & 3.77 & 4.39 & 0.62 & 100.00 & 0.00 & 0.00 & 0.00 & 100.00 & 0.00 & 0.00 & 0.00 & 0.00 & 0.00 & 0.00 & 0.00 \\
\hline Odisha & 70,348 & 4.99 & 6.89 & 1.90 & 90.55 & 7.51 & 1.50 & 0.44 & 80.60 & 17.13 & 1.96 & 0.31 & -9.95 & 9.62 & 0.46 & -0.13 \\
\hline Puducherry & 107 & 14.47 & 17.14 & 2.67 & 49.53 & 26.17 & 14.95 & 9.35 & 9.35 & 68.22 & 17.76 & 4.67 & -40.19 & 42.06 & 2.80 & -4.67 \\
\hline Punjab & 42,527 & 10.80 & 10.85 & 0.05 & 51.11 & 45.10 & 3.63 & 0.16 & 44.72 & 53.69 & 1.58 & 0.01 & -6.40 & 8.59 & -2.04 & -0.15 \\
\hline Rajasthan & 202,275 & 1.75 & 2.22 & 0.47 & 99.89 & 0.11 & 0.00 & 0.00 & 98.72 & 1.28 & 0.00 & 0.00 & -1.17 & 1.17 & 0.00 & 0.00 \\
\hline Sikkim & 582 & 45.21 & 50.20 & 4.99 & 5.15 & 6.53 & 18.04 & 70.28 & 4.81 & 3.44 & 8.42 & 83.33 & -0.34 & -3.09 & -9.62 & 13.06 \\
\hline Tamil Nadu & 72,544 & 6.03 & 8.26 & 2.23 & 85.34 & 12.40 & 1.41 & 0.85 & 75.61 & 20.43 & 2.71 & 1.25 & -9.72 & 8.03 & 1.30 & 0.39 \\
\hline Telangana & 55,227 & 5.32 & 5.97 & 0.65 & 90.72 & 7.54 & 1.56 & 0.18 & 87.24 & 12.03 & 0.71 & 0.01 & -3.47 & 4.49 & -0.85 & -0.17 \\
\hline Tripura & 2,597 & 39.49 & 35.58 & -3.91 & 7.86 & 11.82 & 8.74 & 71.58 & 1.23 & 20.37 & 16.67 & 61.73 & -6.62 & 8.55 & 7.93 & -9.86 \\
\hline Uttar Pradesh & 193,655 & 6.51 & 5.41 & -1.10 & 83.35 & 15.30 & 0.99 & 0.36 & 90.73 & 8.54 & 0.54 & 0.18 & 7.38 & -6.76 & -0.45 & -0.17 \\
\hline Uttarakhand & 15,506 & 21.68 & 21.49 & -0.19 & 20.31 & 37.03 & 19.80 & 22.86 & 17.87 & 35.45 & 26.15 & 20.53 & -2.44 & -1.58 & 6.35 & -2.33 \\
\hline West Bengal & 64,704 & 7.39 & 10.94 & 3.55 & 80.53 & 16.11 & 1.89 & 1.47 & 53.67 & 40.43 & 4.26 & 1.65 & -26.87 & 24.32 & 2.36 & 0.18 \\
\hline India - All & $1,640,132$ & 6.84 & 7.82 & 0.98 & 83.30 & 12.09 & 2.20 & 2.41 & 77.94 & 17.16 & 2.61 & 2.30 & -5.37 & 5.06 & 0.41 & -0.11 \\
\hline
\end{tabular}


Asia Pacific Region : Tree Cover on Agricultural Land (2010)

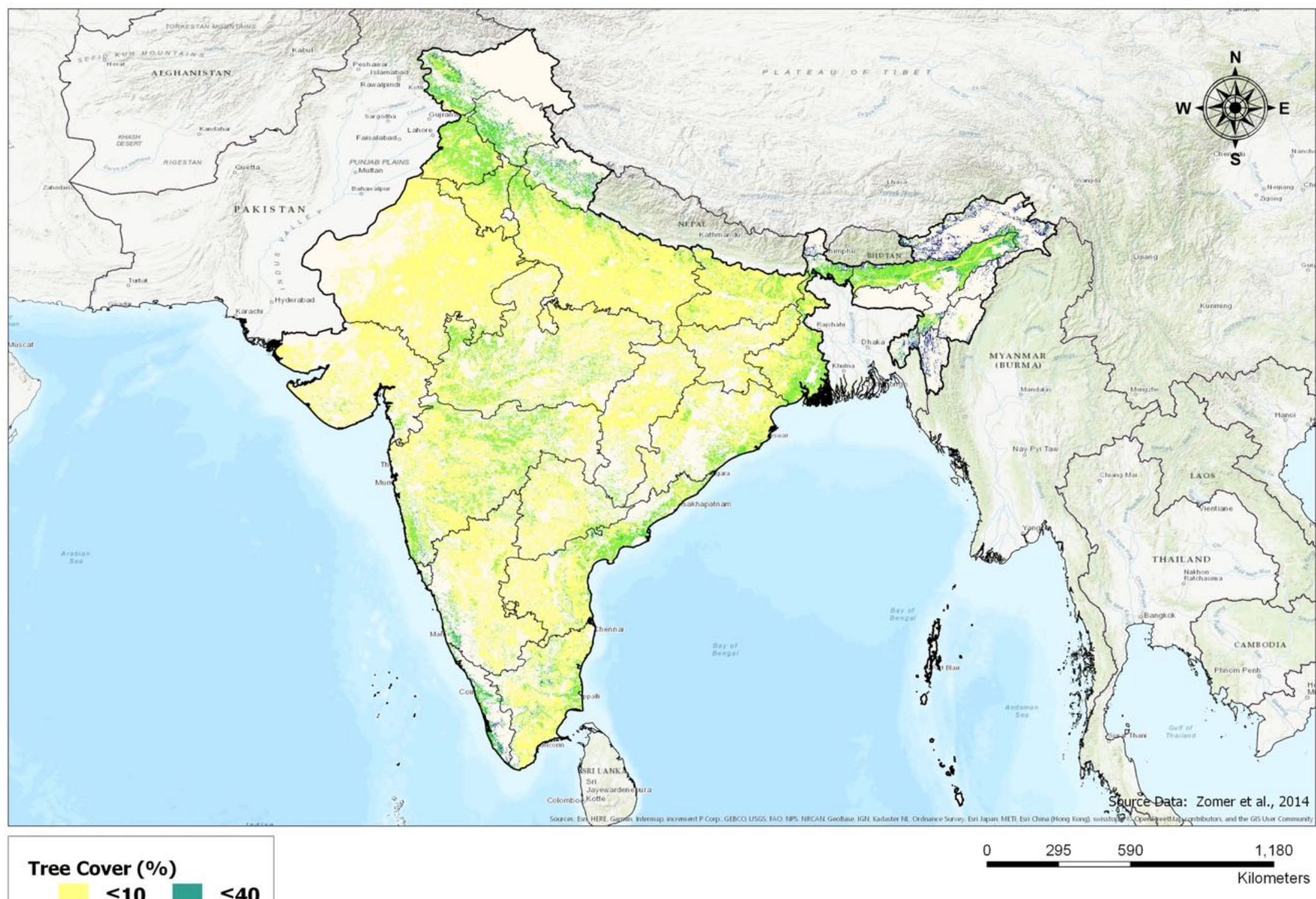

India 
Asia Pacific Region : Change in Tree Cover on Agricultural Land (2000 - 2010)

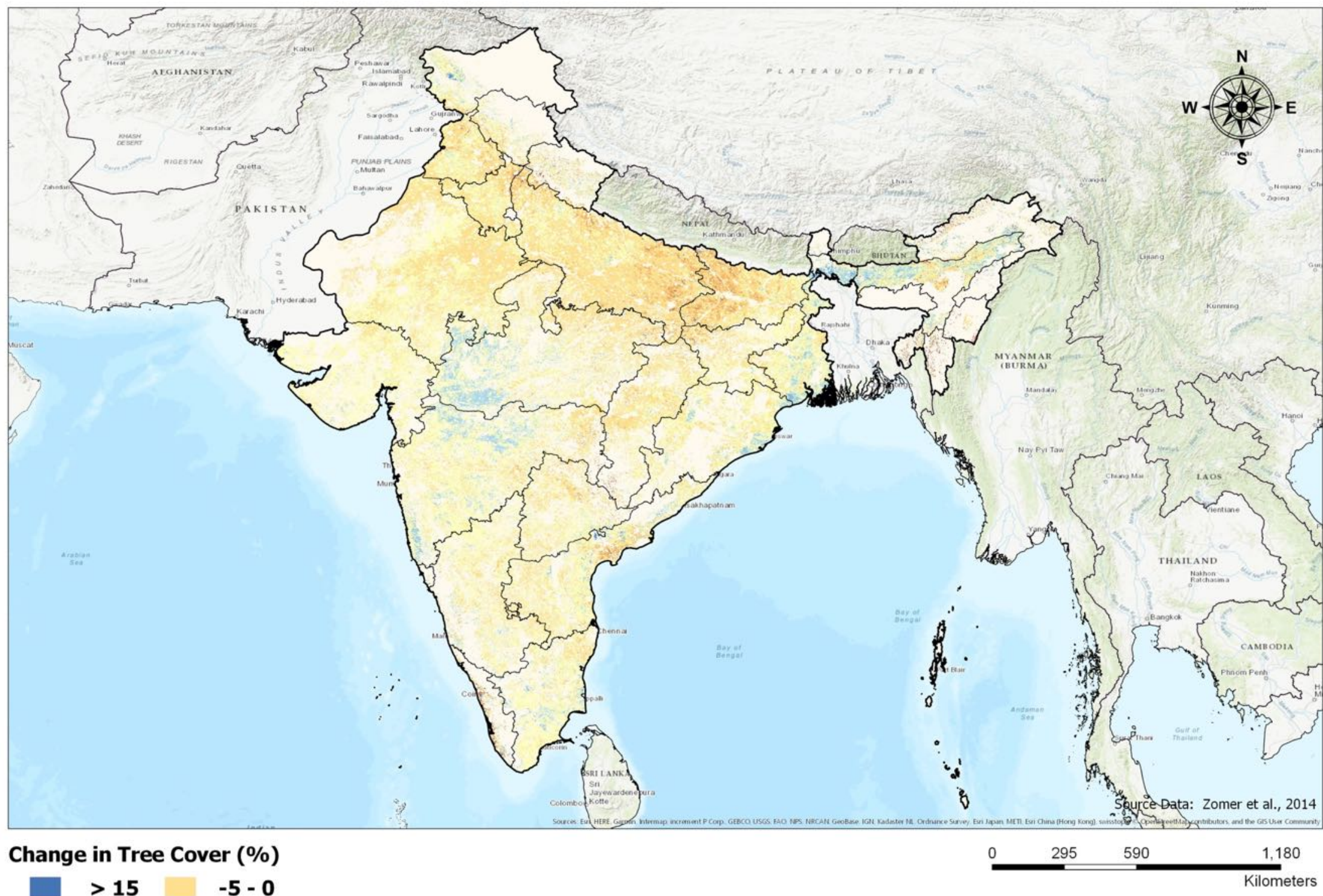

$10-15 \quad-10--5$

$5-10-15--10$

$0-5 \square \leq-15$

\section{India}




\section{Appendix 9: Indonesia}

Average Percent Tree Cover by Province, and Areal Extent of Tree Cover Classes $\left(\mathrm{km}^{2}\right)$

\begin{tabular}{|c|c|c|c|c|c|c|c|c|c|c|c|c|c|c|c|c|}
\hline \multicolumn{2}{|c|}{ Indonesia } & \multicolumn{3}{|c|}{ Average Tree Cover by Province } & \multicolumn{12}{|c|}{ Areal Extent of Tree Cover Class $\left(\mathrm{km}^{2}\right)$} \\
\hline \multirow{3}{*}{ Province } & \multirow{3}{*}{ Area_km } & \multirow{2}{*}{\multicolumn{3}{|c|}{$\begin{array}{c}\text { Mean Tree Cover Percent by Province } \\
(\%)\end{array}$}} & \multirow{2}{*}{\multicolumn{4}{|c|}{$\begin{array}{c}\text { Year } 2000 \\
\end{array}$}} & \multirow{2}{*}{\multicolumn{4}{|c|}{$\begin{array}{c}\text { Year } 2010 \\
\text { Area by Tree Cover Class }\left(\mathrm{km}^{2}\right)\end{array}$}} & \multirow{2}{*}{\multicolumn{4}{|c|}{$\begin{array}{l}\text { Change (2000 to 2010) } \\
\text { Are }\end{array}$}} \\
\hline & & & & & & & & & & & & & & & & \\
\hline & & 2000 & 2010 & Change & $<10 \%$ & $10-20 \%$ & $20-30 \%$ & $>30 \%$ & $<10 \%$ & $10-20 \%$ & $20-30 \%$ & $>30 \%$ & $<10 \%$ & $10-20 \%$ & $20-30 \%$ & $>30 \%$ \\
\hline Aceh & 17,479 & 40.52 & 43.26 & 2.74 & 349 & 2,348 & 2,954 & 11,828 & 115 & 2,305 & 2,300 & 12,759 & -234 & -43 & -654 & 931 \\
\hline Bali & 1,905 & 37.97 & 42.95 & 4.98 & 26 & 344 & 402 & 1,133 & 10 & 174 & 380 & 1,341 & -16 & -170 & -22 & 208 \\
\hline Bangka Belitung & 10,767 & 34.27 & 37.50 & 3.23 & 347 & 1,777 & 2,302 & 6,341 & 590 & 1,748 & 1,738 & 6,691 & 243 & -29 & -564 & 350 \\
\hline Banten & 4,891 & 33.89 & 34.06 & 0.17 & 637 & 867 & 632 & 2,755 & 254 & 1,302 & 697 & 2,638 & -383 & 435 & 65 & -117 \\
\hline Bengkulu & 9,303 & 45.01 & 50.28 & 5.27 & 13 & 545 & 1,258 & 7,487 & 3 & 157 & 659 & 8,484 & -10 & -388 & -599 & 997 \\
\hline Gorontalo & 3,901 & 39.13 & 40.23 & 1.10 & 64 & 751 & 761 & 2,325 & 5 & 451 & 1,053 & 2,392 & -59 & -300 & 292 & 67 \\
\hline Jambi & 25,019 & 42.11 & 46.35 & 4.23 & 104 & 2,423 & 3,783 & 18,709 & 57 & 1,482 & 3,066 & 20,414 & -47 & -941 & -717 & 1,705 \\
\hline Jawa Barat & 22,281 & 29.64 & 30.54 & 0.89 & 2,580 & 4,958 & 4,772 & 9,971 & 881 & 6,911 & 4,392 & 10,097 & $-1,699$ & 1,953 & -380 & 126 \\
\hline Jawa Tengah & 19,638 & 24.42 & 27.94 & 3.52 & 3,052 & 6,539 & 4,452 & 5,595 & 666 & 7,632 & 4,572 & 6,768 & $-2,386$ & 1,093 & 120 & 1,173 \\
\hline Jawa Timur & 25,498 & 23.31 & 27.02 & 3.70 & 4,409 & 9,252 & 5,717 & 6,120 & 1,850 & 8,398 & 7,384 & 7,866 & $-2,559$ & -854 & 1,667 & 1,746 \\
\hline Kalimantan Barat & 63,886 & 45.69 & 50.33 & 4.64 & 217 & 3,519 & 7,662 & 52,488 & 195 & 2,518 & 6,056 & 55,117 & -22 & $-1,001$ & $-1,606$ & 2,629 \\
\hline Kalimantan Selatan & 22,425 & 35.37 & 39.46 & 4.10 & 920 & 4,942 & 4,473 & 12,090 & 537 & 4,553 & 3,951 & 13,384 & -383 & -389 & -522 & 1,294 \\
\hline Kalimantan Tengah & 37,500 & 42.05 & 47.75 & 5.70 & 385 & 4,641 & 5,995 & 26,479 & 303 & 3,642 & 4,911 & 28,644 & -82 & -999 & $-1,084$ & 2,165 \\
\hline Kalimantan Timur & 25,559 & 44.70 & 48.19 & 3.49 & 263 & 2,537 & 3,422 & 19,337 & 425 & 2,396 & 3,000 & 19,738 & 162 & -141 & -422 & 401 \\
\hline Kepulauan Riau & 1,786 & 33.03 & 33.79 & 0.76 & 93 & 434 & 369 & 890 & 172 & 380 & 297 & 937 & 79 & -54 & -72 & 47 \\
\hline Lampung & 25,744 & 23.17 & 27.66 & 4.49 & 6,249 & 8,476 & 3,982 & 7,037 & 3,183 & 9,367 & 3,791 & 9,403 & $-3,066$ & 891 & -191 & 2,366 \\
\hline Maluku & 9,792 & 48.30 & 50.23 & 1.93 & 256 & 814 & 1,088 & 7,634 & 165 & 635 & 1,167 & 7,825 & -91 & -179 & 79 & 191 \\
\hline Maluku Utara & 2,186 & 58.77 & 58.94 & 0.17 & 4 & 39 & 53 & 2,090 & 3 & 23 & 92 & 2,068 & -1 & -16 & 39 & -22 \\
\hline Nusa Tenggara Barat & 8,596 & 31.40 & 34.73 & 3.32 & 1,298 & 2,359 & 1,214 & 3,725 & 399 & 2,549 & 1,420 & 4,228 & -899 & 190 & 206 & 503 \\
\hline Nusa Tenggara Timur & 21,539 & 30.54 & 34.39 & 3.86 & 2,962 & 4,665 & 4,305 & 9,607 & 1,921 & 3,911 & 4,019 & 11,688 & $-1,041$ & -754 & -286 & 2,081 \\
\hline Papua & 47,827 & 49.17 & 50.63 & 1.46 & 397 & 3,620 & 6,540 & 37,270 & 484 & 3,157 & 6,077 & 38,109 & 87 & -463 & -463 & 839 \\
\hline Papua Barat & 7,684 & 61.34 & 61.69 & 0.35 & 38 & 201 & 242 & 7,203 & 23 & 217 & 365 & 7,079 & -15 & 16 & 123 & -124 \\
\hline Riau & 41,402 & 41.45 & 46.32 & 4.87 & 295 & 4,672 & 7,258 & 29,177 & 223 & 2,766 & 5,558 & 32,855 & -72 & $-1,906$ & $-1,700$ & 3,678 \\
\hline Sulawesi Barat & 3,661 & 46.15 & 49.51 & 3.36 & 20 & 223 & 381 & 3,037 & 0 & 114 & 397 & 3,150 & -20 & -109 & 16 & 113 \\
\hline Sulawesi Selatan & 17,894 & 29.47 & 33.79 & 4.32 & 1,351 & 5,654 & 3,804 & 7,085 & 248 & 4,767 & 3,928 & 8,951 & $-1,103$ & -887 & 124 & 1,866 \\
\hline Sulawesi Tengah & 12,838 & 48.55 & 47.72 & -0.83 & 61 & 692 & 1,361 & 10,724 & 193 & 839 & 1,601 & 10,205 & 132 & 147 & 240 & -519 \\
\hline Sulawesi Tenggara & 10,407 & 38.17 & 43.31 & 5.14 & 264 & 1,698 & 1,889 & 6,556 & 215 & 1,163 & 1,341 & 7,688 & -49 & -535 & -548 & 1,132 \\
\hline Sulawesi Utara & 4,326 & 48.94 & 54.65 & 5.71 & 16 & 224 & 421 & 3,665 & 2 & 135 & 345 & 3,844 & -14 & -89 & -76 & 179 \\
\hline Sumatera Barat & 12,608 & 43.58 & 47.34 & 3.76 & 59 & 1,258 & 1,997 & 9,294 & 25 & 849 & 1,849 & 9,885 & -34 & -409 & -148 & 591 \\
\hline Sumatera Selatan & 63,093 & 38.13 & 44.70 & 6.57 & 1,222 & 8,521 & 12,106 & 41,244 & 361 & 6,666 & 8,230 & 47,836 & -861 & $-1,855$ & $-3,876$ & 6,592 \\
\hline Sumatera Utara & 39,226 & 41.42 & 45.23 & 3.81 & 1,519 & 5,837 & 5,253 & 26,617 & 215 & 4,791 & 5,734 & 28,486 & $-1,304$ & $-1,046$ & 481 & 1,869 \\
\hline Yogyakarta & 1,172 & 27.88 & 31.31 & 3.44 & 37 & 329 & 404 & 402 & 1 & 256 & 429 & 486 & -36 & -73 & 25 & 84 \\
\hline Indonesia - All & 621,833 & 39.19 & 43.09 & 3.89 & 29,507 & 95,160 & 101,253 & 395,918 & 13,724 & 86,255 & 90,800 & 431,059 & $-15,783$ & $-8,905$ & $-10,453$ & 35,141 \\
\hline
\end{tabular}


Average Percent Tree Cover by Province, and Areal Extent of Tree Cover Classes as a Percent of the Total Agricultural Area (\%)

\begin{tabular}{|c|c|c|c|c|c|c|c|c|c|c|c|c|c|c|c|c|}
\hline \multicolumn{2}{|l|}{ Indonesia } & \multirow{3}{*}{\multicolumn{3}{|c|}{$\begin{array}{c}\text { Average Tree Cover by Province } \\
\text { Percent Tree Cover } \\
(\%)\end{array}$}} & \multicolumn{12}{|c|}{ Areal Extent of Tree Cover Class as a Percent of Total Agricultural Area (\%) } \\
\hline \multirow{3}{*}{ Province } & \multirow{3}{*}{ Area_km } & & & & \multirow{2}{*}{\multicolumn{4}{|c|}{$\begin{array}{c}\text { Year } 2000 \\
\text { Percent of Total Agrciultural Area }(\%)\end{array}$}} & \multirow{2}{*}{\multicolumn{4}{|c|}{$\begin{array}{c}\text { Year } 2010 \\
\text { Percent of Total Agrciultural Area (\%) }\end{array}$}} & \multirow{2}{*}{\multicolumn{4}{|c|}{$\begin{array}{c}\text { Change }(2000 \text { to } 2010) \\
\text { Percent of Total Agrciultural Area }(\%)\end{array}$}} \\
\hline & & & & & & & & & & & & & & & & \\
\hline & & 2000 & 2010 & Change & $<10 \%$ & $10-20 \%$ & $20-30 \%$ & $>30 \%$ & $<10 \%$ & $10-20 \%$ & $20-30 \%$ & $>30 \%$ & $<10 \%$ & $10-20 \%$ & $20-30 \%$ & $>30 \%$ \\
\hline Aceh & 17,479 & 40.52 & 43.26 & 2.74 & 2.00 & 13.43 & 16.90 & 67.67 & 0.66 & 13.19 & 13.16 & 73.00 & -1.34 & -0.25 & -3.74 & 5.33 \\
\hline Bali & 1,905 & 37.97 & 42.95 & 4.98 & 1.36 & 18.06 & 21.10 & 59.48 & 0.52 & 9.13 & 19.95 & 70.39 & -0.84 & -8.92 & -1.15 & 10.92 \\
\hline Bangka Belitung & 10,767 & 34.27 & 37.50 & 3.23 & 3.22 & 16.50 & 21.38 & 58.89 & 5.48 & 16.23 & 16.14 & 62.14 & 2.26 & -0.27 & -5.24 & 3.25 \\
\hline Banten & 4,891 & 33.89 & 34.06 & 0.17 & 13.02 & 17.73 & 12.92 & 56.33 & 5.19 & 26.62 & 14.25 & 53.94 & -7.83 & 8.89 & 1.33 & -2.39 \\
\hline Bengkulu & 9,303 & 45.01 & 50.28 & 5.27 & 0.14 & 5.86 & 13.52 & 80.48 & 0.03 & 1.69 & 7.08 & 91.20 & -0.11 & -4.17 & -6.44 & 10.72 \\
\hline Gorontalo & 3,901 & 39.13 & 40.23 & 1.10 & 1.64 & 19.25 & 19.51 & 59.60 & 0.13 & 11.56 & 26.99 & 61.32 & -1.51 & -7.69 & 7.49 & 1.72 \\
\hline Jambi & 25,019 & 42.11 & 46.35 & 4.23 & 0.42 & 9.68 & 15.12 & 74.78 & 0.23 & 5.92 & 12.25 & 81.59 & -0.19 & -3.76 & -2.87 & 6.81 \\
\hline Jawa Barat & 22,281 & 29.64 & 30.54 & 0.89 & 11.58 & 22.25 & 21.42 & 44.75 & 3.95 & 31.02 & 19.71 & 45.32 & -7.63 & 8.77 & -1.71 & 0.57 \\
\hline Jawa Tengah & 19,638 & 24.42 & 27.94 & 3.52 & 15.54 & 33.30 & 22.67 & 28.49 & 3.39 & 38.86 & 23.28 & 34.46 & -12.15 & 5.57 & 0.61 & 5.97 \\
\hline Jawa Timur & 25,498 & 23.31 & 27.02 & 3.70 & 17.29 & 36.29 & 22.42 & 24.00 & 7.26 & 32.94 & 28.96 & 30.85 & -10.04 & -3.35 & 6.54 & 6.85 \\
\hline Kalimantan Barat & 63,886 & 45.69 & 50.33 & 4.64 & 0.34 & 5.51 & 11.99 & 82.16 & 0.31 & 3.94 & 9.48 & 86.27 & -0.03 & -1.57 & -2.51 & 4.12 \\
\hline Kalimantan Selatan & 22,425 & 35.37 & 39.46 & 4.10 & 4.10 & 22.04 & 19.95 & 53.91 & 2.39 & 20.30 & 17.62 & 59.68 & -1.71 & -1.73 & -2.33 & 5.77 \\
\hline Kalimantan Tengah & 37,500 & 42.05 & 47.75 & 5.70 & 1.03 & 12.38 & 15.99 & 70.61 & 0.81 & 9.71 & 13.10 & 76.38 & -0.22 & -2.66 & -2.89 & 5.77 \\
\hline Kalimantan Timur & 25,559 & 44.70 & 48.19 & 3.49 & 1.03 & 9.93 & 13.39 & 75.66 & 1.66 & 9.37 & 11.74 & 77.23 & 0.63 & -0.55 & -1.65 & 1.57 \\
\hline Kepulauan Riau & 1,786 & 33.03 & 33.79 & 0.76 & 5.21 & 24.30 & 20.66 & 49.83 & 9.63 & 21.28 & 16.63 & 52.46 & 4.42 & -3.02 & -4.03 & 2.63 \\
\hline Lampung & 25,744 & 23.17 & 27.66 & 4.49 & 24.27 & 32.92 & 15.47 & 27.33 & 12.36 & 36.39 & 14.73 & 36.53 & -11.91 & 3.46 & -0.74 & 9.19 \\
\hline Maluku & 9,792 & 48.30 & 50.23 & 1.93 & 2.61 & 8.31 & 11.11 & 77.96 & 1.69 & 6.48 & 11.92 & 79.91 & -0.93 & -1.83 & 0.81 & 1.95 \\
\hline Maluku Utara & 2,186 & 58.77 & 58.94 & 0.17 & 0.18 & 1.78 & 2.42 & 95.61 & 0.14 & 1.05 & 4.21 & 94.60 & -0.05 & -0.73 & 1.78 & -1.01 \\
\hline Nusa Tenggara Barat & 8,596 & 31.40 & 34.73 & 3.32 & 15.10 & 27.44 & 14.12 & 43.33 & 4.64 & 29.65 & 16.52 & 49.19 & -10.46 & 2.21 & 2.40 & 5.85 \\
\hline Nusa Tenggara Timur & 21,539 & 30.54 & 34.39 & 3.86 & 13.75 & 21.66 & 19.99 & 44.60 & 8.92 & 18.16 & 18.66 & 54.26 & -4.83 & -3.50 & -1.33 & 9.66 \\
\hline Papua & 47,827 & 49.17 & 50.63 & 1.46 & 0.83 & 7.57 & 13.67 & 77.93 & 1.01 & 6.60 & 12.71 & 79.68 & 0.18 & -0.97 & -0.97 & 1.75 \\
\hline Papua Barat & 7,684 & 61.34 & 61.69 & 0.35 & 0.49 & 2.62 & 3.15 & 93.74 & 0.30 & 2.82 & 4.75 & 92.13 & -0.20 & 0.21 & 1.60 & -1.61 \\
\hline Riau & 41,402 & 41.45 & 46.32 & 4.87 & 0.71 & 11.28 & 17.53 & 70.47 & 0.54 & 6.68 & 13.42 & 79.36 & -0.17 & -4.60 & -4.11 & 8.88 \\
\hline Sulawesi Barat & 3,661 & 46.15 & 49.51 & 3.36 & 0.55 & 6.09 & 10.41 & 82.96 & 0.00 & 3.11 & 10.84 & 86.04 & -0.55 & -2.98 & 0.44 & 3.09 \\
\hline Sulawesi Selatan & 17,894 & 29.47 & 33.79 & 4.32 & 7.55 & 31.60 & 21.26 & 39.59 & 1.39 & 26.64 & 21.95 & 50.02 & -6.16 & -4.96 & 0.69 & 10.43 \\
\hline Sulawesi Tengah & 12,838 & 48.55 & 47.72 & -0.83 & 0.48 & 5.39 & 10.60 & 83.53 & 1.50 & 6.54 & 12.47 & 79.49 & 1.03 & 1.15 & 1.87 & -4.04 \\
\hline Sulawesi Tenggara & 10,407 & 38.17 & 43.31 & 5.14 & 2.54 & 16.32 & 18.15 & 63.00 & 2.07 & 11.18 & 12.89 & 73.87 & -0.47 & -5.14 & -5.27 & 10.88 \\
\hline Sulawesi Utara & 4,326 & 48.94 & 54.65 & 5.71 & 0.37 & 5.18 & 9.73 & 84.72 & 0.05 & 3.12 & 7.98 & 88.86 & -0.32 & -2.06 & -1.76 & 4.14 \\
\hline Sumatera Barat & 12,608 & 43.58 & 47.34 & 3.76 & 0.47 & 9.98 & 15.84 & 73.72 & 0.20 & 6.73 & 14.67 & 78.40 & -0.27 & -3.24 & -1.17 & 4.69 \\
\hline Sumatera Selatan & 63,093 & 38.13 & 44.70 & 6.57 & 1.94 & 13.51 & 19.19 & 65.37 & 0.57 & 10.57 & 13.04 & 75.82 & -1.36 & -2.94 & -6.14 & 10.45 \\
\hline Sumatera Utara & 39,226 & 41.42 & 45.23 & 3.81 & 3.87 & 14.88 & 13.39 & 67.86 & 0.55 & 12.21 & 14.62 & 72.62 & -3.32 & -2.67 & 1.23 & 4.76 \\
\hline Yogyakarta & 1,172 & 27.88 & 31.31 & 3.44 & 3.16 & 28.07 & 34.47 & 34.30 & 0.09 & 21.84 & 36.60 & 41.47 & -3.07 & -6.23 & 2.13 & 7.17 \\
\hline Indonesia - All & 621,833 & 39.19 & 43.09 & 3.89 & 4.75 & 15.30 & 16.28 & 63.67 & 2.21 & 13.87 & 14.60 & 69.32 & -2.54 & -1.43 & -1.68 & 5.65 \\
\hline
\end{tabular}


Asia Pacific Region : Tree Cover on Agricultural Land (2010)

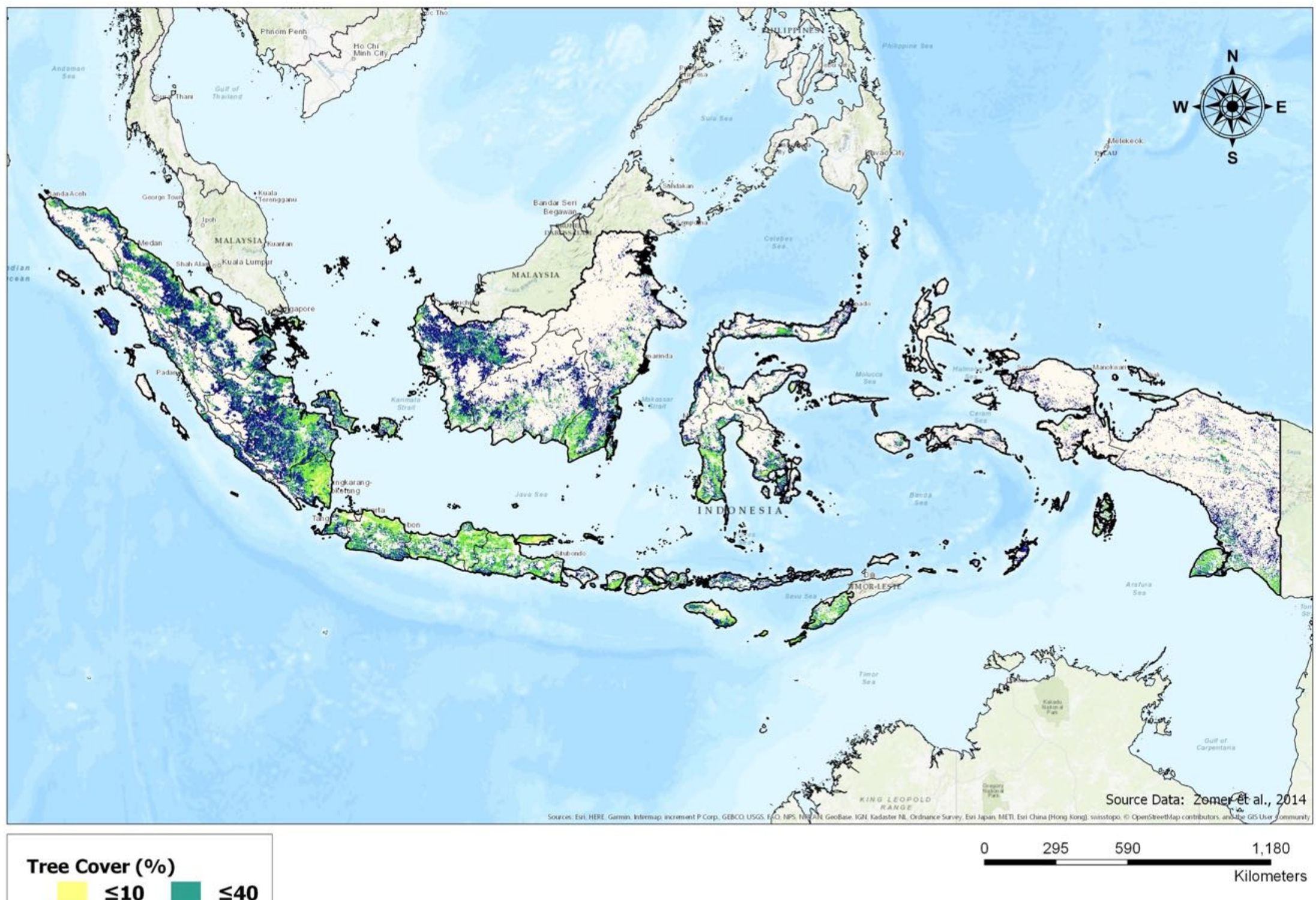

\section{Indonesia}


Asia Pacific Region : Change in Tree Cover on Agricultural Land (2000 - 2010)

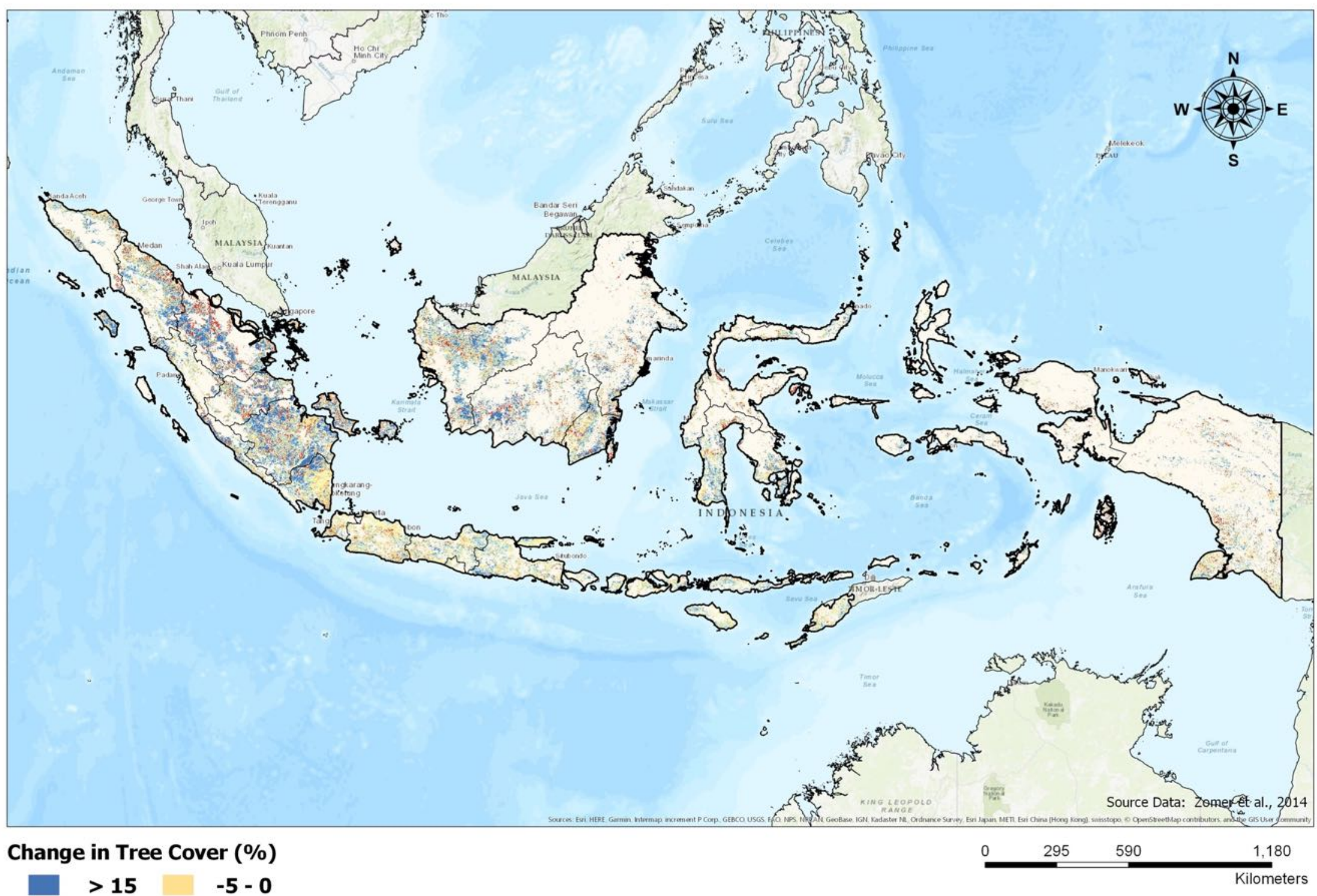

$>15$

$10-15 \quad-10--5$

$-10-15--10$

Indonesia

$0-5 \square \leq-15$ 


\section{Appendix 10: Japan}

Average Percent Tree Cover by Province, and Areal Extent of Tree Cover Classes $\left(\mathrm{km}^{2}\right)$

\begin{tabular}{|c|c|c|c|c|c|c|c|c|c|c|c|c|c|c|c|c|}
\hline \multicolumn{2}{|c|}{ Japan } & \multirow{2}{*}{\multicolumn{3}{|c|}{\begin{tabular}{|c|} 
Average Tree Cover by Province \\
\end{tabular}}} & \multicolumn{12}{|c|}{ Areal Extent of Tree Cover Class $\left(\mathrm{km}^{2}\right)$} \\
\hline \multirow{3}{*}{ Province } & \multirow{3}{*}{ Area_km } & & & & \multicolumn{4}{|c|}{ Year 2000 } & \multirow{2}{*}{\multicolumn{4}{|c|}{$\begin{array}{c}\text { Year } 2010 \\
\text { Area by Tree Cover Class }\left(\mathrm{km}^{2}\right)\end{array}$}} & \multirow{2}{*}{\multicolumn{4}{|c|}{$\begin{array}{l}\text { Change (2000 to 2010) } \\
\text { Area }\end{array}$}} \\
\hline & & \multicolumn{3}{|c|}{ Mean Tree Cover Percent by Province } & & a by Tree $C$ & ver Class & & & & & & & & & \\
\hline & & 2000 & 2010 & Change & $<10 \%$ & $10-20 \%$ & $20-30 \%$ & $>30 \%$ & $<10 \%$ & $10-20 \%$ & $20-30 \%$ & $>30 \%$ & $<10 \%$ & $10-20 \%$ & $20-30 \%$ & $>30 \%$ \\
\hline Aichi & 37 & 45.24 & 49.46 & 4.22 & 2 & 4 & 1 & 30 & 3 & 3 & 0 & 31 & 1 & -1 & -1 & 1 \\
\hline Akita & 1,278 & 44.33 & 41.00 & -3.33 & 23 & 263 & 165 & 827 & 33 & 292 & 146 & 807 & 10 & 29 & -19 & -20 \\
\hline Aomori & 1,295 & 56.08 & 54.58 & -1.50 & 20 & 83 & 96 & 1,096 & 10 & 89 & 102 & 1,094 & -10 & 6 & 6 & -2 \\
\hline Chiba & 484 & 25.78 & 27.95 & 2.16 & 2 & 174 & 179 & 129 & 0 & 147 & 146 & 191 & -2 & -27 & -33 & 62 \\
\hline Ehime & 549 & 48.16 & 51.83 & 3.67 & 6 & 33 & 51 & 459 & 8 & 23 & 37 & 481 & 2 & -10 & -14 & 22 \\
\hline Fukui & 162 & 41.62 & 44.83 & 3.20 & 5 & 25 & 18 & 114 & 3 & 24 & 18 & 117 & -2 & -1 & 0 & 3 \\
\hline Fukuoka & 466 & 36.51 & 40.74 & 4.24 & 20 & 102 & 70 & 274 & 25 & 71 & 64 & 306 & 5 & -31 & -6 & 32 \\
\hline Fukushima & 1,579 & 45.18 & 41.74 & -3.44 & 5 & 210 & 238 & 1,126 & 8 & 225 & 221 & 1,125 & 3 & 15 & -17 & -1 \\
\hline Gifu & 449 & 44.71 & 41.04 & -3.67 & 5 & 66 & 59 & 319 & 24 & 52 & 66 & 307 & 19 & -14 & 7 & -12 \\
\hline Gunma & 918 & 52.24 & 50.97 & -1.26 & 14 & 50 & 93 & 761 & 20 & 41 & 76 & 781 & 6 & -9 & -17 & 20 \\
\hline Hiroshima & 569 & 35.43 & 38.94 & 3.51 & 13 & 99 & 125 & 332 & 10 & 58 & 121 & 380 & -3 & -41 & -4 & 48 \\
\hline Hokkaido & 16,205 & 46.44 & 42.42 & -4.02 & 398 & 3,124 & 2,327 & 10,356 & 520 & 3,332 & 2,287 & 10,066 & 122 & 208 & -40 & -290 \\
\hline Hyōgo & 1,091 & 40.87 & 41.83 & 0.97 & 22 & 158 & 192 & 719 & 14 & 124 & 212 & 741 & -8 & -34 & 20 & 22 \\
\hline Ibaraki & 1,878 & 21.21 & 22.81 & 1.60 & 122 & 956 & 552 & 248 & 128 & 804 & 598 & 348 & 6 & -152 & 46 & 100 \\
\hline Ishikawa & 450 & 50.11 & 49.14 & -0.97 & 5 & 21 & 41 & 383 & 4 & 31 & 32 & 383 & -1 & 10 & -9 & 0 \\
\hline Iwate & 2,549 & 55.68 & 53.09 & -2.58 & 3 & 123 & 184 & 2,239 & 4 & 154 & 163 & 2,228 & 1 & 31 & -21 & -11 \\
\hline Kagawa & 69 & 35.46 & 39.55 & 4.09 & 6 & 12 & 12 & 39 & 4 & 8 & 13 & 44 & -2 & -4 & 1 & 5 \\
\hline Kagoshima & 1,201 & 40.37 & 44.00 & 3.63 & 53 & 157 & 244 & 747 & 11 & 119 & 222 & 849 & -42 & -38 & -22 & 102 \\
\hline Kanagawa & 31 & 54.90 & 65.39 & 10.48 & 1 & 1 & 0 & 29 & 1 & 1 & 0 & 29 & 0 & 0 & 0 & 0 \\
\hline Kochi & 174 & 43.70 & 45.86 & 2.16 & 7 & 11 & 17 & 139 & 6 & 10 & 14 & 144 & -1 & -1 & -3 & 5 \\
\hline Kumamoto & 621 & 34.52 & 37.49 & 2.97 & 12 & 143 & 134 & 332 & 27 & 100 & 101 & 393 & 15 & -43 & -33 & 61 \\
\hline Kyoto & 438 & 46.23 & 46.64 & 0.42 & 5 & 30 & 57 & 346 & 5 & 36 & 56 & 341 & 0 & 6 & -1 & -5 \\
\hline Mie & 337 & 37.74 & 42.09 & 4.35 & 6 & 71 & 57 & 203 & 0 & 25 & 84 & 228 & -6 & -46 & 27 & 25 \\
\hline Miyagi & 1,621 & 33.47 & 32.43 & -1.05 & 70 & 484 & 315 & 752 & 214 & 399 & 253 & 755 & 144 & -85 & -62 & 3 \\
\hline Miyazaki & 209 & 39.03 & 38.89 & -0.14 & 3 & 30 & 50 & 126 & 1 & 36 & 49 & 123 & -2 & 6 & -1 & -3 \\
\hline Nagano & 1,217 & 46.57 & 45.77 & -0.80 & 35 & 158 & 133 & 891 & 54 & 141 & 123 & 899 & 19 & -17 & -10 & 8 \\
\hline Naoasaki & 757 & 37.63 & 41.25 & 3.63 & 33 & 127 & 141 & 456 & 19 & 96 & 132 & 510 & -14 & -31 & -9 & 54 \\
\hline Nara & 141 & 44.79 & 50.07 & 5.28 & 0 & 10 & 14 & 117 & 0 & 3 & 11 & 127 & 0 & -7 & -3 & 10 \\
\hline Niigata & 2,383 & 51.28 & 45.97 & -5.32 & 28 & 191 & 181 & 1,983 & 43 & 222 & 268 & 1,850 & 15 & 31 & 87 & -133 \\
\hline Oita & 621 & 40.23 & 42.27 & 2.04 & 13 & 57 & 94 & 457 & 3 & 65 & 76 & 477 & -10 & 8 & -18 & 20 \\
\hline Okayama & 531 & 36.62 & 37.24 & 0.62 & 13 & 90 & 115 & 313 & 9 & 101 & 101 & 320 & -4 & 11 & -14 & 7 \\
\hline Okinawa & 247 & 22.61 & 25.57 & 2.97 & 59 & 94 & 33 & 61 & 43 & 91 & 47 & 66 & -16 & -3 & 14 & 5 \\
\hline Osaka & 54 & 47.54 & 50.70 & 3.17 & 0 & 3 & 6 & 45 & 0 & 1 & 6 & 47 & 0 & -2 & 0 & 2 \\
\hline Saga & $\begin{array}{r}549 \\
439\end{array}$ & 35.97 & 40.71 & 4.74 & 16 & 78 & 87 & 258 & 14 & 51 & 77 & 297 & -2 & -27 & -10 & 39 \\
\hline Saitama & 85 & 51.87 & 54.53 & 2.66 & 4 & 10 & 4 & 67 & 4 & 10 & 1 & 70 & 0 & 0 & -3 & 3 \\
\hline Shiga & 512 & 33.01 & 36.70 & 3.69 & 31 & 154 & 102 & 225 & 8 & 152 & 80 & 272 & -23 & -2 & -22 & 47 \\
\hline Shimane & 313 & 39.61 & 42.92 & 3.32 & 1 & 42 & 51 & 219 & 0 & 34 & 42 & 237 & -1 & -8 & -9 & 18 \\
\hline Shizuoka & 352 & 44.53 & 45.82 & 1.29 & 39 & 17 & 44 & 252 & 39 & 12 & 39 & 262 & 0 & -5 & -5 & 10 \\
\hline Tochigi & 1,938 & 38.69 & 38.43 & -0.26 & 65 & 399 & 298 & 1,176 & 92 & 408 & 327 & 1,111 & 27 & 9 & 29 & -65 \\
\hline Tokushima & 102 & 45.90 & 48.52 & 2.62 & 2 & 13 & 9 & 78 & 6 & 8 & 9 & 79 & 4 & -5 & 0 & 1 \\
\hline Tokyo & 6 & 63.33 & 67.67 & 4.33 & 0 & 0 & 0 & 6 & 0 & 0 & 0 & 6 & 0 & 0 & 0 & 0 \\
\hline Tottori & 438 & 35.32 & 34.16 & -1.16 & 27 & 101 & 71 & 239 & 19 & 116 & 79 & 224 & -8 & 15 & 8 & -15 \\
\hline Toyama & 900 & 40.66 & 35.82 & -4.84 & 55 & 145 & 101 & 599 & 93 & 131 & 131 & 545 & 38 & -14 & 30 & -54 \\
\hline Wakayama & 63 & 47.21 & 50.37 & 3.16 & 0 & 1 & 4 & 58 & 0 & 0 & 6 & 57 & 0 & -1 & 2 & -1 \\
\hline Yamagata & 1,305 & 47.97 & 40.61 & -7.36 & 7 & 184 & 140 & 974 & 31 & 205 & 185 & 884 & 24 & 21 & 45 & -90 \\
\hline Yamaguchi & 692 & 39.05 & 42.94 & $\begin{array}{r}-1.00 \\
3.89\end{array}$ & 27 & 87 & 110 & 468 & 14 & 78 & 80 & 520 & $\begin{array}{r}-13 \\
-13\end{array}$ & -9 & $\begin{array}{r}40 \\
-30\end{array}$ & 52 \\
\hline Yamanashi & 251 & 52.51 & 52.61 & 0.10 & 1 & 15 & 27 & 208 & 0 & 23 & 33 & 195 & -1 & 8 & 6 & -13 \\
\hline Japan - All & 48,010 & 43.76 & 42.07 & -1.70 & 1,284 & 8,406 & 7,044 & 31,276 & 1,574 & 8,152 & 6,935 & 31,349 & 290 & -254 & -109 & 73 \\
\hline
\end{tabular}


Average Percent Tree Cover by Province, and Areal Extent of Tree Cover Classes as a Percent of the Total Agricultural Area (\%)

\begin{tabular}{|c|c|c|c|c|c|c|c|c|c|c|c|c|c|c|c|c|}
\hline \multicolumn{2}{|c|}{ Japan } & \multirow{3}{*}{\multicolumn{3}{|c|}{$\begin{array}{c}\text { Average Tree Cover by Province } \\
\text { Percent Tree Cover }\end{array}$}} & \multicolumn{12}{|c|}{ Areal Extent of Tree Cover Class as a Percent of Total Agricultural Area (\%) } \\
\hline \multirow{3}{*}{ Province } & \multirow{3}{*}{ Area_km } & & & & \multirow{2}{*}{\multicolumn{4}{|c|}{$\begin{array}{c}\text { Year } 2000 \\
\end{array}$}} & \multirow{2}{*}{\multicolumn{4}{|c|}{$\begin{array}{c}\text { Year 2010 } \\
\text { Percent of Total Agrciultural Area }(\%) \\
\end{array}$}} & \multirow{2}{*}{\multicolumn{4}{|c|}{$\begin{array}{c}\text { Change (2000 to 2010) } \\
\text { Percent of Total Agrciultural Area (\%) }\end{array}$}} \\
\hline & & & & & & & & $(\%)$ & & & & & & & & \\
\hline & & 2000 & 2010 & Change & $<10 \%$ & $10-20 \%$ & $20-30 \%$ & $>30 \%$ & $<10 \%$ & $10-20 \%$ & $20-30 \%$ & $\frac{1}{>30 \%}$ & $<10 \%$ & $10-20 \%$ & $20-30 \%$ & $>30 \%$ \\
\hline Aichi & 37 & 45.24 & 49.46 & 4.22 & 5.41 & 10.81 & 2.70 & 81.08 & 8.11 & 8.11 & 0.00 & 83.78 & 2.70 & -2.70 & -2.70 & 2.70 \\
\hline Akita & 1,278 & 44.33 & 41.00 & -3.33 & 1.80 & 20.58 & 12.91 & 64.71 & 2.58 & 22.85 & 11.42 & 63.15 & 0.78 & 2.27 & -1.49 & -1.56 \\
\hline Aomori & 1,295 & 56.08 & 54.58 & -1.50 & 1.54 & 6.41 & 7.41 & 84.63 & 0.77 & 6.87 & 7.88 & 84.48 & -0.77 & 0.46 & 0.46 & -0.15 \\
\hline Chiba & 484 & 25.78 & 27.95 & 2.16 & 0.41 & 35.95 & 36.98 & 26.65 & 0.00 & 30.37 & 30.17 & 39.46 & -0.41 & -5.58 & -6.82 & 12.81 \\
\hline Ehime & 549 & 48.16 & 51.83 & 3.67 & 1.09 & 6.01 & 9.29 & 83.61 & 1.46 & 4.19 & 6.74 & 87.61 & 0.36 & -1.82 & -2.55 & 4.01 \\
\hline Fukui & 162 & 41.62 & 44.83 & 3.20 & 3.09 & 15.43 & 11.11 & 70.37 & 1.85 & 14.81 & 11.11 & 72.22 & -1.23 & -0.62 & 0.00 & 1.85 \\
\hline Fukuoka & 466 & 36.51 & 40.74 & 4.24 & 4.29 & 21.89 & 15.02 & 58.80 & 5.36 & 15.24 & 13.73 & 65.67 & 1.07 & -6.65 & -1.29 & 6.87 \\
\hline Fukushima & 1.579 & 45.18 & 41.74 & -3.44 & 0.32 & 13.30 & 15.07 & 71.31 & 0.51 & 14.25 & 14.00 & 71.25 & 0.19 & 0.95 & -1.08 & -0.06 \\
\hline Gifu & 449 & 44.71 & 41.04 & -3.67 & 1.11 & 14.70 & 13.14 & 71.05 & 5.35 & 11.58 & 14.70 & 68.37 & 4.23 & -3.12 & 1.56 & -2.67 \\
\hline Gunma & 918 & 52.24 & 50.97 & -1.26 & 1.53 & 5.45 & 10.13 & 82.90 & 2.18 & 4.47 & 8.28 & 85.08 & 0.65 & -0.98 & -1.85 & 2.18 \\
\hline Hiroshima & 569 & 35.43 & 38.94 & 3.51 & 2.28 & 17.40 & 21.97 & 58.35 & 1.76 & 10.19 & 21.27 & 66.78 & -0.53 & -7.21 & -0.70 & 8.44 \\
\hline Hokkaido & 16,205 & 46.44 & 42.42 & -4.02 & 2.46 & 19.28 & 14.36 & 63.91 & 3.21 & 20.56 & 14.11 & 62.12 & 0.75 & 1.28 & -0.25 & -1.79 \\
\hline Hyōgo & 1,091 & 40.87 & 41.83 & 0.97 & 2.02 & 14.48 & 17.60 & 65.90 & 1.28 & 11.37 & 19.43 & 67.92 & -0.73 & -3.12 & 1.83 & 2.02 \\
\hline Ibaraki & 1,878 & 21.21 & 22.81 & 1.60 & 6.50 & 50.91 & 29.39 & 13.21 & 6.82 & 42.81 & 31.84 & 18.53 & 0.32 & -8.09 & 2.45 & 5.32 \\
\hline Ishikawa & 450 & 50.11 & 49.14 & -0.97 & 1.11 & 4.67 & 9.11 & 85.11 & 0.89 & 6.89 & 7.11 & 85.11 & -0.22 & 2.22 & -2.00 & 0.00 \\
\hline Iwate & 2,549 & 55.68 & 53.09 & -2.58 & 0.12 & 4.83 & 7.22 & 87.84 & 0.16 & 6.04 & 6.39 & 87.41 & 0.04 & 1.22 & -0.82 & -0.43 \\
\hline Kagawa & 69 & 35.46 & 39.55 & 4.09 & 8.70 & 17.39 & 17.39 & 56.52 & 5.80 & 11.59 & 18.84 & 63.77 & -2.90 & -5.80 & 1.45 & 7.25 \\
\hline Kagoshima & 1.201 & 40.37 & 44.00 & 3.63 & 4.41 & 13.07 & 20.32 & 62.20 & 0.92 & 9.91 & 18.48 & 70.69 & -3.50 & -3.16 & -1.83 & 8.49 \\
\hline Kanagawa & 31 & 54.90 & 65.39 & 10.48 & 3.23 & 3.23 & 0.00 & 93.55 & 3.23 & 3.23 & 0.00 & 93.55 & 0.00 & 0.00 & 0.00 & 0.00 \\
\hline Kochi & 174 & 43.70 & 45.86 & 2.16 & 4.02 & 6.32 & 9.77 & 79.89 & 3.45 & 5.75 & 8.05 & 82.76 & -0.57 & -0.57 & -1.72 & 2.87 \\
\hline Kumamoto & 621 & 34.52 & 37.49 & 2.97 & 1.93 & 23.03 & 21.58 & 53.46 & 4.35 & 16.10 & 16.26 & 63.29 & 2.42 & -6.92 & -5.31 & 9.82 \\
\hline Kyoto & 438 & 46.23 & 46.64 & 0.42 & 1.14 & 6.85 & 13.01 & 79.00 & 1.14 & 8.22 & 12.79 & 77.85 & 0.00 & 1.37 & -0.23 & -1.14 \\
\hline Mie & 337 & 37.74 & 42.09 & 4.35 & 1.78 & 21.07 & 16.91 & 60.24 & 0.00 & 7.42 & 24.93 & 67.66 & -1.78 & -13.65 & 8.01 & 7.42 \\
\hline Miyagi & 1,621 & 33.47 & 32.43 & -1.05 & 4.32 & 29.86 & 19.43 & 46.39 & 13.20 & 24.61 & 15.61 & 46.58 & 8.88 & -5.24 & -3.82 & 0.19 \\
\hline Miyazaki & 209 & 39.03 & 38.89 & -0.14 & 1.44 & 14.35 & 23.92 & 60.29 & 0.48 & 17.23 & 23.45 & 58.85 & -0.96 & 2.87 & -0.48 & -1.44 \\
\hline Nagano & 1,217 & 46.57 & 45.77 & -0.80 & 2.88 & 12.98 & 10.93 & 73.21 & 4.44 & 11.59 & 10.11 & 73.87 & 1.56 & -1.40 & -0.82 & 0.66 \\
\hline Naoasaki & 757 & 37.63 & 41.25 & 3.63 & 4.36 & 16.78 & 18.63 & 60.24 & 2.51 & 12.68 & 17.44 & 67.37 & -1.85 & -4.10 & -1.19 & 7.13 \\
\hline Nara & 141 & 44.79 & 50.07 & 5.28 & 0.00 & 7.09 & 9.93 & 82.98 & 0.00 & 2.13 & 7.80 & 90.07 & 0.00 & -4.96 & -2.13 & 7.09 \\
\hline Nigata & 2,383 & 51.28 & 45.97 & -5.32 & 1.17 & 8.02 & 7.60 & 83.22 & 1.80 & 9.32 & 11.25 & 77.63 & 0.63 & 1.30 & 3.65 & -5.58 \\
\hline Oita & 621 & 40.23 & 42.27 & 2.04 & 2.09 & 9.18 & 15.14 & 73.59 & 0.48 & 10.47 & 12.24 & 76.81 & -1.61 & 1.29 & -2.90 & 3.22 \\
\hline Okayama & 531 & 36.62 & 37.24 & 0.62 & 2.45 & 16.95 & 21.66 & 58.95 & 1.69 & 19.02 & 19.02 & 60.26 & -0.75 & 2.07 & -2.64 & 1.32 \\
\hline Okinawa & 247 & 22.61 & 25.57 & 2.97 & 23.89 & 38.06 & 13.36 & 24.70 & 17.41 & 36.84 & 19.03 & 26.72 & -6.48 & -1.21 & 5.67 & 2.02 \\
\hline Osaka & 54 & 47.54 & 50.70 & 3.17 & 0.00 & 5.56 & 11.11 & 83.33 & 0.00 & 1.85 & 11.11 & 87.04 & 0.00 & -3.70 & 0.00 & 3.70 \\
\hline Saga & 439 & 35.97 & 40.71 & 4.74 & 3.64 & 17.77 & 19.82 & 58.77 & 3.19 & 11.62 & 17.54 & 67.65 & -0.46 & -6.15 & -2.28 & 8.88 \\
\hline Saitama & 85 & 51.87 & 54.53 & 2.66 & 4.71 & 11.76 & 4.71 & 78.82 & 4.71 & 11.76 & 1.18 & 82.35 & 0.00 & 0.00 & -3.53 & 3.53 \\
\hline Shiga & 512 & 33.01 & 36.70 & 3.69 & 6.05 & 30.08 & 19.92 & 43.95 & 1.56 & 29.69 & 15.63 & 53.13 & -4.49 & -0.39 & -4.30 & 9.18 \\
\hline Shimane & 313 & 39.61 & 42.92 & 3.32 & 0.32 & 13.42 & 16.29 & 69.97 & 0.00 & 10.86 & 13.42 & 75.72 & -0.32 & -2.56 & -2.88 & 5.75 \\
\hline Shizuoka & 352 & 44.53 & 45.82 & 1.29 & 11.08 & 4.83 & 12.50 & 71.59 & 11.08 & 3.41 & 11.08 & 74.43 & 0.00 & -1.42 & -1.42 & 2.84 \\
\hline Tochigi & 1,938 & 38.69 & 38.43 & -0.26 & 3.35 & 20.59 & 15.38 & 60.68 & 4.75 & 21.05 & 16.87 & 57.33 & 1.39 & 0.46 & 1.50 & -3.35 \\
\hline Tokushima & 102 & 45.90 & 48.52 & 2.62 & 1.96 & 12.75 & 8.82 & 76.47 & 5.88 & 7.84 & 8.82 & 77.45 & 3.92 & -4.90 & 0.00 & 0.98 \\
\hline Tokyo & 6 & 63.33 & 67.67 & 4.33 & 0.00 & 0.00 & 0.00 & 100.00 & 0.00 & 0.00 & 0.00 & 100.00 & 0.00 & 0.00 & 0.00 & 0.00 \\
\hline Tottori & 438 & 35.32 & 34.16 & -1.16 & 6.16 & 23.06 & 16.21 & 54.57 & 4.34 & 26.48 & 18.04 & 51.14 & -1.83 & 3.42 & 1.83 & -3.42 \\
\hline Toyama & 900 & 40.66 & 35.82 & -4.84 & 6.11 & 16.11 & 11.22 & 66.56 & 10.33 & 14.56 & 14.56 & 60.56 & 4.22 & -1.56 & 3.33 & -6.00 \\
\hline Wakayama & 63 & 47.21 & 50.37 & 3.16 & 0.00 & 1.59 & 6.35 & 92.06 & 0.00 & 0.00 & 9.52 & 90.48 & 0.00 & -1.59 & 3.17 & -1.59 \\
\hline Yamagata & 1,305 & 47.97 & 40.61 & -7.36 & 0.54 & 14.10 & 10.73 & 74.64 & 2.38 & 15.71 & 14.18 & 67.74 & 1.84 & 1.61 & 3.45 & -6.90 \\
\hline Yamaguchi & 692 & 39.05 & 42.94 & 3.89 & 3.90 & 12.57 & 15.90 & 67.63 & 2.02 & 11.27 & 11.56 & 75.15 & -1.88 & -1.30 & -4.34 & 7.51 \\
\hline Yamanashi & 251 & 52.51 & 52.61 & 0.10 & 0.40 & 5.98 & 10.76 & 82.87 & 0.00 & 9.16 & 13.15 & 77.69 & -0.40 & 3.19 & 2.39 & -5.18 \\
\hline Japan - All & 48,010 & 43.76 & 42.07 & -1.70 & 2.67 & 17.51 & 14.67 & 65.15 & 3.28 & 16.98 & 14.45 & 65.30 & 0.60 & -0.53 & -0.23 & 0.15 \\
\hline
\end{tabular}


Asia Pacific Region : Tree Cover on Agricultural Land (2010)

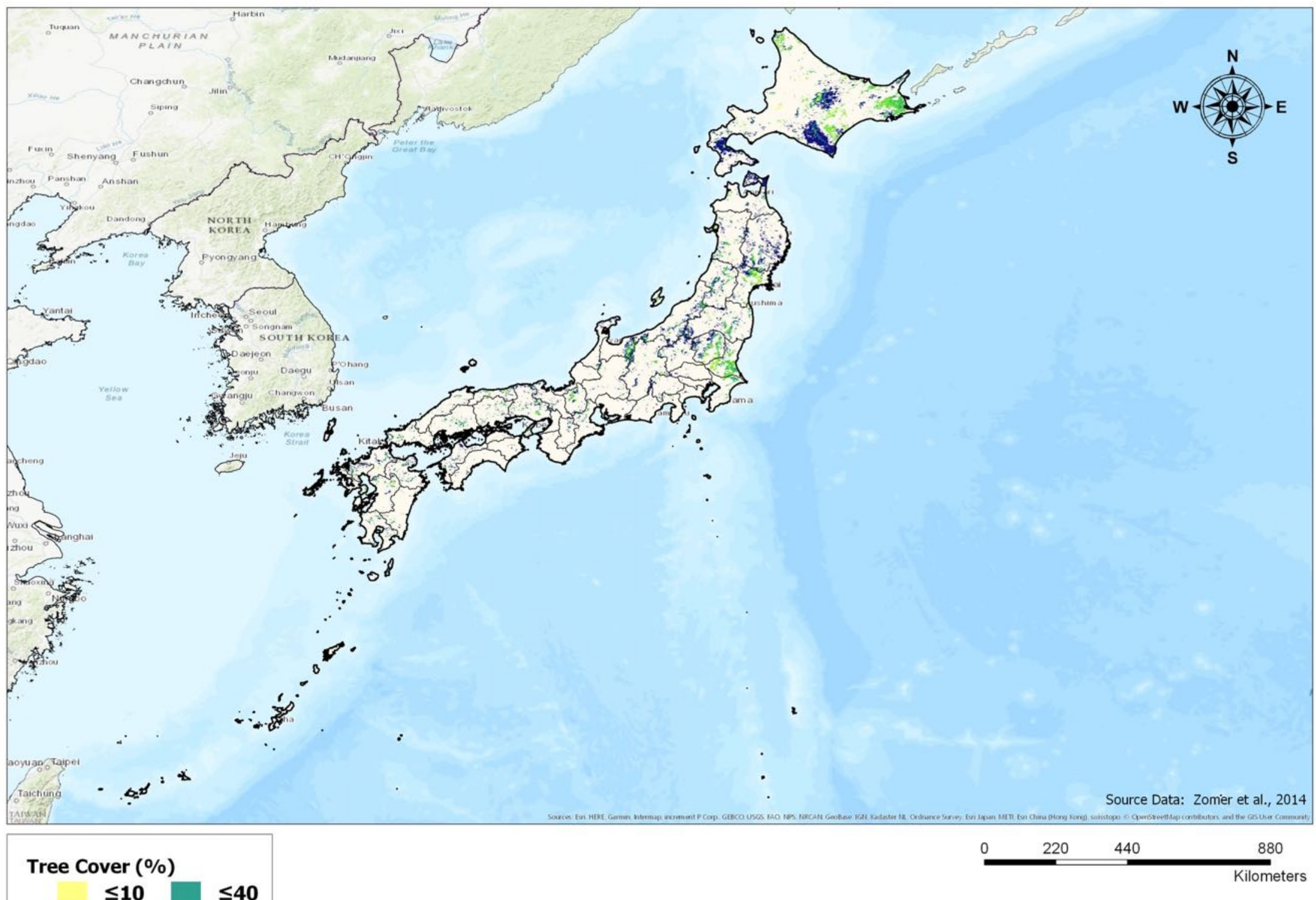

Japan 
Asia Pacific Region : Change in Tree Cover on Agricultural Land (2000 - 2010)

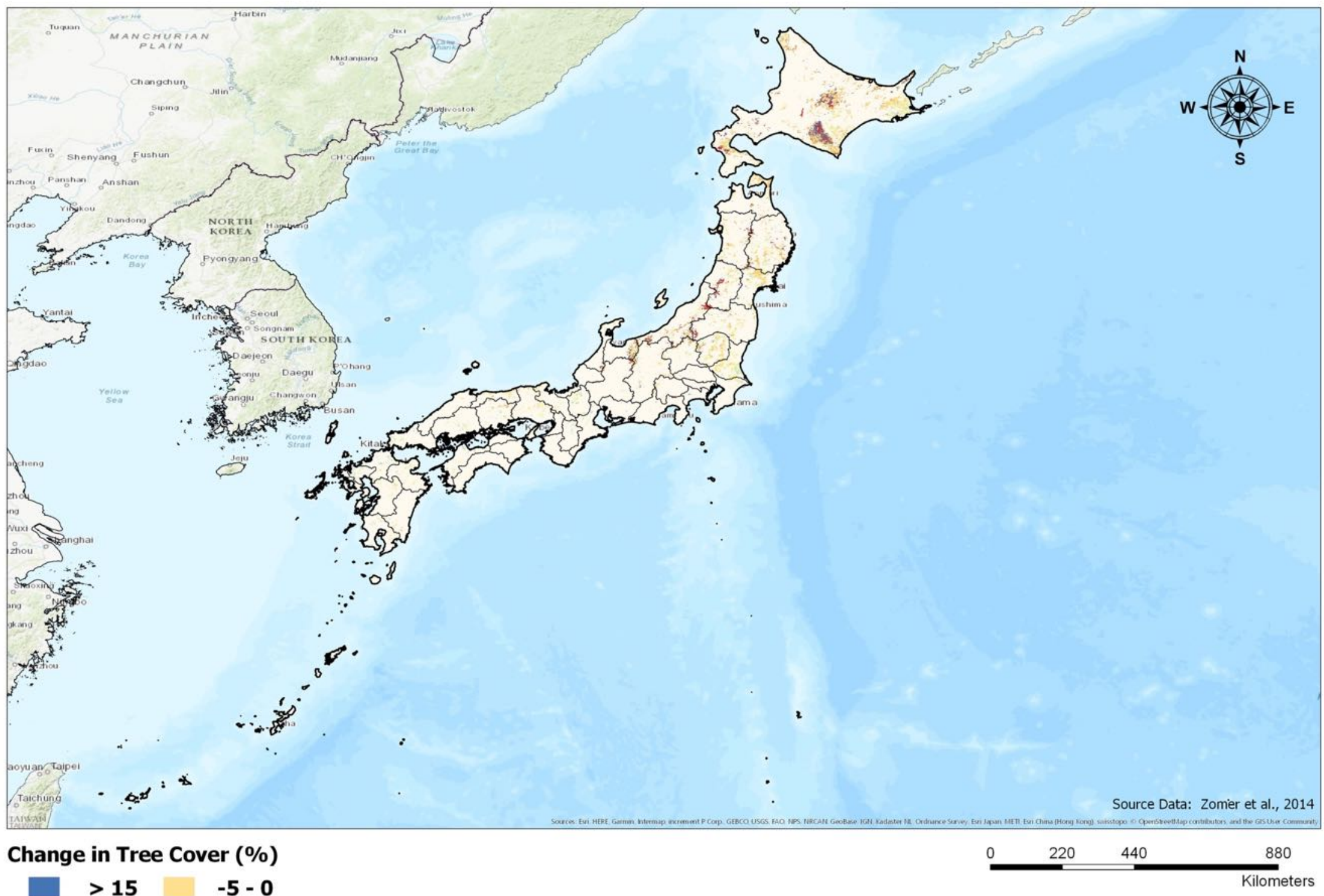

\begin{tabular}{|r|r|}
\hline$>15$ & $-5-0$ \\
$10-15$ & $-10--5$ \\
$5-10$ & $-15--10$ \\
$0-5$ & $\leq-15$
\end{tabular}

\section{Japan}


Average Percent Tree Cover by Province, and Areal Extent of Tree Cover Classes $\left(\mathrm{km}^{2}\right)$

\begin{tabular}{|c|c|c|c|c|c|c|c|c|c|c|c|c|c|c|c|c|}
\hline \multicolumn{2}{|l|}{ Laos } & \multirow{2}{*}{\multicolumn{3}{|c|}{\begin{tabular}{|c|} 
Average Tree Cover by Province \\
Mean Tree Cover Percent by Province
\end{tabular}}} & \multicolumn{12}{|c|}{ Areal Extent of Tree Cover Class $\left(\mathrm{km}^{2}\right)$} \\
\hline \multirow{3}{*}{ Province } & \multirow{3}{*}{ Area_km } & & & & \multicolumn{4}{|c|}{ Year 2000} & \multirow{2}{*}{\multicolumn{4}{|c|}{ Year 2010}} & \multicolumn{4}{|c|}{ Change (2000 to 2010) } \\
\hline & & & $(\%)$ & & & ea by Tree $\mathrm{C}$ & ver Class & & & & & & & by Tree C & er Class (k & \\
\hline & & 2000 & 2010 & Change & $<10 \%$ & $10-20 \%$ & $20-30 \%$ & $>30 \%$ & $<10 \%$ & $10-20 \%$ & $20-30 \%$ & $>30 \%$ & $<10 \%$ & $10-20 \%$ & $20-30 \%$ & $>\mathbf{3 0 \%}$ \\
\hline Attapu & 704 & 39.83 & 39.90 & 0.07 & 47 & 46 & 98 & 513 & 37 & 75 & 121 & 471 & -10 & 29 & 23 & -42 \\
\hline Bokeo & 2,282 & 53.78 & 46.13 & -7.65 & 34 & 84 & 101 & 2,063 & 102 & 131 & 211 & 1,838 & 68 & 47 & 110 & -225 \\
\hline Bolikhamxai & 2,598 & 45.59 & 43.04 & -2.55 & 147 & 214 & 189 & 2,048 & 97 & 261 & 346 & 1,894 & -50 & 47 & 157 & -154 \\
\hline Champasak & 2,816 & 20.76 & 19.35 & -1.41 & 1,396 & 280 & 223 & 917 & 1,315 & 458 & 300 & 743 & -81 & 178 & 77 & -174 \\
\hline Houaphan & 3,495 & 60.56 & 58.44 & -2.13 & 1 & 16 & 62 & 3,416 & 0 & 31 & 126 & 3,338 & -1 & 15 & 64 & -78 \\
\hline Khammouan & 1,856 & 35.51 & 33.64 & -1.86 & 370 & 170 & 193 & 1,123 & 363 & 219 & 215 & 1,059 & -7 & 49 & 22 & -64 \\
\hline Louang Namtha & 1,002 & 56.89 & 52.09 & -4.80 & 4 & 11 & 28 & 959 & 3 & 17 & 57 & 925 & -1 & 6 & 29 & -34 \\
\hline Louangphrabang & 5,646 & 57.26 & 56.27 & -1.00 & 3 & 25 & 95 & 5,523 & 4 & 59 & 176 & 5,407 & 1 & 34 & 81 & -116 \\
\hline Oudômxai & 2,667 & 55.67 & 51.77 & -3.90 & 2 & 21 & 42 & 2,602 & 1 & 28 & 118 & 2,520 & -1 & 7 & 76 & -82 \\
\hline Phôngsali & 2,700 & 54.68 & 57.55 & 2.86 & 0 & 2 & 75 & 2,623 & 0 & 8 & 87 & 2,605 & 0 & 6 & 12 & -18 \\
\hline Saravan & 1,692 & 34.30 & 33.83 & -0.47 & 318 & 144 & 150 & 1,080 & 276 & 143 & 231 & 1,042 & -42 & -1 & 81 & -38 \\
\hline Savannakhét & 5,683 & 31.16 & 32.46 & 1.30 & 1,453 & 573 & 556 & 3,101 & 1,282 & 558 & 701 & 3,142 & -171 & -15 & 145 & 41 \\
\hline Vientiane & 3,763 & 45.93 & 39.46 & -6.47 & 255 & 327 & 373 & 2,808 & 204 & 414 & 616 & 2,529 & -51 & 87 & 243 & -279 \\
\hline Vientiane [prefecture] & 2,006 & 29.45 & 27.31 & -2.14 & 474 & 373 & 258 & 901 & 378 & 441 & 371 & 816 & -96 & 68 & 113 & -85 \\
\hline Xaignabouri & 3,905 & 54.22 & 47.24 & -6.98 & 92 & 111 & 154 & 3,548 & 116 & 190 & 304 & 3,295 & 24 & 79 & 150 & -253 \\
\hline Xaisômboun & 945 & 54.37 & 51.74 & -2.62 & 1 & 14 & 35 & 895 & 1 & 18 & 72 & 854 & 0 & 4 & 37 & -41 \\
\hline Xékong & 423 & 45.61 & 41.50 & -4.12 & 0 & 9 & 21 & 393 & 4 & 38 & 79 & 302 & 4 & 29 & 58 & -91 \\
\hline Xiangkhoang & 1,781 & 51.03 & 52.51 & 1.48 & 103 & 63 & 77 & 1,538 & 120 & 43 & 89 & 1,529 & 17 & -20 & 12 & -9 \\
\hline Laos - All & 45,963 & 46.17 & 43.93 & -2.24 & 4,699 & 2,483 & 2,730 & 36,051 & 4,303 & 3,131 & 4,220 & 34,309 & -396 & 648 & 1,490 & $-1,742$ \\
\hline
\end{tabular}


Average Percent Tree Cover by Province, and Areal Extent of Tree Cover Classes as a Percent of the Total Agricultural Area (\%)

\begin{tabular}{|c|c|c|c|c|c|c|c|c|c|c|c|c|c|c|c|c|}
\hline \multicolumn{2}{|l|}{ Laos } & \multirow{2}{*}{\multicolumn{3}{|c|}{$\begin{array}{l}\text { Average Tree Cover by Province } \\
\text { Percent Tree Cover } \\
(\%)\end{array}$}} & \multicolumn{12}{|c|}{ Areal Extent of Tree Cover Class as a Percent of Total Agricultural Area (\%) } \\
\hline \multirow{2}{*}{ Province } & \multirow{2}{*}{ Area_km } & & & & \multicolumn{4}{|c|}{$\begin{array}{c}\text { Year 2000 } \\
\text { Percent of Total Agrciultural Area (\%) }\end{array}$} & \multirow{2}{*}{\multicolumn{4}{|c|}{$\begin{array}{c}\text { Year } 2010 \\
\text { Percent of Total Agrciultural Area (\%) }\end{array}$}} & \multirow{2}{*}{\multicolumn{4}{|c|}{ Percent of Total Agrciultural Area (\%) }} \\
\hline & & 2000 & $\frac{(10)}{2010}$ & Change & $<10 \%$ & $10-20 \%$ & $\frac{14 \text { tural } \mathrm{Ar}}{20-30 \%}$ & $\frac{(\%)}{>30 \%}$ & $<10 \%$ & & & & Per & & & \\
\hline Attapu & 704 & 39.83 & 39.90 & 0.07 & 6.68 & 6.53 & 13.92 & 72.87 & 5.26 & 10.65 & 17.19 & 66.90 & -1.42 & 4.12 & $\frac{20-30 \%}{327}$ & $>30 \%$ \\
\hline Bokeo & 2,282 & 53.78 & 46.13 & -7.65 & 1.49 & 3.68 & 4.43 & 90.40 & 4.47 & 5.74 & 9.25 & 80.54 & 2.98 & 2.06 & 4.82 & $\begin{array}{l}-5.91 \\
-9.86\end{array}$ \\
\hline Bolikhamxai & 2,598 & 45.59 & 43.04 & -2.55 & 5.66 & 8.24 & 7.27 & 78.83 & 3.73 & 10.05 & 13.32 & 72.90 & -1.92 & 1.81 & 6.04 & -5.93 \\
\hline Champasak & 2,816 & 20.76 & 19.35 & -1.41 & 49.57 & 9.94 & 7.92 & 32.56 & 46.70 & 16.26 & 10.65 & 26.39 & -2.88 & 6.32 & 2.73 & -6.18 \\
\hline Houaphan & 3,495 & 60.56 & 58.44 & -2.13 & 0.03 & 0.46 & 1.77 & 97.74 & 0.00 & 0.89 & 3.61 & 95.51 & -0.03 & 0.43 & 1.83 & -2.23 \\
\hline Khammouan & 1,856 & 35.51 & 33.64 & -1.86 & 19.94 & 9.16 & 10.40 & 60.51 & 19.56 & 11.80 & 11.58 & 57.06 & -0.38 & 2.64 & 1.19 & -3.45 \\
\hline Louang Namtha & 1,002 & 56.89 & 52.09 & -4.80 & 0.40 & 1.10 & 2.79 & 95.71 & 0.30 & 1.70 & 5.69 & 92.32 & -0.10 & 0.60 & 2.89 & -3.39 \\
\hline Louangphrabang & 5,646 & 57.26 & 56.27 & -1.00 & 0.05 & 0.44 & 1.68 & 97.82 & 0.07 & 1.04 & 3.12 & 95.77 & 0.02 & 0.60 & 1.43 & -2.05 \\
\hline Oudômxai & 2,667 & 55.67 & 51.77 & -3.90 & 0.07 & 0.79 & 1.57 & 97.56 & 0.04 & 1.05 & 4.42 & 94.49 & -0.04 & 0.26 & 2.85 & -3.07 \\
\hline Phôngsali & 2,700 & 54.68 & 57.55 & 2.86 & 0.00 & 0.07 & 2.78 & 97.15 & 0.00 & 0.30 & 3.22 & 96.48 & 0.00 & 0.22 & 0.44 & -0.67 \\
\hline Saravan & 1,692 & 34.30 & 33.83 & -0.47 & 18.79 & 8.51 & 8.87 & 63.83 & 16.31 & 8.45 & 13.65 & 61.58 & -2.48 & -0.06 & 4.79 & -2.25 \\
\hline Savannakhét & 5,683 & 31.16 & 32.46 & 1.30 & 25.57 & 10.08 & 9.78 & 54.57 & 22.56 & 9.82 & 12.34 & 55.29 & -3.01 & -0.26 & 2.55 & 0.72 \\
\hline Vientiane & 3,763 & 45.93 & 39.46 & -6.47 & 6.78 & 8.69 & 9.91 & 74.62 & 5.42 & 11.00 & 16.37 & 67.21 & -1.36 & 2.31 & 6.46 & -7.41 \\
\hline Vientiane [prefecture] & 2,006 & 29.45 & 27.31 & -2.14 & 23.63 & 18.59 & 12.86 & 44.92 & 18.84 & 21.98 & 18.49 & 40.68 & -4.79 & 3.39 & 5.63 & -4.24 \\
\hline Xaignabouri & 3,905 & 54.22 & 47.24 & -6.98 & 2.36 & 2.84 & 3.94 & 90.86 & 2.97 & 4.87 & 7.78 & 84.38 & 0.61 & 2.02 & 3.84 & -6.48 \\
\hline Xaisômboun & 945 & 54.37 & 51.74 & -2.62 & 0.11 & 1.48 & 3.70 & 94.71 & 0.11 & 1.90 & 7.62 & 90.37 & 0.00 & 0.42 & 3.92 & -4.34 \\
\hline Xékong & 423 & 45.61 & 41.50 & -4.12 & 0.00 & 2.13 & 4.96 & 92.91 & 0.95 & 8.98 & 18.68 & 71.40 & 0.95 & 6.86 & 13.71 & -21.51 \\
\hline Xiangkhoang & 1,781 & 51.03 & 52.51 & 1.48 & 5.78 & 3.54 & 4.32 & 86.36 & 6.74 & 2.41 & 5.00 & 85.85 & 0.95 & -1.12 & 0.67 & -0.51 \\
\hline Laos - All & 45,963 & 46.17 & 43.93 & -2.24 & 10.22 & 5.40 & 5.94 & 78.44 & 9.36 & 6.81 & 9.18 & 74.65 & -0.86 & 1.41 & 3.24 & -3.79 \\
\hline
\end{tabular}


Asia Pacific Region : Tree Cover on Agricultural Land (2010)

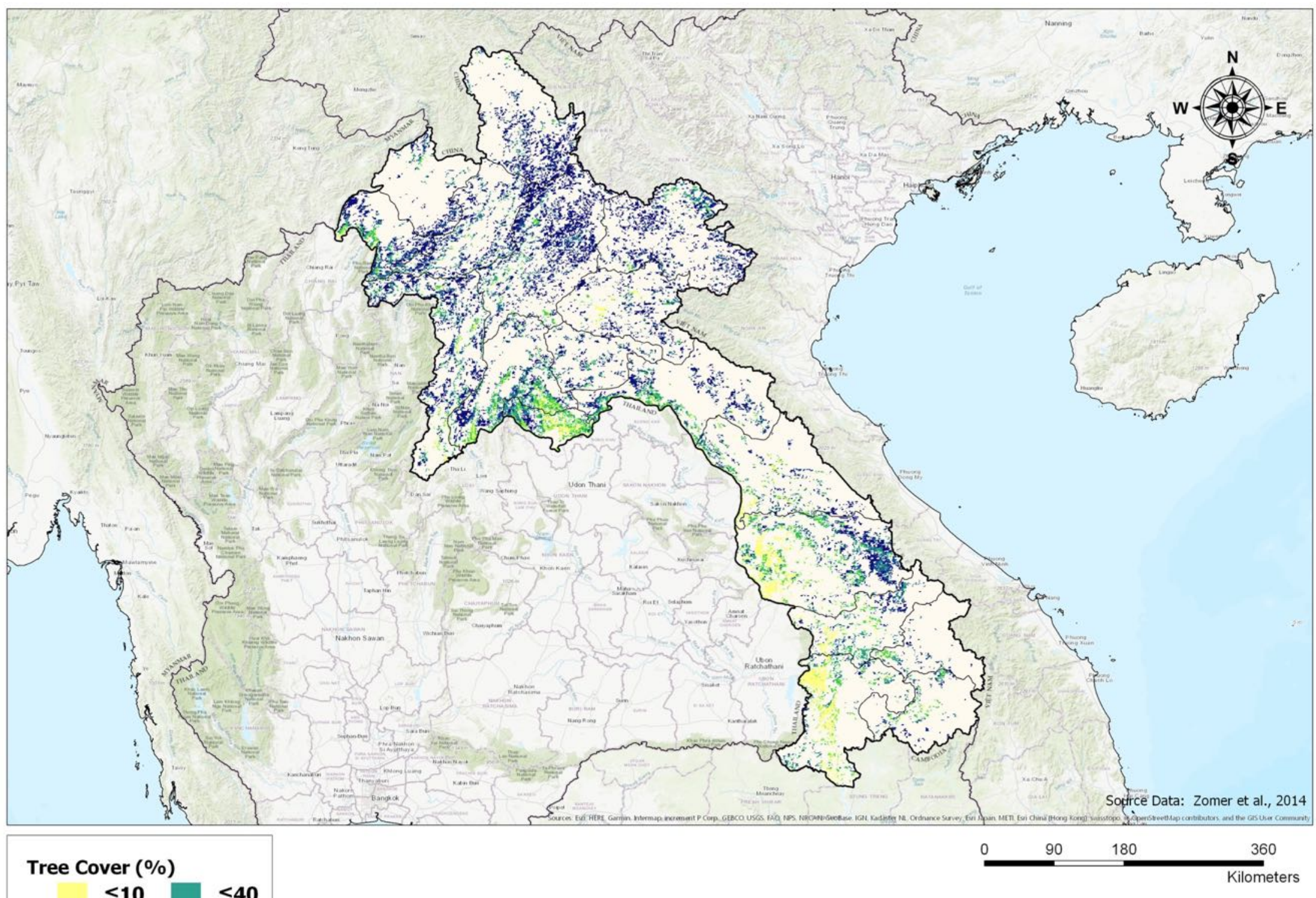

\section{Laos}


Asia Pacific Region : Change in Tree Cover on Agricultural Land (2000 - 2010)

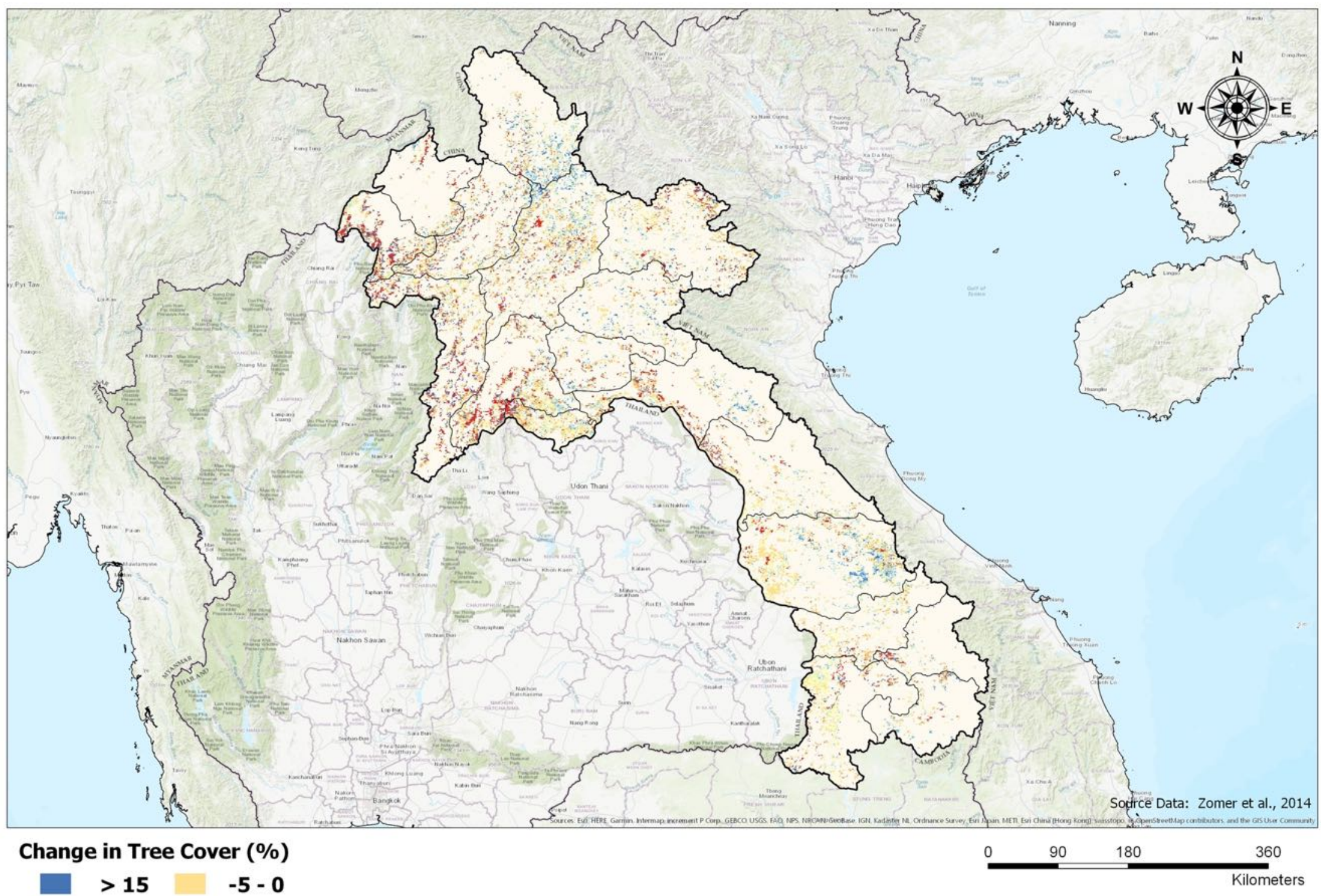
$>15 \quad-5-0$
$5-10 \quad-15--10$

\section{Laos}

$0-5 \square \leq-15$ 
Average Percent Tree Cover by Province, and Areal Extent of Tree Cover Classes $\left(\mathrm{km}^{2}\right)$

\begin{tabular}{|c|c|c|c|c|c|c|c|c|c|c|c|c|c|c|c|c|}
\hline \multicolumn{2}{|c|}{ Malaysia } & \multirow{2}{*}{\multicolumn{3}{|c|}{\begin{tabular}{|c|} 
Average Tree Cover by Province \\
Mean Tree Cover Percent by Province
\end{tabular}}} & \multicolumn{12}{|c|}{ Areal Extent of Tree Cover Class $\left(\mathrm{km}^{2}\right)$} \\
\hline \multirow{3}{*}{ Province } & \multirow{3}{*}{ Area_km } & & & & \multicolumn{4}{|c|}{ Year 2000} & \multirow{2}{*}{\multicolumn{4}{|c|}{ Year 2010}} & \multicolumn{4}{|c|}{ Change (2000 to 2010) } \\
\hline & & & $(\%)$ & & & a by Tree $\mathrm{C}$ & ver Class $(k$ & & & & & & & by Tree $\mathrm{C}$ & er Class $(k$ & \\
\hline & & 2000 & 2010 & Change & $<10 \%$ & $10-20 \%$ & $20-30 \%$ & $>30 \%$ & $<10 \%$ & $10-20 \%$ & $20-30 \%$ & $>30 \%$ & $<10 \%$ & $10-20 \%$ & $20-30 \%$ & $>\mathbf{3 0 \%}$ \\
\hline Johor & 11,552 & 44.13 & 43.83 & -0.31 & 99 & 885 & 1,568 & 9,000 & 250 & 1,155 & 1,592 & 8,555 & 151 & 270 & 24 & -445 \\
\hline Kedah & 4,839 & 39.13 & 39.26 & 0.13 & 42 & 505 & 1,163 & 3,129 & 65 & 804 & 793 & 3,177 & 23 & 299 & -370 & 48 \\
\hline Kelantan & 5,224 & 45.65 & 42.10 & -3.54 & 72 & 766 & 523 & 3,863 & 94 & 783 & 666 & 3,681 & 22 & 17 & 143 & -182 \\
\hline Melaka & 921 & 37.88 & 44.52 & 6.64 & 12 & 108 & 209 & 592 & 3 & 65 & 151 & 702 & -9 & -43 & -58 & 110 \\
\hline Negeri Sembilan & 4,024 & 40.84 & 44.06 & 3.22 & 85 & 402 & 682 & 2,855 & 117 & 290 & 554 & 3,063 & 32 & -112 & -128 & 208 \\
\hline Pahang & 13,764 & 49.50 & 52.12 & 2.62 & 98 & 872 & 1,389 & 11,405 & 80 & 600 & 1,176 & 11,908 & -18 & -272 & -213 & 503 \\
\hline Perak & 7,742 & 45.27 & 46.31 & 1.04 & 221 & 711 & 822 & 5,988 & 99 & 718 & 797 & 6,128 & -122 & 7 & -25 & 140 \\
\hline Perlis & 515 & 28.23 & 27.83 & -0.40 & 86 & 144 & 110 & 175 & 23 & 250 & 90 & 152 & -63 & 106 & -20 & -23 \\
\hline Pulau Pinang & 123 & 32.02 & 23.58 & -8.44 & 9 & 37 & 17 & 60 & 24 & 43 & 26 & 30 & 15 & 6 & 9 & -30 \\
\hline Putrajaya & 51 & 9.27 & 8.78 & -0.49 & 38 & 8 & 2 & 3 & 41 & 6 & 4 & 0 & 3 & -2 & 2 & -3 \\
\hline Sabah & 26,046 & 49.35 & 58.45 & 9.10 & 70 & 1,120 & 2,272 & 22,584 & 15 & 359 & 1,169 & 24,503 & -55 & -761 & $-1,103$ & 1,919 \\
\hline Sarawak & 25,664 & 49.62 & 57.98 & 8.36 & 145 & 1,144 & 2,115 & 22,260 & 95 & 514 & 1,119 & 23,936 & -50 & -630 & -996 & 1,676 \\
\hline Selangor & 2,980 & 37.62 & 35.32 & -2.29 & 255 & 473 & 459 & 1,793 & 212 & 613 & 509 & 1,646 & -43 & 140 & 50 & -147 \\
\hline Trengganu & 3,336 & 40.38 & 42.43 & 2.04 & 106 & 371 & 518 & 2,341 & 98 & 356 & 450 & 2,432 & -8 & -15 & -68 & 91 \\
\hline Malaysia - All & 106,780 & 46.76 & 51.37 & 4.61 & 1,338 & 7,546 & 11,849 & 86,048 & 1,216 & 6,556 & 9,096 & 89,913 & -122 & -990 & $-2,753$ & 3,865 \\
\hline
\end{tabular}

Average Percent Tree Cover by Province, and Areal Extent of Tree Cover Classes as a Percent of the Total Agricultural Area (\%)

\begin{tabular}{|c|c|c|c|c|c|c|c|c|c|c|c|c|c|c|c|c|}
\hline \multicolumn{2}{|c|}{ Malaysia } & \multirow{3}{*}{\multicolumn{3}{|c|}{$\begin{array}{c}\text { Average Tree Cover by Province } \\
\text { Percent Tree Cover } \\
(\%)\end{array}$}} & \multicolumn{12}{|c|}{ Areal Extent of Tree Cover Class as a Percent of Total Agricultural Area (\%) } \\
\hline \multirow{3}{*}{ Province } & \multirow{3}{*}{ Area_km } & & & & \multirow{2}{*}{\multicolumn{4}{|c|}{$\begin{array}{c}\text { Year } 2000 \\
\text { Percent of Total Agrciultural Area (\%) }\end{array}$}} & \multirow{2}{*}{\multicolumn{4}{|c|}{$\begin{array}{l}\text { Year } 2010 \\
\text { Percent of Total Agrciultural Area (\%) }\end{array}$}} & \multirow{2}{*}{\multicolumn{4}{|c|}{ Percent of Total Agrciultural Area (\%) }} \\
\hline & & & & & & & & & & & & & & & & \\
\hline & & 2000 & 2010 & Change & $<10 \%$ & $10-20 \%$ & $20-30 \%$ & $>30 \%$ & $<10 \%$ & $10-20 \%$ & $20-30 \%$ & $>30 \%$ & $<10 \%$ & $10-20 \%$ & $20-30 \%$ & $>30 \%$ \\
\hline Johor & 11,552 & 44.13 & 43.83 & -0.31 & 0.86 & 7.66 & 13.57 & 77.91 & 2.16 & 10.00 & 13.78 & 74.06 & 1.31 & 2.34 & 0.21 & -3.85 \\
\hline Kedah & 4,839 & 39.13 & 39.26 & 0.13 & 0.87 & 10.44 & 24.03 & 64.66 & 1.34 & 16.62 & 16.39 & 65.65 & 0.48 & 6.18 & -7.65 & 0.99 \\
\hline Kelantan & 5,224 & 45.65 & 42.10 & -3.54 & 1.38 & 14.66 & 10.01 & 73.95 & 1.80 & 14.99 & 12.75 & 70.46 & 0.42 & 0.33 & 2.74 & -3.48 \\
\hline Melaka & 921 & 37.88 & 44.52 & 6.64 & 1.30 & 11.73 & 22.69 & 64.28 & 0.33 & 7.06 & 16.40 & 76.22 & -0.98 & -4.67 & -6.30 & 11.94 \\
\hline Negeri Sembilan & 4,024 & 40.84 & 44.06 & 3.22 & 2.11 & 9.99 & 16.95 & 70.95 & 2.91 & 7.21 & 13.77 & 76.12 & 0.80 & -2.78 & -3.18 & 5.17 \\
\hline Pahang & 13,764 & 49.50 & 52.12 & 2.62 & 0.71 & 6.34 & 10.09 & 82.86 & 0.58 & 4.36 & 8.54 & 86.52 & -0.13 & -1.98 & -1.55 & 3.65 \\
\hline Perak & 7,742 & 45.27 & 46.31 & 1.04 & 2.85 & 9.18 & 10.62 & 77.34 & 1.28 & 9.27 & 10.29 & 79.15 & -1.58 & 0.09 & -0.32 & 1.81 \\
\hline Perlis & 515 & 28.23 & 27.83 & -0.40 & 16.70 & 27.96 & 21.36 & 33.98 & 4.47 & 48.54 & 17.48 & 29.51 & -12.23 & 20.58 & -3.88 & -4.47 \\
\hline Pulau Pinang & 123 & 32.02 & 23.58 & -8.44 & 7.32 & 30.08 & 13.82 & 48.78 & 19.51 & 34.96 & 21.14 & 24.39 & 12.20 & 4.88 & 7.32 & -24.39 \\
\hline Putrajaya & 51 & 9.27 & 8.78 & $\begin{array}{l}-0.49 \\
-0.44\end{array}$ & 74.51 & 15.69 & 3.92 & 5.88 & 80.39 & 11.76 & 7.84 & 0.00 & 5.88 & $\begin{array}{l}-3.92 \\
\end{array}$ & 3.92 & -5.88 \\
\hline Sabah & 26,046 & 49.35 & 58.45 & 9.10 & 0.27 & 4.30 & 8.72 & 86.71 & 0.06 & 1.38 & 4.49 & 94.08 & -0.21 & -2.92 & -4.23 & 7.37 \\
\hline Sarawak & 25,664 & 49.62 & 57.98 & 8.36 & 0.56 & 4.46 & 8.24 & 86.74 & 0.37 & 2.00 & 4.36 & 93.27 & -0.19 & -2.45 & -3.88 & 6.53 \\
\hline Selangor & 2,980 & 37.62 & 35.32 & -2.29 & 8.56 & 15.87 & 15.40 & 60.17 & 7.11 & 20.57 & 17.08 & 55.24 & -1.44 & 4.70 & 1.68 & -4.93 \\
\hline Trengganu & 3,336 & 40.38 & 42.43 & 2.04 & 3.18 & 11.12 & 15.53 & 70.17 & 2.94 & 10.67 & 13.49 & 72.90 & -0.24 & -0.45 & -2.04 & 2.73 \\
\hline Malaysia - All & 106,780 & 46.76 & 51.37 & 4.61 & 1.25 & 7.07 & 11.10 & 80.58 & 1.14 & 6.14 & 8.52 & 84.20 & -0.11 & -0.93 & -2.58 & 3.62 \\
\hline
\end{tabular}


Asia Pacific Region : Tree Cover on Agricultural Land (2010)

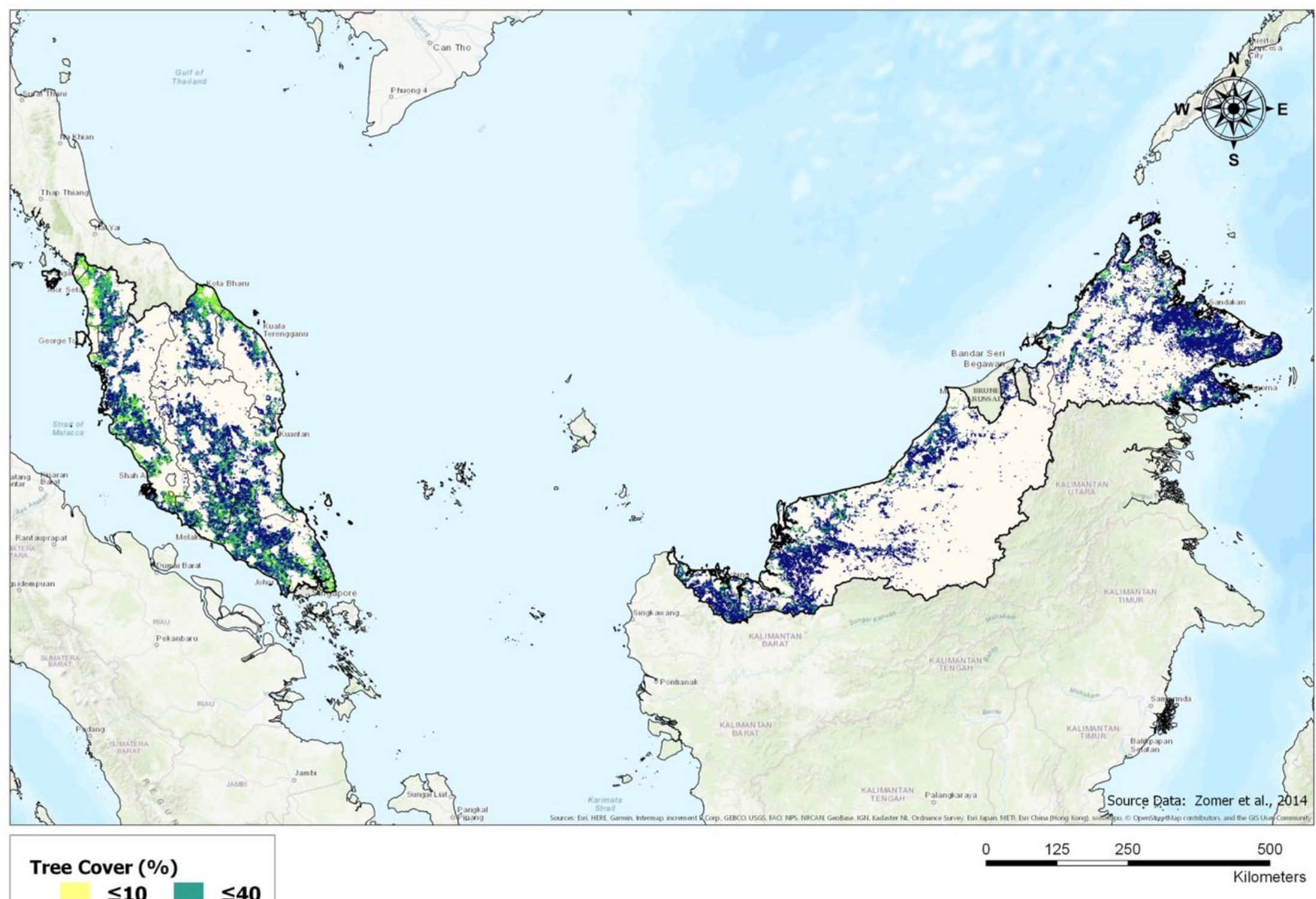

Malaysia 
Asia Pacific Region : Change in Tree Cover on Agricultural Land (2000 - 2010)

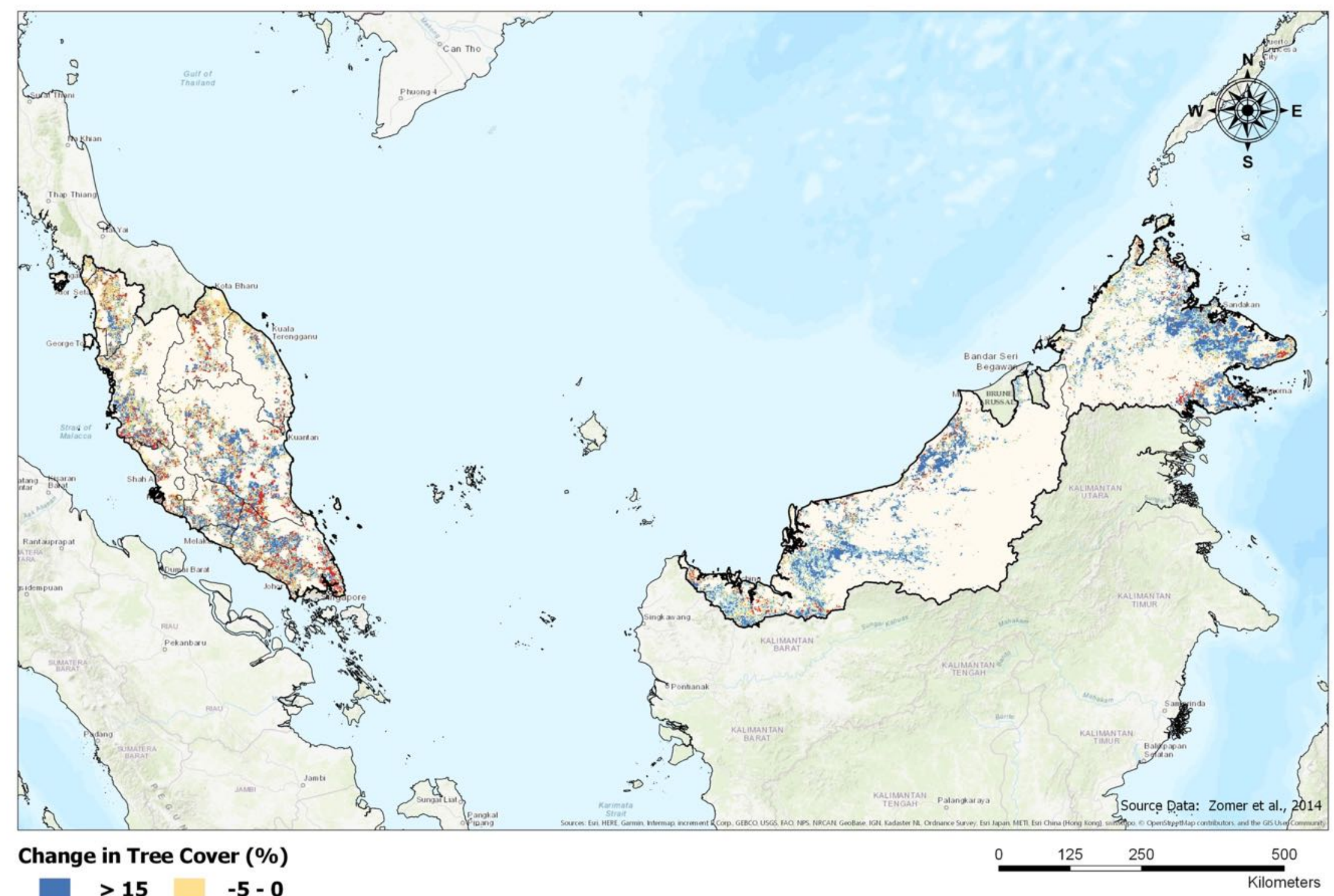

\begin{tabular}{|r|r|}
\hline$>15$ & $-5-0$ \\
$10-15$ & $-10--5$ \\
$5-10$ & $-15--10$ \\
$0-5$ & $\leq-15$
\end{tabular}

Malaysia 
Appendix 13: Mongolia

Average Percent Tree Cover by Province, and Areal Extent of Tree Cover Classes $\left(\mathrm{km}^{2}\right)$

\begin{tabular}{|c|c|c|c|c|c|c|c|c|c|c|c|c|c|c|c|c|}
\hline \multicolumn{2}{|c|}{ Mongolia } & \multirow{3}{*}{\multicolumn{3}{|c|}{\begin{tabular}{|c|} 
Average Tree Cover by Province \\
Mean Tree Cover Percent by Province \\
$(\%)$
\end{tabular}}} & \multicolumn{12}{|c|}{ Areal Extent of Tree Cover Class $\left(\mathrm{km}^{2}\right)$} \\
\hline \multirow{3}{*}{ Province } & \multirow{3}{*}{ Area_km } & & & & \multicolumn{4}{|c|}{ Year 2000} & \multicolumn{4}{|c|}{ Year 2010} & \multicolumn{4}{|c|}{ Change (2000 to 2010) } \\
\hline & & & & & & ea by Tree & ver Class & & & rea by Tree & ver Class & & & by Tree $\mathrm{C}$ & Jer Class (k & \\
\hline & & 2000 & 2010 & Change & $<10 \%$ & $10-20 \%$ & $20-30 \%$ & $>30 \%$ & $<10 \%$ & $10-20 \%$ & $20-30 \%$ & $>30 \%$ & $<10 \%$ & $10-20 \%$ & $20-30 \%$ & $>\mathbf{3 0} \%$ \\
\hline Arhangay & 26,770 & 4.49 & 4.38 & -0.12 & 24,466 & 1,991 & 274 & 39 & 24,033 & 2,085 & 502 & 150 & -433 & 94 & 228 & 111 \\
\hline Bayan-Ölgiy & 3,922 & 2.44 & 2.32 & -0.12 & 3,886 & 35 & 1 & 0 & 3,881 & 41 & 0 & 0 & -5 & 6 & -1 & 0 \\
\hline Bayanhongor & 10,085 & 2.40 & 2.02 & -0.38 & 10,044 & 39 & 2 & 0 & 10,040 & 45 & 0 & 0 & -4 & 6 & -2 & 0 \\
\hline Bulgan & 16,841 & 5.07 & 5.57 & 0.49 & 15,533 & 1,217 & 86 & 5 & 14,714 & 1,651 & 416 & 60 & -819 & 434 & 330 & 55 \\
\hline Darhan-Uul & 1,227 & 6.07 & 5.91 & -0.16 & 1,115 & 91 & 16 & 5 & 1,101 & 96 & 17 & 13 & -14 & 5 & 1 & 8 \\
\hline Dornod & 58,423 & 3.89 & 3.37 & -0.52 & 57,939 & 458 & 23 & 3 & 58,062 & 346 & 14 & 1 & 123 & -112 & -9 & -2 \\
\hline Dornogovi & 205 & 1.09 & 1.06 & -0.03 & 205 & 0 & 0 & 0 & 205 & 0 & 0 & 0 & 0 & 0 & 0 & 0 \\
\hline Dundgovi & 10 & 0.90 & 1.50 & 0.60 & 10 & 0 & 0 & 0 & 10 & 0 & 0 & 0 & 0 & 0 & 0 & 0 \\
\hline Dzavhan & 3,173 & 5.78 & 6.01 & 0.23 & 2,809 & 344 & 20 & 0 & 2,692 & 418 & 56 & 7 & -117 & 74 & 36 & 7 \\
\hline Govi-Altay & 2,621 & 1.42 & 1.69 & 0.27 & 2,609 & 12 & 0 & 0 & 2,617 & 4 & 0 & 0 & 8 & -8 & 0 & 0 \\
\hline Govisümber & 135 & 1.69 & 0.05 & -1.64 & 135 & 0 & 0 & 0 & 135 & 0 & 0 & 0 & 0 & 0 & 0 & 0 \\
\hline Hentiy & 33,308 & 4.57 & 4.21 & -0.37 & 30,981 & 2,070 & 242 & 15 & 30,347 & 2,438 & 429 & 94 & -634 & 368 & 187 & 79 \\
\hline Hovd & 5,330 & 1.69 & 1.89 & 0.19 & 5,242 & 83 & 5 & 0 & 5,261 & 60 & 9 & 0 & 19 & -23 & 4 & 0 \\
\hline Hövsgöl & 26,259 & 6.02 & 7.22 & 1.21 & 22,671 & 3,084 & 477 & 27 & 20,382 & 4,135 & 1,407 & 335 & $-2,289$ & 1,051 & 930 & 308 \\
\hline Ömnögovi & 267 & 1.12 & 1.59 & 0.47 & 267 & 0 & 0 & 0 & 267 & 0 & 0 & 0 & 0 & 0 & 0 & 0 \\
\hline Orhon & 291 & 4.42 & 4.14 & -0.27 & 278 & 10 & 3 & 0 & 276 & 12 & 3 & 0 & -2 & 2 & 0 & 0 \\
\hline Övörhangay & 6,980 & 3.34 & 2.90 & -0.45 & 6,599 & 314 & 52 & 15 & 6,611 & 263 & 79 & 27 & 12 & -51 & 27 & 12 \\
\hline Selenge & 12,164 & 7.19 & 6.73 & -0.46 & 10,315 & 1,538 & 226 & 85 & 10,268 & 1,452 & 309 & 135 & -47 & -86 & 83 & 50 \\
\hline Sühbaatar & 19,054 & 3.02 & 2.74 & -0.27 & 19,050 & 4 & 0 & 0 & 19,051 & 3 & 0 & 0 & 1 & -1 & 0 & 0 \\
\hline Töv & 11,638 & 4.99 & 4.89 & -0.11 & 10,590 & 839 & 170 & 39 & 10,261 & 936 & 309 & 132 & -329 & 97 & 139 & 93 \\
\hline Ulaanbaatar & 1,181 & 5.84 & 4.23 & -1.61 & 1,044 & 96 & 36 & 5 & 1,067 & 83 & 23 & 8 & 23 & -13 & -13 & 3 \\
\hline Uvs & 4,672 & 3.85 & 4.44 & 0.60 & 4,466 & 196 & 10 & 0 & 4,273 & 343 & 49 & 7 & -193 & 147 & 39 & 7 \\
\hline Mongolia - All & 244.556 & 4.37 & 4.28 & -0.09 & 230,256 & 12.421 & 1,643 & 238 & 225,556 & 14.411 & 3,622 & 969 & $-4,700$ & 1,990 & 1,979 & 731 \\
\hline
\end{tabular}


Average Percent Tree Cover by Province, and Areal Extent of Tree Cover Classes as a Percent of the Total Agricultural Area (\%)

\begin{tabular}{|c|c|c|c|c|c|c|c|c|c|c|c|c|c|c|c|c|}
\hline \multicolumn{2}{|c|}{ Mongolia } & \multirow{3}{*}{\multicolumn{3}{|c|}{$\begin{array}{c}\text { Average Tree Cover by Province } \\
\text { Percent Tree Cover } \\
(\%)\end{array}$}} & \multicolumn{12}{|c|}{ Areal Extent of Tree Cover Class as a Percent of Total Agricultural Area (\%) } \\
\hline \multirow{3}{*}{ Province } & \multirow{3}{*}{ Area_km } & & & & \multirow{2}{*}{\multicolumn{4}{|c|}{$\begin{array}{c}\text { Year } 2000 \\
\text { Percent of Total Agrciultural Area (\%) }\end{array}$}} & & Yeal & & & & Change (2 & 0 to 2010 & \\
\hline & & & & & & ti of Total A & & & \multicolumn{4}{|c|}{ Percent of Total Agrciultural Area ( \%) } & \multicolumn{4}{|c|}{ Percent of Total Agrciultural Area (\%) } \\
\hline & & 2000 & 2010 & Change & $<10 \%$ & $10-20 \%$ & $20-30 \%$ & $>30 \%$ & $<10 \%$ & $10-20 \%$ & $20-30 \%$ & $>30 \%$ & $<10 \%$ & $10-20 \%$ & $20-30 \%$ & $>30 \%$ \\
\hline Arhangay & 26,770 & 4.49 & 4.38 & -0.12 & 91.39 & 7.44 & 1.02 & 0.15 & 89.78 & 7.79 & 1.88 & 0.56 & -1.62 & 0.35 & 0.85 & 0.41 \\
\hline Bayan-Ölgiy & 3,922 & 2.44 & 2.32 & -0.12 & 99.08 & 0.89 & 0.03 & 0.00 & 98.96 & 1.05 & 0.00 & 0.00 & -0.13 & 0.15 & -0.03 & 0.00 \\
\hline Bayanhongor & 10,085 & 2.40 & 2.02 & -0.38 & 99.59 & 0.39 & 0.02 & 0.00 & 99.55 & 0.45 & 0.00 & 0.00 & -0.04 & 0.06 & -0.02 & 0.00 \\
\hline Bulgan & 16,841 & 5.07 & 5.57 & 0.49 & 92.23 & 7.23 & 0.51 & 0.03 & 87.37 & 9.80 & 2.47 & 0.36 & -4.86 & 2.58 & 1.96 & 0.33 \\
\hline Darhan-Uul & 1,227 & 6.07 & 5.91 & -0.16 & 90.87 & 7.42 & 1.30 & 0.41 & 89.73 & 7.82 & 1.39 & 1.06 & -1.14 & 0.41 & 0.08 & 0.65 \\
\hline Dornod & 58,423 & 3.89 & 3.37 & -0.52 & 99.17 & 0.78 & 0.04 & 0.01 & 99.38 & 0.59 & 0.02 & 0.00 & 0.21 & -0.19 & -0.02 & 0.00 \\
\hline Dornogovi & 205 & 1.09 & 1.06 & -0.03 & 100.00 & 0.00 & 0.00 & 0.00 & 100.00 & 0.00 & 0.00 & 0.00 & 0.00 & 0.00 & 0.00 & 0.00 \\
\hline Dundgovi & 10 & 0.90 & 1.50 & 0.60 & 100.00 & 0.00 & 0.00 & 0.00 & 100.00 & 0.00 & 0.00 & 0.00 & 0.00 & 0.00 & 0.00 & 0.00 \\
\hline Dzavhan & 3,173 & 5.78 & 6.01 & 0.23 & 88.53 & 10.84 & 0.63 & 0.00 & 84.84 & 13.17 & 1.76 & 0.22 & -3.69 & 2.33 & 1.13 & 0.22 \\
\hline Govi-Altay & 2,621 & 1.42 & 1.69 & 0.27 & 99.54 & 0.46 & 0.00 & 0.00 & 99.85 & 0.15 & 0.00 & 0.00 & 0.31 & -0.31 & 0.00 & 0.00 \\
\hline Govisümber & 135 & 1.69 & 0.05 & -1.64 & 100.00 & 0.00 & 0.00 & 0.00 & 100.00 & 0.00 & 0.00 & 0.00 & 0.00 & 0.00 & 0.00 & 0.00 \\
\hline Hentiy & 33,308 & 4.57 & 4.21 & -0.37 & 93.01 & 6.21 & 0.73 & 0.05 & 91.11 & 7.32 & 1.29 & 0.28 & -1.90 & 1.10 & 0.56 & 0.24 \\
\hline Hovd & 5,330 & 1.69 & 1.89 & 0.19 & 98.35 & 1.56 & 0.09 & 0.00 & 98.71 & 1.13 & 0.17 & 0.00 & 0.36 & -0.43 & 0.08 & 0.00 \\
\hline Hövsgöl & 26,259 & 6.02 & 7.22 & 1.21 & 86.34 & 11.74 & 1.82 & 0.10 & 77.62 & 15.75 & 5.36 & 1.28 & -8.72 & 4.00 & 3.54 & 1.17 \\
\hline Ömnögovi & 267 & 1.12 & 1.59 & 0.47 & 100.00 & 0.00 & 0.00 & 0.00 & 100.00 & 0.00 & 0.00 & 0.00 & 0.00 & 0.00 & 0.00 & 0.00 \\
\hline Orhon & 291 & 4.42 & 4.14 & -0.27 & 95.53 & 3.44 & 1.03 & 0.00 & 94.85 & 4.12 & 1.03 & 0.00 & -0.69 & 0.69 & 0.00 & 0.00 \\
\hline Övörhangay & 6,980 & 3.34 & 2.90 & -0.45 & 94.54 & 4.50 & 0.74 & 0.21 & 94.71 & 3.77 & 1.13 & 0.39 & 0.17 & -0.73 & 0.39 & 0.17 \\
\hline Selenge & 12,164 & 7.19 & 6.73 & -0.46 & 84.80 & 12.64 & 1.86 & 0.70 & 84.41 & 11.94 & 2.54 & 1.11 & -0.39 & -0.71 & 0.68 & 0.41 \\
\hline Sühbaatar & 19,054 & 3.02 & 2.74 & -0.27 & 99.98 & 0.02 & 0.00 & 0.00 & 99.99 & 0.02 & 0.00 & 0.00 & 0.01 & -0.01 & 0.00 & 0.00 \\
\hline Töv & 11,638 & 4.99 & 4.89 & -0.11 & 91.00 & 7.21 & 1.46 & 0.34 & 88.17 & 8.04 & 2.66 & 1.13 & -2.83 & 0.83 & 1.19 & 0.80 \\
\hline Ulaanbaatar & 1,181 & 5.84 & 4.23 & -1.61 & 88.40 & 8.13 & 3.05 & 0.42 & 90.35 & 7.03 & 1.95 & 0.68 & 1.95 & -1.10 & -1.10 & 0.25 \\
\hline Uvs & 4,672 & 3.85 & 4.44 & 0.60 & 95.59 & 4.20 & 0.21 & 0.00 & 91.46 & 7.34 & 1.05 & 0.15 & -4.13 & 3.15 & 0.83 & 0.15 \\
\hline Mongolia - All & 244,556 & 4.37 & 4.28 & -0.09 & 94.15 & 5.08 & 0.67 & 0.10 & 92.23 & 5.89 & 1.48 & 0.40 & -1.92 & 0.81 & 0.81 & 0.30 \\
\hline
\end{tabular}


Asia Pacific Region : Tree Cover on Agricultural Land (2010)

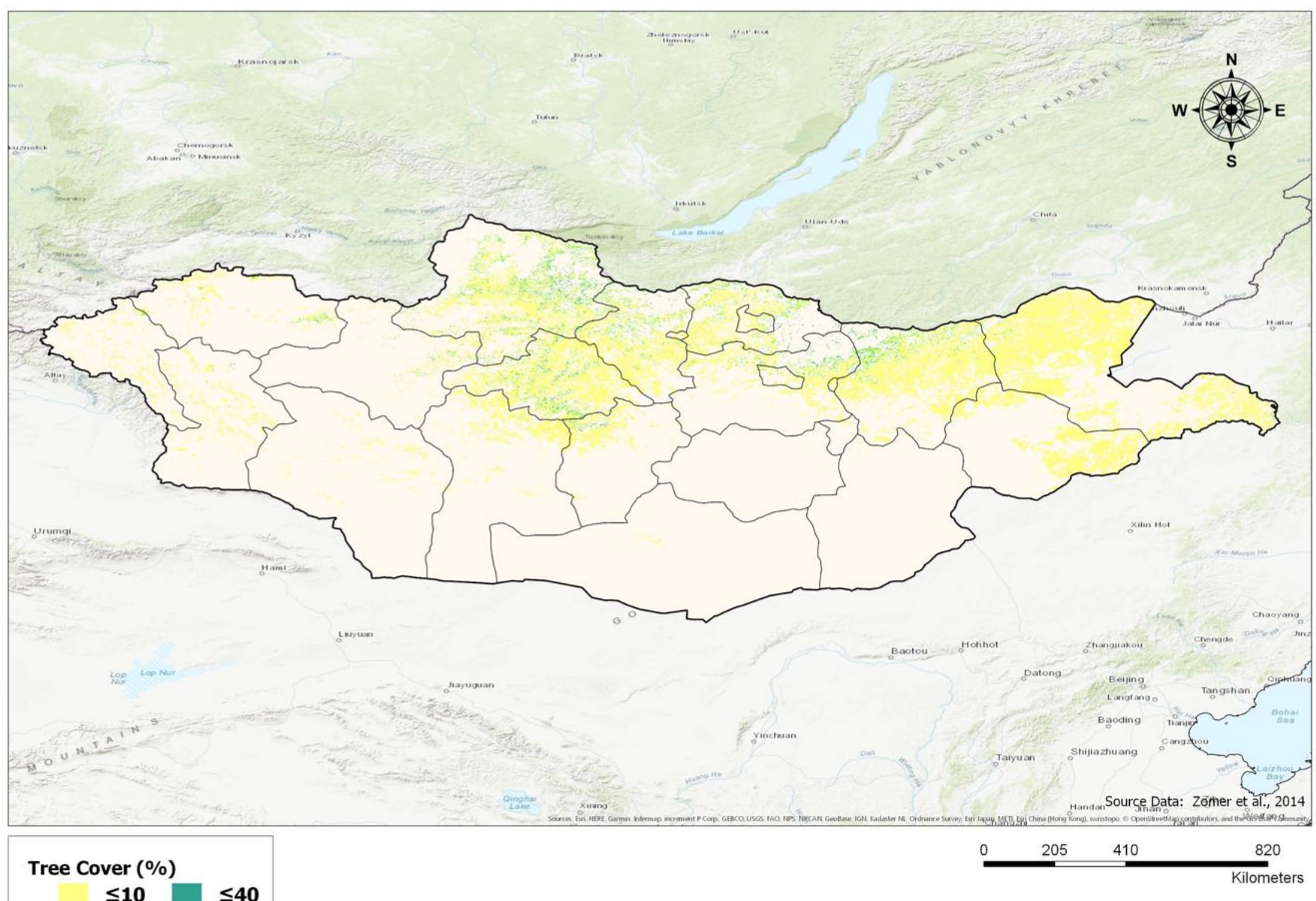

Mongolia 
Asia Pacific Region : Change in Tree Cover on Agricultural Land (2000 - 2010)

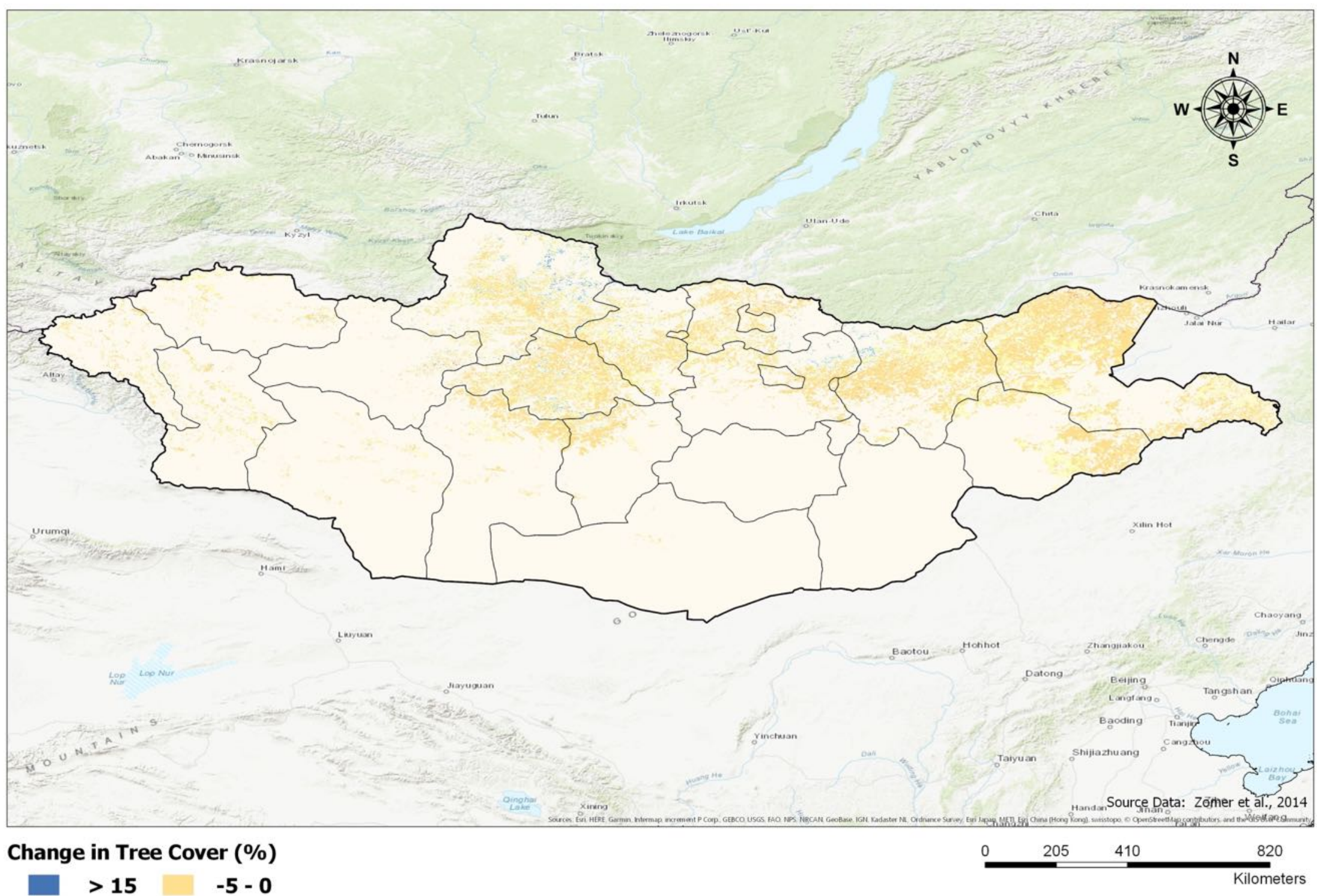

Mongolia 
Average Percent Tree Cover by Province, and Areal Extent of Tree Cover Classes $\left(\mathrm{km}^{2}\right)$

\begin{tabular}{|c|c|c|c|c|c|c|c|c|c|c|c|c|c|c|c|c|}
\hline \multicolumn{2}{|c|}{ Myanmar } & \multirow{2}{*}{\multicolumn{3}{|c|}{\begin{tabular}{|c|} 
Average Tree Cover by Province \\
Mean Tree Cover Percent by Province
\end{tabular}}} & \multicolumn{12}{|c|}{ Areal Extent of Tree Cover Class $\left(\mathrm{km}^{2}\right)$} \\
\hline \multirow{3}{*}{ Province } & \multirow{3}{*}{ Area_km } & & & & \multicolumn{4}{|c|}{ Year 2000} & \multicolumn{4}{|c|}{ Year 2010} & \multicolumn{4}{|c|}{ Change (2000 to 2010) } \\
\hline & & & $(\%)$ & & & ea by Tree & ver Class (I & & & ea by Tree & er Class & & & a by Tree $C$ & ver Class & \\
\hline & & 2000 & 2010 & Change & $<10 \%$ & $10-20 \%$ & $20-30 \%$ & $>30 \%$ & $<10 \%$ & $10-20 \%$ & $20-30 \%$ & $>30 \%$ & $<10 \%$ & $10-20 \%$ & $20-30 \%$ & $>\mathbf{3 0 \%}$ \\
\hline Ayeyarwady & 21,228 & 9.52 & 10.06 & 0.54 & 15,716 & 3,223 & 1,080 & 1,209 & 13,873 & 5,716 & 1,151 & 488 & $-1,843$ & 2,493 & 71 & -721 \\
\hline Bago & 16,906 & 11.59 & 10.24 & -1.35 & 12,705 & 1,425 & 615 & 2,161 & 12,951 & 1,773 & 622 & 1,560 & 246 & 348 & 7 & -601 \\
\hline Chin & 4,413 & 53.53 & 38.90 & -14.63 & 72 & 69 & 106 & 4,166 & 81 & 89 & 477 & 3,766 & 9 & 20 & 371 & -400 \\
\hline Kachin & 9,189 & 49.71 & 48.86 & -0.86 & 1,081 & 576 & 561 & 6,971 & 1,038 & 672 & 660 & 6,819 & -43 & 96 & 99 & -152 \\
\hline Kayah & 1,333 & 30.31 & 26.04 & -4.27 & 415 & 77 & 181 & 660 & 446 & 209 & 149 & 529 & 31 & 132 & -32 & -131 \\
\hline Kayin & 8,250 & 36.23 & 33.56 & -2.67 & 1,892 & 757 & 651 & 4,950 & 1,671 & 1,213 & 877 & 4,489 & -221 & 456 & 226 & -461 \\
\hline Magway & 27,480 & 6.00 & 4.44 & -1.56 & 22,965 & 2,469 & 1,355 & 691 & 24,513 & 2,082 & 568 & 317 & 1,548 & -387 & -787 & -374 \\
\hline Mandalay & 22,833 & 8.36 & 6.97 & -1.39 & 18,725 & 1,342 & 859 & 1,907 & 19,275 & 1,678 & 468 & 1,412 & 550 & 336 & -391 & -495 \\
\hline Mon & 5,040 & 22.17 & 21.14 & -1.03 & 2,435 & 326 & 330 & 1,949 & 2,276 & 504 & 491 & 1,769 & -159 & 178 & 161 & -180 \\
\hline Naypyitaw & 126 & 13.50 & 3.02 & -10.48 & 58 & 39 & 18 & 11 & 125 & 1 & 0 & 0 & 67 & -38 & -18 & -11 \\
\hline Rakhine & 11,324 & 37.16 & 25.10 & -12.06 & 3,371 & 905 & 511 & 6,537 & 3,273 & 1,354 & 1,862 & 4,835 & -98 & 449 & 1,351 & $-1,702$ \\
\hline Sagaing & 29,873 & 12.16 & 11.44 & -0.71 & 21,275 & 2,678 & 2,031 & 3,889 & 21,690 & 3,737 & 1,337 & 3,109 & 415 & 1,059 & -694 & -780 \\
\hline Shan & 25,192 & 39.06 & 36.31 & -2.75 & 4,372 & 1,596 & 1,927 & 17,297 & 4,996 & 1,741 & 2,695 & 15,760 & 624 & 145 & 768 & $-1,537$ \\
\hline Tanintharyi & 3,448 & 37.21 & 35.28 & -1.93 & 338 & 296 & 450 & 2,364 & 262 & 354 & 667 & 2,165 & -76 & 58 & 217 & -199 \\
\hline Yangon & 6,328 & 5.73 & 6.40 & 0.68 & 5,649 & 326 & 92 & 261 & 5,598 & 447 & 68 & 215 & -51 & 121 & -24 & -46 \\
\hline Myanmar - All & 192,963 & 19.86 & 17.67 & -2.19 & 111,070 & 16,104 & 10,767 & 55,023 & 112,069 & 21,570 & 12,092 & 47,233 & 999 & 5,466 & 1,325 & $-7,790$ \\
\hline
\end{tabular}

Average Percent Tree Cover by Province, and Areal Extent of Tree Cover Classes as a Percent of the Total Agricultural Area (\%)

\begin{tabular}{|c|c|c|c|c|c|c|c|c|c|c|c|c|c|c|c|c|}
\hline \multicolumn{2}{|c|}{ Myanmar } & \multirow{3}{*}{\multicolumn{3}{|c|}{$\begin{array}{c}\text { Average Tree Cover by Province } \\
\text { Percent Tree Cover } \\
(\%)\end{array}$}} & \multicolumn{12}{|c|}{ Areal Extent of Tree Cover Class as a Percent of Total Agricultural Area (\%) } \\
\hline \multirow{3}{*}{ Province } & \multirow{3}{*}{ Area_km } & & & & \multirow{2}{*}{\multicolumn{4}{|c|}{$\begin{array}{c}\text { Year } 2000 \\
\text { Percent of Total Agrciultural Area (\%) }\end{array}$}} & & Year & 2010 & & & Change (2 & 00 to 2010) & \\
\hline & & & & & & & & & \multicolumn{4}{|c|}{ Percent of Total Agrciultural Area (\%) } & \multicolumn{4}{|c|}{ Percent of Total Agrciultural Area (\%) } \\
\hline & & 2000 & 2010 & Change & $<10 \%$ & $10-20 \%$ & $20-30 \%$ & $>30 \%$ & $<10 \%$ & $10-20 \%$ & $20-30 \%$ & $>30 \%$ & $<10 \%$ & $10-20 \%$ & $20-30 \%$ & $>30 \%$ \\
\hline Ayeyarwady & 21,228 & 9.52 & 10.06 & 0.54 & 74.03 & 15.18 & 5.09 & 5.70 & 65.35 & 26.93 & 5.42 & 2.30 & -8.68 & 11.74 & 0.33 & -3.40 \\
\hline Bago & 16,906 & 11.59 & 10.24 & -1.35 & 75.15 & 8.43 & 3.64 & 12.78 & 76.61 & 10.49 & 3.68 & 9.23 & 1.46 & 2.06 & 0.04 & -3.55 \\
\hline Chin & 4,413 & 53.53 & 38.90 & -14.63 & 1.63 & 1.56 & 2.40 & 94.40 & 1.84 & 2.02 & 10.81 & 85.34 & 0.20 & 0.45 & 8.41 & -9.06 \\
\hline Kachin & 9,189 & 49.71 & 48.86 & -0.86 & 11.76 & 6.27 & 6.11 & 75.86 & 11.30 & 7.31 & 7.18 & 74.21 & -0.47 & 1.04 & 1.08 & -1.65 \\
\hline Kayah & 1,333 & 30.31 & 26.04 & -4.27 & 31.13 & 5.78 & 13.58 & 49.51 & 33.46 & 15.68 & 11.18 & 39.69 & 2.33 & 9.90 & -2.40 & -9.83 \\
\hline Kayin & 8,250 & 36.23 & 33.56 & -2.67 & 22.93 & 9.18 & 7.89 & 60.00 & 20.25 & 14.70 & 10.63 & 54.41 & -2.68 & 5.53 & 2.74 & -5.59 \\
\hline Magway & 27,480 & 6.00 & 4.44 & -1.56 & 83.57 & 8.98 & 4.93 & 2.51 & 89.20 & 7.58 & 2.07 & 1.15 & 5.63 & -1.41 & -2.86 & -1.36 \\
\hline Mandalay & 22,833 & 8.36 & 6.97 & -1.39 & 82.01 & 5.88 & 3.76 & 8.35 & 84.42 & 7.35 & 2.05 & 6.18 & 2.41 & 1.47 & -1.71 & -2.17 \\
\hline Mon & 5,040 & 22.17 & 21.14 & -1.03 & 48.31 & 6.47 & 6.55 & 38.67 & 45.16 & 10.00 & 9.74 & 35.10 & -3.15 & 3.53 & 3.19 & -3.57 \\
\hline Naypyitaw & 126 & 13.50 & 3.02 & -10.48 & 46.03 & 30.95 & 14.29 & 8.73 & 99.21 & 0.79 & 0.00 & 0.00 & 53.18 & -30.16 & -14.29 & -8.73 \\
\hline Rakhine & 11,324 & 37.16 & 25.10 & -12.06 & 29.77 & 7.99 & 4.51 & 57.73 & 28.90 & 11.96 & 16.44 & 42.70 & -0.87 & 3.97 & 11.93 & -15.03 \\
\hline Sagaing & 29,873 & 12.16 & 11.44 & -0.71 & 71.22 & 8.96 & 6.80 & 13.02 & 72.61 & 12.51 & 4.48 & 10.41 & 1.39 & 3.55 & -2.32 & -2.61 \\
\hline Shan & 25,192 & 39.06 & 36.31 & -2.75 & 17.35 & 6.34 & 7.65 & 68.66 & 19.83 & 6.91 & 10.70 & 62.56 & 2.48 & 0.58 & 3.05 & -6.10 \\
\hline Tanintharyi & 3,448 & 37.21 & 35.28 & -1.93 & 9.80 & 8.58 & 13.05 & 68.56 & 7.60 & 10.27 & 19.34 & 62.79 & -2.20 & 1.68 & 6.29 & -5.77 \\
\hline Yangon & 6,328 & 5.73 & 6.40 & 0.68 & 89.27 & 5.15 & 1.45 & 4.12 & 88.46 & 7.06 & 1.07 & 3.40 & -0.81 & 1.91 & -0.38 & -0.73 \\
\hline Myanmar - All & 192,963 & 19.86 & 17.67 & -2.19 & 57.56 & 8.35 & 5.58 & 28.52 & 58.08 & 11.18 & 6.27 & 24.48 & 0.52 & 2.83 & 0.69 & -4.04 \\
\hline
\end{tabular}


Asia Pacific Region : Tree Cover on Agricultural Land (2010)

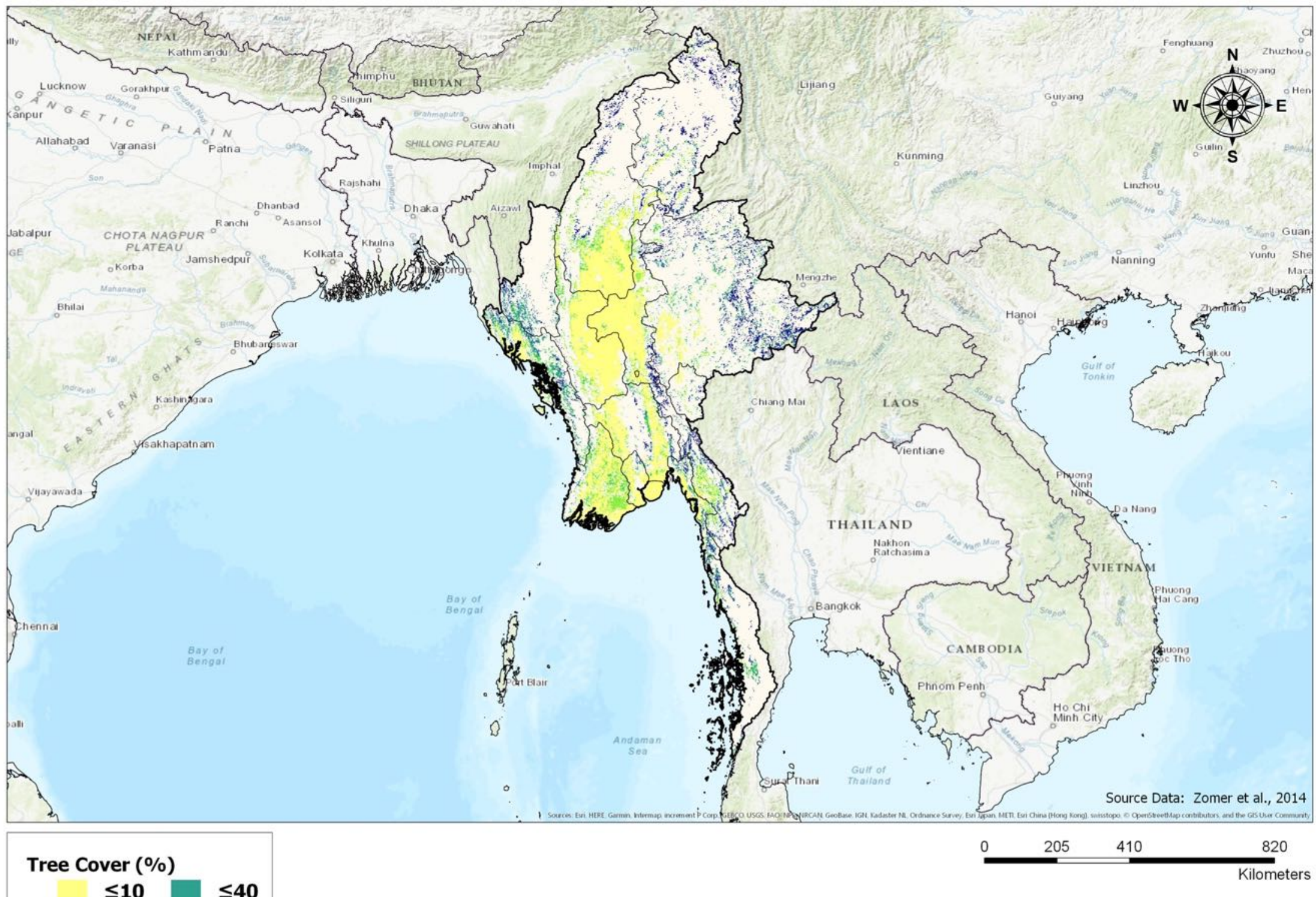

Myanmar 
Asia Pacific Region : Change in Tree Cover on Agricultural Land (2000 - 2010)

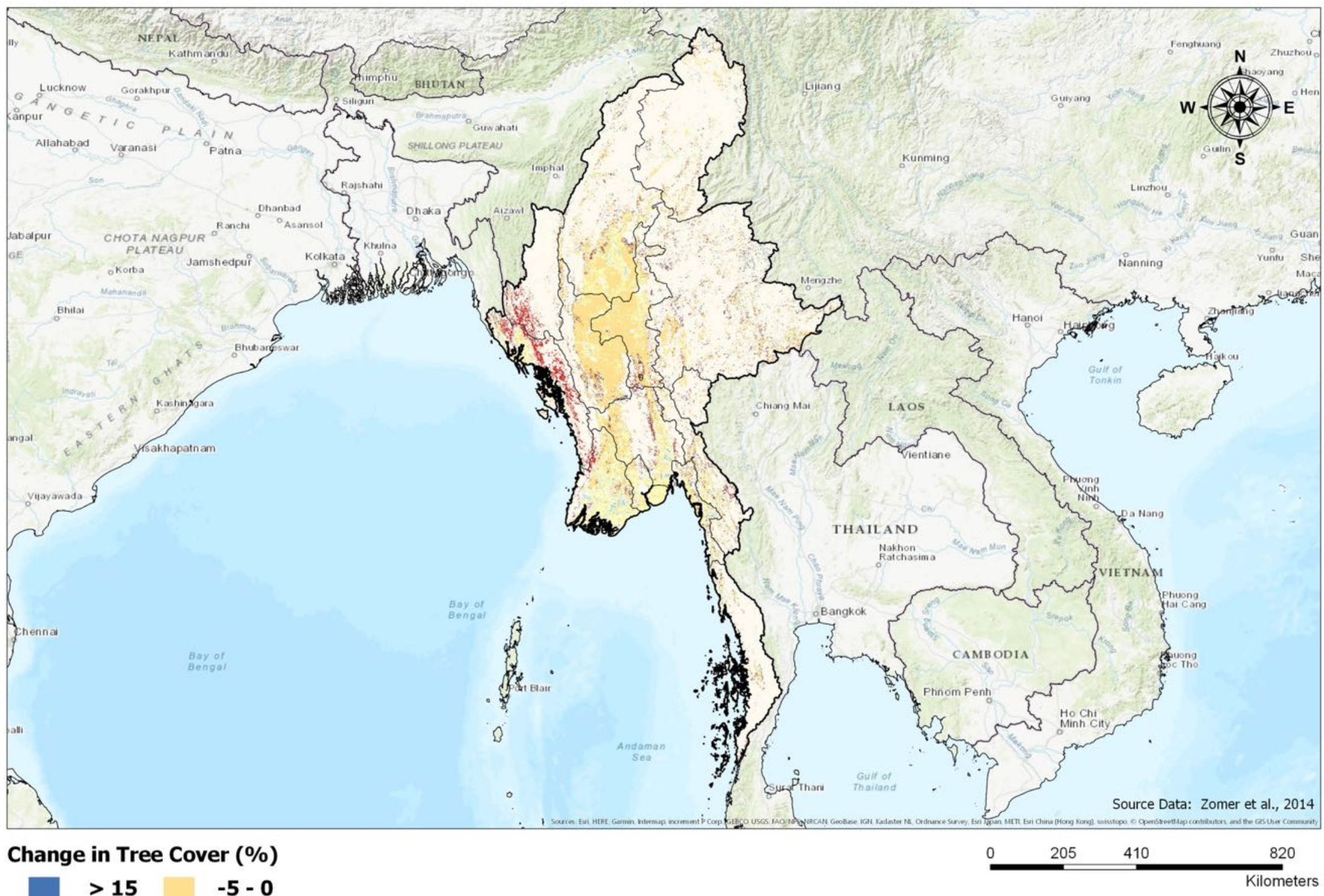

\begin{tabular}{|r|r|}
$>15$ & $-5-0$ \\
$10-15$ & $-10--5$ \\
$5-10$ & $-15--10$ \\
$0-5$ & $\leq-15$
\end{tabular}

Myanmar 
Average Percent Tree Cover by Province, and Areal Extent of Tree Cover Classes $\left(\mathrm{km}^{2}\right)$

\begin{tabular}{|c|c|c|c|c|c|c|c|c|c|c|c|c|c|c|c|c|}
\hline \multicolumn{2}{|c|}{ Nepal } & \multirow{3}{*}{\multicolumn{3}{|c|}{\begin{tabular}{|c|} 
Average Tree Cover by Province \\
Mean Tree Cover Percent by Province \\
$(\%)$
\end{tabular}}} & \multicolumn{12}{|c|}{ Areal Extent of Tree Cover Class $\left(\mathrm{km}^{2}\right)$} \\
\hline \multirow{3}{*}{ Province } & \multirow{3}{*}{ Area_km } & & & & \multicolumn{4}{|c|}{ Year 2000} & \multicolumn{4}{|c|}{ Year 2010} & \multirow{2}{*}{\multicolumn{4}{|c|}{$\begin{array}{c}\text { Change }(2000 \text { to } 2010) \\
\text { Area by Tree Cover Class }\left(\mathrm{km}^{2}\right)\end{array}$}} \\
\hline & & & & & & ea by Tree & ver Class $(k$ & & & a by Tree & ver Class & & & & & \\
\hline & & 2000 & 2010 & Change & $<10 \%$ & $10-20 \%$ & $20-30 \%$ & $>30 \%$ & $<10 \%$ & $10-20 \%$ & $20-30 \%$ & $>30 \%$ & $<10 \%$ & $10-20 \%$ & $20-30 \%$ & $>30 \%$ \\
\hline Central & 11,611 & 17.44 & 16.70 & -0.75 & 4,210 & 3,367 & 2,446 & 1,588 & 4,667 & 2,920 & 2,631 & 1,393 & 457 & -447 & 185 & -195 \\
\hline East & 11,186 & 17.18 & 20.47 & 3.29 & 4,889 & 2,616 & 1,795 & 1,886 & 3,468 & 3,159 & 2,188 & 2,371 & $-1,421$ & 543 & 393 & 485 \\
\hline Far-Western & 6,651 & 20.08 & 19.63 & -0.44 & 1,827 & 1,977 & 1,559 & 1,288 & 1,962 & 1,741 & 1,852 & 1,096 & 135 & -236 & 293 & -192 \\
\hline Mid-Western & 10,383 & 19.07 & 18.02 & -1.04 & 3,277 & 2,868 & 2,327 & 1,911 & 3,495 & 2,706 & 2,751 & 1,431 & 218 & -162 & 424 & -480 \\
\hline West & 9,508 & 20.13 & 19.36 & -0.78 & 2,742 & 2,786 & 2,137 & 1,843 & 3,087 & 2,305 & 2,644 & 1,472 & 345 & -481 & 507 & -371 \\
\hline Nepal - All & 49,339 & 18.60 & 18.74 & 0.14 & 16,945 & 13,614 & 10,264 & 8,516 & 16,679 & 12,831 & 12,066 & 7,763 & -266 & -783 & 1,802 & -753 \\
\hline
\end{tabular}

Average Percent Tree Cover by Province, and Areal Extent of Tree Cover Classes as a Percent of the Total Agricultural Area (\%)

\begin{tabular}{|c|c|c|c|c|c|c|c|c|c|c|c|c|c|c|c|c|}
\hline \multicolumn{2}{|c|}{ Nepal } & \multirow{3}{*}{\multicolumn{3}{|c|}{$\begin{array}{c}\text { Average Tree Cover by Province } \\
\text { Percent Tree Cover } \\
(\%)\end{array}$}} & \multicolumn{12}{|c|}{ Areal Extent of Tree Cover Class as a Percent of Total Agricultural Area (\%) } \\
\hline \multirow{3}{*}{ Province } & \multirow{3}{*}{ Area_km } & & & & \multirow{2}{*}{\multicolumn{4}{|c|}{$\begin{array}{c}\text { Year } 2000 \\
\text { Percent of Total Agrciultural Area (\%) }\end{array}$}} & \multirow{2}{*}{\multicolumn{4}{|c|}{$\begin{array}{c}\text { Year 2010 } \\
\text { Percent of Total Agrciultural Area (\%) }\end{array}$}} & \multirow{2}{*}{\multicolumn{4}{|c|}{$\begin{array}{c}\text { Change (2000 to 2010) } \\
\text { Percent of Total Agrciultural Area (\%) }\end{array}$}} \\
\hline & & & & & & & & & & & & & & & & \\
\hline & & 2000 & 2010 & Change & $<10 \%$ & $10-20 \%$ & $20-30 \%$ & $>30 \%$ & $<10 \%$ & $10-20 \%$ & $20-30 \%$ & $>30 \%$ & $<10 \%$ & $10-20 \%$ & $20-30 \%$ & $>30 \%$ \\
\hline Central & 11,611 & 17.44 & 16.70 & -0.75 & 36.26 & 29.00 & 21.07 & 13.68 & 40.19 & 25.15 & 22.66 & 12.00 & 3.94 & -3.85 & 1.59 & -1.68 \\
\hline East & 11,186 & 17.18 & 20.47 & 3.29 & 43.71 & 23.39 & 16.05 & 16.86 & 31.00 & 28.24 & 19.56 & 21.20 & -12.70 & 4.85 & 3.51 & 4.34 \\
\hline Far-Western & 6,651 & 20.08 & 19.63 & -0.44 & 27.47 & 29.73 & 23.44 & 19.37 & 29.50 & 26.18 & 27.85 & 16.48 & 2.03 & -3.55 & 4.41 & -2.89 \\
\hline Mid-Western & 10,383 & 19.07 & 18.02 & -1.04 & 31.56 & 27.62 & 22.41 & 18.41 & 33.66 & 26.06 & 26.50 & 13.78 & 2.10 & -1.56 & 4.08 & -4.62 \\
\hline West & 9,508 & 20.13 & 19.36 & -0.78 & 28.84 & 29.30 & 22.48 & 19.38 & 32.47 & 24.24 & 27.81 & 15.48 & 3.63 & -5.06 & 5.33 & -3.90 \\
\hline Nepal - All & 49,339 & 18.60 & 18.74 & 0.14 & 34.34 & 27.59 & 20.80 & 17.26 & 33.81 & 26.01 & 24.46 & 15.73 & -0.54 & -1.59 & 3.65 & -1.53 \\
\hline
\end{tabular}


Asia Pacific Region : Tree Cover on Agricultural Land (2010)

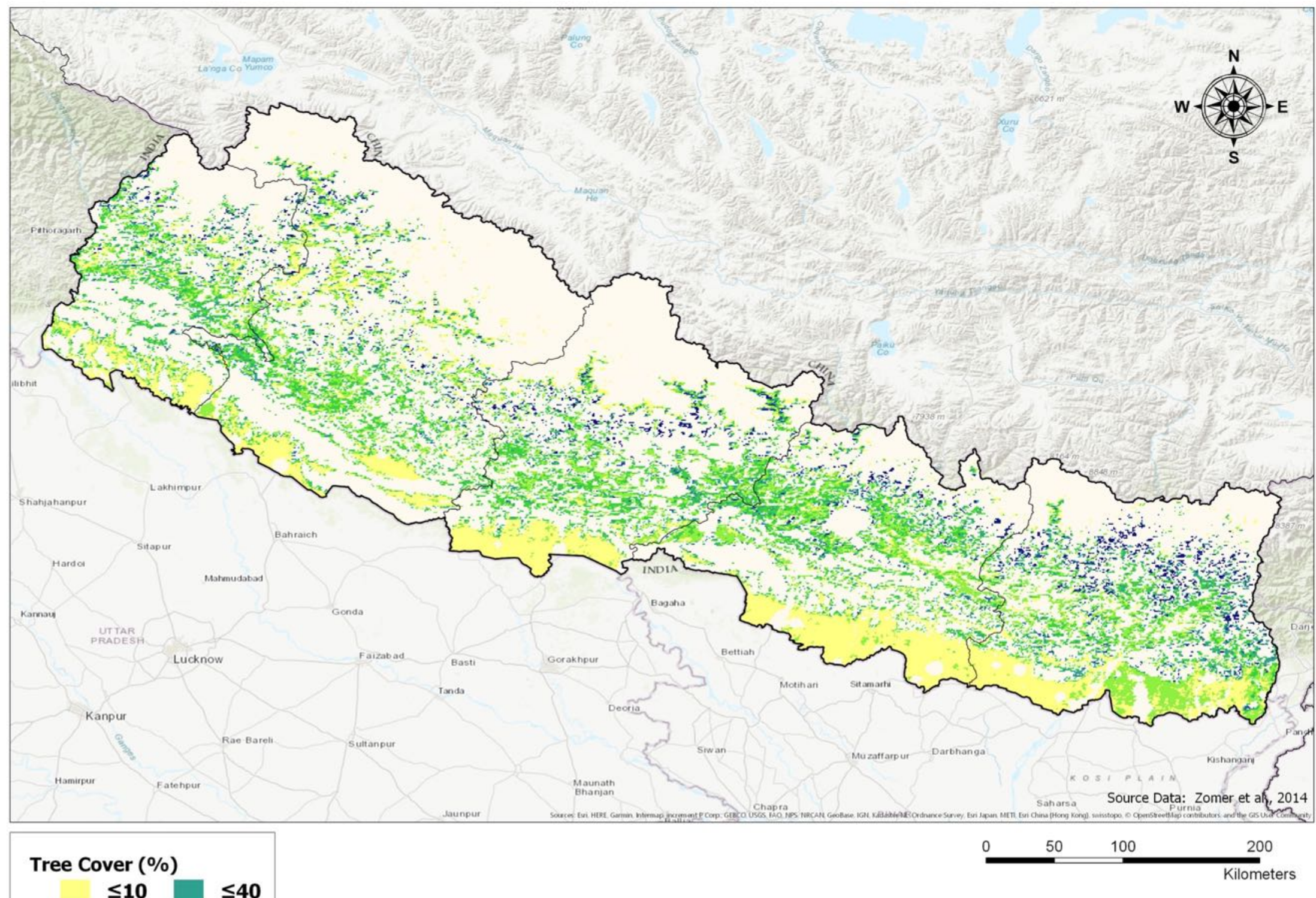

Nepal 
Asia Pacific Region : Change in Tree Cover on Agricultural Land (2000 - 2010)

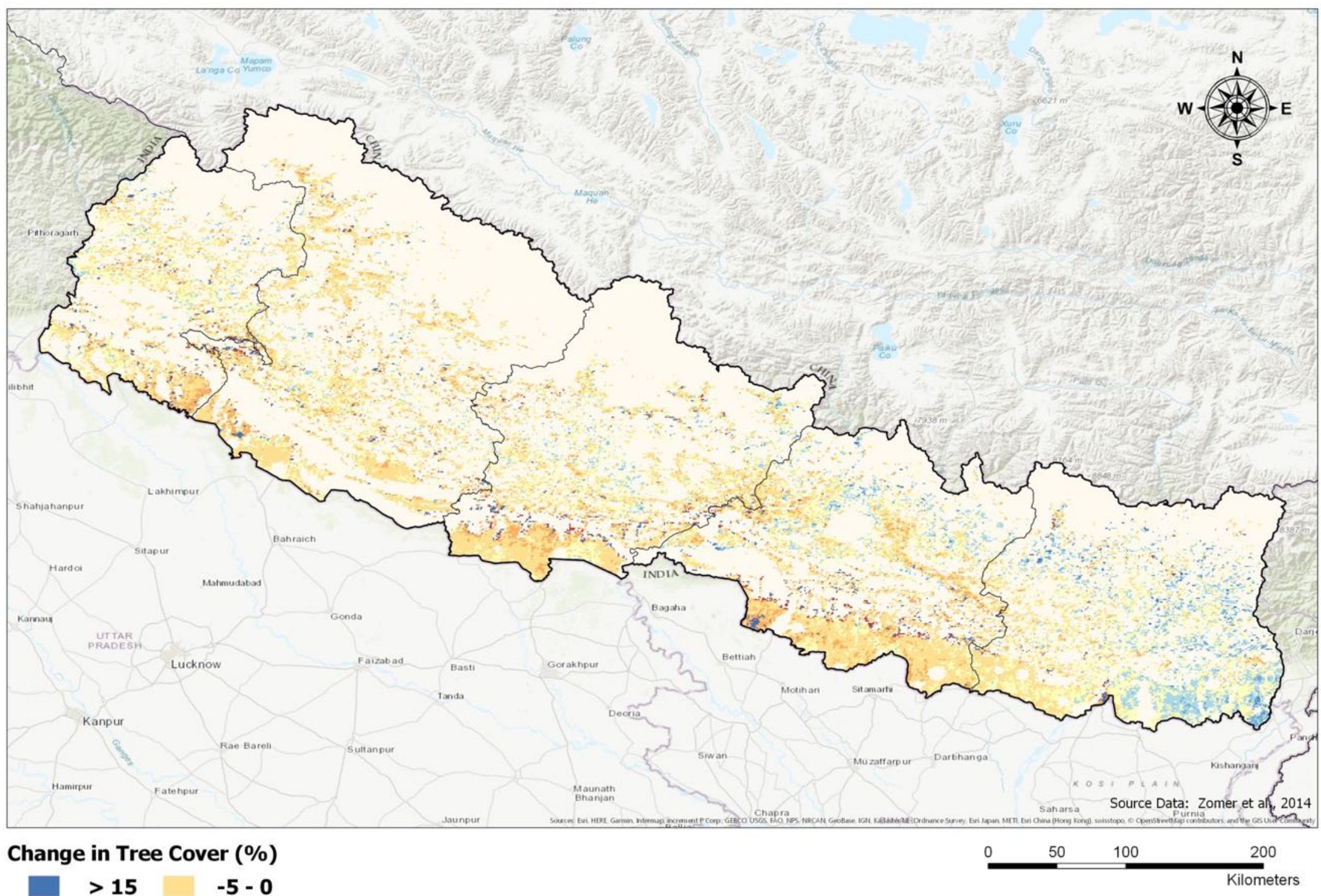

\begin{tabular}{|r|r|}
\hline$>15$ & $-5-0$ \\
$10-15$ & $-10--5$ \\
$5-10$ & $-15--10$ \\
$0-5$ & $\leq-15$
\end{tabular}

Nepal 
Average Percent Tree Cover by Province, and Areal Extent of Tree Cover Classes $\left(\mathrm{km}^{2}\right)$

\begin{tabular}{|c|c|c|c|c|c|c|c|c|c|c|c|c|c|c|c|c|}
\hline \multicolumn{2}{|c|}{ New Zealand } & \multirow{3}{*}{\multicolumn{3}{|c|}{\begin{tabular}{|c|} 
Average Tree Cover by Province \\
Mean Tree Cover Percent by Province \\
$(\%)$
\end{tabular}}} & \multicolumn{12}{|c|}{ Areal Extent of Tree Cover Class $\left(\mathrm{km}^{2}\right)$} \\
\hline \multirow{3}{*}{ Province } & \multirow{3}{*}{ Area_km } & & & & \multicolumn{4}{|c|}{ Year 2000} & \multicolumn{4}{|c|}{ Year 2010} & \multirow{2}{*}{\multicolumn{4}{|c|}{ Change (2000 to 2010) }} \\
\hline & & & & & & ea by Tree & ver Class $(k$ & & & rea by Tree & over Class & & & & & \\
\hline & & 2000 & 2010 & Change & $<10 \%$ & $10-20 \%$ & $20-30 \%$ & $>30 \%$ & $<10 \%$ & $10-20 \%$ & $20-30 \%$ & $>30 \%$ & $<10 \%$ & $10-20 \%$ & $20-30 \%$ & $>30 \%$ \\
\hline Auckland & 1,504 & 34.51 & 40.77 & 6.26 & 4 & 108 & 482 & 910 & 2 & 14 & 226 & 1,262 & -2 & -94 & -256 & 352 \\
\hline Bay of Plenty & 1,536 & 34.00 & 35.89 & 1.89 & 4 & 80 & 540 & 912 & 0 & 53 & 471 & 1,012 & -4 & -27 & -69 & 100 \\
\hline Canterbury & 14,733 & 13.90 & 21.17 & 7.27 & 5,684 & 6,819 & 1,699 & 531 & 2,148 & 5,743 & 4,263 & 2,579 & $-3,536$ & $-1,076$ & 2,564 & 2,048 \\
\hline Gisborne & 2,120 & 24.94 & 30.11 & 5.16 & 38 & 793 & 888 & 401 & 7 & 311 & 1,095 & 707 & -31 & -482 & 207 & 306 \\
\hline Hawke's Bay & 5,352 & 25.70 & 28.69 & 2.99 & 52 & 1,759 & 2,302 & 1,239 & 3 & 960 & 2,906 & 1,483 & -49 & -799 & 604 & 244 \\
\hline Manawatu-Wanganui & 10,855 & 25.90 & 31.98 & 6.08 & 53 & 3,659 & 4,715 & 2,428 & 2 & 407 & 5,724 & 4,722 & -51 & $-3,252$ & 1,009 & 2,294 \\
\hline Marlborough & 687 & 10.61 & 18.02 & 7.41 & 465 & 175 & 31 & 16 & 99 & 409 & 123 & 56 & -366 & 234 & 92 & 40 \\
\hline Northland & 5,107 & 35.57 & 37.04 & 1.47 & 25 & 418 & 1,758 & 2,906 & 19 & 211 & 1,692 & 3,185 & -6 & -207 & -66 & 279 \\
\hline Otago & 9,687 & 13.76 & 19.50 & 5.74 & 4,697 & 3,062 & 1,516 & 412 & 2,413 & 3,625 & 1,982 & 1,667 & $-2,284$ & 563 & 466 & 1,255 \\
\hline Southland & 8,779 & 22.15 & 31.48 & 9.32 & 466 & 3,928 & 3,207 & 1,178 & 56 & 1,356 & 3,218 & 4,149 & -410 & $-2,572$ & 11 & 2,971 \\
\hline Taranaki & 2,846 & 37.66 & 42.64 & 4.98 & 0 & 192 & 475 & 2,179 & 2 & 10 & 414 & 2,420 & 2 & -182 & -61 & 241 \\
\hline Tasman & 149 & 31.97 & 36.63 & 4.66 & 0 & 29 & 47 & 73 & 0 & 9 & 45 & 95 & 0 & -20 & -2 & 22 \\
\hline Waikato & 10,305 & 32.91 & 37.24 & 4.33 & 79 & 827 & 3,377 & 6,022 & 2 & 381 & 2,044 & 7,878 & -77 & -446 & $-1,333$ & 1,856 \\
\hline Wellington & 2,746 & 27.39 & 30.42 & 3.03 & 0 & 783 & 1,079 & 884 & 0 & 159 & 1,614 & 973 & 0 & -624 & 535 & 89 \\
\hline West Coast & 352 & 32.67 & 39.63 & 6.96 & 3 & 44 & 127 & 178 & 1 & 13 & 69 & 269 & -2 & -31 & -58 & 91 \\
\hline New Zealand - All & 76,758 & 23.91 & 29.54 & 5.64 & 11,570 & 22,676 & 22,243 & 20,269 & 4,754 & 13,661 & 25,886 & 32,457 & $-6,816$ & $-9,015$ & 3,643 & 12,188 \\
\hline
\end{tabular}

Average Percent Tree Cover by Province, and Areal Extent of Tree Cover Classes as a Percent of the Total Agricultural Area (\%)

\begin{tabular}{|c|c|c|c|c|c|c|c|c|c|c|c|c|c|c|c|c|}
\hline \multicolumn{2}{|c|}{ New Zealand } & \multirow{3}{*}{\multicolumn{3}{|c|}{$\begin{array}{c}\text { Average Tree Cover by Province } \\
\text { Percent Tree Cover } \\
(\%)\end{array}$}} & \multicolumn{12}{|c|}{ Areal Extent of Tree Cover Class as a Percent of Total Agricultural Area (\%) } \\
\hline \multirow{3}{*}{ Province } & \multirow{3}{*}{ Area_km } & & & & \multirow{2}{*}{\multicolumn{4}{|c|}{$\begin{array}{c}\text { Year } 2000 \\
\text { Percent of Total Agrciultural Area }(\%)\end{array}$}} & \multirow{2}{*}{\multicolumn{4}{|c|}{$\begin{array}{c}\text { Year } 2010 \\
\text { Percent of Total Agrciultural Area (\%) }\end{array}$}} & \multirow{2}{*}{\multicolumn{4}{|c|}{$\begin{array}{c}\text { Change (2000 to 2010) } \\
\text { Percent of Total Agrciultural Area (\%) }\end{array}$}} \\
\hline & & & & & & & & & & & & & & & & \\
\hline & & 2000 & 2010 & Change & $<10 \%$ & $10-20 \%$ & $20-30 \%$ & $>30 \%$ & $<10 \%$ & $10-20 \%$ & $20-30 \%$ & $>30 \%$ & $<10 \%$ & $10-20 \%$ & $20-30 \%$ & $>30 \%$ \\
\hline Auckland & 1,504 & 34.51 & 40.77 & 6.26 & 0.27 & 7.18 & 32.05 & 60.51 & 0.13 & 0.93 & 15.03 & 83.91 & -0.13 & -6.25 & -17.02 & 23.40 \\
\hline Bay of Plenty & 1,536 & 34.00 & 35.89 & 1.89 & 0.26 & 5.21 & 35.16 & 59.38 & 0.00 & 3.45 & 30.66 & 65.89 & -0.26 & -1.76 & -4.49 & 6.51 \\
\hline Canterbury & 14,733 & 13.90 & 21.17 & 7.27 & 38.58 & 46.28 & 11.53 & 3.60 & 14.58 & 38.98 & 28.94 & 17.51 & -24.00 & -7.30 & 17.40 & 13.90 \\
\hline Gisborne & 2,120 & 24.94 & 30.11 & 5.16 & 1.79 & 37.41 & 41.89 & 18.92 & 0.33 & 14.67 & 51.65 & 33.35 & -1.46 & -22.74 & 9.76 & 14.43 \\
\hline Hawke's Bay & 5,352 & 25.70 & 28.69 & 2.99 & 0.97 & 32.87 & 43.01 & 23.15 & 0.06 & 17.94 & 54.30 & 27.71 & -0.92 & -14.93 & 11.29 & 4.56 \\
\hline Manawatu-Wanganui & 10,855 & 25.90 & 31.98 & 6.08 & 0.49 & 33.71 & 43.44 & 22.37 & 0.02 & 3.75 & 52.73 & 43.50 & -0.47 & -29.96 & 9.30 & 21.13 \\
\hline Marlborough & 687 & 10.61 & 18.02 & 7.41 & 67.69 & 25.47 & 4.51 & 2.33 & 14.41 & 59.53 & 17.90 & 8.15 & -53.28 & 34.06 & 13.39 & 5.82 \\
\hline Northland & 5,107 & 35.57 & 37.04 & 1.47 & 0.49 & 8.18 & 34.42 & 56.90 & 0.37 & 4.13 & 33.13 & 62.37 & -0.12 & -4.05 & -1.29 & 5.46 \\
\hline Otago & 9,687 & 13.76 & 19.50 & 5.74 & 48.49 & 31.61 & 15.65 & 4.25 & 24.91 & 37.42 & 20.46 & 17.21 & -23.58 & 5.81 & 4.81 & 12.96 \\
\hline Southland & 8,779 & 22.15 & 31.48 & 9.32 & 5.31 & 44.74 & 36.53 & 13.42 & 0.64 & 15.45 & 36.66 & 47.26 & -4.67 & -29.30 & 0.13 & 33.84 \\
\hline Taranaki & 2,846 & 37.66 & 42.64 & 4.98 & 0.00 & 6.75 & 16.69 & 76.56 & 0.07 & 0.35 & 14.55 & 85.03 & 0.07 & -6.39 & -2.14 & 8.47 \\
\hline Tasman & 149 & 31.97 & 36.63 & 4.66 & 0.00 & 19.46 & 31.54 & 48.99 & 0.00 & 6.04 & 30.20 & 63.76 & 0.00 & -13.42 & -1.34 & 14.77 \\
\hline Waikato & 10,305 & 32.91 & 37.24 & 4.33 & 0.77 & 8.03 & 32.77 & 58.44 & 0.02 & 3.70 & 19.84 & 76.45 & -0.75 & -4.33 & -12.94 & 18.01 \\
\hline Wellington & 2,746 & 27.39 & 30.42 & 3.03 & 0.00 & 28.51 & 39.29 & 32.19 & 0.00 & 5.79 & 58.78 & 35.43 & 0.00 & -22.72 & 19.48 & 3.24 \\
\hline West Coast & 352 & 32.67 & 39.63 & 6.96 & 0.85 & 12.50 & 36.08 & 50.57 & 0.28 & 3.69 & 19.60 & 76.42 & -0.57 & -8.81 & -16.48 & 25.85 \\
\hline New Zealand - All & 76,758 & 23.91 & 29.54 & 5.64 & 15.07 & 29.54 & 28.98 & 26.41 & 6.19 & 17.80 & 33.72 & 42.29 & -8.88 & -11.74 & 4.75 & 15.88 \\
\hline
\end{tabular}


Asia Pacific Region : Tree Cover on Agricultural Land (2010)

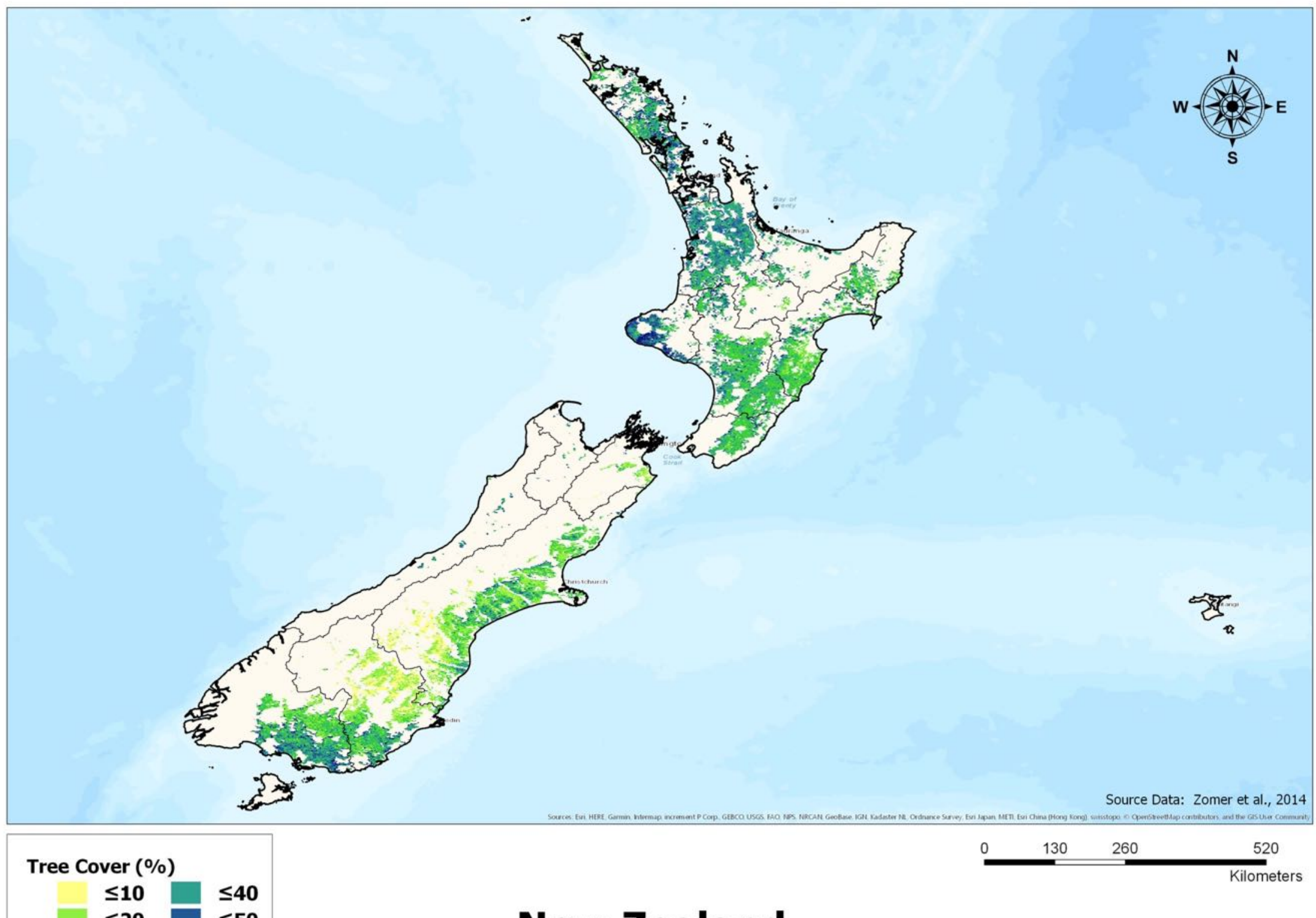

New Zealand 
Asia Pacific Region : Change in Tree Cover on Agricultural Land (2000 - 2010)

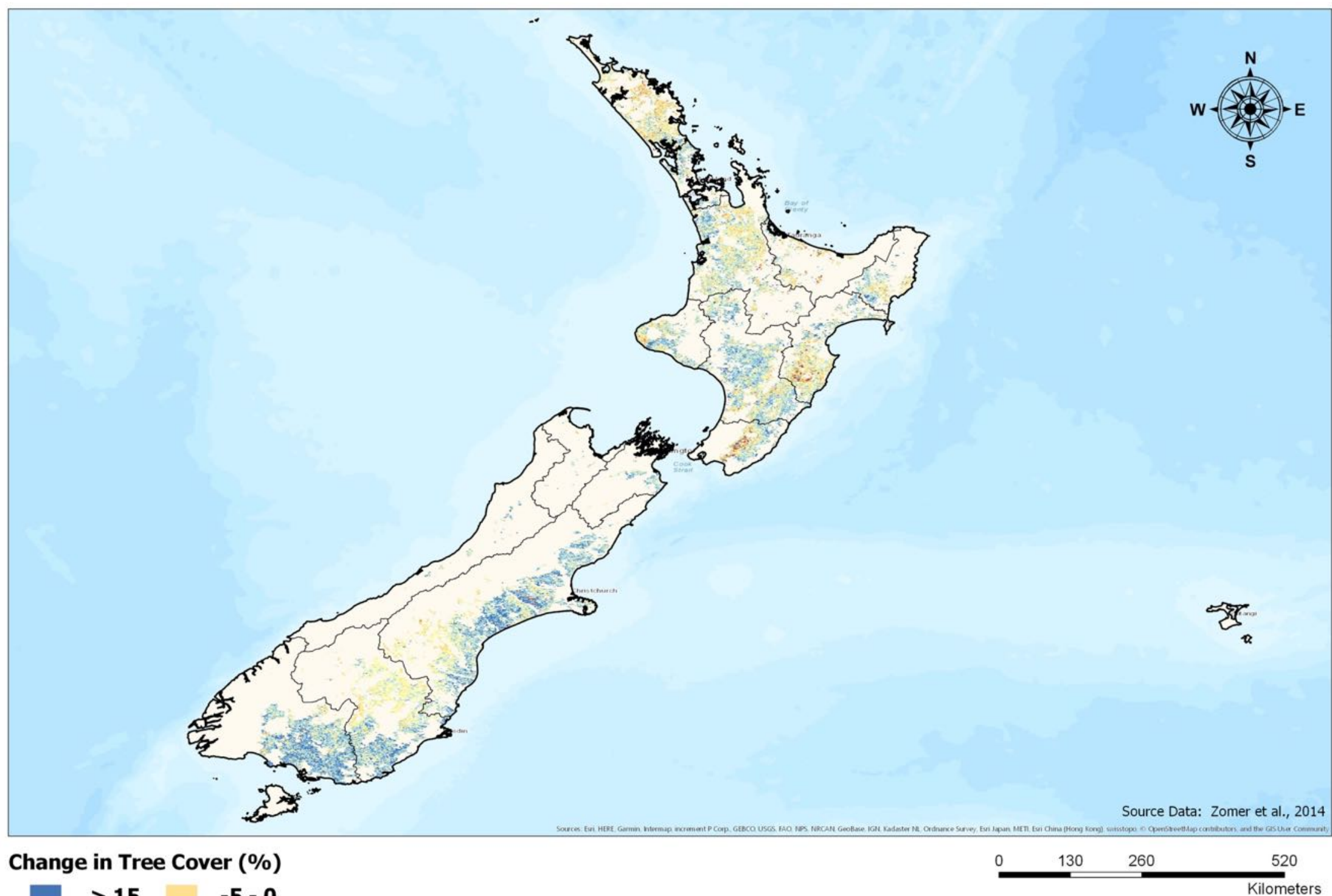

$>15 \square-5-0$

$10-15-10--5$

$5-10-15--10$

New Zealand

$0-5 \square \leq-15$ 


\section{Appendix 17: North Korea}

Average Percent Tree Cover by Province, and Areal Extent of Tree Cover Classes $\left(\mathrm{km}^{2}\right)$

\begin{tabular}{|c|c|c|c|c|c|c|c|c|c|c|c|c|c|c|c|c|}
\hline \multicolumn{2}{|c|}{ North Korea } & \multirow{2}{*}{\multicolumn{3}{|c|}{\begin{tabular}{|c|} 
Average Tree Cover by Province \\
Mean Tree Cover Percent by Province \\
\end{tabular}}} & \multicolumn{12}{|c|}{ Areal Extent of Tree Cover Class $\left(\mathrm{km}^{2}\right)$} \\
\hline \multirow{3}{*}{ Province } & \multirow{3}{*}{ Area_km } & & & & \multicolumn{4}{|c|}{ Year 2000} & \multicolumn{4}{|c|}{ Year 2010} & \multicolumn{4}{|c|}{ Change (2000 to 2010) } \\
\hline & & & (\%) & & & a by Tree $\mathrm{C}$ & ver Class (kr & & & ea by Tree C & over Class (k & & & ea by Tree & over Class ( & \\
\hline & & 2000 & 2010 & Change & $<10 \%$ & $10-20 \%$ & $20-30 \%$ & $>30 \%$ & $<10 \%$ & $10-20 \%$ & $20-30 \%$ & $>30 \%$ & $<10 \%$ & $10-20 \%$ & $20-30 \%$ & $>30 \%$ \\
\hline Chagang-do & 540 & 32.84 & 31.00 & -1.83 & 140 & 108 & 57 & 235 & 134 & 128 & 48 & 230 & -6 & 20 & -9 & -5 \\
\hline Hamgyŏng-bukto & 1,773 & 27.10 & 26.15 & -0.95 & 579 & 347 & 199 & 648 & 686 & 273 & 176 & 638 & 107 & -74 & -23 & -10 \\
\hline Hamgyŏng-namdo & 2,361 & 18.57 & 18.44 & -0.13 & 1,060 & 578 & 267 & 456 & 1,149 & 482 & 248 & 482 & 89 & -96 & -19 & 26 \\
\hline Hwanghae-bukto & 2,599 & 9.09 & 9.08 & -0.01 & 2,031 & 393 & 101 & 74 & 1,959 & 473 & 86 & 81 & -72 & 80 & -15 & 7 \\
\hline Hwanghae-namdo & 5,320 & 8.54 & 9.02 & 0.48 & 4,031 & 922 & 233 & 134 & 3,946 & 902 & 291 & 181 & -85 & -20 & 58 & 47 \\
\hline Kaesǒng & 443 & 6.42 & 8.09 & 1.67 & 399 & 41 & 3 & 0 & 349 & 81 & 8 & 5 & -50 & 40 & 5 & 5 \\
\hline Kangwŏn-do & 925 & 15.92 & 15.55 & -0.37 & 475 & 244 & 89 & 117 & 480 & 269 & 63 & 113 & 5 & 25 & -26 & -4 \\
\hline Kumgangsan & 21 & 34.29 & 30.00 & -4.29 & 10 & 0 & 0 & 11 & 10 & 0 & 0 & 11 & 0 & 0 & 0 & 0 \\
\hline P'yŏngan-bukto & 2,005 & 9.83 & 11.28 & 1.44 & 1,510 & 308 & 87 & 100 & 1,233 & 571 & 94 & 107 & -277 & 263 & 7 & 7 \\
\hline P'yŏngan-namdo & 4,284 & 12.33 & 11.98 & -0.35 & 2,651 & 1,054 & 299 & 280 & 2,457 & 1,373 & 214 & 240 & -194 & 319 & -85 & -40 \\
\hline P'yŏngyang & 1,659 & 13.54 & 12.81 & -0.73 & 873 & 475 & 214 & 97 & 867 & 556 & 160 & 76 & -6 & 81 & -54 & -21 \\
\hline Rasǒn & 101 & 17.52 & 17.12 & -0.41 & 46 & 22 & 13 & 20 & 43 & 20 & 19 & 19 & -3 & -2 & 6 & -1 \\
\hline Ryanggang & 610 & 44.51 & 44.16 & -0.35 & 65 & 70 & 31 & 444 & 56 & 72 & 33 & 449 & -9 & 2 & 2 & 5 \\
\hline Sinǔiju & 19 & 10.79 & 12.63 & 1.84 & 8 & 11 & 0 & 0 & 4 & 15 & 0 & 0 & -4 & 4 & 0 & 0 \\
\hline North Korea - All & 22,660 & 14.17 & 14.16 & -0.01 & 13,878 & 4,573 & 1,593 & 2,616 & 13,373 & 5,215 & 1,440 & 2,632 & -505 & 642 & -153 & 16 \\
\hline
\end{tabular}

Average Percent Tree Cover by Province, and Areal Extent of Tree Cover Classes as a Percent of the Total Agricultural Area (\%)

\begin{tabular}{|c|c|c|c|c|c|c|c|c|c|c|c|c|c|c|c|c|}
\hline \multicolumn{2}{|c|}{ North Korea } & \multirow{3}{*}{\multicolumn{3}{|c|}{$\begin{array}{c}\text { Average Tree Cover by Province } \\
\text { Percent Tree Cover } \\
(\%)\end{array}$}} & \multicolumn{12}{|c|}{ Areal Extent of Tree Cover Class as a Percent of Total Agricultural Area (\%) } \\
\hline \multirow{2}{*}{ Province } & \multirow{2}{*}{ Area_km } & & & & \multirow{2}{*}{\multicolumn{4}{|c|}{$\begin{array}{c}\text { Year } 2000 \\
\text { Percent of Total Agrciultural Area (\%) }\end{array}$}} & Perc & $\begin{aligned} Y e \\
\end{aligned}$ & & & & Change (2 & 0 to 2010) & \\
\hline & & & & & $<10 \%$ & $10-20 \%$ & & & \multicolumn{4}{|c|}{ Percent of Total Agrciultural Area (\%) } & \multicolumn{4}{|c|}{ Percent of Total Agrciultural Area (\%) } \\
\hline Chagang-do & 540 & 3284 & 3100 & -1.83 & 25.93 & 20.00 & 10.56 & 4352 & 2482 & 2370 & $\frac{20.010}{809}$ & & 111 & & $20-30 \%$ & $>30 \%$ \\
\hline Hamgyǒng-bukto & 1,773 & 27.10 & 26.15 & -0.95 & 32.66 & 19.57 & 11.22 & 36.55 & 38.69 & 15.40 & 9.93 & 3508 & 604 & -4.17 & $\begin{array}{l}-1.01 \\
-1.30\end{array}$ & -0.93 \\
\hline Hamgyŏng-namdo & 2,361 & 18.57 & 18.44 & -0.13 & 44.90 & 24.48 & 11.31 & 19.31 & 48.67 & 20.42 & 10.50 & 20.42 & 3.77 & -4.07 & -0.80 & 1.10 \\
\hline Hwanghae-bukto & 2,599 & 9.09 & 9.08 & -0.01 & 78.15 & 15.12 & 3.89 & 2.85 & 75.38 & 18.20 & 3.31 & 3.12 & -2.77 & 3.08 & -0.58 & 0.27 \\
\hline Hwanghae-namdo & 5,320 & 8.54 & 9.02 & 0.48 & 75.77 & 17.33 & 4.38 & 2.52 & 74.17 & 16.96 & 5.47 & 3.40 & -1.60 & -0.38 & 1.09 & 0.88 \\
\hline Kaesǒng & 443 & 6.42 & 8.09 & 1.67 & 90.07 & 9.26 & 0.68 & 0.00 & 78.78 & 18.28 & 1.81 & 1.13 & -11.29 & 9.03 & 1.13 & 1.13 \\
\hline Kangwŏn-do & 925 & 15.92 & 15.55 & -0.37 & 51.35 & 26.38 & 9.62 & 12.65 & 51.89 & 29.08 & 6.81 & 12.22 & 0.54 & 2.70 & -2.81 & -0.43 \\
\hline Kumgangsan & 21 & 34.29 & 30.00 & -4.29 & 47.62 & 0.00 & 0.00 & 52.38 & 47.62 & 0.00 & 0.00 & 52.38 & 0.00 & 0.00 & 0.00 & 0.00 \\
\hline P'yŏngan-bukto & 2,005 & 9.83 & 11.28 & 1.44 & 75.31 & 15.36 & 4.34 & 4.99 & 61.50 & 28.48 & 4.69 & 5.34 & -13.82 & 13.12 & 0.35 & 0.35 \\
\hline P'yŏngan-namdo & 4,284 & 12.33 & 11.98 & -0.35 & 61.88 & 24.60 & 6.98 & 6.54 & 57.35 & 32.05 & 5.00 & 5.60 & -4.53 & 7.45 & -1.98 & -0.93 \\
\hline P'yŏngyang & 1,659 & 13.54 & 12.81 & -0.73 & 52.62 & 28.63 & 12.90 & 5.85 & 52.26 & 33.51 & 9.64 & 4.58 & -0.36 & 4.88 & -3.25 & -1.27 \\
\hline Rasǒn & 101 & 17.52 & 17.12 & -0.41 & 45.54 & 21.78 & 12.87 & 19.80 & 42.57 & 19.80 & 18.81 & 18.81 & -2.97 & -1.98 & 5.94 & -0.99 \\
\hline Ryanggang & 610 & 44.51 & 44.16 & -0.35 & 10.66 & 11.48 & 5.08 & 72.79 & 9.18 & 11.80 & 5.41 & 73.61 & -1.48 & 0.33 & 0.33 & 0.82 \\
\hline Sinǔiju & 19 & 10.79 & 12.63 & 1.84 & 42.11 & 57.90 & 0.00 & 0.00 & 21.05 & 78.95 & 0.00 & 0.00 & -21.05 & 21.05 & 0.00 & 0.00 \\
\hline North Korea - All & 22,660 & 14.17 & 14.16 & -0.01 & 61.24 & 20.18 & 7.03 & 11.54 & 59.02 & 23.01 & 6.35 & 11.62 & -2.23 & 2.83 & -0.68 & 0.07 \\
\hline
\end{tabular}


Asia Pacific Region : Tree Cover on Agricultural Land (2010)

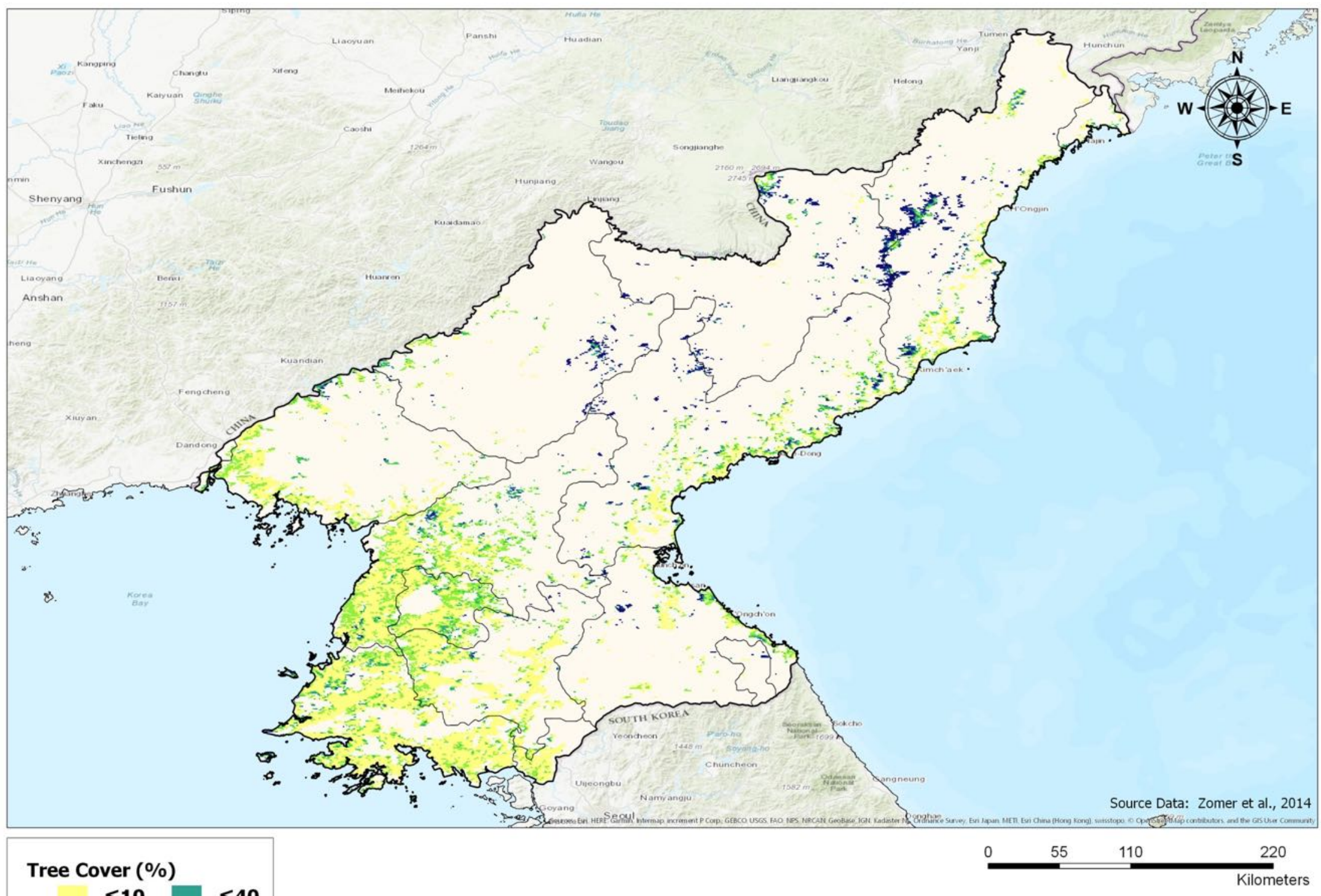

North Korea 
Asia Pacific Region : Change in Tree Cover on Agricultural Land (2000 - 2010)

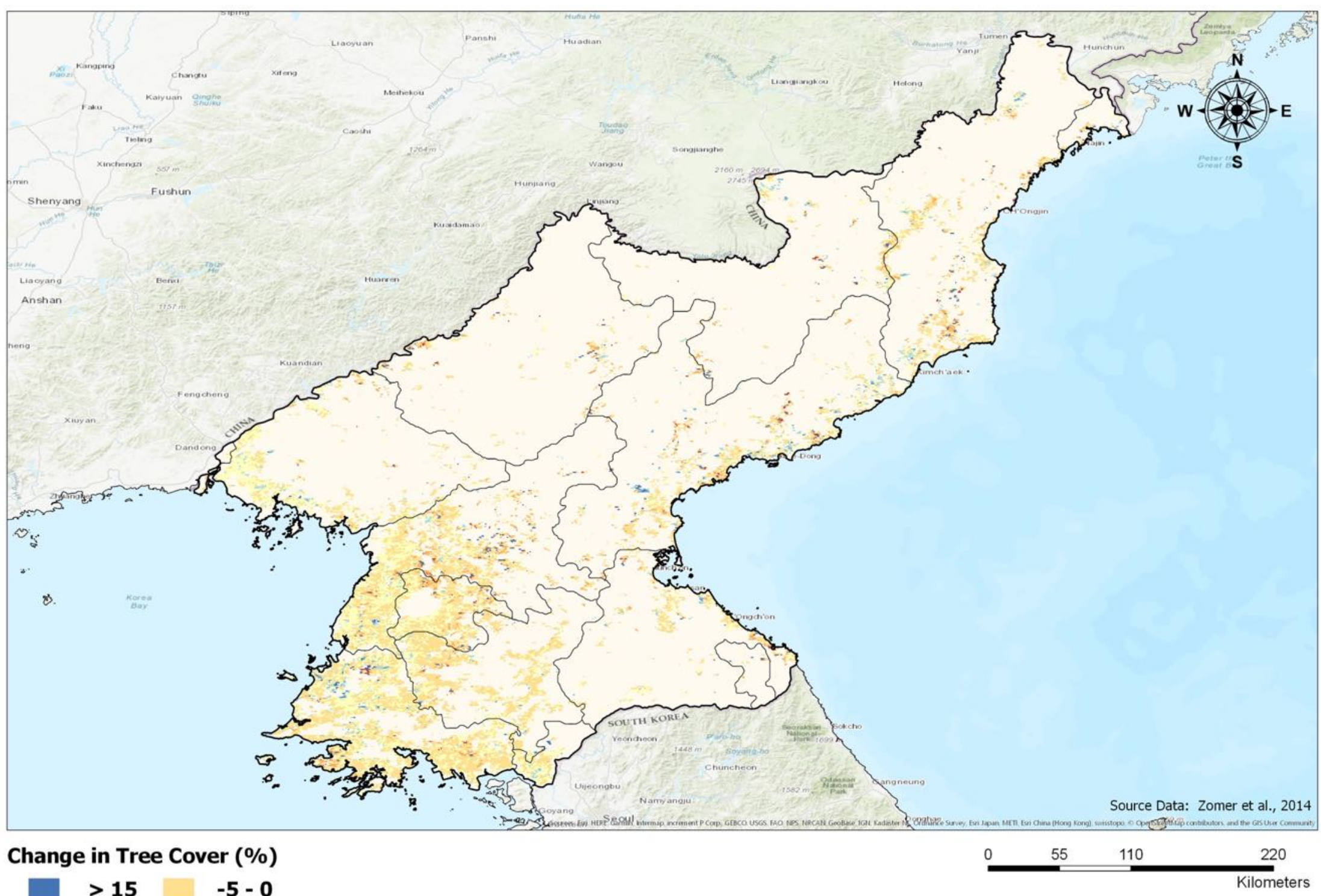

\begin{tabular}{|r|r|}
\hline$>15$ & $-5-0$ \\
$10-15$ & $-10--5$ \\
$5-10$ & $-15--10$ \\
$0-5$ & $\leq-15$
\end{tabular}

North Korea 
Average Percent Tree Cover by Province, and Areal Extent of Tree Cover Classes $\left(\mathrm{km}^{2}\right)$

\begin{tabular}{|c|c|c|c|c|c|c|c|c|c|c|c|c|c|c|c|c|}
\hline \multicolumn{2}{|c|}{ Pakistan } & \multirow{3}{*}{\multicolumn{3}{|c|}{\begin{tabular}{|c|} 
Average Tree Cover by Province \\
Mean Tree Cover Percent by Province \\
\end{tabular}}} & \multicolumn{12}{|c|}{ Areal Extent of Tree Cover Class $\left(\mathrm{km}^{2}\right)$} \\
\hline \multirow{3}{*}{ Province } & \multirow{3}{*}{ Area_km } & & & & \multirow{2}{*}{\multicolumn{4}{|c|}{$\begin{array}{c}\text { Year } 2000 \\
\text { Area by Tree Cover Class }\left(\mathrm{km}^{2}\right)\end{array}$}} & \multirow{2}{*}{\multicolumn{4}{|c|}{$\begin{array}{c}\text { Year } 2010 \\
\text { Area by Tree Cover Class }\left(\mathrm{km}^{2}\right)\end{array}$}} & \multirow{2}{*}{\multicolumn{4}{|c|}{ Change (2000 to 2010) }} \\
\hline & & & & & & & ver Class $(k$ & & & & & & & & & \\
\hline & & 2000 & 2010 & Change & $<10 \%$ & $10-20 \%$ & $20-30 \%$ & $>30 \%$ & $<10 \%$ & $10-20 \%$ & $20-30 \%$ & $>30 \%$ & $<10 \%$ & $10-20 \%$ & $20-30 \%$ & $>30 \%$ \\
\hline Azad Kashmir & 6,484 & 15.60 & 18.00 & 2.41 & 2,163 & 2,734 & 1,283 & 304 & 1,658 & 2,418 & 1,752 & 656 & -505 & -316 & 469 & 352 \\
\hline Baluchistan & 32,500 & 0.37 & 0.70 & 0.33 & 32,479 & 21 & 0 & 0 & 32,452 & 48 & 0 & 0 & -27 & 27 & 0 & 0 \\
\hline F.A.T.A. & 8,782 & 3.06 & 4.92 & 1.87 & 8,020 & 565 & 161 & 36 & 7,718 & 775 & 228 & 61 & -302 & 210 & 67 & 25 \\
\hline $\begin{array}{l}\text { F.C.T. } \\
\text { F. }\end{array}$ & 290 & 6.69 & 7.07 & 0.38 & 245 & 35 & 10 & 0 & 240 & 32 & 15 & 3 & -5 & -3 & 5 & 3 \\
\hline N.W.F.P. & 24,067 & 5.45 & 7.22 & 1.77 & 19,832 & 3,011 & 909 & 315 & 18,192 & 4,038 & 1,337 & 500 & $-1,640$ & 1,027 & 428 & 185 \\
\hline Northern Areas & 992 & 6.56 & 7.46 & 0.90 & 811 & 117 & 47 & 17 & 787 & 113 & 71 & 21 & -24 & -4 & 24 & 4 \\
\hline Punjab & 135,330 & 4.33 & 4.78 & 0.45 & 130,684 & 4,374 & 254 & 19 & 130,769 & 4,103 & 357 & 102 & 85 & -271 & 103 & 83 \\
\hline Sind & 64,805 & 3.17 & 3.85 & 0.68 & 64,482 & 320 & 3 & 0 & 63,753 & 1,046 & 6 & 0 & -729 & 726 & 3 & 0 \\
\hline Pakistan - All & 273,250 & 3.92 & 4.62 & 0.70 & 258,717 & 11,177 & 2,667 & 691 & 255,570 & 12,573 & 3,766 & 1,343 & $-3,147$ & 1,396 & 1,099 & 652 \\
\hline
\end{tabular}

Average Percent Tree Cover by Province, and Areal Extent of Tree Cover Classes as a Percent of the Total Agricultural Area (\%)

\begin{tabular}{|c|c|c|c|c|c|c|c|c|c|c|c|c|c|c|c|c|}
\hline \multicolumn{2}{|c|}{ Pakistan } & \multirow{3}{*}{\multicolumn{3}{|c|}{$\begin{array}{c}\text { Average Tree Cover by Province } \\
\text { Percent Tree Cover } \\
(\%)\end{array}$}} & \multicolumn{12}{|c|}{ Areal Extent of Tree Cover Class as a Percent of Total Agricultural Area (\%) } \\
\hline \multirow{3}{*}{ Province } & \multirow{3}{*}{ Area_km } & & & & \multirow{2}{*}{\multicolumn{4}{|c|}{$\begin{array}{c}\text { Year 2000 } \\
\text { Percent of Total Agrciultural Area }(\%)\end{array}$}} & \multirow{2}{*}{\multicolumn{4}{|c|}{$\begin{array}{c}\text { Year } 2010 \\
\text { Percent of Total Agrciultural Area (\%) }\end{array}$}} & \multirow{2}{*}{\multicolumn{4}{|c|}{$\begin{array}{c}\text { Change (2000 to 2010) } \\
\text { Percent of Total Agrciultural Area (\%) }\end{array}$}} \\
\hline & & & & & & & & & & & & & & & & \\
\hline & & 2000 & 2010 & Change & $<10 \%$ & $10-20 \%$ & $20-30 \%$ & $>30 \%$ & $<10 \%$ & $10-20 \%$ & $20-30 \%$ & $>30 \%$ & $<10 \%$ & $10-20 \%$ & $20-30 \%$ & $>30 \%$ \\
\hline Azad Kashmir & 6,484 & 15.60 & 18.00 & 2.41 & 33.36 & 42.17 & 19.79 & 4.69 & 25.57 & 37.29 & 27.02 & 10.12 & -7.79 & -4.87 & 7.23 & 5.43 \\
\hline Baluchistan & 32,500 & 0.37 & 0.70 & 0.33 & 99.94 & 0.06 & 0.00 & 0.00 & 99.85 & 0.15 & 0.00 & 0.00 & -0.08 & 0.08 & 0.00 & 0.00 \\
\hline F.A.T.A. & 8,782 & 3.06 & 4.92 & 1.87 & 91.32 & 6.43 & 1.83 & 0.41 & 87.88 & 8.82 & 2.60 & 0.69 & -3.44 & 2.39 & 0.76 & 0.28 \\
\hline F.C.T. & 290 & 6.69 & 7.07 & 0.38 & 84.48 & 12.07 & 3.45 & 0.00 & 82.76 & 11.03 & 5.17 & 1.03 & -1.72 & -1.03 & 1.72 & 1.03 \\
\hline N.W.F.P & 24,067 & 5.45 & 7.22 & 1.77 & 82.40 & 12.51 & 3.78 & 1.31 & 75.59 & 16.78 & 5.56 & 2.08 & -6.81 & 4.27 & 1.78 & 0.77 \\
\hline Northern Areas & 992 & 6.56 & 7.46 & 0.90 & 81.75 & 11.79 & 4.74 & 1.71 & 79.34 & 11.39 & 7.16 & 2.12 & -2.42 & -0.40 & 2.42 & 0.40 \\
\hline Punjab & 135,330 & 4.33 & 4.78 & 0.45 & 96.57 & 3.23 & 0.19 & 0.01 & 96.63 & 3.03 & 0.26 & 0.08 & 0.06 & -0.20 & 0.08 & 0.06 \\
\hline Sind & 64,805 & 3.17 & 3.85 & 0.68 & 99.50 & 0.49 & 0.00 & 0.00 & 98.38 & 1.61 & 0.01 & 0.00 & -1.12 & 1.12 & 0.00 & 0.00 \\
\hline Pakistan - All & 273,250 & 3.92 & 4.62 & 0.70 & 94.68 & 4.09 & 0.98 & 0.25 & 93.53 & 4.60 & 1.38 & 0.49 & -1.15 & 0.51 & 0.40 & 0.24 \\
\hline
\end{tabular}


Asia Pacific Region : Tree Cover on Agricultural Land (2010)

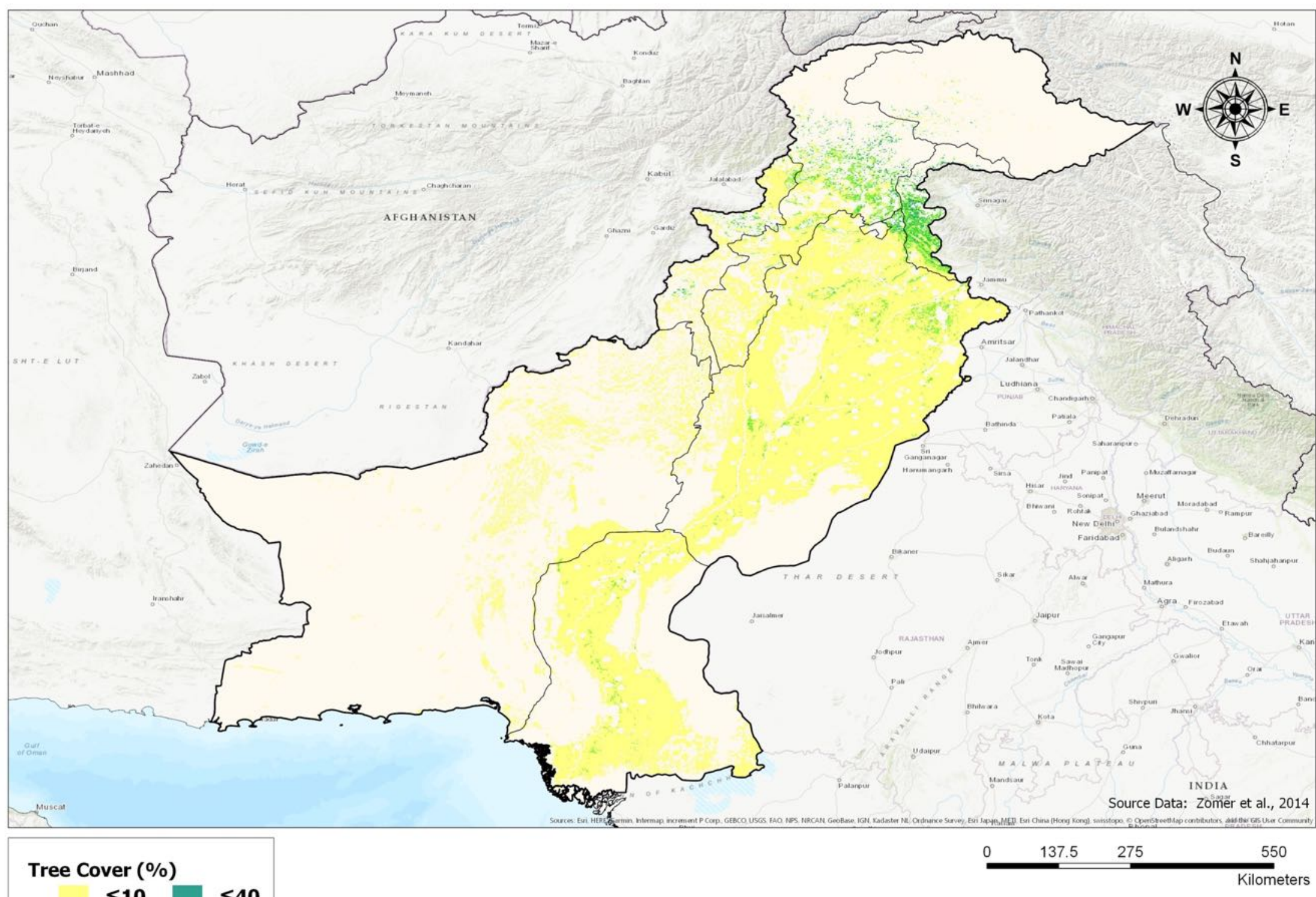

Pakistan 
Asia Pacific Region : Change in Tree Cover on Agricultural Land (2000 - 2010)

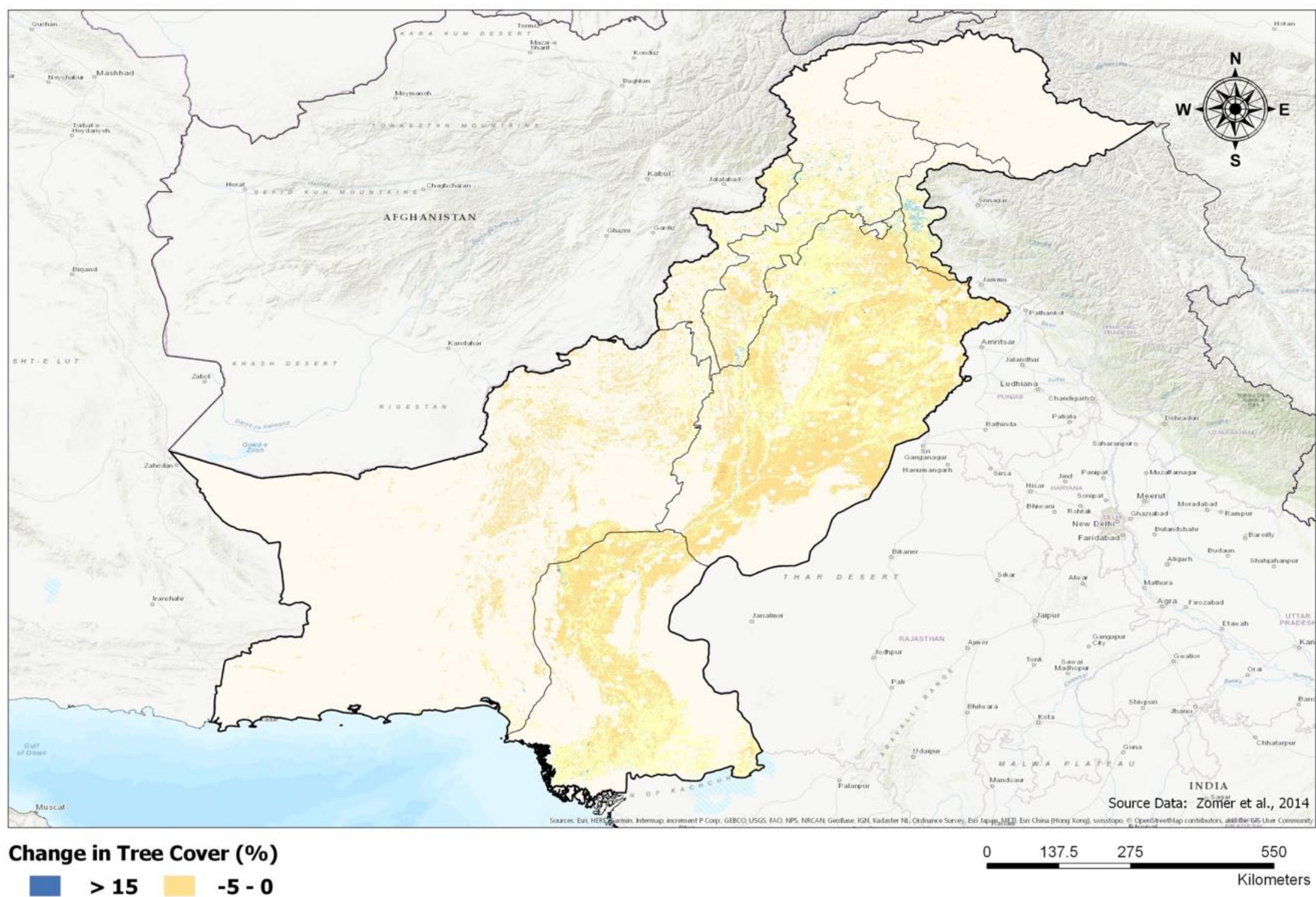

$\begin{aligned} 10-15 & -10--5 \\ 0 & -15--10\end{aligned}$

$0-5 \square \leq-15$

Pakistan 
Average Percent Tree Cover by Province, and Areal Extent of Tree Cover Classes $\left(\mathrm{km}^{2}\right)$

\begin{tabular}{|c|c|c|c|c|c|c|c|c|c|c|c|c|c|c|c|c|}
\hline \multicolumn{2}{|c|}{ Papua New Guinea } & \multicolumn{3}{|c|}{ Average Tree Cover by Province } & \multicolumn{12}{|c|}{ Areal Extent of Tree Cover Class $\left(\mathrm{km}^{2}\right)$} \\
\hline \multirow{3}{*}{ Province } & \multirow{3}{*}{ Area_km } & \multirow{2}{*}{\multicolumn{3}{|c|}{$\begin{array}{l}\text { Mean Tree Cover Percent by Province } \\
(\%)\end{array}$}} & \multirow{2}{*}{\multicolumn{4}{|c|}{ Year 2000}} & \multicolumn{4}{|c|}{ Year 2010} & \multirow{2}{*}{\multicolumn{4}{|c|}{$\begin{array}{l}\text { Change (2000 to 2010) } \\
\end{array}$}} \\
\hline & & & & & & & & & & a by Tree $\mathrm{C}$ & er Class $(k$ & & & & & \\
\hline & & 2000 & 2010 & Change & $<10 \%$ & $10-20 \%$ & $20-30 \%$ & $>30 \%$ & $<10 \%$ & $10-20 \%$ & $20-30 \%$ & $>30 \%$ & $<10 \%$ & $10-20 \%$ & $20-30 \%$ & $>30 \%$ \\
\hline Bougainville & 166 & 62.45 & 71.30 & 8.84 & 0 & 0 & $\overline{0}$ & 166 & 0 & 0 & 0 & 166 & 0 & 0 & 0 & 0 \\
\hline Central & 6,697 & 49.63 & 52.70 & 3.07 & 59 & 663 & 890 & 5,085 & 6 & 339 & 927 & 5,425 & -53 & -324 & 37 & 340 \\
\hline Chimbu & 993 & 39.25 & 44.55 & 5.30 & 16 & 183 & 226 & 568 & 0 & 91 & 166 & 736 & -16 & -92 & -60 & 168 \\
\hline East New Britain & 2,055 & 61.60 & 67.74 & 6.13 & 0 & 6 & 20 & 2,029 & 0 & 3 & 6 & 2,046 & 0 & -3 & -14 & 17 \\
\hline East Sepik & 14,603 & 44.26 & 46.62 & 2.35 & 40 & 1,972 & 2,283 & 10,308 & 170 & 1,797 & 2,071 & 10,565 & 130 & -175 & -212 & 257 \\
\hline Eastern Highlands & 2,362 & 28.47 & 32.54 & 4.07 & 58 & 868 & 622 & 814 & 2 & 581 & 726 & 1,053 & -56 & -287 & 104 & 239 \\
\hline Enga & 932 & 40.57 & 44.09 & 3.52 & 10 & 72 & 215 & 635 & 2 & 43 & 161 & 726 & -8 & -29 & -54 & 91 \\
\hline Gulf & 4,412 & 67.05 & 62.93 & -4.11 & 1 & 30 & 121 & 4,260 & 0 & 38 & 181 & 4,193 & -1 & 8 & 60 & -67 \\
\hline Hela & 1,114 & 47.61 & 52.38 & 4.77 & 17 & 51 & 144 & 902 & 1 & 50 & 54 & 1,009 & -16 & -1 & -90 & 107 \\
\hline Jiwaka & 1,058 & 40.03 & 45.37 & 5.34 & 1 & 51 & 247 & 759 & 1 & 17 & 150 & 890 & 0 & -34 & -97 & 131 \\
\hline Madang & 5,039 & 53.32 & 57.63 & 4.31 & 54 & 282 & 325 & 4,378 & 28 & 205 & 335 & 4,471 & -26 & -77 & 10 & 93 \\
\hline Manus & 304 & 62.03 & 67.93 & 5.89 & 0 & 0 & 1 & 303 & 0 & 0 & 0 & 304 & 0 & 0 & -1 & 1 \\
\hline Milne Bay & 2,657 & 50.74 & 51.65 & 0.91 & 84 & 344 & 263 & 1,966 & 24 & 302 & 299 & 2,032 & -60 & -42 & 36 & 66 \\
\hline Morobe & 8,380 & 42.16 & 46.07 & 3.92 & 379 & 1,625 & 1,006 & 5,370 & 188 & 1,364 & 1,216 & 5,612 & -191 & -261 & 210 & 242 \\
\hline National Capital District & 3 & 32.00 & 34.33 & 2.33 & 0 & 2 & 0 & 1 & 0 & 0 & 2 & 1 & 0 & -2 & 2 & 0 \\
\hline New Ireland & 887 & 63.29 & 67.65 & 4.36 & 1 & 1 & 17 & 868 & 2 & 0 & 5 & 880 & 1 & -1 & -12 & 12 \\
\hline Oro & 5,058 & 56.89 & 58.96 & 2.08 & 1 & 154 & 339 & 4,564 & 19 & 132 & 332 & 4,575 & 18 & -22 & -7 & 11 \\
\hline Sandaun & 8,632 & 49.34 & 52.23 & 2.89 & 33 & 683 & 837 & 7,079 & 154 & 521 & 652 & 7,305 & 121 & -162 & -185 & 226 \\
\hline Southern Highlands & 1,919 & 52.18 & 55.19 & 3.01 & 4 & 109 & 309 & 1,497 & 8 & 27 & 237 & 1,647 & 4 & -82 & -72 & 150 \\
\hline West New Britain & 3,025 & 65.21 & 69.92 & 4.71 & 3 & 11 & 26 & 2,985 & 0 & 4 & 30 & 2,991 & -3 & -7 & 4 & 6 \\
\hline Western & 31,644 & 50.70 & 52.22 & 1.52 & 66 & 2,065 & 4,269 & 25,244 & 94 & 1,906 & 3,527 & 26,117 & 28 & -159 & -742 & 873 \\
\hline Western Highlands & 1,130 & 35.31 & 39.75 & 4.44 & 2 & 146 & 314 & 668 & 0 & 64 & 242 & 824 & -2 & -82 & -72 & 156 \\
\hline Papua New Guinea - All & 103,070 & 49.85 & 52.29 & 2.44 & 829 & 9,318 & 12,474 & 80,450 & 699 & 7,484 & 11,319 & 83,569 & -130 & $-1,834$ & $-1,155$ & 3,119 \\
\hline
\end{tabular}


Average Percent Tree Cover by Province, and Areal Extent of Tree Cover Classes as a Percent of the Total Agricultural Area (\%)

\begin{tabular}{|c|c|c|c|c|c|c|c|c|c|c|c|c|c|c|c|c|}
\hline \multicolumn{2}{|c|}{ Papua New Guinea } & \multirow{3}{*}{\multicolumn{3}{|c|}{$\begin{array}{c}\text { Average Tree Cover by Province } \\
\text { Percent Tree Cover } \\
(\%)\end{array}$}} & \multicolumn{12}{|c|}{ Areal Extent of Tree Cover Class as a Percent of Total Agricultural Area (\%) } \\
\hline \multirow{3}{*}{ Province } & \multirow{3}{*}{ Area_km } & & & & \multirow{2}{*}{\multicolumn{4}{|c|}{$\begin{array}{c}\text { Year } 2000 \\
\text { Percent of Total Agrciultural Area (\%) }\end{array}$}} & & Yea & 2010 & & & Change $(2$ & 00 to 2010) & \\
\hline & & & & & & & & & \multicolumn{4}{|c|}{ Percent of Total Agrciultural Area (\%) } & \multicolumn{4}{|c|}{ Percent of Total Agrciultural Area (\%) } \\
\hline & & 2000 & 2010 & Change & $<10 \%$ & $10-20 \%$ & $20-30 \%$ & $>30 \%$ & $<10 \%$ & $10-20 \%$ & $20-30 \%$ & $>30 \%$ & $<10 \%$ & $10-20 \%$ & $20-30 \%$ & $>30 \%$ \\
\hline Bougainville & 166 & 62.45 & 71.30 & 8.84 & 0.00 & 0.00 & 0.00 & 100.00 & 0.00 & 0.00 & 0.00 & 100.00 & 0.00 & 0.00 & 0.00 & 0.00 \\
\hline Central & 6,697 & 49.63 & 52.70 & 3.07 & 0.88 & 9.90 & 13.29 & 75.93 & 0.09 & 5.06 & 13.84 & 81.01 & -0.79 & -4.84 & 0.55 & 5.08 \\
\hline Chimbu & 993 & 39.25 & 44.55 & 5.30 & 1.61 & 18.43 & 22.76 & 57.20 & 0.00 & 9.16 & 16.72 & 74.12 & -1.61 & -9.26 & -6.04 & 16.92 \\
\hline East New Britain & 2,055 & 61.60 & 67.74 & 6.13 & 0.00 & 0.29 & 0.97 & 98.74 & 0.00 & 0.15 & 0.29 & 99.56 & 0.00 & -0.15 & -0.68 & 0.83 \\
\hline East Sepik & 14,603 & 44.26 & 46.62 & 2.35 & 0.27 & 13.50 & 15.63 & 70.59 & 1.16 & 12.31 & 14.18 & 72.35 & 0.89 & -1.20 & -1.45 & 1.76 \\
\hline Eastern Highlands & 2,362 & 28.47 & 32.54 & 4.07 & 2.46 & 36.75 & 26.33 & 34.46 & 0.08 & 24.60 & 30.74 & 44.58 & -2.37 & -12.15 & 4.40 & 10.12 \\
\hline Enga & 932 & 40.57 & 44.09 & 3.52 & 1.07 & 7.73 & 23.07 & 68.13 & 0.21 & 4.61 & 17.27 & 77.90 & -0.86 & -3.11 & -5.79 & 9.76 \\
\hline Gulf & 4,412 & 67.05 & 62.93 & -4.11 & 0.02 & 0.68 & 2.74 & 96.56 & 0.00 & 0.86 & 4.10 & 95.04 & -0.02 & 0.18 & 1.36 & -1.52 \\
\hline Hela & 1,114 & 47.61 & 52.38 & 4.77 & 1.53 & 4.58 & 12.93 & 80.97 & 0.09 & 4.49 & 4.85 & 90.58 & -1.44 & -0.09 & -8.08 & 9.61 \\
\hline Jiwaka & 1,058 & 40.03 & 45.37 & 5.34 & 0.09 & 4.82 & 23.35 & 71.74 & 0.09 & 1.61 & 14.18 & 84.12 & 0.00 & -3.21 & -9.17 & 12.38 \\
\hline Madang & 5,039 & 53.32 & 57.63 & 4.31 & 1.07 & 5.60 & 6.45 & 86.88 & 0.56 & 4.07 & 6.65 & 88.73 & -0.52 & -1.53 & 0.20 & 1.85 \\
\hline Manus & 304 & 62.03 & 67.93 & 5.89 & 0.00 & 0.00 & 0.33 & 99.67 & 0.00 & 0.00 & 0.00 & 100.00 & 0.00 & 0.00 & -0.33 & 0.33 \\
\hline Milne Bay & 2,657 & 50.74 & 51.65 & 0.91 & 3.16 & 12.95 & 9.90 & 73.99 & 0.90 & 11.37 & 11.25 & 76.48 & -2.26 & -1.58 & 1.35 & 2.48 \\
\hline Morobe & 8,380 & 42.16 & 46.07 & 3.92 & 4.52 & 19.39 & 12.00 & 64.08 & 2.24 & 16.28 & 14.51 & 66.97 & -2.28 & -3.11 & 2.51 & 2.89 \\
\hline National Capital District & 3 & 32.00 & 34.33 & 2.33 & 0.00 & 66.67 & 0.00 & 33.33 & 0.00 & 0.00 & 66.67 & 33.33 & 0.00 & -66.67 & 66.67 & 0.00 \\
\hline New Ireland & 887 & 63.29 & 67.65 & 4.36 & 0.11 & 0.11 & 1.92 & 97.86 & 0.23 & 0.00 & 0.56 & 99.21 & 0.11 & -0.11 & -1.35 & 1.35 \\
\hline Oro & 5,058 & 56.89 & 58.96 & 2.08 & 0.02 & 3.04 & 6.70 & 90.23 & 0.38 & 2.61 & 6.56 & 90.45 & 0.36 & -0.43 & -0.14 & 0.22 \\
\hline Sandaun & 8,632 & 49.34 & 52.23 & 2.89 & 0.38 & 7.91 & 9.70 & 82.01 & 1.78 & 6.04 & 7.55 & 84.63 & 1.40 & -1.88 & -2.14 & 2.62 \\
\hline Southern Highlands & 1,919 & 52.18 & 55.19 & 3.01 & 0.21 & 5.68 & 16.10 & 78.01 & 0.42 & 1.41 & 12.35 & 85.83 & 0.21 & -4.27 & -3.75 & 7.82 \\
\hline West New Britain & 3,025 & 65.21 & 69.92 & 4.71 & 0.10 & 0.36 & 0.86 & 98.68 & 0.00 & 0.13 & 0.99 & 98.88 & -0.10 & -0.23 & 0.13 & 0.20 \\
\hline Western & 31,644 & 50.70 & 52.22 & 1.52 & 0.21 & 6.53 & 13.49 & 79.78 & 0.30 & 6.02 & 11.15 & 82.53 & 0.09 & -0.50 & -2.34 & 2.76 \\
\hline Western Highlands & 1,130 & 35.31 & 39.75 & 4.44 & 0.18 & 12.92 & 27.79 & 59.12 & 0.00 & 5.66 & 21.42 & 72.92 & -0.18 & -7.26 & -6.37 & 13.81 \\
\hline Papua New Guinea - All & 103,070 & 49.85 & 52.29 & 2.44 & 0.80 & 9.04 & 12.10 & 78.05 & 0.68 & 7.26 & 10.98 & 81.08 & -0.13 & -1.78 & -1.12 & 3.03 \\
\hline
\end{tabular}


Asia Pacific Region : Tree Cover on Agricultural Land (2010)

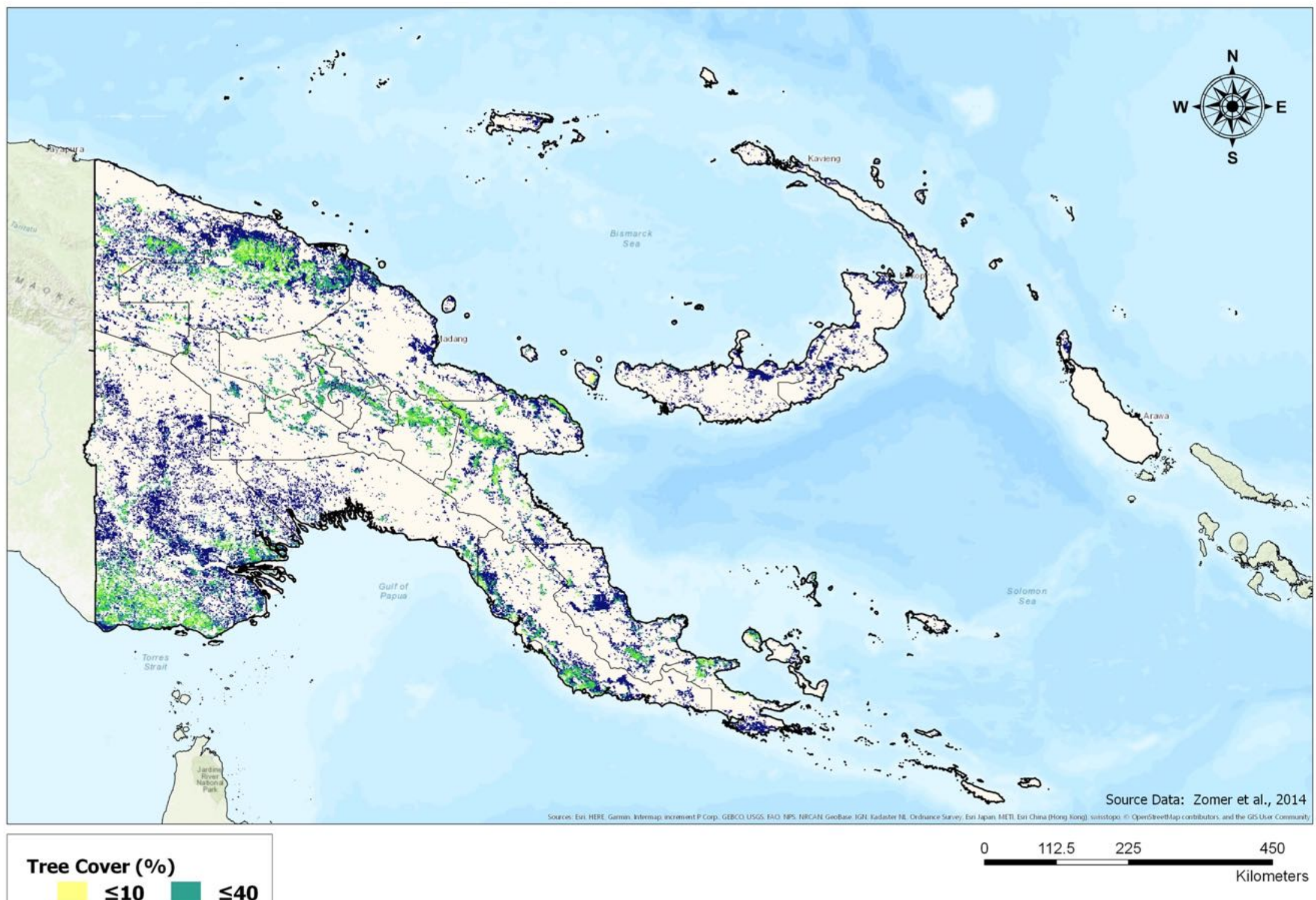

Papua New Guinea 
Asia Pacific Region : Change in Tree Cover on Agricultural Land (2000 - 2010)

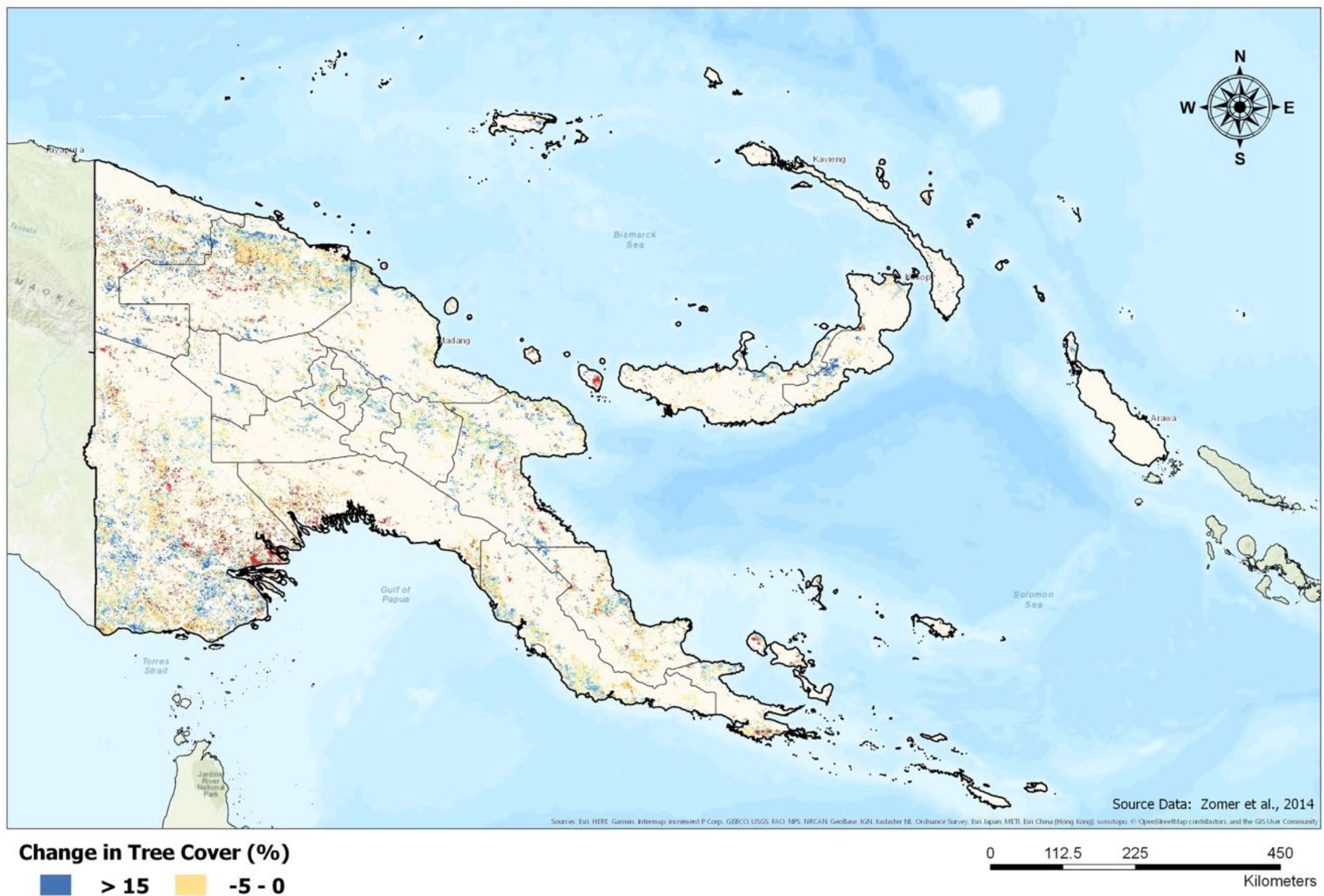
$\begin{array}{r}>15 \\ 10-15\end{array}-10-0$
$\begin{aligned} 10-15 & -10--5 \\ 5-10 & -15--10\end{aligned}$
$0-5 \square \leq-15$

\section{Papua New Guinea}




\section{Appendix 20: Philippines}

Average Percent Tree Cover by Province, and Areal Extent of Tree Cover Classes (km2)

\begin{tabular}{|c|c|c|c|c|c|c|c|c|c|c|c|c|c|c|c|c|}
\hline \multicolumn{2}{|c|}{ Philippines } & \multirow{3}{*}{\multicolumn{3}{|c|}{\begin{tabular}{|c|} 
Average Tree Cover by Province \\
Mean Tree Cover Percent by Province \\
(\%)
\end{tabular}}} & \multicolumn{12}{|c|}{ Areal Extent of Tree Cover Class $\left(\mathrm{km}^{2}\right)$} \\
\hline \multirow{3}{*}{ Province } & \multirow{3}{*}{ Area_km } & & & & \multirow{2}{*}{\multicolumn{4}{|c|}{$\begin{array}{c}\text { Year } 2000 \\
\text { Area by Tree Cover Class }\left(\mathrm{km}^{2}\right)\end{array}$}} & \multirow{2}{*}{\multicolumn{4}{|c|}{$\begin{array}{c}\text { Year } 2010 \\
\text { Area by Tree Cover Class }\left(\mathrm{km}^{2}\right)\end{array}$}} & \multirow{2}{*}{\multicolumn{4}{|c|}{$\begin{array}{c}\text { Change (2000 to } 2010) \\
\text { Area by Tree Cover Class }\left(\mathrm{km}^{2}\right)\end{array}$}} \\
\hline & & & & & & & & & & & & & & & & \\
\hline & & 2000 & 2010 & Change & $<10 \%$ & $10-20 \%$ & $20-30 \%$ & $>30 \%$ & $<10 \%$ & $10-20 \%$ & $20-30 \%$ & $>30 \%$ & $<10 \%$ & $10-20 \%$ & $20-30 \%$ & $>30 \%$ \\
\hline Abra & 2,436 & 33.34 & 36.97 & 3.63 & 138 & 304 & 584 & 1,410 & 116 & 151 & 414 & 1,755 & -22 & -153 & -170 & 345 \\
\hline Agusan del Norte & 1,470 & 40.09 & 47.72 & 7.63 & 0 & 103 & 371 & 996 & 1 & 43 & 194 & 1,232 & 1 & -60 & -177 & 236 \\
\hline Agusan del Sur & 4,315 & 51.36 & 56.93 & 5.57 & 0 & 254 & 423 & 3,638 & 0 & 172 & 372 & 3,771 & 0 & -82 & -51 & 133 \\
\hline Aklan & 1,128 & 32.24 & 33.94 & 1.70 & 6 & 180 & 361 & 581 & 13 & 213 & 276 & 626 & 7 & 33 & -85 & 45 \\
\hline Albay & 1,774 & 38.38 & 35.48 & -2.90 & 7 & 222 & 403 & 1,142 & 20 & 327 & 426 & 1,001 & 13 & 105 & 23 & -141 \\
\hline Antique & 2,111 & 26.77 & 29.55 & 2.78 & 45 & 707 & 697 & 662 & 54 & 436 & 720 & 901 & 9 & -271 & 23 & 239 \\
\hline Apayao & 928 & 42.11 & 37.47 & -4.63 & 26 & 98 & 143 & 661 & 29 & 146 & 142 & 611 & 3 & 48 & -1 & -50 \\
\hline Aurora & 1,023 & 53.59 & 51.88 & -1.71 & 0 & 49 & 111 & 863 & 0 & 42 & 127 & 854 & 0 & -7 & 16 & -9 \\
\hline Basilan & 803 & 54.27 & 53.58 & -0.69 & 0 & 1 & 24 & 778 & 0 & 22 & 56 & 725 & 0 & 21 & 32 & -53 \\
\hline Bataan & 844 & 31.01 & 36.26 & 5.25 & 25 & 207 & 220 & 392 & 2 & 116 & 199 & 527 & -23 & -91 & -21 & 135 \\
\hline Batangas & 2,345 & 33.87 & 38.88 & 5.01 & 13 & 375 & 651 & 1,306 & 10 & 222 & 526 & 1,587 & -3 & -153 & -125 & 281 \\
\hline Benguet & 1,611 & 34.44 & 40.41 & 5.96 & 8 & 200 & 489 & 914 & 3 & 45 & 291 & 1,272 & -5 & -155 & -198 & 358 \\
\hline Biliran & 261 & 40.58 & 40.69 & 0.11 & 2 & 31 & 40 & 188 & 0 & 32 & 37 & 192 & -2 & 1 & -3 & 4 \\
\hline Bohol & 3,380 & 26.32 & 28.60 & 2.28 & 149 & 1,316 & 801 & 1,114 & 163 & 1,197 & 696 & 1,324 & 14 & -119 & -105 & 210 \\
\hline Bukidnon & 6,110 & 31.02 & 33.59 & 2.57 & 3 & 845 & 2,707 & 2,555 & 4 & 484 & 2,396 & 3,226 & 1 & -361 & -311 & 671 \\
\hline Bulacan & 1,120 & 30.31 & 32.02 & 1.71 & 83 & 398 & 137 & 502 & 8 & 340 & 232 & 540 & -75 & -58 & 95 & 38 \\
\hline Cagayan & 4,729 & 28.47 & 25.39 & -3.08 & 232 & 1,788 & 983 & 1,726 & 372 & 2,005 & 942 & 1,410 & 140 & 217 & -41 & -316 \\
\hline Camarines Norte & 1,424 & 43.65 & 46.09 & 2.44 & 8 & 87 & 180 & 1,149 & 8 & 79 & 143 & 1,194 & 0 & -8 & -37 & 45 \\
\hline Camarines Sur & 3,917 & 39.71 & 40.27 & 0.56 & 2 & 605 & 713 & 2,597 & 13 & 662 & 658 & 2,584 & 11 & 57 & -55 & -13 \\
\hline Camiguin & 16 & 46.88 & 59.63 & 12.75 & 0 & 0 & 2 & 14 & 0 & 0 & 0 & 16 & 0 & 0 & -2 & 2 \\
\hline Capiz & 2,280 & 22.31 & 21.71 & -0.60 & 0 & 1,143 & 873 & 264 & 149 & 1,099 & 623 & 409 & 149 & -44 & -250 & 145 \\
\hline Catanduanes & 763 & 51.55 & 55.46 & 3.91 & 0 & 20 & 51 & 692 & 0 & 19 & 31 & 713 & 0 & -1 & -20 & 21 \\
\hline Cavite & 757 & 27.25 & 35.92 & 8.67 & 16 & 213 & 277 & 251 & 13 & 138 & 145 & 461 & -3 & -75 & -132 & 210 \\
\hline Cebu & 4,019 & 23.54 & 25.69 & 2.15 & 214 & 1,551 & 1,382 & 872 & 114 & 1,300 & 1,512 & 1,093 & -100 & -251 & 130 & 221 \\
\hline Compostela Valley & 2,852 & 45.81 & 49.18 & 3.38 & 0 & 83 & 409 & 2,360 & 0 & 35 & 246 & 2,571 & 0 & -48 & -163 & 211 \\
\hline Davao del Norte & 2,742 & 42.00 & 43.65 & 1.65 & 1 & 94 & 632 & 2,015 & 0 & 135 & 501 & 2,106 & -1 & 41 & -131 & 91 \\
\hline Davao del Sur & 4,412 & 35.49 & 36.95 & 1.46 & 0 & 394 & 1,366 & 2,652 & 30 & 499 & 942 & 2,941 & 30 & 105 & -424 & 289 \\
\hline Davao Oriental & 2,597 & 50.76 & 55.21 & 4.45 & 3 & 50 & 235 & 2,309 & 0 & 12 & 126 & 2,459 & -3 & -38 & -109 & 150 \\
\hline Dinagat Islands & 174 & 28.26 & 33.10 & 4.84 & 1 & 58 & 49 & 66 & 0 & 36 & 49 & 89 & -1 & -22 & 0 & 23 \\
\hline Eastern Samar & 1,814 & 50.77 & 50.28 & -0.49 & 0 & 29 & 91 & 1,694 & 0 & 16 & 129 & 1,669 & 0 & -13 & 38 & -25 \\
\hline Guimaras & 506 & 34.76 & 32.15 & -2.61 & 5 & 78 & 154 & 269 & 3 & 74 & 191 & 238 & -2 & -4 & 37 & -31 \\
\hline Ifugao & 1,592 & 27.89 & 31.18 & 3.29 & 18 & 639 & 421 & 514 & 10 & 481 & 427 & 674 & -8 & -158 & 6 & 160 \\
\hline llocos Norte & 2,234 & 29.89 & 34.70 & 4.81 & 136 & 576 & 525 & 997 & 66 & 388 & 459 & 1,321 & -70 & -188 & -66 & 324 \\
\hline Ilocos Sur & 2,089 & 31.63 & 37.30 & 5.67 & 190 & 522 & 358 & 1,019 & 46 & 394 & 339 & 1,310 & -144 & -128 & -19 & 291 \\
\hline Iloilo & 4,099 & 24.21 & 22.28 & -1.93 & 60 & 1,719 & 1,410 & 910 & 395 & 1,846 & 1,031 & 827 & 335 & 127 & -379 & -83 \\
\hline Isabela & 6,034 & 22.30 & 17.49 & -4.81 & 372 & 3,433 & 1,186 & 1,043 & 1,115 & 3,792 & 557 & 570 & 743 & 359 & -629 & -473 \\
\hline Kalinga & 1,568 & 33.43 & 33.11 & -0.32 & 23 & 513 & 317 & 715 & 76 & 459 & 287 & 746 & 53 & -54 & -30 & 31 \\
\hline La Union & 1,215 & 31.56 & 37.78 & 6.22 & 49 & 225 & 320 & 621 & 6 & 170 & 161 & 878 & -43 & -55 & -159 & 257 \\
\hline Laguna & 903 & 39.70 & 41.46 & 1.77 & 9 & 154 & 137 & 603 & 3 & 136 & 165 & 599 & -6 & -18 & 28 & -4 \\
\hline Lanao del Norte & 1,879 & 41.31 & 44.25 & 2.95 & 2 & 138 & 385 & 1,354 & 0 & 109 & 329 & 1,441 & -2 & -29 & -56 & 87 \\
\hline Lanao del Sur & 1,512 & 42.24 & 42.30 & 0.05 & 1 & 143 & 323 & 1,045 & 0 & 91 & 380 & 1,041 & -1 & -52 & 57 & -4 \\
\hline Leyte & 3,711 & 35.54 & 37.31 & 1.76 & 53 & 706 & 724 & 2,228 & 24 & 544 & 831 & 2,312 & -29 & -162 & 107 & 84 \\
\hline Maguindanao & 3,931 & 30.61 & 31.55 & 0.94 & 10 & 868 & 1,531 & 1,522 & 4 & 664 & 1,629 & 1,634 & -6 & -204 & 98 & 112 \\
\hline Marinduque & 549 & 43.76 & 47.38 & 3.62 & 7 & 20 & 66 & 456 & 5 & 13 & 60 & 471 & -2 & -7 & -6 & 15 \\
\hline Masbate & 3,371 & 27.55 & 28.36 & 0.81 & 132 & 1,158 & 926 & 1,155 & 113 & 1,177 & 840 & 1,241 & -19 & 19 & -86 & 86 \\
\hline Misamis Occidental & 842 & 34.99 & 38.61 & 3.62 & 0 & 86 & 195 & 561 & 5 & 34 & 152 & 651 & 5 & -52 & -43 & 90 \\
\hline
\end{tabular}




\begin{tabular}{|c|c|c|c|c|c|c|c|c|c|c|c|c|c|c|c|c|}
\hline \multicolumn{2}{|c|}{ Philippines } & \multicolumn{3}{|c|}{ Average Tree Cover by Province } & \multicolumn{12}{|c|}{ Areal Extent of Tree Cover Class $\left(\mathrm{km}^{2}\right)$} \\
\hline \multirow{3}{*}{ Province } & \multirow{3}{*}{ Area_km } & \multirow{2}{*}{\multicolumn{3}{|c|}{$\begin{array}{c}\text { Mean Tree Cover Percent by Province } \\
(\%)\end{array}$}} & \multicolumn{4}{|c|}{ Year 2000} & \multirow{2}{*}{\multicolumn{4}{|c|}{$\begin{array}{c}\text { Year } 2010 \\
\text { Area by Tree Cover Class }\left(\mathbf{k m}^{2}\right)\end{array}$}} & \multirow{2}{*}{\multicolumn{4}{|c|}{$\begin{array}{c}\text { Change }(2000 \text { to } 2010) \\
\text { Area by Tree Cover Class }\left(\mathrm{km}^{2}\right)\end{array}$}} \\
\hline & & & & & & ea by Tree & ver Class & & & & & & & & & \\
\hline & & 2000 & 2010 & Change & $<10 \%$ & $10-20 \%$ & $20-30 \%$ & $>30 \%$ & $<10 \%$ & $10-20 \%$ & $20-30 \%$ & $>30 \%$ & $<10 \%$ & $10-20 \%$ & $20-30 \%$ & $>30 \%$ \\
\hline Mountain Province & 1,097 & 37.29 & 38.70 & 1.41 & 8 & 202 & 271 & 616 & 2 & 128 & 218 & 749 & -6 & -74 & -53 & 133 \\
\hline Negros Occidental & 6,677 & 21.92 & 22.81 & 0.89 & 184 & 3,561 & 1,952 & 980 & 210 & 3,384 & 1,920 & 1,163 & 26 & -177 & -32 & 183 \\
\hline Negros Oriental & 4,475 & 25.51 & 24.90 & -0.60 & 63 & 1,592 & 1,557 & 1,263 & 179 & 1,574 & 1,500 & 1,222 & 116 & -18 & -57 & -41 \\
\hline North Cotabato & 5,297 & 30.78 & 31.60 & 0.82 & 2 & 855 & 2,250 & 2,190 & 3 & 799 & 1,929 & 2,566 & 1 & -56 & -321 & 376 \\
\hline Northern Samar & 1,797 & 45.91 & 52.60 & 6.70 & 0 & 103 & 187 & 1,507 & 0 & 42 & 153 & 1,602 & 0 & -61 & -34 & 95 \\
\hline Nueva Ecija & 4,571 & 19.65 & 23.96 & 4.31 & 1,510 & 1,688 & 516 & 857 & 272 & 2,415 & 808 & 1,076 & $-1,238$ & 727 & 292 & 219 \\
\hline Nueva Vizcaya & 2,536 & 28.74 & 30.03 & 1.30 & 65 & 854 & 732 & 885 & 40 & 788 & 735 & 973 & -25 & -66 & 3 & 88 \\
\hline Occidental Mindoro & 4,679 & 33.41 & 34.55 & 1.14 & 304 & 1,094 & 796 & 2,485 & 273 & 1,010 & 808 & 2,588 & -31 & -84 & 12 & 103 \\
\hline Oriental Mindoro & 2,914 & 39.21 & 39.05 & -0.16 & 25 & 333 & 543 & 2,013 & 32 & 365 & 531 & 1,986 & 7 & 32 & -12 & -27 \\
\hline Palawan & 6,715 & 44.62 & 44.03 & -0.59 & 18 & 532 & 918 & 5,247 & 44 & 599 & 921 & 5,151 & 26 & 67 & 3 & -96 \\
\hline Pampanga & 1,315 & 19.98 & 24.17 & 4.18 & 296 & 585 & 214 & 220 & 63 & 655 & 309 & 288 & -233 & 70 & 95 & 68 \\
\hline Pangasinan & 4,645 & 19.94 & 24.77 & 4.84 & 1,080 & 1,894 & 795 & 876 & 219 & 2,048 & 1,053 & 1,325 & -861 & 154 & 258 & 449 \\
\hline Quezon & 4,804 & 47.33 & 48.90 & 1.58 & 2 & 206 & 570 & 4,026 & 12 & 167 & 491 & 4,134 & 10 & -39 & -79 & 108 \\
\hline Quirino & 1,172 & 34.88 & 27.92 & -6.97 & 0 & 187 & 391 & 594 & 8 & 448 & 299 & 417 & 8 & 261 & -92 & -177 \\
\hline Rizal & 712 & 35.29 & 40.63 & 5.34 & 18 & 100 & 168 & 426 & 0 & 41 & 135 & 536 & -18 & -59 & -33 & 110 \\
\hline Romblon & 679 & 40.88 & 39.05 & -1.83 & 0 & 53 & 147 & 479 & 1 & 69 & 148 & 461 & 1 & 16 & 1 & -18 \\
\hline Samar & 2,786 & 41.93 & 42.16 & 0.23 & 0 & 287 & 531 & 1,968 & 4 & 300 & 449 & 2,033 & 4 & 13 & -82 & 65 \\
\hline Sarangani & 2,398 & 32.89 & 36.18 & 3.29 & 30 & 471 & 744 & 1,153 & 3 & 277 & 710 & 1,408 & -27 & -194 & -34 & 255 \\
\hline Siquijor & 264 & 27.37 & 33.49 & 6.12 & 5 & 60 & 109 & 90 & 0 & 25 & 93 & 146 & -5 & -35 & -16 & 56 \\
\hline Sorsogon & 1,282 & 44.90 & 45.00 & 0.10 & 11 & 102 & 148 & 1,021 & 11 & 86 & 166 & 1,019 & 0 & -16 & 18 & -2 \\
\hline South Cotabato & 3,095 & 30.76 & 33.85 & 3.09 & 65 & 701 & 1,051 & 1,278 & 5 & 282 & 1,202 & 1,606 & -60 & -419 & 151 & 328 \\
\hline Southern Leyte & 951 & 45.88 & 51.68 & 5.81 & 0 & 38 & 124 & 789 & 0 & 20 & 75 & 856 & 0 & -18 & -49 & 67 \\
\hline Sultan Kudarat & 3,226 & 34.54 & 35.52 & 0.98 & 22 & 668 & 1,022 & 1,514 & 2 & 522 & 1,118 & 1,584 & -20 & -146 & 96 & 70 \\
\hline Sulu & 647 & 52.73 & 55.62 & 2.89 & 1 & 3 & 10 & 633 & 3 & 0 & 4 & 640 & 2 & -3 & -6 & 7 \\
\hline Surigao del Norte & 563 & 44.35 & 46.79 & 2.43 & 1 & 64 & 81 & 417 & 0 & 41 & 86 & 436 & -1 & -23 & 5 & 19 \\
\hline Surigao del Sur & 1,735 & 52.76 & 56.79 & 4.03 & 0 & 93 & 117 & 1,525 & 0 & 64 & 127 & 1,544 & 0 & -29 & 10 & 19 \\
\hline Tarlac & 2,536 & 22.14 & 28.22 & 6.07 & 699 & 860 & 296 & 681 & 93 & 1,090 & 521 & 832 & -606 & 230 & 225 & 151 \\
\hline Tawi-Tawi & 218 & 57.33 & 60.71 & 3.38 & 0 & 2 & 9 & 207 & 0 & 0 & 2 & 216 & 0 & -2 & -7 & 9 \\
\hline Zambales & 2,712 & 23.68 & 28.20 & 4.52 & 635 & 727 & 510 & 840 & 171 & 820 & 707 & 1,014 & -464 & 93 & 197 & 174 \\
\hline Zamboanga del Norte & 4,723 & 37.92 & 41.08 & 3.16 & 7 & 375 & 1,193 & 3,148 & 6 & 133 & 806 & 3,778 & -1 & -242 & -387 & 630 \\
\hline Zamboanga del Sur & 3,952 & 32.97 & 37.05 & 4.09 & 27 & 679 & 1,153 & 2,093 & 1 & 332 & 964 & 2,655 & -26 & -347 & -189 & 562 \\
\hline Zamboanga Sibugay & 2,086 & 38.98 & 41.42 & 2.43 & 16 & 180 & 379 & 1,511 & 2 & 141 & 316 & 1,627 & -14 & -39 & -63 & 116 \\
\hline Philippines - All & 188,841 & 33.79 & 35.58 & 1.80 & 7,148 & 41,771 & 44,258 & 95,665 & 4,655 & 39,200 & 40,563 & 104,424 & $-2,493$ & $-2,571$ & $-3,695$ & 8,759 \\
\hline
\end{tabular}




\begin{tabular}{|c|c|c|c|c|c|c|c|c|c|c|c|c|c|c|c|c|}
\hline \multicolumn{2}{|c|}{ Philippines } & \multirow{3}{*}{\multicolumn{3}{|c|}{$\begin{array}{l}\text { Average Tree Cover by Province } \\
\text { Percent Tree Cover } \\
(\%)\end{array}$}} & \multicolumn{12}{|c|}{ Areal Extent of Tree Cover Class as a Percent of Total Agricultural Area (\%) } \\
\hline \multirow{3}{*}{ Province } & \multirow{3}{*}{ Area_km } & & & & \multirow{2}{*}{\multicolumn{4}{|c|}{$\begin{array}{c}\text { Year 2000 } \\
\text { Percent of Total Agrciultural Area ( \%) }\end{array}$}} & \multirow{2}{*}{\multicolumn{4}{|c|}{$\begin{array}{c}\text { Year } 2010 \\
\text { Percent of Total Agrciultural Area (\%) } \\
\end{array}$}} & \multirow{2}{*}{\multicolumn{4}{|c|}{$\begin{array}{c}\text { Change (2000 to 2010) } \\
\text { Percent of Total Agrciultural Area (\%) }\end{array}$}} \\
\hline & & & & & & & & & & & & & & & & \\
\hline & & 2000 & 2010 & Change & $<10 \%$ & $10-20 \%$ & $20-30 \%$ & $>30 \%$ & $<10 \%$ & $10-20 \%$ & $20-30 \%$ & $>30 \%$ & $<10 \%$ & $10-20 \%$ & $20-30 \%$ & $>30 \%$ \\
\hline Abra & 2,436 & 33.34 & 36.97 & 3.63 & 5.67 & 12.48 & 23.97 & 57.88 & 4.76 & 6.20 & 17.00 & 72.04 & -0.90 & -6.28 & -6.98 & 14.16 \\
\hline Agusan del Norte & 1,470 & 40.09 & 47.72 & 7.63 & 0.00 & 7.01 & 25.24 & 67.76 & 0.07 & 2.93 & 13.20 & 83.81 & 0.07 & -4.08 & -12.04 & 16.05 \\
\hline Agusan del Sur & 4,315 & 51.36 & 56.93 & 5.57 & 0.00 & 5.89 & 9.80 & 84.31 & 0.00 & 3.99 & 8.62 & 87.39 & 0.00 & -1.90 & -1.18 & 3.08 \\
\hline Aklan & 1,128 & 32.24 & 33.94 & 1.70 & 0.53 & 15.96 & 32.00 & 51.51 & 1.15 & 18.88 & 24.47 & 55.50 & 0.62 & 2.93 & -7.54 & 3.99 \\
\hline Albay & 1,774 & 38.38 & 35.48 & -2.90 & 0.39 & 12.51 & 22.72 & 64.37 & 1.13 & 18.43 & 24.01 & 56.43 & 0.73 & 5.92 & 1.30 & -7.95 \\
\hline Antique & 2,111 & 26.77 & 29.55 & 2.78 & 2.13 & 33.49 & 33.02 & 31.36 & 2.56 & 20.65 & 34.11 & 42.68 & 0.43 & -12.84 & 1.09 & 11.32 \\
\hline Apayao & 928 & 42.11 & 37.47 & -4.63 & 2.80 & 10.56 & 15.41 & 71.23 & 3.13 & 15.73 & 15.30 & 65.84 & 0.32 & 5.17 & -0.11 & -5.39 \\
\hline Aurora & 1,023 & 53.59 & 51.88 & -1.71 & 0.00 & 4.79 & 10.85 & 84.36 & 0.00 & 4.11 & 12.41 & 83.48 & 0.00 & -0.68 & 1.56 & -0.88 \\
\hline Basilan & 803 & 54.27 & 53.58 & -0.69 & 0.00 & 0.12 & 2.99 & 96.89 & 0.00 & 2.74 & 6.97 & 90.29 & 0.00 & 2.62 & 3.99 & -6.60 \\
\hline Bataan & 844 & 31.01 & 36.26 & 5.25 & 2.96 & 24.53 & 26.07 & 46.45 & 0.24 & 13.74 & 23.58 & 62.44 & -2.73 & -10.78 & -2.49 & 16.00 \\
\hline Batangas & 2,345 & 33.87 & 38.88 & 5.01 & 0.55 & 15.99 & 27.76 & 55.69 & 0.43 & 9.47 & 22.43 & 67.68 & -0.13 & -6.52 & -5.33 & 11.98 \\
\hline Benquet & 1,611 & 34.44 & 40.41 & 5.96 & 0.50 & 12.41 & 30.35 & 56.74 & 0.19 & 2.79 & 18.06 & 78.96 & -0.31 & -9.62 & -12.29 & 22.22 \\
\hline Biliran & 261 & 40.58 & 40.69 & 0.11 & 0.77 & 11.88 & 15.33 & 72.03 & 0.00 & 12.26 & 14.18 & 73.56 & -0.77 & 0.38 & -1.15 & 1.53 \\
\hline Bohol & 3,380 & 26.32 & 28.60 & 2.28 & 4.41 & 38.94 & 23.70 & 32.96 & 4.82 & 35.41 & 20.59 & 39.17 & 0.41 & -3.52 & -3.11 & 6.21 \\
\hline Bukidnon & 6,110 & 31.02 & 33.59 & 2.57 & 0.05 & 13.83 & 44.30 & 41.82 & 0.07 & 7.92 & 39.21 & 52.80 & 0.02 & -5.91 & -5.09 & 10.98 \\
\hline Bulacan & 1,120 & 30.31 & 32.02 & 1.71 & 7.41 & 35.54 & 12.23 & 44.82 & 0.71 & 30.36 & 20.71 & 48.21 & -6.70 & -5.18 & 8.48 & 3.39 \\
\hline Cagayan & 4,729 & 28.47 & 25.39 & -3.08 & 4.91 & 37.81 & 20.79 & 36.50 & 7.87 & 42.40 & 19.92 & 29.82 & 2.96 & 4.59 & -0.87 & -6.68 \\
\hline Camarines Norte & 1,424 & 43.65 & 46.09 & 2.44 & 0.56 & 6.11 & 12.64 & 80.69 & 0.56 & 5.55 & 10.04 & 83.85 & 0.00 & -0.56 & -2.60 & 3.16 \\
\hline Camarines Sur & 3,917 & 39.71 & 40.27 & 0.56 & 0.05 & 15.45 & 18.20 & 66.30 & 0.33 & 16.90 & 16.80 & 65.97 & 0.28 & 1.46 & -1.40 & -0.33 \\
\hline Camiguin & 16 & 46.88 & 59.63 & 12.75 & 0.00 & 0.00 & 12.50 & 87.50 & 0.00 & 0.00 & 0.00 & 100.00 & 0.00 & 0.00 & -12.50 & 12.50 \\
\hline Capiz & 2,280 & 22.31 & 21.71 & -0.60 & 0.00 & 50.13 & 38.29 & 11.58 & 6.54 & 48.20 & 27.32 & 17.94 & 6.54 & -1.93 & -10.96 & 6.36 \\
\hline Catanduanes & 763 & 51.55 & 55.46 & 3.91 & 0.00 & 2.62 & 6.68 & 90.70 & 0.00 & 2.49 & 4.06 & 93.45 & 0.00 & -0.13 & -2.62 & 2.75 \\
\hline Cavite & 757 & 27.25 & 35.92 & 8.67 & 2.11 & 28.14 & 36.59 & 33.16 & 1.72 & 18.23 & 19.15 & 60.90 & -0.40 & -9.91 & -17.44 & 27.74 \\
\hline Cebu & 4,019 & 23.54 & 25.69 & 2.15 & 5.32 & 38.59 & 34.39 & 21.70 & 2.84 & 32.35 & 37.62 & 27.20 & -2.49 & -6.25 & 3.23 & 5.50 \\
\hline Compostela Valley & 2,852 & 45.81 & 49.18 & 3.38 & 0.00 & 2.91 & 14.34 & 82.75 & 0.00 & 1.23 & 8.63 & 90.15 & 0.00 & -1.68 & -5.72 & 7.40 \\
\hline Davao del Norte & 2,742 & 42.00 & 43.65 & 1.65 & 0.04 & 3.43 & 23.05 & 73.49 & 0.00 & 4.92 & 18.27 & 76.81 & -0.04 & 1.50 & -4.78 & 3.32 \\
\hline Davao del Sur & 4,412 & 35.49 & 36.95 & 1.46 & 0.00 & 8.93 & 30.96 & 60.11 & 0.68 & 11.31 & 21.35 & 66.66 & 0.68 & 2.38 & -9.61 & 6.55 \\
\hline Davao Oriental & 2,597 & 50.76 & 55.21 & 4.45 & 0.12 & 1.93 & 9.05 & 88.91 & 0.00 & 0.46 & 4.85 & 94.69 & -0.12 & -1.46 & -4.20 & 5.78 \\
\hline Dinagat Islands & 174 & 28.26 & 33.10 & 4.84 & 0.57 & 33.33 & 28.16 & 37.93 & 0.00 & 20.69 & 28.16 & 51.15 & -0.57 & -12.64 & 0.00 & 13.22 \\
\hline Eastern Samar & 1,814 & 50.77 & 50.28 & -0.49 & 0.00 & 1.60 & 5.02 & 93.39 & 0.00 & 0.88 & 7.11 & 92.01 & 0.00 & -0.72 & 2.09 & -1.38 \\
\hline Guimaras & 506 & 34.76 & 32.15 & -2.61 & 0.99 & 15.42 & 30.44 & 53.16 & 0.59 & 14.62 & 37.75 & 47.04 & -0.40 & -0.79 & 7.31 & -6.13 \\
\hline Ifugao & 1,592 & 27.89 & 31.18 & 3.29 & 1.13 & 40.14 & 26.44 & 32.29 & 0.63 & 30.21 & 26.82 & 42.34 & -0.50 & -9.92 & 0.38 & 10.05 \\
\hline llocos Norte & 2,234 & 29.89 & 34.70 & 4.81 & 6.09 & 25.78 & 23.50 & 44.63 & 2.95 & 17.37 & 20.55 & 59.13 & -3.13 & -8.42 & -2.95 & 14.50 \\
\hline Ilocos Sur & 2,089 & 31.63 & 37.30 & 5.67 & 9.10 & 24.99 & 17.14 & 48.78 & 2.20 & 18.86 & 16.23 & 62.71 & -6.89 & -6.13 & -0.91 & 13.93 \\
\hline Iloilo & 4,099 & 24.21 & 22.28 & -1.93 & 1.46 & 41.94 & 34.40 & 22.20 & 9.64 & 45.04 & 25.15 & 20.18 & 8.17 & 3.10 & -9.25 & -2.02 \\
\hline Isabela & 6,034 & 22.30 & 17.49 & -4.81 & 6.17 & 56.89 & 19.66 & 17.29 & 18.48 & 62.84 & 9.23 & 9.45 & 12.31 & 5.95 & -10.42 & -7.84 \\
\hline Kalinga & 1,568 & 33.43 & 33.11 & -0.32 & 1.47 & 32.72 & 20.22 & 45.60 & 4.85 & 29.27 & 18.30 & 47.58 & 3.38 & -3.44 & -1.91 & 1.98 \\
\hline La Union & 1,215 & 31.56 & 37.78 & 6.22 & 4.03 & 18.52 & 26.34 & 51.11 & 0.49 & 13.99 & 13.25 & 72.26 & -3.54 & -4.53 & -13.09 & 21.15 \\
\hline Laguna & 903 & 39.70 & 41.46 & 1.77 & 1.00 & 17.05 & 15.17 & 66.78 & 0.33 & 15.06 & 18.27 & 66.33 & -0.66 & -1.99 & 3.10 & -0.44 \\
\hline Lanao del Norte & 1,879 & 41.31 & 44.25 & 2.95 & 0.11 & 7.34 & 20.49 & 72.06 & 0.00 & 5.80 & 17.51 & 76.69 & -0.11 & -1.54 & -2.98 & 4.63 \\
\hline Lanao del Sur & 1,512 & 42.24 & 42.30 & 0.05 & 0.07 & 9.46 & 21.36 & 69.11 & 0.00 & 6.02 & 25.13 & 68.85 & -0.07 & -3.44 & 3.77 & -0.26 \\
\hline Leyte & 3,711 & 35.54 & 37.31 & 1.76 & 1.43 & 19.02 & 19.51 & 60.04 & 0.65 & 14.66 & 22.39 & 62.30 & -0.78 & -4.37 & 2.88 & 2.26 \\
\hline Maguindanao & 3,931 & 30.61 & 31.55 & 0.94 & 0.25 & 22.08 & 38.95 & 38.72 & 0.10 & 16.89 & 41.44 & 41.57 & -0.15 & -5.19 & 2.49 & 2.85 \\
\hline Marinduque & 549 & 43.76 & 47.38 & 3.62 & 1.28 & 3.64 & 12.02 & 83.06 & 0.91 & 2.37 & 10.93 & 85.79 & -0.36 & -1.28 & -1.09 & 2.73 \\
\hline Masbate & 3,371 & 27.55 & 28.36 & 0.81 & 3.92 & 34.35 & 27.47 & 34.26 & 3.35 & 34.92 & 24.92 & 36.81 & -0.56 & 0.56 & -2.55 & 2.55 \\
\hline Misamis Occidental & 842 & 34.99 & 38.61 & 3.62 & 0.00 & 10.21 & 23.16 & 66.63 & 0.59 & 4.04 & 18.05 & 77.32 & 0.59 & -6.18 & -5.11 & 10.69 \\
\hline
\end{tabular}




\begin{tabular}{|c|c|c|c|c|c|c|c|c|c|c|c|c|c|c|c|c|}
\hline \multicolumn{2}{|c|}{ Philippines } & \multirow{3}{*}{\multicolumn{3}{|c|}{$\begin{array}{c}\text { Average Tree Cover by Province } \\
\text { Percent Tree Cover } \\
(\%)\end{array}$}} & \multicolumn{12}{|c|}{ Areal Extent of Tree Cover Class as a Percent of Total Agricultural Area (\%) } \\
\hline \multirow{3}{*}{ Province } & \multirow{3}{*}{ Area_km } & & & & \multirow{2}{*}{\multicolumn{4}{|c|}{$\begin{array}{c}\text { Year } 2000 \\
\text { Percent of Total Agrciultural Area (\%) }\end{array}$}} & \multirow{2}{*}{\multicolumn{4}{|c|}{$\begin{array}{c}\text { Year 2010 } \\
\text { Percent of Total Aarciultural Area (\%) }\end{array}$}} & \multirow{2}{*}{\multicolumn{4}{|c|}{ Change (2000 to 2010) }} \\
\hline & & & & & & & & & & & & & & & & \\
\hline & & 2000 & 2010 & & $<10 \%$ & $10-20 \%$ & $20-30 \%$ & $>30 \%$ & $<10 \%$ & $10-20 \%$ & $20-30 \%$ & $>30 \%$ & $<10 \%$ & $10-20 \%$ & $20-30 \%$ & $>30 \%$ \\
\hline Misamis Oriental & 1,392 & 33.27 & 38.72 & 5.45 & 0.36 & 17.17 & 28.09 & 54.38 & 0.22 & 10.06 & 19.40 & 70.33 & -0.14 & -7.11 & -8.69 & 15.95 \\
\hline Mountain Province & 1,097 & 37.29 & 38.70 & 1.41 & 0.73 & 18.41 & 24.70 & 56.15 & 0.18 & 11.67 & 19.87 & 68.28 & -0.55 & -6.75 & -4.83 & 12.12 \\
\hline Negros Occidental & 6,677 & 21.92 & 22.81 & 0.89 & 2.76 & 53.33 & 29.23 & 14.68 & 3.15 & 50.68 & 28.76 & 17.42 & 0.39 & -2.65 & -0.48 & 2.74 \\
\hline Negros Oriental & 4,475 & 25.51 & 24.90 & -0.60 & 1.41 & 35.58 & 34.79 & 28.22 & 4.00 & 35.17 & 33.52 & 27.31 & 2.59 & -0.40 & -1.27 & -0.92 \\
\hline North Cotabato & 5,297 & 30.78 & 31.60 & 0.82 & 0.04 & 16.14 & 42.48 & 41.34 & 0.06 & 15.08 & 36.42 & 48.44 & 0.02 & -1.06 & -6.06 & 7.10 \\
\hline Northern Samar & 1,797 & 45.91 & 52.60 & 6.70 & 0.00 & 5.73 & 10.41 & 83.86 & 0.00 & 2.34 & 8.51 & 89.15 & 0.00 & -3.39 & -1.89 & 5.29 \\
\hline Nueva Ecija & 4,571 & 19.65 & 23.96 & 4.31 & 33.03 & 36.93 & 11.29 & 18.75 & 5.95 & 52.83 & 17.68 & 23.54 & -27.08 & 15.90 & 6.39 & 4.79 \\
\hline Nueva Vizcaya & 2,536 & 28.74 & 30.03 & 1.30 & 2.56 & 33.68 & 28.86 & 34.90 & 1.58 & 31.07 & 28.98 & 38.37 & -0.99 & -2.60 & 0.12 & 3.47 \\
\hline Occidental Mindoro & 4,679 & 33.41 & 34.55 & 1.14 & 6.50 & 23.38 & 17.01 & 53.11 & 5.83 & 21.59 & 17.27 & 55.31 & -0.66 & -1.80 & 0.26 & 2.20 \\
\hline Oriental Mindoro & 2,914 & 39.21 & 39.05 & -0.16 & 0.86 & 11.43 & 18.63 & 69.08 & 1.10 & 12.53 & 18.22 & 68.15 & 0.24 & 1.10 & -0.41 & -0.93 \\
\hline Palawan & 6,715 & 44.62 & 44.03 & -0.59 & 0.27 & 7.92 & 13.67 & 78.14 & 0.66 & 8.92 & 13.72 & 76.71 & 0.39 & 1.00 & 0.04 & -1.43 \\
\hline Pampanga & 1,315 & 19.98 & 24.17 & 4.18 & 22.51 & 44.49 & 16.27 & 16.73 & 4.79 & 49.81 & 23.50 & 21.90 & -17.72 & 5.32 & 7.22 & 5.17 \\
\hline Pangasinan & 4,645 & 19.94 & 24.77 & 4.84 & 23.25 & 40.78 & 17.12 & 18.86 & 4.71 & 44.09 & 22.67 & 28.53 & -18.54 & 3.32 & 5.55 & 9.67 \\
\hline Quezon & 4,804 & 47.33 & 48.90 & 1.58 & 0.04 & 4.29 & 11.87 & 83.81 & 0.25 & 3.48 & 10.22 & 86.05 & 0.21 & -0.81 & -1.64 & 2.25 \\
\hline Quirino & 1,172 & 34.88 & 27.92 & -6.97 & 0.00 & 15.96 & 33.36 & 50.68 & 0.68 & 38.23 & 25.51 & 35.58 & 0.68 & 22.27 & -7.85 & -15.10 \\
\hline Rizal & 712 & 35.29 & 40.63 & 5.34 & 2.53 & 14.05 & 23.60 & 59.83 & 0.00 & 5.76 & 18.96 & 75.28 & -2.53 & -8.29 & -4.63 & 15.45 \\
\hline Romblon & 679 & 40.88 & 39.05 & -1.83 & 0.00 & 7.81 & 21.65 & 70.55 & 0.15 & 10.16 & 21.80 & 67.89 & 0.15 & 2.36 & 0.15 & -2.65 \\
\hline Samar & 2,786 & 41.93 & 42.16 & 0.23 & 0.00 & 10.30 & 19.06 & 70.64 & 0.14 & 10.77 & 16.12 & 72.97 & 0.14 & 0.47 & -2.94 & 2.33 \\
\hline Sarangani & 2,398 & 32.89 & 36.18 & 3.29 & 1.25 & 19.64 & 31.03 & 48.08 & 0.13 & 11.55 & 29.61 & 58.72 & -1.13 & -8.09 & -1.42 & 10.63 \\
\hline Siquijor & 264 & 27.37 & 33.49 & 6.12 & 1.89 & 22.73 & 41.29 & 34.09 & 0.00 & 9.47 & 35.23 & 55.30 & -1.89 & -13.26 & -6.06 & 21.21 \\
\hline Sorsogon & 1,282 & 44.90 & 45.00 & 0.10 & 0.86 & 7.96 & 11.54 & 79.64 & 0.86 & 6.71 & 12.95 & 79.49 & 0.00 & -1.25 & 1.40 & -0.16 \\
\hline South Cotabato & 3,095 & 30.76 & 33.85 & 3.09 & 2.10 & 22.65 & 33.96 & 41.29 & 0.16 & 9.11 & 38.84 & 51.89 & -1.94 & -13.54 & 4.88 & 10.60 \\
\hline Southern Leyte & 951 & 45.88 & 51.68 & 5.81 & 0.00 & 4.00 & 13.04 & 82.97 & 0.00 & 2.10 & 7.89 & 90.01 & 0.00 & -1.89 & -5.15 & 7.05 \\
\hline Sultan Kudarat & 3,226 & 34.54 & 35.52 & 0.98 & 0.68 & 20.71 & 31.68 & 46.93 & 0.06 & 16.18 & 34.66 & 49.10 & -0.62 & -4.53 & 2.98 & 2.17 \\
\hline Sulu & 647 & 52.73 & 55.62 & 2.89 & 0.15 & 0.46 & 1.55 & 97.84 & 0.46 & 0.00 & 0.62 & 98.92 & 0.31 & -0.46 & -0.93 & 1.08 \\
\hline Surigao del Norte & 563 & 44.35 & 46.79 & 2.43 & 0.18 & 11.37 & 14.39 & 74.07 & 0.00 & 7.28 & 15.28 & 77.44 & -0.18 & -4.09 & 0.89 & 3.37 \\
\hline Surigao del Sur & 1,735 & 52.76 & 56.79 & 4.03 & 0.00 & 5.36 & 6.74 & 87.90 & 0.00 & 3.69 & 7.32 & 88.99 & 0.00 & -1.67 & 0.58 & 1.10 \\
\hline Tarlac & 2,536 & 22.14 & 28.22 & 6.07 & 27.56 & 33.91 & 11.67 & 26.85 & 3.67 & 42.98 & 20.54 & 32.81 & -23.90 & 9.07 & 8.87 & 5.95 \\
\hline Tawi-Tawi & 218 & 57.33 & 60.71 & 3.38 & 0.00 & 0.92 & 4.13 & 94.95 & 0.00 & 0.00 & 0.92 & 99.08 & 0.00 & -0.92 & -3.21 & 4.13 \\
\hline Zambales & 2,712 & 23.68 & 28.20 & 4.52 & 23.41 & 26.81 & 18.81 & 30.97 & 6.31 & 30.24 & 26.07 & 37.39 & -17.11 & 3.43 & 7.26 & 6.42 \\
\hline Zamboanga del Norte & 4,723 & 37.92 & 41.08 & 3.16 & 0.15 & 7.94 & 25.26 & 66.65 & 0.13 & 2.82 & 17.07 & 79.99 & -0.02 & -5.12 & -8.19 & 13.34 \\
\hline Zamboanga del Sur & 3,952 & 32.97 & 37.05 & 4.09 & 0.68 & 17.18 & 29.18 & 52.96 & 0.03 & 8.40 & 24.39 & 67.18 & -0.66 & -8.78 & -4.78 & 14.22 \\
\hline Zamboanga Sibugay & 2,086 & 38.98 & 41.42 & 2.43 & 0.77 & 8.63 & 18.17 & 72.44 & 0.10 & 6.76 & 15.15 & 78.00 & -0.67 & -1.87 & -3.02 & 5.56 \\
\hline Philippines - All & 188,841 & 33.79 & 35.58 & 1.80 & 3.79 & 22.12 & 23.44 & 50.66 & 2.47 & 20.76 & 21.48 & 55.30 & -1.32 & -1.36 & -1.96 & 4.64 \\
\hline
\end{tabular}


Asia Pacific Region : Tree Cover on Agricultural Land (2010)

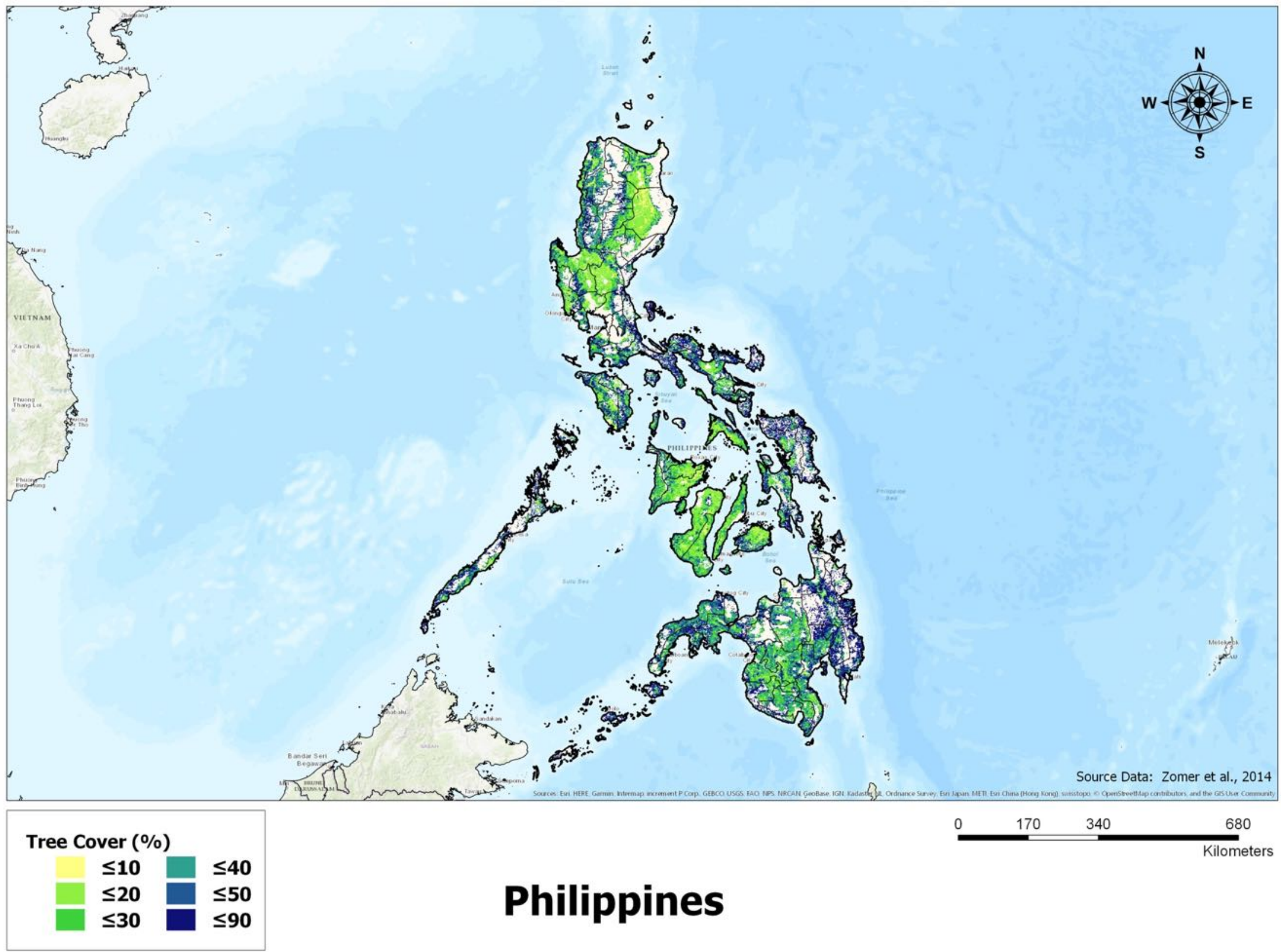


Asia Pacific Region : Change in Tree Cover on Agricultural Land (2000 - 2010)

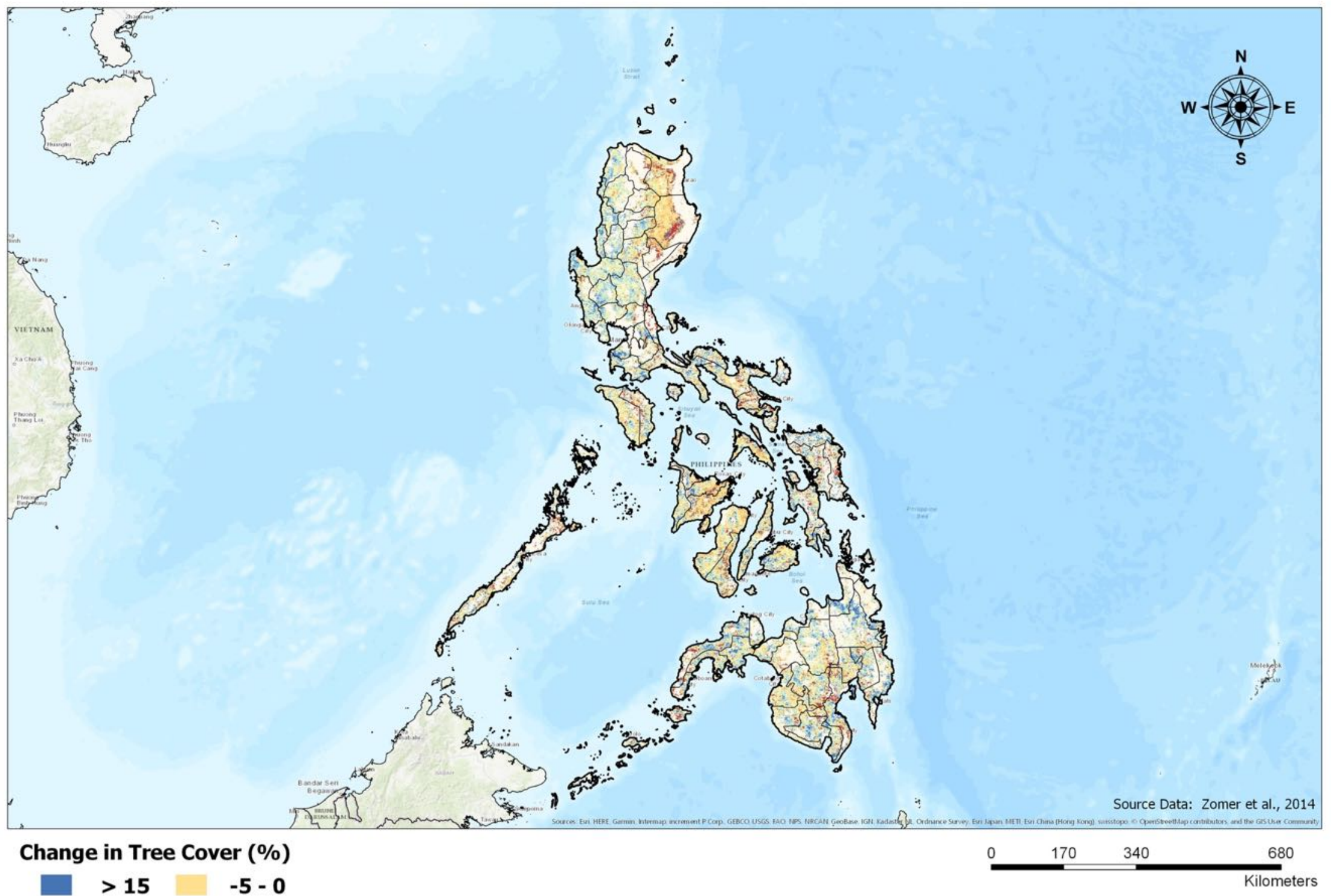

Philippines 


\section{Appendix 21: South Korea}

Average Percent Tree Cover by Province, and Areal Extent of Tree Cover Classes $\left(\mathrm{km}^{2}\right)$

\begin{tabular}{|c|c|c|c|c|c|c|c|c|c|c|c|c|c|c|c|c|}
\hline \multicolumn{2}{|c|}{ South Korea } & \multirow{3}{*}{\multicolumn{3}{|c|}{\begin{tabular}{|c|} 
Average Tree Cover by Province \\
Mean Tree Cover Percent by Province \\
$(\%)$
\end{tabular}}} & \multicolumn{12}{|c|}{ Areal Extent of Tree Cover Class $\left(\mathrm{km}^{2}\right)$} \\
\hline \multirow{3}{*}{ Province } & \multirow{3}{*}{ Area_km } & & & & \multirow{2}{*}{\multicolumn{4}{|c|}{$\begin{array}{c}\text { Year } 2000 \\
\text { Area by Tree Cover Class }\left(\mathrm{km}^{2}\right)\end{array}$}} & \multirow{2}{*}{\multicolumn{4}{|c|}{$\frac{\text { Year } 2010}{\text { Area by Tree Cover Class }\left(\mathrm{km}^{2}\right)}$}} & \multirow{2}{*}{\multicolumn{4}{|c|}{$\begin{array}{c}\text { Change (2000 to } 2010) \\
\text { Area by Tree Cover Class }\left(\mathrm{km}^{2}\right)\end{array}$}} \\
\hline & & & & & & & & & & & & & & & & \\
\hline & & 2000 & 2010 & Change & $<10 \%$ & $10-20 \%$ & $20-30 \%$ & $>30 \%$ & $<10 \%$ & $10-20 \%$ & $20-30 \%$ & $>30 \%$ & $<10 \%$ & $10-20 \%$ & $20-30 \%$ & $>30 \%$ \\
\hline Busan & 124 & 32.11 & 27.68 & -4.44 & 13 & 20 & 23 & 68 & 23 & 19 & 35 & 47 & 10 & -1 & 12 & -21 \\
\hline Chungcheongbuk-do & 1,917 & 27.30 & 26.77 & -0.53 & 355 & 432 & 355 & 775 & 436 & 383 & 324 & 774 & 81 & -49 & -31 & -1 \\
\hline Chungcheongnam-do & 4,333 & 22.97 & 23.48 & 0.51 & 1,065 & 1,121 & 871 & 1,276 & 983 & 1,151 & 899 & 1,300 & -82 & 30 & 28 & 24 \\
\hline Daegu & 117 & 32.29 & 30.43 & -1.86 & 10 & 29 & 18 & 60 & 13 & 28 & 20 & 56 & 3 & -1 & 2 & -4 \\
\hline Daejeon & 44 & 42.05 & 42.91 & 0.86 & 0 & 1 & 11 & 32 & 0 & 3 & 7 & 34 & 0 & 2 & -4 & 2 \\
\hline Gangwon-do & 823 & 35.57 & 38.92 & 3.34 & 43 & 125 & 161 & 494 & 45 & 116 & 103 & 559 & 2 & -9 & -58 & 65 \\
\hline Gwangju & 50 & 18.68 & 19.50 & 0.82 & 26 & 7 & 3 & 14 & 24 & 8 & 4 & 14 & -2 & 1 & 1 & 0 \\
\hline Gyeonggi-do & 1,923 & 18.74 & 18.00 & -0.73 & 661 & 613 & 311 & 338 & 754 & 546 & 301 & 322 & 93 & -67 & -10 & -16 \\
\hline Gyeongsangbuk-do & 6,322 & 32.76 & 33.45 & 0.69 & 667 & 1,017 & 1,128 & 3,510 & 781 & 956 & 942 & 3,643 & 114 & -61 & -186 & 133 \\
\hline Gyeongsangnam-do & 4,007 & 33.91 & 33.75 & -0.16 & 352 & 643 & 744 & 2,268 & 433 & 634 & 675 & 2,265 & 81 & -9 & -69 & -3 \\
\hline Incheon & 107 & 28.73 & 29.03 & 0.30 & 10 & 29 & 22 & 46 & 24 & 15 & 22 & 46 & 14 & -14 & 0 & 0 \\
\hline Jeju & 342 & 29.19 & 29.46 & 0.27 & 9 & 67 & 143 & 123 & 4 & 59 & 150 & 129 & -5 & -8 & 7 & 6 \\
\hline Jeollabuk-do & 3,917 & 25.41 & 24.61 & -0.80 & 1,309 & 672 & 485 & 1,451 & 1,312 & 738 & 495 & 1,372 & 3 & 66 & 10 & -79 \\
\hline Jeollanam-do & 6,456 & 27.35 & 29.14 & 1.80 & 1,399 & 1,440 & 1,043 & 2,574 & 1,331 & 1,339 & 954 & 2,832 & -68 & -101 & -89 & 258 \\
\hline Sejong & 196 & 24.07 & 22.24 & -1.83 & 32 & 48 & 58 & 58 & 53 & 38 & 52 & 53 & 21 & -10 & -6 & -5 \\
\hline Ulsan & 155 & 30.91 & 29.98 & -0.93 & 16 & 30 & 31 & 78 & 21 & 35 & 29 & 70 & 5 & 5 & -2 & -8 \\
\hline South Korea - All & 30,833 & 28.20 & 28.64 & 0.44 & 5,967 & 6,294 & 5,407 & 13,165 & 6,237 & 6,068 & 5,012 & 13,516 & 270 & -226 & -395 & 351 \\
\hline
\end{tabular}

Average Percent Tree Cover by Province, and Areal Extent of Tree Cover Classes as a Percent of the Total Agricultural Area (\%)

\begin{tabular}{|c|c|c|c|c|c|c|c|c|c|c|c|c|c|c|c|c|}
\hline \multicolumn{2}{|c|}{ South Korea } & \multirow{3}{*}{\multicolumn{3}{|c|}{$\begin{array}{c}\text { Average Tree Cover by Province } \\
\text { Percent Tree Cover } \\
(\%)\end{array}$}} & \multicolumn{12}{|c|}{ Areal Extent of Tree Cover Class as a Percent of Total Agricultural Area (\%) } \\
\hline \multirow{3}{*}{ Province } & \multirow{3}{*}{ Area_km } & & & & \multirow{2}{*}{\multicolumn{4}{|c|}{$\begin{array}{c}\text { Year } 2000 \\
\text { Percent of Total Agrciultural Area (\%) }\end{array}$}} & \multirow{2}{*}{\multicolumn{4}{|c|}{$\begin{array}{c}\text { Year } 2010 \\
\text { Percent of Total Agrciultural Area (\%) }\end{array}$}} & \multirow{2}{*}{\multicolumn{4}{|c|}{$\begin{array}{c}\text { Change (2000 to 2010) } \\
\end{array}$}} \\
\hline & & & & & & & & & & & & & & & & \\
\hline & & 2000 & 2010 & Change & $<10 \%$ & $10-20 \%$ & $20-30 \%$ & $>30 \%$ & $<10 \%$ & $10-20 \%$ & $20-30 \%$ & $>30 \%$ & $<10 \%$ & $10-20 \%$ & $20-30 \%$ & $>30 \%$ \\
\hline Busan & 124 & 32.11 & 27.68 & -4.44 & 10.48 & 16.13 & 18.55 & 54.84 & 18.55 & 15.32 & 28.23 & 37.90 & 8.06 & -0.81 & 9.68 & -16.94 \\
\hline Chungcheongbuk-do & 1.917 & 27.30 & 26.77 & -0.53 & 18.52 & 22.54 & 18.52 & 40.43 & 22.74 & 19.98 & 16.90 & 40.38 & 4.23 & -2.56 & -1.62 & -0.05 \\
\hline Chungcheongnam-do & 4,333 & 22.97 & 23.48 & 0.51 & 24.58 & 25.87 & 20.10 & 29.45 & 22.69 & 26.56 & 20.75 & 30.00 & -1.89 & 0.69 & 0.65 & 0.55 \\
\hline Daegu & 117 & 32.29 & 30.43 & -1.86 & 8.55 & 24.79 & 15.38 & 51.28 & 11.11 & 23.93 & 17.09 & 47.86 & 2.56 & -0.85 & 1.71 & -3.42 \\
\hline Daejeon & 44 & 42.05 & 42.91 & 0.86 & 0.00 & 2.27 & 25.00 & 72.73 & 0.00 & 6.82 & 15.91 & 77.27 & 0.00 & 4.55 & -9.09 & 4.55 \\
\hline Gangwon-do & 823 & 35.57 & 38.92 & 3.34 & 5.22 & 15.19 & 19.56 & 60.02 & 5.47 & 14.09 & 12.52 & 67.92 & 0.24 & -1.09 & -7.05 & 7.90 \\
\hline Gwangju & 50 & 18.68 & 19.50 & 0.82 & 52.00 & 14.00 & 6.00 & 28.00 & 48.00 & 16.00 & 8.00 & 28.00 & -4.00 & 2.00 & 2.00 & 0.00 \\
\hline Gyeonggi-do & 1,923 & 18.74 & 18.00 & -0.73 & 34.37 & 31.88 & 16.17 & 17.58 & 39.21 & 28.39 & 15.65 & 16.74 & 4.84 & -3.48 & -0.52 & -0.83 \\
\hline Gyeongsangbuk-do & 6,322 & 32.76 & 33.45 & 0.69 & 10.55 & 16.09 & 17.84 & 55.52 & 12.35 & 15.12 & 14.90 & 57.62 & 1.80 & -0.96 & -2.94 & 2.10 \\
\hline Gyeongsangnam-do & 4,007 & 33.91 & 33.75 & -0.16 & 8.78 & 16.05 & 18.57 & 56.60 & 10.81 & 15.82 & 16.85 & 56.53 & 2.02 & -0.22 & -1.72 & -0.07 \\
\hline Incheon & 107 & 28.73 & 29.03 & 0.30 & 9.35 & 27.10 & 20.56 & 42.99 & 22.43 & 14.02 & 20.56 & 42.99 & 13.08 & -13.08 & 0.00 & 0.00 \\
\hline Jeju & 342 & 29.19 & 29.46 & 0.27 & 2.63 & 19.59 & 41.81 & 35.97 & 1.17 & 17.25 & 43.86 & 37.72 & -1.46 & -2.34 & 2.05 & 1.75 \\
\hline Jeollabuk-do & 3,917 & 25.41 & 24.61 & -0.80 & 33.42 & 17.16 & 12.38 & 37.04 & 33.50 & 18.84 & 12.64 & 35.03 & 0.08 & 1.68 & 0.26 & -2.02 \\
\hline Jeollanam-do & 6,456 & 27.35 & 29.14 & 1.80 & 21.67 & 22.31 & 16.16 & 39.87 & 20.62 & 20.74 & 14.78 & 43.87 & -1.05 & -1.56 & -1.38 & 4.00 \\
\hline Sejong & 196 & 24.07 & 22.24 & -1.83 & 16.33 & 24.49 & 29.59 & 29.59 & 27.04 & 19.39 & 26.53 & 27.04 & 10.71 & -5.10 & -3.06 & -2.55 \\
\hline Ulsan & 155 & 30.91 & 29.98 & -0.93 & 10.32 & 19.35 & 20.00 & 50.32 & 13.55 & 22.58 & 18.71 & 45.16 & 3.23 & 3.23 & -1.29 & -5.16 \\
\hline South Korea - All & 30,833 & 28.20 & 28.64 & 0.44 & 19.35 & 20.41 & 17.54 & 42.70 & 20.23 & 19.68 & 16.26 & 43.84 & 0.88 & -0.73 & -1.28 & 1.14 \\
\hline
\end{tabular}


Asia Pacific Region : Tree Cover on Agricultural Land (2010)

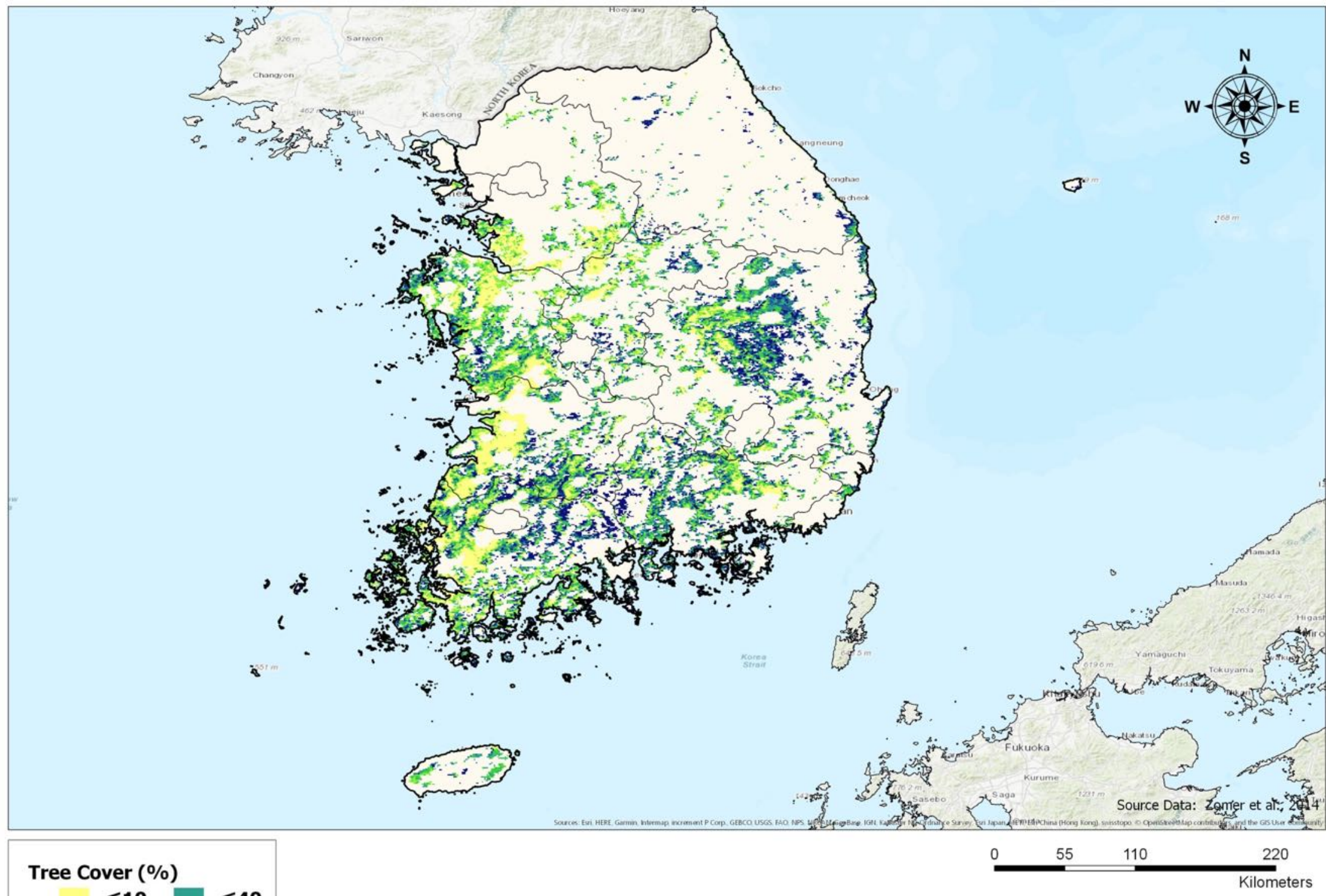

South Korea 
Asia Pacific Region : Change in Tree Cover on Agricultural Land (2000 - 2010)

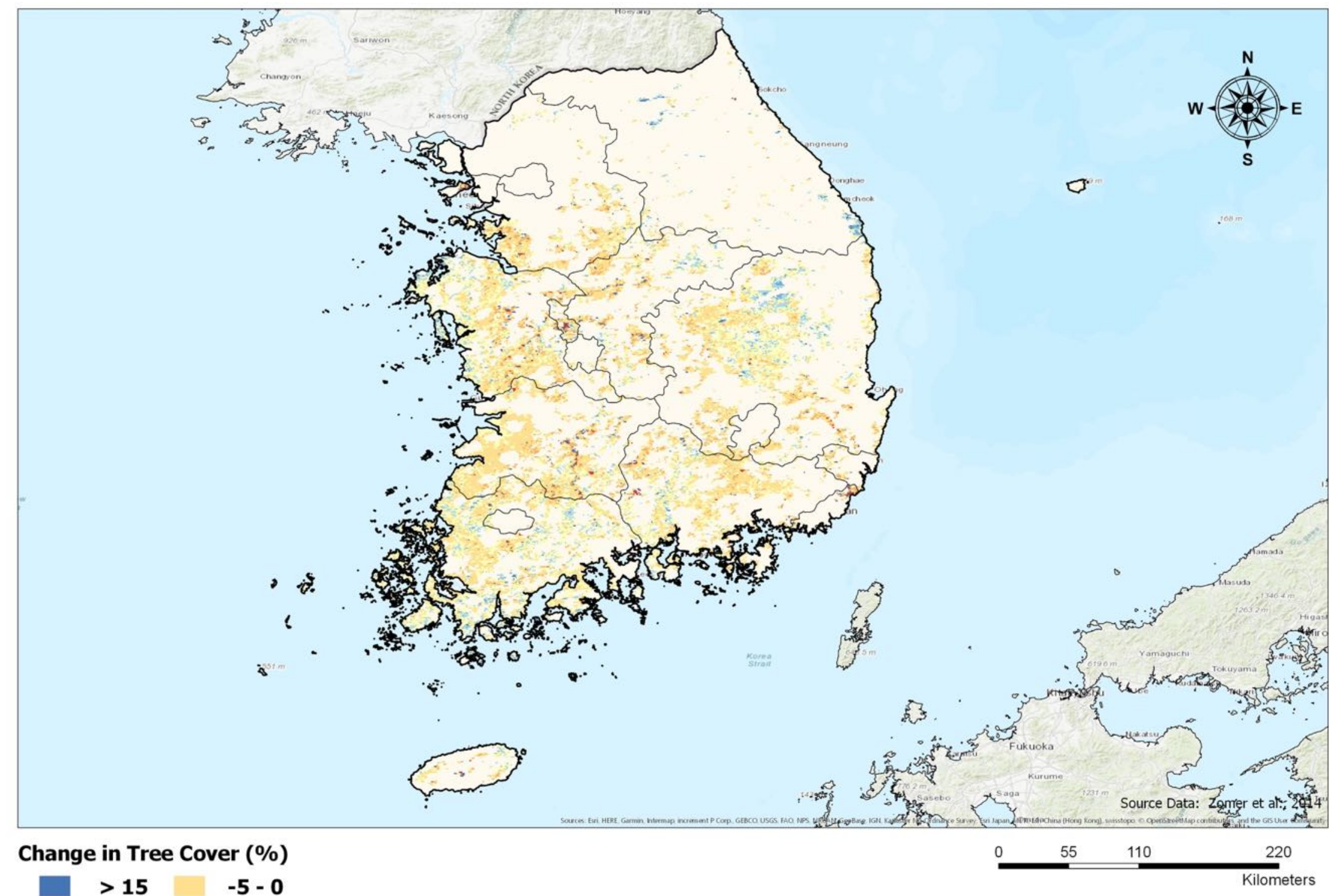

$10-15$

$5-10-15--10$

$0-5 \square \leq-15$

South Korea 


\section{Appendix 22: Sri Lanka}

Average Percent Tree Cover by Province, and Areal Extent of Tree Cover Classes $\left(\mathrm{km}^{2}\right)$

\begin{tabular}{|c|c|c|c|c|c|c|c|c|c|c|c|c|c|c|c|c|}
\hline \multicolumn{2}{|c|}{ Sri Lanka } & \multicolumn{3}{|c|}{ Average Tree Cover by Province } & \multicolumn{12}{|c|}{ Areal Extent of Tree Cover Class $\left(\mathrm{km}^{2}\right)$} \\
\hline \multirow{3}{*}{ Province } & \multirow{3}{*}{ Area_km } & \multirow{2}{*}{\multicolumn{3}{|c|}{$\begin{array}{c}\text { Mean Tree Cover Percent by Province } \\
(\%)\end{array}$}} & \multirow{2}{*}{\multicolumn{4}{|c|}{$\begin{array}{c}\text { Year } 2000 \\
\text { Area by Tree Cover Class }\left(\mathrm{km}^{2}\right)\end{array}$}} & \multirow{2}{*}{\multicolumn{4}{|c|}{$\begin{array}{c}\text { Year } 2010 \\
\text { Area by Tree Cover Class }\left(\mathrm{km}^{2}\right)\end{array}$}} & \multirow{2}{*}{\multicolumn{4}{|c|}{$\begin{array}{l}\text { Change (2000 to 2010) } \\
\text { rea }\end{array}$}} \\
\hline & & & & & & & & & & & & & & & & \\
\hline & & 2000 & 2010 & Change & $<10 \%$ & $10-20 \%$ & $20-30 \%$ & $>\mathbf{3 0} \%$ & $<10 \%$ & $10-20 \%$ & $20-30 \%$ & $>30 \%$ & $<10 \%$ & $10-20 \%$ & $20-30 \%$ & $>30 \%$ \\
\hline Ampara & 1,827 & 14.90 & 17.19 & 2.29 & 735 & 758 & 178 & 156 & 265 & 1,124 & 297 & 141 & -470 & 366 & 119 & -15 \\
\hline Anuradhapura & 2,449 & 19.77 & 21.93 & 2.16 & 377 & 1,280 & 401 & 391 & 76 & 1,446 & 511 & 416 & -301 & 166 & 110 & 25 \\
\hline Badulla & 790 & 22.72 & 28.05 & 5.34 & 118 & 295 & 182 & 195 & 2 & 286 & 212 & 290 & -116 & -9 & 30 & 95 \\
\hline Batticaloa & 1,414 & 10.87 & 14.60 & 3.73 & 874 & 436 & 75 & 29 & 487 & 660 & 193 & 74 & -387 & 224 & 118 & 45 \\
\hline Colombo & 176 & 49.31 & 40.21 & -9.10 & 0 & 1 & 10 & 165 & 0 & 16 & 43 & 117 & 0 & 15 & 33 & -48 \\
\hline Galle & 1,014 & 43.11 & 37.32 & -5.79 & 0 & 5 & 110 & 899 & 0 & 39 & 300 & 675 & 0 & 34 & 190 & -224 \\
\hline Gampaha & 601 & 41.29 & 34.31 & -6.98 & 0 & 5 & 53 & 543 & 0 & 31 & 196 & 374 & 0 & 26 & 143 & -169 \\
\hline Hambantota & 902 & 23.06 & 27.55 & 4.49 & 132 & 364 & 183 & 223 & 70 & 289 & 223 & 320 & -62 & -75 & 40 & 97 \\
\hline Jaffna & 484 & 9.35 & 7.34 & -2.01 & 333 & 128 & 20 & 3 & 407 & 61 & 8 & 8 & 74 & -67 & -12 & 5 \\
\hline Kalutara & 1,100 & 48.48 & 36.08 & -12.41 & 1 & 3 & 51 & 1,045 & 1 & 98 & 275 & 726 & 0 & 95 & 224 & -319 \\
\hline Kandy & 895 & 38.79 & 42.57 & 3.79 & 12 & 72 & 160 & 651 & 0 & 55 & 115 & 725 & -12 & -17 & -45 & 74 \\
\hline Kegalle & 1,165 & 52.94 & 53.18 & 0.24 & 0 & 0 & 8 & 1,157 & 0 & 2 & 22 & 1,141 & 0 & 2 & 14 & -16 \\
\hline Kilinochchi & 600 & 17.10 & 16.33 & -0.76 & 336 & 120 & 33 & 111 & 271 & 184 & 51 & 94 & -65 & 64 & 18 & -17 \\
\hline Kurunegala & 4,463 & 27.25 & 28.23 & 0.98 & 30 & 1,253 & 1,814 & 1,366 & 6 & 932 & 2,159 & 1,366 & -24 & -321 & 345 & 0 \\
\hline Mannar & 461 & 14.31 & 13.77 & -0.54 & 257 & 107 & 42 & 55 & 242 & 139 & 40 & 40 & -15 & 32 & -2 & -15 \\
\hline Matale & 783 & 29.90 & 35.68 & 5.78 & 60 & 261 & 138 & 324 & 13 & 160 & 183 & 427 & -47 & -101 & 45 & 103 \\
\hline Matara & 726 & 48.26 & 46.02 & -2.24 & 0 & 2 & 46 & 678 & 0 & 16 & 78 & 632 & 0 & 14 & 32 & -46 \\
\hline Moneragala & 1,512 & 20.00 & 23.36 & 3.36 & 435 & 497 & 290 & 290 & 113 & 701 & 309 & 389 & -322 & 204 & 19 & 99 \\
\hline Mullaitivu & 627 & 24.59 & 23.24 & -1.35 & 126 & 211 & 86 & 204 & 77 & 284 & 87 & 179 & -49 & 73 & 1 & -25 \\
\hline Nuwara Eliya & 612 & 30.54 & 34.48 & 3.93 & 2 & 88 & 281 & 241 & 1 & 29 & 207 & 375 & -1 & -59 & -74 & 134 \\
\hline Polonnaruwa & 1,686 & 14.69 & 18.87 & 4.18 & 588 & 838 & 139 & 121 & 74 & 1,200 & 221 & 191 & -514 & 362 & 82 & 70 \\
\hline Puttalam & 1,855 & 22.30 & 23.40 & 1.10 & 306 & 626 & 507 & 416 & 212 & 578 & 653 & 412 & -94 & -48 & 146 & -4 \\
\hline Ratnapura & 1,546 & 42.95 & 45.97 & 3.02 & 16 & 154 & 180 & 1,196 & 0 & 51 & 146 & 1,349 & -16 & -103 & -34 & 153 \\
\hline Trincomalee & 1,065 & 17.68 & 19.74 & 2.06 & 331 & 440 & 140 & 154 & 179 & 528 & 179 & 179 & -152 & 88 & 39 & 25 \\
\hline Vavuniya & 288 & 29.53 & 28.66 & -0.88 & 5 & 103 & 55 & 125 & 9 & 92 & 61 & 126 & 4 & -11 & 6 & 1 \\
\hline Sri Lanka - All & 29,041 & 26.94 & 27.95 & 1.01 & 5,074 & 8,047 & $\begin{array}{l}5,182 \\
\end{array}$ & 10,738 & 2,505 & 9,001 & 6,769 & 10,766 & $-2,569$ & 954 & 1,587 & 28 \\
\hline
\end{tabular}


Average Percent Tree Cover by Province, and Areal Extent of Tree Cover Classes as a Percent of the Total Agricultural Area (\%)

\begin{tabular}{|c|c|c|c|c|c|c|c|c|c|c|c|c|c|c|c|c|}
\hline \multicolumn{2}{|c|}{ Sri Lanka } & \multirow{3}{*}{\multicolumn{3}{|c|}{$\begin{array}{c}\text { Average Tree Cover by Province } \\
\text { Percent Tree Cover } \\
(\%)\end{array}$}} & \multicolumn{12}{|c|}{ Areal Extent of Tree Cover Class as a Percent of Total Agricultural Area (\%) } \\
\hline \multirow{3}{*}{ Province } & \multirow{3}{*}{ Area_km } & & & & \multirow{2}{*}{\multicolumn{4}{|c|}{$\begin{array}{c}\text { Year } 2000 \\
\text { Percent of Total Agrciultural Area }(\%)\end{array}$}} & \multirow{2}{*}{\multicolumn{4}{|c|}{$\begin{array}{c}\text { Year 2010 } \\
\text { Percent of Total Agrciultural Area (\%) }\end{array}$}} & \multirow{2}{*}{\multicolumn{4}{|c|}{ Percent of Total Agrciultural Area (\%) }} \\
\hline & & & & & & & & & & & & & & & & \\
\hline & & 2000 & 2010 & Change & $<10 \%$ & $10-20 \%$ & $20-30 \%$ & $>30 \%$ & $<10 \%$ & $10-20 \%$ & $20-30 \%$ & $>30 \%$ & $<10 \%$ & $10-20 \%$ & $20-30 \%$ & $>30 \%$ \\
\hline Ampara & 1,827 & 14.90 & 17.19 & 2.29 & 40.23 & 41.49 & 9.74 & 8.54 & 14.50 & 61.52 & 16.26 & 7.72 & -25.73 & 20.03 & 6.51 & -0.82 \\
\hline Anuradhapura & 2,449 & 19.77 & 21.93 & 2.16 & 15.39 & 52.27 & 16.37 & 15.97 & 3.10 & 59.04 & 20.87 & 16.99 & -12.29 & 6.78 & 4.49 & 1.02 \\
\hline Badulla & 790 & 22.72 & 28.05 & 5.34 & 14.94 & 37.34 & 23.04 & 24.68 & 0.25 & 36.20 & 26.84 & 36.71 & -14.68 & -1.14 & 3.80 & 12.03 \\
\hline Batticaloa & 1,414 & 10.87 & 14.60 & 3.73 & 61.81 & 30.83 & 5.30 & 2.05 & 34.44 & 46.68 & 13.65 & 5.23 & -27.37 & 15.84 & 8.35 & 3.18 \\
\hline Colombo & 176 & 49.31 & 40.21 & -9.10 & 0.00 & 0.57 & 5.68 & 93.75 & 0.00 & 9.09 & 24.43 & 66.48 & 0.00 & 8.52 & 18.75 & -27.27 \\
\hline Galle & 1,014 & 43.11 & 37.32 & -5.79 & 0.00 & 0.49 & 10.85 & 88.66 & 0.00 & 3.85 & 29.59 & 66.57 & 0.00 & 3.35 & 18.74 & -22.09 \\
\hline Gampaha & 601 & 41.29 & 34.31 & -6.98 & 0.00 & 0.83 & 8.82 & 90.35 & 0.00 & 5.16 & 32.61 & 62.23 & 0.00 & 4.33 & 23.79 & -28.12 \\
\hline Hambantota & 902 & 23.06 & 27.55 & 4.49 & 14.63 & 40.36 & 20.29 & 24.72 & 7.76 & 32.04 & 24.72 & 35.48 & -6.87 & -8.31 & 4.43 & 10.75 \\
\hline Jaffna & 484 & 9.35 & 7.34 & -2.01 & 68.80 & 26.45 & 4.13 & 0.62 & 84.09 & 12.60 & 1.65 & 1.65 & 15.29 & -13.84 & -2.48 & 1.03 \\
\hline Kalutara & 1.100 & 48.48 & 36.08 & -12.41 & 0.09 & 0.27 & 4.64 & 95.00 & 0.09 & 8.91 & 25.00 & 66.00 & 0.00 & 8.64 & 20.36 & -29.00 \\
\hline Kandy & 895 & 38.79 & 42.57 & 3.79 & 1.34 & 8.04 & 17.88 & 72.74 & 0.00 & 6.15 & 12.85 & 81.01 & -1.34 & -1.90 & -5.03 & 8.27 \\
\hline Kegalle & 1,165 & 52.94 & 53.18 & 0.24 & 0.00 & 0.00 & 0.69 & 99.31 & 0.00 & 0.17 & 1.89 & 97.94 & 0.00 & 0.17 & 1.20 & -1.37 \\
\hline Kilinochchi & 600 & 17.10 & 16.33 & -0.76 & 56.00 & 20.00 & 5.50 & 18.50 & 45.17 & 30.67 & 8.50 & 15.67 & -10.83 & 10.67 & 3.00 & -2.83 \\
\hline Kurunegala & 4,463 & 27.25 & 28.23 & 0.98 & 0.67 & 28.08 & 40.65 & 30.61 & 0.13 & 20.88 & 48.38 & 30.61 & -0.54 & -7.19 & 7.73 & 0.00 \\
\hline Mannar & 461 & 14.31 & 13.77 & -0.54 & 55.75 & 23.21 & 9.11 & 11.93 & 52.49 & 30.15 & 8.68 & 8.68 & -3.25 & 6.94 & -0.43 & -3.25 \\
\hline $\begin{array}{l}\text { Matale } \\
\text { Mal }\end{array}$ & 783 & 29.90 & 35.68 & 5.78 & 7.66 & 33.33 & 17.62 & 41.38 & 1.66 & 20.43 & 23.37 & 54.53 & -6.00 & -12.90 & 5.75 & 13.15 \\
\hline Matara & 726 & 48.26 & 46.02 & -2.24 & 0.00 & 0.28 & 6.34 & 93.39 & 0.00 & 2.20 & 10.74 & 87.05 & 0.00 & 1.93 & 4.41 & -6.34 \\
\hline Moneragala & 1,512 & 20.00 & 23.36 & 3.36 & 28.77 & 32.87 & 19.18 & 19.18 & 7.47 & 46.36 & 20.44 & 25.73 & -21.30 & 13.49 & 1.26 & 6.55 \\
\hline Mullaitivu & 627 & 24.59 & 23.24 & -1.35 & 20.10 & 33.65 & 13.72 & 32.54 & 12.28 & 45.30 & 13.88 & 28.55 & -7.82 & 11.64 & 0.16 & -3.99 \\
\hline Nuwara Eliya & 612 & 30.54 & 34.48 & 3.93 & 0.33 & 14.38 & 45.92 & 39.38 & 0.16 & 4.74 & 33.82 & 61.27 & -0.16 & -9.64 & -12.09 & 21.90 \\
\hline Polonnaruwa & 1,686 & 14.69 & 18.87 & 4.18 & 34.88 & 49.70 & 8.24 & 7.18 & 4.39 & 71.17 & 13.11 & 11.33 & -30.49 & 21.47 & 4.86 & 4.15 \\
\hline Puttalam & 1,855 & 22.30 & 23.40 & 1.10 & 16.50 & 33.75 & 27.33 & 22.43 & 11.43 & 31.16 & 35.20 & 22.21 & -5.07 & -2.59 & 7.87 & $\begin{array}{l}-0.22 \\
-10\end{array}$ \\
\hline Ratnapura & 1,546 & 42.95 & 45.97 & 3.02 & 1.03 & 9.96 & 11.64 & 77.36 & 0.00 & 3.30 & 9.44 & 87.26 & -1.03 & -6.66 & -2.20 & 9.90 \\
\hline Trincomalee & 1,065 & 17.68 & 19.74 & 2.06 & 31.08 & 41.31 & 13.15 & 14.46 & 16.81 & 49.58 & 16.81 & 16.81 & -14.27 & 8.26 & 3.66 & 2.35 \\
\hline Vavuniya & 288 & 29.53 & 28.66 & -0.88 & 1.74 & 35.76 & 19.10 & 43.40 & 3.13 & 31.94 & 21.18 & 43.75 & 1.39 & -3.82 & 2.08 & 0.35 \\
\hline Sri Lanka - All & 29,041 & 26.94 & 27.95 & 1.01 & 17.47 & 27.71 & 17.84 & 36.98 & 8.63 & 30.99 & 23.31 & 37.07 & -8.85 & 3.29 & 5.46 & 0.10 \\
\hline
\end{tabular}


Asia Pacific Region : Tree Cover on Agricultural Land (2010)

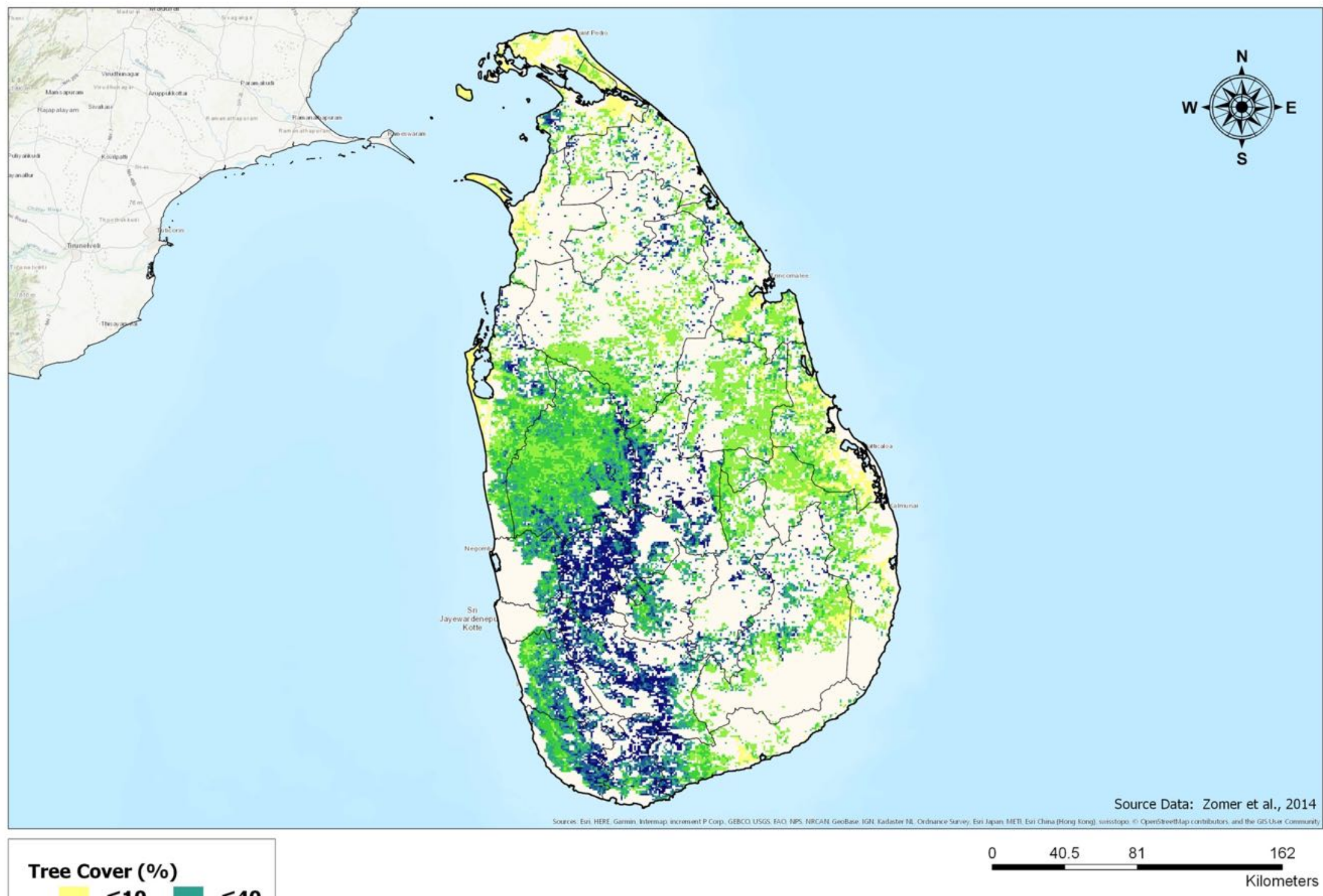

Sri Lanka 
Asia Pacific Region : Change in Tree Cover on Agricultural Land (2000 - 2010)

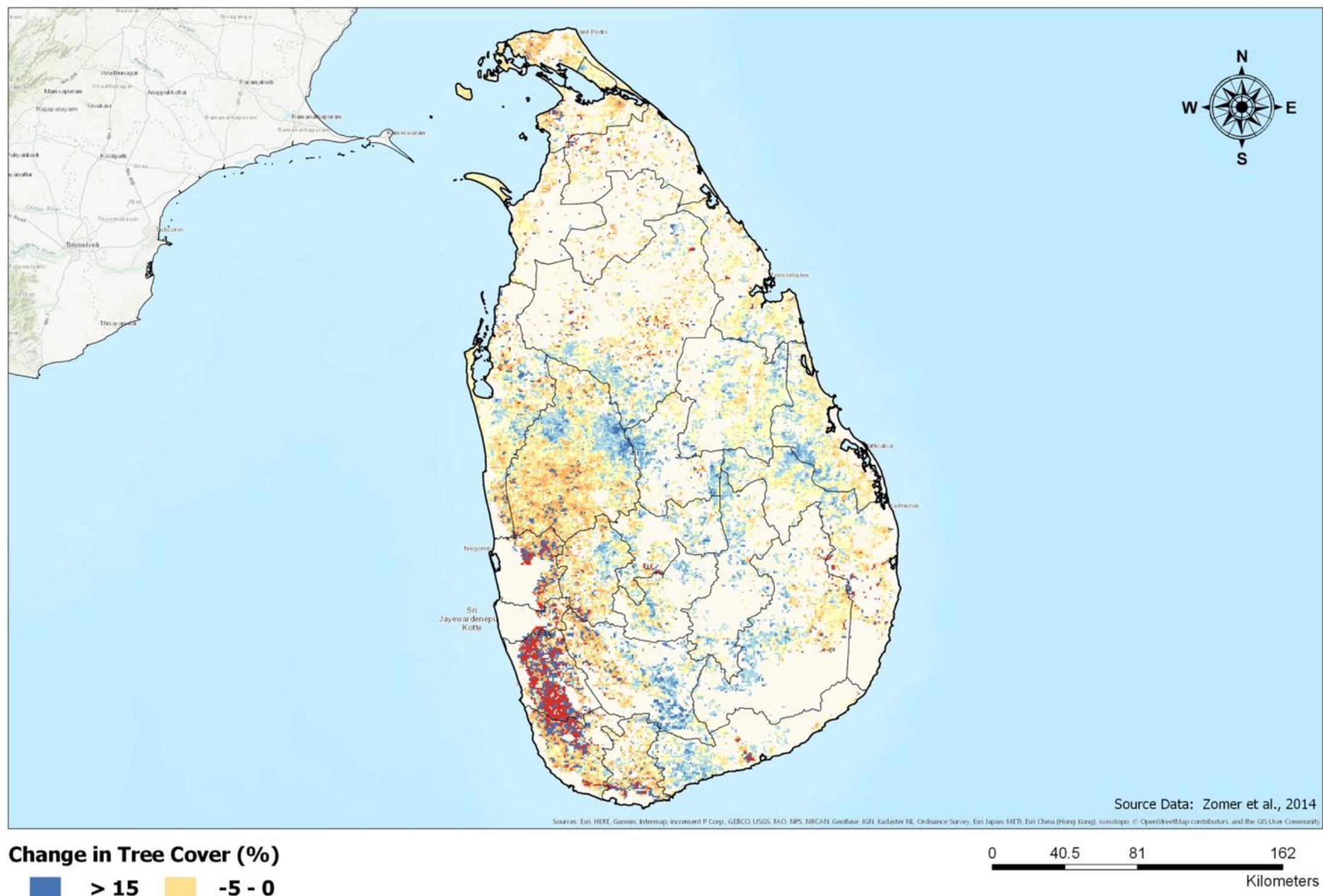

Sri Lanka

$5-10-15--10$

$0-5 \square \leq-15$ 
Appendix 23: Thailand

Average Percent Tree Cover by Province, and Areal Extent of Tree Cover Classes $\left(\mathrm{km}^{2}\right)$

\begin{tabular}{|c|c|c|c|c|c|c|c|c|c|c|c|c|c|c|c|c|}
\hline \multicolumn{2}{|l|}{ Thailand } & \multirow{3}{*}{\multicolumn{3}{|c|}{\begin{tabular}{|c|} 
Average Tree Cover by Province \\
Mean Tree Cover Percent by Province \\
$(\%)$
\end{tabular}}} & \multicolumn{12}{|c|}{ Areal Extent of Tree Cover Class $\left(\mathrm{km}^{2}\right)$} \\
\hline \multirow{3}{*}{ Province } & \multirow{3}{*}{ Area_km } & & & & \multicolumn{4}{|c|}{ Year 2000} & \multirow{2}{*}{\multicolumn{4}{|c|}{$\begin{array}{c}\text { Year } 2010 \\
\text { Area by Tree Cover Class }\left(\mathrm{km}^{2}\right)\end{array}$}} & \multicolumn{4}{|c|}{ Change (2000 to 2010) } \\
\hline & & & & & & by Tree $\mathrm{C}$ & er Class $(\mathbf{k}$ & & & & & & & a by Tree & over Class ( $k$ & \\
\hline & & 2000 & 2010 & Change & $<10 \%$ & $10-20 \%$ & $20-30 \%$ & $>30 \%$ & $<10 \%$ & $10-20 \%$ & $20-30 \%$ & $>30 \%$ & $<10 \%$ & $10-20 \%$ & $20-30 \%$ & $>30 \%$ \\
\hline Amnat Charoen & 2,468 & 6.50 & 4.94 & -1.56 & 2,103 & 160 & 95 & 110 & 2,183 & 193 & 61 & 31 & 80 & 33 & -34 & -79 \\
\hline Ang Thong & 815 & 14.73 & 16.59 & 1.86 & 82 & 695 & 38 & 0 & 25 & 730 & 60 & 0 & -57 & 35 & 22 & 0 \\
\hline Bangkok Metropolis & 211 & 12.08 & 16.64 & 4.56 & 66 & 143 & 2 & 0 & 33 & 139 & 39 & 0 & -33 & -4 & 37 & 0 \\
\hline Bueng Kan & 3,068 & 7.35 & 15.93 & 8.59 & 2,563 & 377 & 79 & 49 & 1,054 & 1,218 & 552 & 244 & $-1,509$ & 841 & 473 & 195 \\
\hline Buri Ram & 7,114 & 4.19 & 6.21 & 2.01 & 6,774 & 266 & 48 & 26 & 6,377 & 534 & 135 & 68 & -397 & 268 & 87 & 42 \\
\hline Chachoengsao & 2,873 & 8.54 & 15.45 & 6.91 & 2,115 & 664 & 68 & 26 & 632 & 1,791 & 335 & 115 & $-1,483$ & 1,127 & 267 & 89 \\
\hline Chai Nat & 2,052 & 11.60 & 13.50 & 1.90 & 896 & 1,033 & 114 & 9 & 652 & 1,221 & 172 & 7 & -244 & 188 & 58 & -2 \\
\hline Chaiyaphum & 7,352 & 5.99 & 9.21 & 3.22 & 6,342 & 588 & 243 & 179 & 5,365 & 1,364 & 285 & 338 & -977 & 776 & 42 & 159 \\
\hline Chanthaburi & 2,331 & 22.61 & 20.96 & -1.64 & 321 & 790 & 720 & 500 & 193 & 1,133 & 685 & 320 & -128 & 343 & -35 & -180 \\
\hline Chiang Mai & 3,756 & 32.72 & 31.41 & -1.31 & 479 & 705 & 666 & 1,906 & 307 & 878 & 870 & 1,701 & -172 & 173 & 204 & -205 \\
\hline Chiang Rai & 4,944 & 27.32 & 24.22 & -3.10 & 1,660 & 785 & 484 & 2,015 & 1,669 & 1,064 & 576 & 1,635 & 9 & 279 & 92 & -380 \\
\hline Chon Buri & 2,599 & 10.91 & 13.95 & 3.05 & 1,774 & 568 & 121 & 136 & 1,156 & 922 & 339 & 182 & -618 & 354 & 218 & 46 \\
\hline Chumphon & 3,693 & 40.06 & 41.24 & 1.18 & 14 & 248 & 781 & 2,650 & 5 & 141 & 582 & 2,965 & -9 & -107 & -199 & 315 \\
\hline Kalasin & 5,631 & 3.32 & 4.84 & 1.51 & 5,381 & 166 & 56 & 28 & 5,106 & 376 & 87 & 62 & -275 & 210 & 31 & 34 \\
\hline Kamphaeng Phet & 5,210 & 11.85 & 12.17 & 0.32 & 2,806 & 2,055 & 135 & 214 & 2,565 & 2,284 & 141 & 220 & -241 & 229 & 6 & 6 \\
\hline Kanchanaburi & 5,692 & 19.10 & 20.22 & 1.12 & 3,077 & 867 & 368 & 1,380 & 2,490 & 1,205 & 552 & 1,445 & -587 & 338 & 184 & 65 \\
\hline Khon Kaen & 8,122 & 3.95 & 5.15 & 1.20 & 7,588 & 256 & 135 & 143 & 7,286 & 453 & 151 & 232 & -302 & 197 & 16 & 89 \\
\hline Krabi & 3,501 & 42.35 & 46.89 & 4.53 & 2 & 132 & 559 & 2,808 & 0 & 46 & 236 & 3,219 & -2 & -86 & -323 & 411 \\
\hline Lampang & 2,202 & 29.08 & 28.20 & -0.88 & 501 & 525 & 338 & 838 & 443 & 585 & 355 & 819 & -58 & 60 & 17 & -19 \\
\hline Lamphun & 987 & 13.65 & 16.40 & 2.74 & 500 & 288 & 117 & 82 & 291 & 449 & 175 & 72 & -209 & 161 & 58 & -10 \\
\hline Loei & 5,050 & 18.62 & 20.68 & 2.05 & 1,536 & 1,749 & 978 & 787 & 1,087 & 1,866 & 1,094 & 1,003 & -449 & 117 & 116 & 216 \\
\hline Lop Buri & 4,816 & 7.71 & 13.19 & 5.47 & 3,636 & 1,077 & 94 & 9 & 1,562 & 2,815 & 359 & 80 & $-2,074$ & 1,738 & 265 & 71 \\
\hline Mae Hong Son & 1,626 & 48.10 & 44.32 & -3.78 & 4 & 39 & 69 & 1,514 & 28 & 79 & 182 & 1,337 & 24 & 40 & 113 & -177 \\
\hline Maha Sarakham & 5,106 & 2.51 & 3.17 & 0.66 & 5,017 & 72 & 10 & 7 & 4,941 & 136 & 23 & 6 & -76 & 64 & 13 & -1 \\
\hline Mukdahan & 2,139 & 6.76 & 7.70 & 0.94 & 1,761 & 254 & 92 & 32 & 1,679 & 279 & 100 & 81 & -82 & 25 & 8 & 49 \\
\hline Nakhon Nayok & 1,180 & 12.75 & 18.35 & 5.60 & 525 & 544 & 57 & 54 & 99 & 790 & 219 & 72 & -426 & 246 & 162 & 18 \\
\hline Nakhon Pathom & 1,590 & 15.02 & 16.36 & 1.35 & 336 & 1,027 & 221 & 6 & 129 & 1,224 & 235 & 2 & -207 & 197 & 14 & -4 \\
\hline Nakhon Phanom & 4,048 & 7.84 & 8.24 & 0.40 & 3,077 & 654 & 206 & 111 & 2,990 & 738 & 220 & 100 & -87 & 84 & 14 & -11 \\
\hline Nakhon Ratchasima & 13,542 & 4.49 & 5.99 & 1.50 & 12,411 & 807 & 225 & 99 & 11,498 & 1,530 & 328 & 186 & -913 & 723 & 103 & 87 \\
\hline Nakhon Sawan & 7,465 & 8.49 & 11.41 & 2.92 & 5,272 & 1,872 & 245 & 76 & 3,530 & 3,553 & 300 & 82 & $-1,742$ & 1,681 & 55 & 6 \\
\hline Nakhon Si Thammarat & 7,118 & 34.06 & 36.07 & 2.02 & 508 & 1,521 & 1,204 & 3,885 & 283 & 1,227 & 1,236 & 4,372 & -225 & -294 & 32 & 487 \\
\hline Nan & 4,486 & 40.45 & 35.73 & -4.72 & 252 & 437 & 482 & 3,315 & 304 & 854 & 835 & 2,493 & 52 & 417 & 353 & -822 \\
\hline Narathiwat & 3,145 & 45.52 & 47.43 & 1.91 & 26 & 283 & 321 & 2,515 & 15 & 189 & 319 & 2,622 & -11 & -94 & -2 & 107 \\
\hline Nong Bua Lam Phu & 3,559 & 6.46 & 8.78 & 2.33 & 3,016 & 345 & 140 & 58 & 2,723 & 491 & 214 & 131 & -293 & 146 & 74 & 73 \\
\hline Nong Khai & 2,391 & 8.83 & 13.87 & 5.04 & 1,841 & 334 & 86 & 130 & 1,168 & 787 & 251 & 185 & -673 & 453 & 165 & 55 \\
\hline Nonthaburi & 234 & 16.18 & 17.20 & 1.02 & 6 & 201 & 27 & 0 & 5 & 200 & 29 & 0 & -1 & -1 & 2 & 0 \\
\hline Pathum Thani & 797 & 15.55 & 17.86 & 2.31 & 74 & 637 & 86 & 0 & 7 & 650 & 140 & 0 & -67 & 13 & 54 & 0 \\
\hline Pattani & 1,535 & 25.54 & 30.06 & 4.53 & 88 & 673 & 305 & 469 & 31 & 446 & 405 & 653 & -57 & -227 & 100 & 184 \\
\hline Phangnga & 1,725 & 48.18 & 43.68 & -4.50 & 26 & 126 & 179 & 1,394 & 18 & 116 & 242 & 1,349 & -8 & -10 & 63 & -45 \\
\hline Phatthalung & 2,864 & 23.59 & 26.47 & 2.88 & 625 & 992 & 477 & 770 & 385 & 893 & 650 & 936 & -240 & -99 & 173 & 166 \\
\hline Phayao & 2,456 & 22.67 & 20.05 & -2.62 & 1,076 & 413 & 199 & 768 & 1,110 & 485 & 248 & 613 & 34 & 72 & 49 & -155 \\
\hline Phetchabun & 6,090 & 10.50 & 14.75 & 4.25 & 4,365 & 866 & 401 & 458 & 2,927 & 1,974 & 530 & 659 & $-1,438$ & 1,108 & 129 & 201 \\
\hline Phetchaburi & 1,512 & 13.28 & 13.96 & 0.68 & 783 & 489 & 116 & 124 & 559 & 707 & 167 & 79 & -224 & 218 & 51 & -45 \\
\hline Phichit & 4,034 & 8.93 & 10.01 & 1.08 & 2,606 & 1,356 & 69 & 3 & 2,046 & 1,952 & 34 & 2 & -560 & 596 & -35 & -1 \\
\hline Phitsanulok & 4,961 & 16.92 & 16.85 & -0.07 & 2,141 & 1,744 & 331 & 745 & 1,890 & 2,104 & 255 & 712 & -251 & 360 & -76 & -33 \\
\hline Phra Nakhon Si Ayuttha & 1,863 & 13.03 & 14.77 & 1.73 & 571 & 1,162 & 130 & 0 & 129 & 1,660 & 74 & 0 & -442 & 498 & -56 & 0 \\
\hline Phrae & 1,346 & 31.32 & 29.29 & -2.03 & 379 & 212 & 125 & 630 & 354 & 268 & 133 & 591 & -25 & 56 & 8 & -39 \\
\hline Phuket & 287 & 31.15 & 30.48 & -0.67 & 42 & 68 & 43 & 134 & 45 & 60 & 55 & 127 & 3 & -8 & 12 & -7 \\
\hline Prachin Buri & 2,301 & 8.81 & 14.11 & 5.30 & 1,722 & 413 & 86 & 80 & 793 & 1,233 & 165 & 110 & -929 & 820 & 79 & 30 \\
\hline Prachuap Khiri Khan & 2,419 & 19.38 & 19.00 & -0.39 & 685 & 712 & 660 & 362 & 864 & 588 & 506 & 461 & 179 & -124 & -154 & 99 \\
\hline Ranong & 775 & 46.08 & 44.95 & -1.13 & 1 & 29 & 105 & 640 & 4 & 46 & 100 & 625 & 3 & 17 & -5 & -15 \\
\hline
\end{tabular}




\begin{tabular}{|c|c|c|c|c|c|c|c|c|c|c|c|c|c|c|c|c|}
\hline \multicolumn{2}{|c|}{ Thailand } & \multirow{3}{*}{\multicolumn{3}{|c|}{\begin{tabular}{|c|} 
Average Tree Cover by Province \\
Mean Tree Cover Percent by Province \\
$(\%)$
\end{tabular}}} & \multicolumn{12}{|c|}{ Areal Extent of Tree Cover Class $\left(\mathrm{km}^{2}\right)$} \\
\hline \multirow{3}{*}{ Province } & \multirow{3}{*}{ Area_km } & & & & \multicolumn{4}{|c|}{ Year 2000} & \multirow{2}{*}{\multicolumn{4}{|c|}{$\begin{array}{c}\text { Year } 2010 \\
\text { Area by Tree Cover Class }\left(\mathrm{km}^{2}\right)\end{array}$}} & \multicolumn{4}{|c|}{ Change (2000 to 2010) } \\
\hline & & & & & & ea by Tree $\mathrm{C}$ & ver Class ( & & & & & & & by Tree C & ver Class & \\
\hline & & 2000 & 2010 & Change & $<10 \%$ & $10-20 \%$ & $20-30 \%$ & $>\mathbf{3 0 \%}$ & $<10 \%$ & $10-20 \%$ & $20-30 \%$ & $>30 \%$ & $<10 \%$ & $10-20 \%$ & $20-30 \%$ & $>30 \%$ \\
\hline Ratchaburi & 2,487 & 13.21 & 15.10 & 1.90 & 1,230 & 852 & 209 & 196 & 806 & 1,166 & 376 & 139 & -424 & 314 & 167 & -57 \\
\hline Rayong & 2,439 & 18.22 & 18.03 & -0.19 & 657 & 967 & 516 & 299 & 638 & 953 & 558 & 290 & -19 & -14 & 42 & -9 \\
\hline Roi Et & 7,195 & 2.94 & 4.10 & 1.16 & 6,972 & 162 & 40 & 21 & 6,829 & 286 & 58 & 22 & -143 & 124 & 18 & 1 \\
\hline Sa Kaeo & 3,120 & 6.18 & 9.84 & 3.66 & 2,767 & 242 & 62 & 49 & 2,031 & 884 & 112 & 93 & -736 & 642 & 50 & 44 \\
\hline Sakon Nakhon & 7,207 & 5.72 & 8.39 & 2.67 & 6,335 & 703 & 125 & 44 & 5,301 & 1,544 & 230 & 132 & $-1,034$ & 841 & 105 & 88 \\
\hline Samut Prakan & 239 & 10.36 & 14.27 & 3.92 & 118 & 113 & 8 & 0 & 86 & 113 & 39 & 1 & -32 & 0 & 31 & 1 \\
\hline Samut Sakhon & 386 & 17.56 & 15.85 & -1.72 & 146 & 50 & 155 & 35 & 100 & 190 & 96 & 0 & -46 & 140 & -59 & -35 \\
\hline Samut Songkhram & 279 & 29.11 & 25.61 & -3.50 & 32 & 66 & 46 & 135 & 28 & 66 & 84 & 101 & -4 & 0 & 38 & -34 \\
\hline Saraburi & 1,379 & 9.85 & 14.48 & 4.63 & 912 & 366 & 74 & 27 & 409 & 793 & 123 & 54 & -503 & 427 & 49 & 27 \\
\hline Satun & 1,443 & 39.87 & 37.27 & -2.60 & 39 & 184 & 235 & 985 & 18 & 170 & 325 & 930 & -21 & -14 & 90 & -55 \\
\hline Si Sa Ket & 5,460 & 4.66 & 5.99 & 1.33 & 5,107 & 243 & 41 & 69 & 4,902 & 334 & 84 & 140 & -205 & 91 & 43 & 71 \\
\hline Sing Buri & 705 & 12.86 & 15.89 & 3.04 & 211 & 480 & 14 & 0 & 26 & 629 & 50 & 0 & -185 & 149 & 36 & 0 \\
\hline Songkhla & 5,579 & 34.28 & 33.79 & -0.49 & 510 & 1,136 & 820 & 3,113 & 461 & 1,037 & 851 & 3,230 & -49 & -99 & 31 & 117 \\
\hline Sukhothai & 3,856 & 14.32 & 14.86 & 0.55 & 2,327 & 789 & 212 & 528 & 2,123 & 995 & 213 & 525 & -204 & 206 & 1 & -3 \\
\hline Suphan Buri & 4,393 & 13.32 & 15.50 & 2.18 & 1,549 & 2,351 & 335 & 158 & 919 & 2,869 & 423 & 182 & -630 & 518 & 88 & 24 \\
\hline Surat Thani & 8,581 & 39.60 & 44.09 & 4.49 & 62 & 763 & 1,627 & 6,129 & 37 & 441 & 887 & 7,216 & -25 & -322 & -740 & 1,087 \\
\hline Surin & 6,518 & 4.78 & 5.78 & 1.01 & 6,167 & 249 & 30 & 72 & 6,064 & 303 & 67 & 84 & -103 & 54 & 37 & 12 \\
\hline Tak & 3,197 & 33.91 & 30.62 & -3.29 & 712 & 488 & 365 & 1,632 & 743 & 596 & 446 & 1,412 & 31 & 108 & 81 & -220 \\
\hline Trang & 3,188 & 38.91 & 35.42 & -3.49 & 6 & 239 & 725 & 2,218 & 4 & 289 & 996 & 1,899 & -2 & 50 & 271 & -319 \\
\hline Trat & 1,194 & 25.63 & 26.07 & 0.44 & 58 & 429 & 377 & 330 & 22 & 357 & 513 & 302 & -36 & -72 & 136 & -28 \\
\hline Ubon Ratchathani & 9,100 & 5.70 & 6.53 & 0.83 & 8,043 & 569 & 205 & 283 & 7,636 & 972 & 250 & 242 & -407 & 403 & 45 & -41 \\
\hline Udon Thani & 8,939 & 5.57 & 8.05 & 2.49 & 7,877 & 647 & 218 & 197 & 6,901 & 1,278 & 395 & 365 & -976 & 631 & 177 & 168 \\
\hline Uthai Thani & 2,557 & 14.81 & 18.20 & 3.39 & 1,368 & 759 & 111 & 319 & 970 & 912 & 277 & 398 & -398 & 153 & 166 & 79 \\
\hline Uttaradit & 2,549 & 26.32 & 23.85 & -2.47 & 957 & 482 & 165 & 945 & 882 & 594 & 228 & 845 & -75 & 112 & 63 & -100 \\
\hline Yala & 2,533 & 49.53 & 51.49 & 1.97 & 4 & 92 & 162 & 2,275 & 4 & 73 & 117 & 2,339 & 0 & -19 & -45 & 64 \\
\hline Yasothon & 3,545 & 4.36 & 5.08 & 0.71 & 3,324 & 180 & 25 & 16 & 3,206 & 270 & 48 & 21 & -118 & 90 & 23 & 5 \\
\hline Thailand - All & 275,151 & 15.86 & 17.31 & 1.45 & 156,944 & 46,020 & 19,838 & 52,350 & 132,182 & 64,781 & 23,882 & 54,307 & $-24,762$ & 18,761 & 4,044 & 1,957 \\
\hline
\end{tabular}




\begin{tabular}{|c|c|c|c|c|c|c|c|c|c|c|c|c|c|c|c|c|}
\hline \multicolumn{2}{|l|}{ Thailand } & \multirow{3}{*}{\multicolumn{3}{|c|}{$\begin{array}{c}\text { Average Tree Cover by Province } \\
\text { Percent Tree Cover } \\
(\%)\end{array}$}} & \multicolumn{12}{|c|}{ Areal Extent of Tree Cover Class as a Percent of Total Agricultural Area (\%) } \\
\hline \multirow{3}{*}{ Province } & \multirow{3}{*}{ Area_km } & & & & \multicolumn{4}{|c|}{$\begin{array}{r}\text { Year } 2000 \\
\end{array}$} & \multirow{2}{*}{\multicolumn{4}{|c|}{$\begin{array}{c}\text { Year } 2010 \\
\text { Percent of Total Agrciultural Area }(\%) \\
\end{array}$}} & \multirow{2}{*}{\multicolumn{4}{|c|}{$\begin{array}{c}\text { Change }(2000 \text { to } 2010) \\
\text { Percent of Total Agrciultural Area ( \%) }\end{array}$}} \\
\hline & & & & & Perc & ti of Total A & iultural $\mathrm{Ar}$ & \%) & & & & & & & & \\
\hline & & 2000 & 2010 & Change & $<10 \%$ & $10-20 \%$ & $20-30 \%$ & $>30 \%$ & $<10 \%$ & $10-20 \%$ & $20-30 \%$ & $>30 \%$ & $<10 \%$ & $10-20 \%$ & & $>30 \%$ \\
\hline Amnat Charoen & 2,468 & 6.50 & 4.94 & -1.56 & 85.21 & 6.48 & 3.85 & 4.46 & 88.45 & 7.82 & 2.47 & 1.26 & 3.24 & 1.34 & -1.38 & -3.20 \\
\hline Ang Thong & 815 & 14.73 & 16.59 & 1.86 & 10.06 & 85.28 & 4.66 & 0.00 & 3.07 & 89.57 & 7.36 & 0.00 & -6.99 & 4.29 & 2.70 & 0.00 \\
\hline Bangkok Metropolis & 211 & 12.08 & 16.64 & 4.56 & 31.28 & 67.77 & 0.95 & 0.00 & 15.64 & 65.88 & 18.48 & 0.00 & -15.64 & -1.90 & 17.54 & 0.00 \\
\hline Bueng Kan & 3,068 & 7.35 & 15.93 & 8.59 & 83.54 & 12.29 & 2.57 & 1.60 & 34.35 & 39.70 & 17.99 & 7.95 & -49.19 & 27.41 & 15.42 & 6.36 \\
\hline Buri Ram & 7,114 & 4.19 & 6.21 & 2.01 & 95.22 & 3.74 & 0.67 & 0.37 & 89.64 & 7.51 & 1.90 & 0.96 & -5.58 & 3.77 & 1.22 & 0.59 \\
\hline Chachoengsao & 2,873 & 8.54 & 15.45 & 6.91 & 73.62 & 23.11 & 2.37 & 0.90 & 22.00 & 62.34 & 11.66 & 4.00 & -51.62 & 39.23 & 9.29 & 3.10 \\
\hline Chai Nat & 2,052 & 11.60 & 13.50 & 1.90 & 43.67 & 50.34 & 5.56 & 0.44 & 31.77 & 59.50 & 8.38 & 0.34 & -11.89 & 9.16 & 2.83 & -0.10 \\
\hline Chaiyaphum & 7,352 & 5.99 & 9.21 & 3.22 & 86.26 & 8.00 & 3.31 & 2.43 & 72.97 & 18.55 & 3.88 & 4.60 & -13.29 & 10.56 & 0.57 & 2.16 \\
\hline Chanthaburi & 2,331 & 22.61 & 20.96 & -1.64 & 13.77 & 33.89 & 30.89 & 21.45 & 8.28 & 48.61 & 29.39 & 13.73 & -5.49 & 14.71 & -1.50 & -7.72 \\
\hline Chiang Mai & 3,756 & 32.72 & 31.41 & -1.31 & 12.75 & 18.77 & 17.73 & 50.75 & 8.17 & 23.38 & 23.16 & 45.29 & -4.58 & 4.61 & 5.43 & -5.46 \\
\hline Chiang Rai & 4,944 & 27.32 & 24.22 & -3.10 & 33.58 & 15.88 & 9.79 & 40.76 & 33.76 & 21.52 & 11.65 & 33.07 & 0.18 & 5.64 & 1.86 & -7.69 \\
\hline Chon Buri & 2,599 & 10.91 & 13.95 & 3.05 & 68.26 & 21.85 & 4.66 & 5.23 & 44.48 & 35.48 & 13.04 & 7.00 & -23.78 & 13.62 & 8.39 & 1.77 \\
\hline Chumphon & 3,693 & 40.06 & 41.24 & 1.18 & 0.38 & 6.72 & 21.15 & 71.76 & 0.14 & 3.82 & 15.76 & 80.29 & -0.24 & -2.90 & -5.39 & 8.53 \\
\hline Kalasin & 5,631 & 3.32 & 4.84 & 1.51 & 95.56 & 2.95 & 0.99 & 0.50 & 90.68 & 6.68 & 1.55 & 1.10 & -4.88 & 3.73 & 0.55 & 0.60 \\
\hline Kamphaeng Phet & 5,210 & 11.85 & 12.17 & 0.32 & 53.86 & 39.44 & 2.59 & 4.11 & 49.23 & 43.84 & 2.71 & 4.22 & -4.63 & 4.40 & 0.12 & 0.12 \\
\hline Kanchanaburi & 5,692 & 19.10 & 20.22 & 1.12 & 54.06 & 15.23 & 6.47 & 24.24 & 43.75 & 21.17 & 9.70 & 25.39 & -10.31 & 5.94 & 3.23 & 1.14 \\
\hline Khon Kaen & 8,122 & 3.95 & 5.15 & 1.20 & 93.43 & 3.15 & 1.66 & 1.76 & 89.71 & 5.58 & 1.86 & 2.86 & -3.72 & 2.43 & 0.20 & 1.10 \\
\hline Krabi & 3,501 & 42.35 & 46.89 & 4.53 & 0.06 & 3.77 & 15.97 & 80.21 & 0.00 & 1.31 & 6.74 & 91.95 & -0.06 & -2.46 & -9.23 & 11.74 \\
\hline Lampang & 2,202 & 29.08 & 28.20 & -0.88 & 22.75 & 23.84 & 15.35 & 38.06 & 20.12 & 26.57 & 16.12 & 37.19 & -2.63 & 2.72 & 0.77 & -0.86 \\
\hline Lamphun & 987 & 13.65 & 16.40 & 2.74 & 50.66 & 29.18 & 11.85 & 8.31 & 29.48 & 45.49 & 17.73 & 7.29 & -21.18 & 16.31 & 5.88 & -1.01 \\
\hline Loei & 5,050 & 18.62 & 20.68 & 2.05 & 30.42 & 34.63 & 19.37 & 15.58 & 21.52 & 36.95 & 21.66 & 19.86 & -8.89 & 2.32 & 2.30 & 4.28 \\
\hline Lop Buri & 4,816 & 7.71 & 13.19 & 5.47 & 75.50 & 22.36 & 1.95 & 0.19 & 32.43 & 58.45 & 7.45 & 1.66 & -43.07 & 36.09 & 5.50 & 1.47 \\
\hline Mae Hong Son & 1,626 & 48.10 & 44.32 & -3.78 & 0.25 & 2.40 & 4.24 & 93.11 & 1.72 & 4.86 & 11.19 & 82.23 & 1.48 & 2.46 & 6.95 & -10.89 \\
\hline Maha Sarakham & 5,106 & 2.51 & 3.17 & 0.66 & 98.26 & 1.41 & 0.20 & 0.14 & 96.77 & 2.66 & 0.45 & 0.12 & -1.49 & 1.25 & 0.25 & -0.02 \\
\hline Mukdahan & 2,139 & 6.76 & 7.70 & 0.94 & 82.33 & 11.87 & 4.30 & 1.50 & 78.50 & 13.04 & 4.68 & 3.79 & -3.83 & 1.17 & 0.37 & 2.29 \\
\hline Nakhon Nayok & 1,180 & 12.75 & 18.35 & 5.60 & 44.49 & 46.10 & 4.83 & 4.58 & 8.39 & 66.95 & 18.56 & 6.10 & -36.10 & 20.85 & 13.73 & 1.53 \\
\hline Nakhon Pathom & 1,590 & 15.02 & 16.36 & 1.35 & 21.13 & 64.59 & 13.90 & 0.38 & 8.11 & 76.98 & 14.78 & 0.13 & -13.02 & 12.39 & 0.88 & -0.25 \\
\hline Nakhon Phanom & 4,048 & 7.84 & 8.24 & 0.40 & 76.01 & 16.16 & 5.09 & 2.74 & 73.86 & 18.23 & 5.43 & 2.47 & -2.15 & 2.08 & 0.35 & -0.27 \\
\hline Nakhon Ratchasima & 13,542 & 4.49 & 5.99 & 1.50 & 91.65 & 5.96 & 1.66 & 0.73 & 84.91 & 11.30 & 2.42 & 1.37 & -6.74 & 5.34 & 0.76 & 0.64 \\
\hline Nakhon Sawan & 7,465 & 8.49 & 11.41 & 2.92 & 70.62 & 25.08 & 3.28 & 1.02 & 47.29 & 47.60 & 4.02 & 1.10 & -23.34 & 22.52 & 0.74 & 0.08 \\
\hline Nakhon Si Thammarat & 7,118 & 34.06 & 36.07 & 2.02 & 7.14 & 21.37 & 16.91 & 54.58 & 3.98 & 17.24 & 17.36 & 61.42 & -3.16 & -4.13 & 0.45 & 6.84 \\
\hline Nan & 4,486 & 40.45 & 35.73 & -4.72 & 5.62 & 9.74 & 10.74 & 73.90 & 6.78 & 19.04 & 18.61 & 55.57 & 1.16 & 9.30 & 7.87 & $\begin{array}{l}-18.32 \\
-184\end{array}$ \\
\hline Narathiwat & 3,145 & 45.52 & 47.43 & 1.91 & 0.83 & 9.00 & 10.21 & 79.97 & 0.48 & 6.01 & 10.14 & 83.37 & -0.35 & -2.99 & -0.06 & 3.40 \\
\hline Nong Bua Lam Phu & 3,559 & 6.46 & 8.78 & 2.33 & 84.74 & 9.69 & 3.93 & 1.63 & 76.51 & 13.80 & 6.01 & 3.68 & -8.23 & 4.10 & 2.08 & 2.05 \\
\hline Nong Khai & 2,391 & 8.83 & 13.87 & 5.04 & 77.00 & 13.97 & 3.60 & 5.44 & 48.85 & 32.92 & 10.50 & 7.74 & -28.15 & 18.95 & 6.90 & 2.30 \\
\hline Nonthaburi & 234 & 16.18 & 17.20 & 1.02 & 2.56 & 85.90 & 11.54 & 0.00 & 2.14 & 85.47 & 12.39 & 0.00 & -0.43 & -0.43 & 0.85 & 0.00 \\
\hline Pathum Thani & 797 & 15.55 & 17.86 & 2.31 & 9.28 & 79.93 & 10.79 & 0.00 & 0.88 & 81.56 & 17.57 & 0.00 & -8.41 & 1.63 & 6.78 & 0.00 \\
\hline Pattani & 1,535 & 25.54 & 30.06 & 4.53 & 5.73 & 43.84 & 19.87 & 30.55 & 2.02 & 29.06 & 26.38 & 42.54 & -3.71 & -14.79 & 6.51 & 11.99 \\
\hline Phangnga & 1,725 & 48.18 & 43.68 & -4.50 & 1.51 & 7.30 & 10.38 & 80.81 & 1.04 & 6.72 & 14.03 & 78.20 & -0.46 & -0.58 & 3.65 & -2.61 \\
\hline Phatthalung & 2,864 & 23.59 & 26.47 & 2.88 & 21.82 & 34.64 & 16.66 & 26.89 & 13.44 & 31.18 & 22.70 & 32.68 & -8.38 & -3.46 & 6.04 & 5.80 \\
\hline Phayao & 2,456 & 22.67 & 20.05 & -2.62 & 43.81 & 16.82 & 8.10 & 31.27 & 45.20 & 19.75 & 10.10 & 24.96 & 1.38 & 2.93 & 2.00 & -6.31 \\
\hline Phetchabun & 6,090 & 10.50 & 14.75 & 4.25 & 71.68 & 14.22 & 6.58 & 7.52 & 48.06 & 32.41 & 8.70 & 10.82 & -23.61 & 18.19 & 2.12 & 3.30 \\
\hline Phetchaburi & 1,512 & 13.28 & 13.96 & 0.68 & 51.79 & 32.34 & 7.67 & 8.20 & 36.97 & 46.76 & 11.05 & 5.22 & -14.81 & 14.42 & 3.37 & -2.98 \\
\hline Phichit & 4,034 & 8.93 & 10.01 & $\begin{array}{l}.00 \\
1.08\end{array}$ & 64.60 & 33.61 & 1.71 & 0.07 & 50.72 & 48.39 & 0.84 & 0.05 & -13.88 & 14.77 & $\begin{array}{l}-0.87 \\
-0.87\end{array}$ & -0.02 \\
\hline Phitsanulok & 4,961 & 16.92 & 16.85 & -0.07 & 43.16 & 35.15 & 6.67 & 15.02 & 38.10 & 42.41 & 5.14 & 14.35 & -5.06 & 7.26 & -1.53 & -0.67 \\
\hline Phra Nakhon Si Ayuttha & 1,863 & 13.03 & 14.77 & 1.73 & 30.65 & 62.37 & 6.98 & 0.00 & 6.92 & 89.10 & 3.97 & 0.00 & -23.73 & 26.73 & -3.01 & 0.00 \\
\hline Phrae & 1,346 & 31.32 & 29.29 & -2.03 & 28.16 & 15.75 & 9.29 & 46.81 & 26.30 & 19.91 & 9.88 & 43.91 & -1.86 & 4.16 & 0.59 & -2.90 \\
\hline Phuket & 287 & 31.15 & 30.48 & -0.67 & 14.63 & 23.69 & 14.98 & 46.69 & 15.68 & 20.91 & 19.16 & 44.25 & 1.05 & -2.79 & 4.18 & -2.44 \\
\hline Prachin Buri & 2,301 & 8.81 & 14.11 & 5.30 & 74.84 & 17.95 & 3.74 & 3.48 & 34.46 & 53.59 & 7.17 & 4.78 & -40.37 & 35.64 & 3.43 & 1.30 \\
\hline Prachuap Khiri Khan & 2,419 & 19.38 & 19.00 & $\begin{array}{r}0.00 \\
-0.39\end{array}$ & 28.32 & 29.43 & 27.28 & $\begin{array}{r}14.40 \\
14.96\end{array}$ & 35.72 & 24.31 & 20.92 & 19.06 & 7.40 & -5.13 & $\begin{array}{l}.0 .40 \\
-6.37\end{array}$ & 4.09 \\
\hline Ranong & 775 & 46.08 & 44.95 & -1.13 & 0.13 & 3.74 & 13.55 & 82.58 & 0.52 & 5.94 & 12.90 & 80.65 & 0.39 & 2.19 & -0.65 & -1.94 \\
\hline
\end{tabular}




\begin{tabular}{|c|c|c|c|c|c|c|c|c|c|c|c|c|c|c|c|c|}
\hline \multicolumn{2}{|c|}{ Thailand } & \multirow{3}{*}{\multicolumn{3}{|c|}{$\begin{array}{l}\text { Average Tree Cover by Province } \\
\text { Percent Tree Cover } \\
(\%)\end{array}$}} & \multicolumn{12}{|c|}{ Areal Extent of Tree Cover Class as a Percent of Total Agricultural Area (\%) } \\
\hline \multirow{2}{*}{ Province } & \multirow{2}{*}{ Area_km } & & & & \multirow{2}{*}{\multicolumn{4}{|c|}{$\begin{array}{c}\text { Year } 2000 \\
\text { Percent of Total Agrciultural Area (\%) }\end{array}$}} & \multirow{2}{*}{\multicolumn{4}{|c|}{$\begin{array}{c}\text { Year } 2010 \\
\text { Percent of Total Agrciultural Area (\%) }\end{array}$}} & \multirow{2}{*}{\multicolumn{4}{|c|}{$\begin{array}{c}\text { Change (2000 to } 2010) \\
\text { Percent of Total Agrciultural Area (\%) }\end{array}$}} \\
\hline & & & & & $\begin{array}{l}\text { Perc } \\
<0 \%\end{array}$ & & & & & & & & & & & \\
\hline Ratchaburi & 2487 & 1321 & & Cnange & 4946 & $\frac{10-20 \%}{3426}$ & $20-30 \%$ & $>30 \%$ & $<10 \%$ & $10-20 \%$ & $20-30 \%$ & $>30 \%$ & $<10 \%$ & $10-20 \%$ & $20-30 \%$ & $>30 \%$ \\
\hline Rayong & 2,439 & 18.22 & 18.03 & $\begin{array}{r}1.90 \\
-0.19\end{array}$ & 26.94 & $\begin{array}{l}34.26 \\
3965\end{array}$ & 8.40 & 7.88 & 32.41 & 40.88 & 10.12 & 5.59 & -17.05 & 12.63 & 6.71 & -2.29 \\
\hline Roi Et & 7,195 & 2.94 & 4.10 & 1.16 & 96.90 & 2.25 & 0.56 & 0.29 & 94.91 & 3.98 & 0.81 & 0.31 & -1.99 & 1.72 & 0.25 & 0.01 \\
\hline Sa Kaeo & 3.120 & 6.18 & 9.84 & 3.66 & 88.69 & 7.76 & 1.99 & 1.57 & 65.10 & 28.33 & 3.59 & 2.98 & -23.59 & 20.58 & 1.60 & 1.41 \\
\hline Sakon Nakhon & 7,207 & 5.72 & 8.39 & 2.67 & 87.90 & 9.75 & 1.73 & 0.61 & 73.55 & 21.42 & 3.19 & 1.83 & -14.35 & 11.67 & 1.46 & 1.22 \\
\hline Samut Prakan & 239 & 10.36 & 14.27 & 3.92 & 49.37 & 47.28 & 3.35 & 0.00 & 35.98 & 47.28 & 16.32 & 0.42 & -13.39 & 0.00 & 12.97 & 0.42 \\
\hline Samut Sakhon & 386 & 17.56 & 15.85 & -1.72 & 37.82 & 12.95 & 40.16 & 9.07 & 25.91 & 49.22 & 24.87 & 0.00 & -11.92 & 36.27 & -15.29 & -9.07 \\
\hline Samut Songkhram & 279 & 29.11 & 25.61 & -3.50 & 11.47 & 23.66 & 16.49 & 48.39 & 10.04 & 23.66 & 30.11 & 36.20 & -1.43 & 0.00 & 13.62 & -12.19 \\
\hline Saraburi & 1,379 & 9.85 & 14.48 & 4.63 & 66.14 & 26.54 & 5.37 & 1.96 & 29.66 & 57.51 & 8.92 & 3.92 & -36.48 & 30.96 & 3.55 & 1.96 \\
\hline Satun & 1.443 & 39.87 & 37.27 & -2.60 & 2.70 & 12.75 & 16.29 & 68.26 & 1.25 & 11.78 & 22.52 & 64.45 & -1.46 & -0.97 & 6.24 & -3.81 \\
\hline Si Sa Ket & 5,460 & 4.66 & 5.99 & 1.33 & 93.54 & 4.45 & 0.75 & 1.26 & 89.78 & 6.12 & 1.54 & 2.56 & -3.75 & 1.67 & 0.79 & 1.30 \\
\hline Sing Buri & 705 & 12.86 & 15.89 & 3.04 & 29.93 & 68.09 & 1.99 & 0.00 & 3.69 & 89.22 & 7.09 & 0.00 & -26.24 & 21.13 & 5.11 & 0.00 \\
\hline Songkhla & 5,579 & 34.28 & 33.79 & -0.49 & 9.14 & 20.36 & 14.70 & 55.80 & 8.26 & 18.59 & 15.25 & 57.90 & -0.88 & -1.77 & 0.56 & 2.10 \\
\hline Sukhothai & 3,856 & 14.32 & 14.86 & 0.55 & 60.35 & 20.46 & 5.50 & 13.69 & 55.06 & 25.80 & 5.52 & 13.62 & -5.29 & 5.34 & 0.03 & -0.08 \\
\hline Suphan Buri & 4,393 & 13.32 & 15.50 & 2.18 & 35.26 & 53.52 & 7.63 & 3.60 & 20.92 & 65.31 & 9.63 & 4.14 & -14.34 & 11.79 & 2.00 & 0.55 \\
\hline Surat Thani & 8.581 & 39.60 & 44.09 & 4.49 & 0.72 & 8.89 & 18.96 & 71.43 & 0.43 & 5.14 & 10.34 & 84.09 & -0.29 & -3.75 & -8.62 & 12.67 \\
\hline Surin & 6,518 & 4.78 & 5.78 & 1.01 & 94.62 & 3.82 & 0.46 & 1.10 & 93.04 & 4.65 & 1.03 & 1.29 & -1.58 & 0.83 & 0.57 & 0.18 \\
\hline Tak & 3,197 & 33.91 & 30.62 & -3.29 & 22.27 & 15.26 & 11.42 & 51.05 & 23.24 & 18.64 & 13.95 & 44.17 & 0.97 & 3.38 & 2.53 & -6.88 \\
\hline Trang & 3,188 & 38.91 & 35.42 & -3.49 & 0.19 & 7.50 & 22.74 & 69.57 & 0.13 & 9.07 & 31.24 & 59.57 & -0.06 & 1.57 & 8.50 & -10.01 \\
\hline Trat & 1,194 & 25.63 & 26.07 & 0.44 & 4.86 & 35.93 & 31.57 & 27.64 & 1.84 & 29.90 & 42.97 & 25.29 & -3.02 & -6.03 & 11.39 & -2.35 \\
\hline Ubon Ratchathani & 9,100 & 5.70 & 6.53 & 0.83 & 88.39 & 6.25 & 2.25 & 3.11 & 83.91 & 10.68 & 2.75 & 2.66 & -4.47 & 4.43 & 0.49 & -0.45 \\
\hline Udon Thani & 8,939 & 5.57 & 8.05 & 2.49 & 88.12 & 7.24 & 2.44 & 2.20 & 77.20 & 14.30 & 4.42 & 4.08 & -10.92 & 7.06 & 1.98 & 1.88 \\
\hline Uthai Thani & 2,557 & 14.81 & 18.20 & 3.39 & 53.50 & 29.68 & 4.34 & 12.48 & 37.94 & 35.67 & 10.83 & 15.57 & -15.57 & 5.98 & 6.49 & 3.09 \\
\hline Uttaradit & 2,549 & 26.32 & 23.85 & -2.47 & 37.54 & 18.91 & 6.47 & 37.07 & 34.60 & 23.30 & 8.94 & 33.15 & -2.94 & 4.39 & 2.47 & -3.92 \\
\hline & 2,533 & 49.53 & 51.49 & 1.97 & 0.16 & 3.63 & 6.40 & 89.82 & 0.16 & 2.88 & 4.62 & 92.34 & 0.00 & -0.75 & -1.78 & 2.53 \\
\hline Yasothon & 3,545 & 4.36 & 5.08 & 0.71 & 93.77 & 5.08 & 0.71 & 0.45 & 90.44 & 7.62 & 1.35 & 0.59 & -3.33 & 2.54 & 0.65 & 0.14 \\
\hline Thailand - All & 275.151 & 15.86 & 17.31 & 1.45 & 57.04 & 16.73 & 721 & 1903 & 48.04 & 2354 & 8.68 & 1974 & -900 & 6.82 & 1.47 & 0.71 \\
\hline
\end{tabular}


Asia Pacific Region : Tree Cover on Agricultural Land (2010)

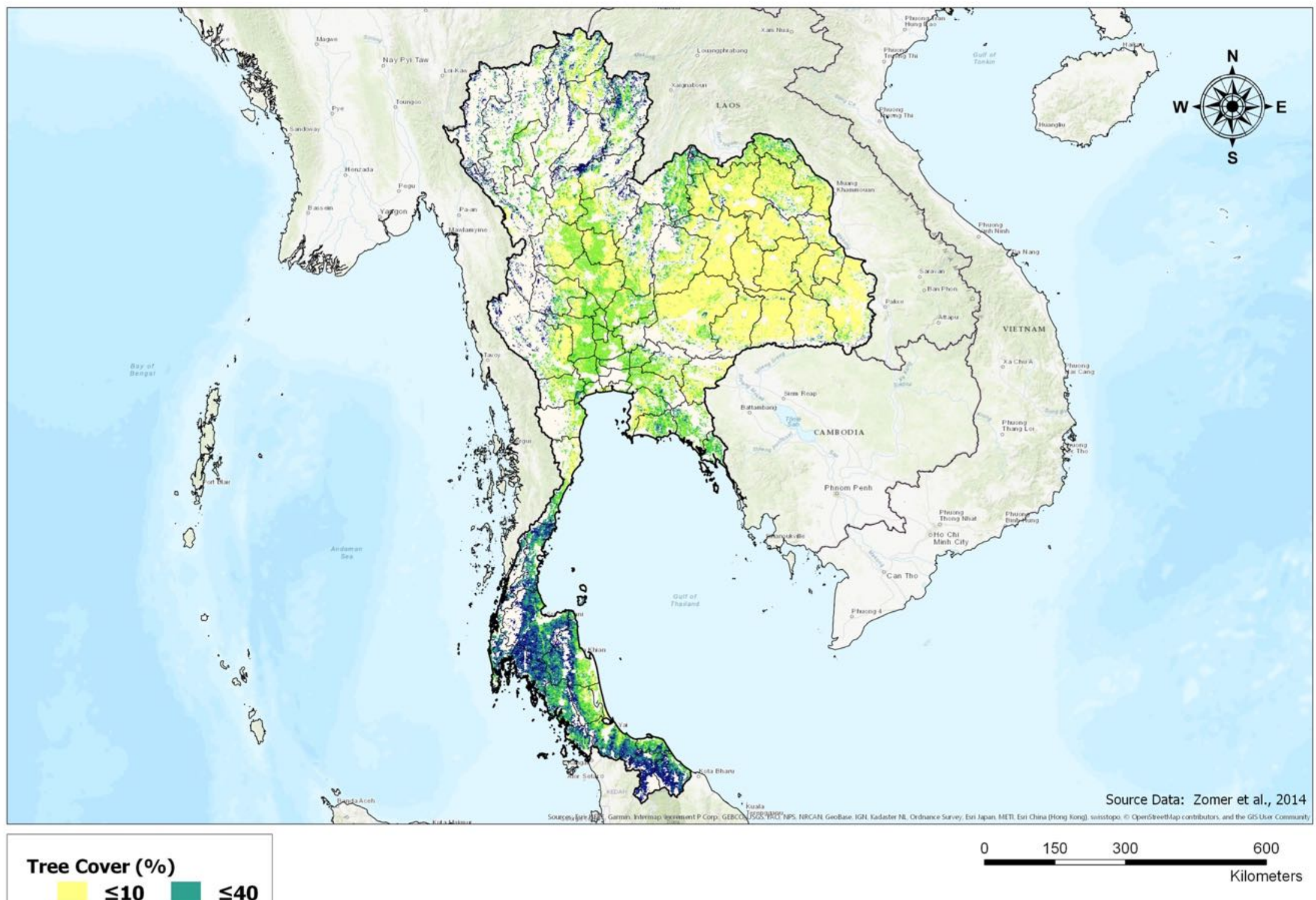

Thailand 
Asia Pacific Region : Change in Tree Cover on Agricultural Land (2000 - 2010)

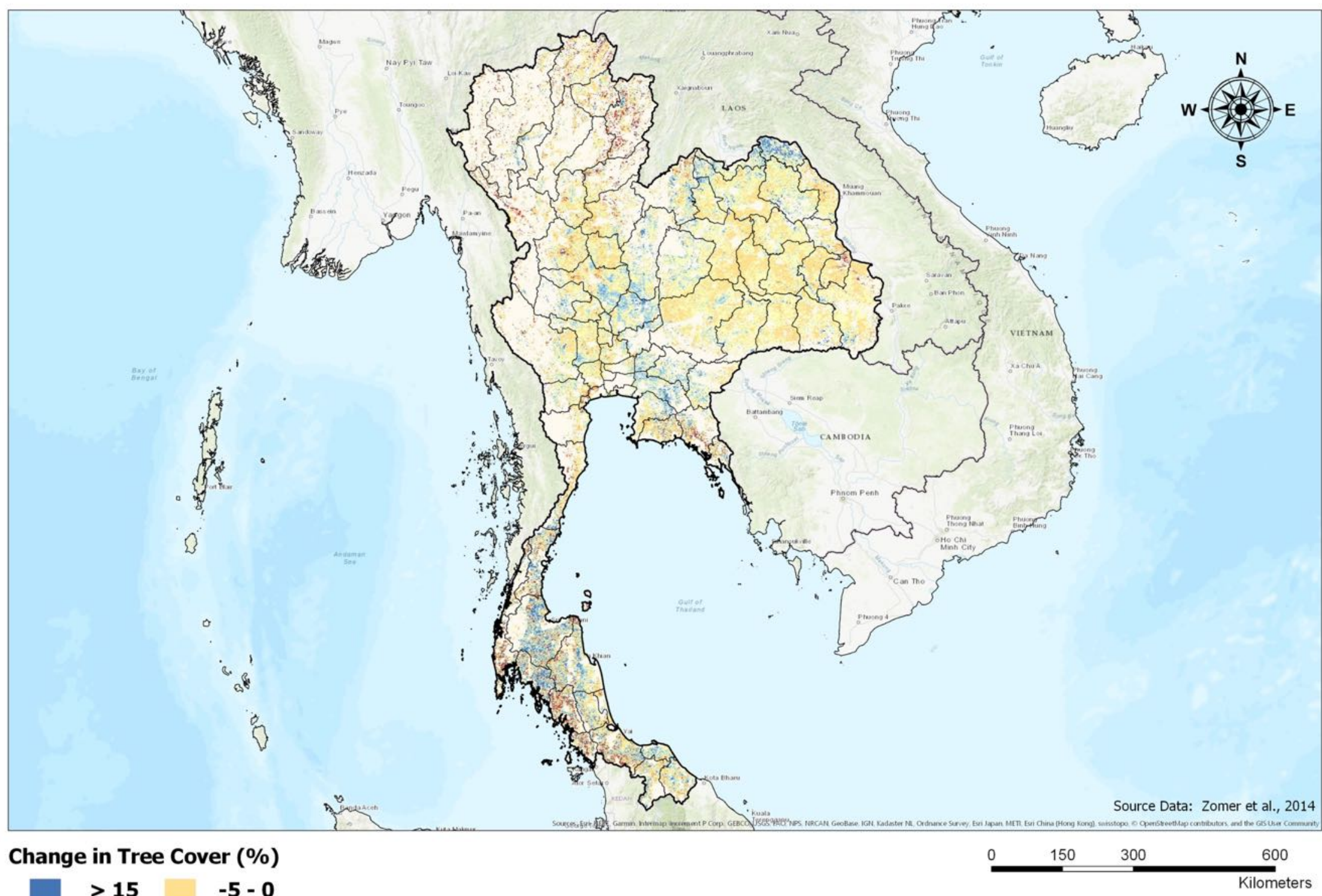

$10-15 \quad-10--5$

$5-10-15--10$

Thailand

$0-5 \square \leq-15$ 
Appendix 24: Timor Leste

Average Percent Tree Cover by Province, and Areal Extent of Tree Cover Classes $\left(\mathrm{km}^{2}\right)$

\begin{tabular}{|c|c|c|c|c|c|c|c|c|c|c|c|c|c|c|c|c|}
\hline \multicolumn{2}{|c|}{ Timor Leste } & \multicolumn{3}{|c|}{ Average Tree Cover by Province } & \multicolumn{12}{|c|}{ Areal Extent of Tree Cover Class $\left(\mathrm{km}^{2}\right)$} \\
\hline \multirow{3}{*}{ Province } & \multirow{3}{*}{ Area_km } & \multirow{2}{*}{\multicolumn{3}{|c|}{$\begin{array}{c}\text { Mean Tree Cover Percent by Province } \\
(\%)\end{array}$}} & \multirow{2}{*}{\multicolumn{4}{|c|}{$\begin{array}{c}\text { Year } 2000 \\
\text { Area by Tree Cover Class }\left(\mathrm{km}^{2}\right)\end{array}$}} & \multirow{2}{*}{\multicolumn{4}{|c|}{$\begin{array}{c}\text { Year } 2010 \\
\text { Area by Tree Cover Class }\left(\mathrm{km}^{2}\right)\end{array}$}} & \multirow{2}{*}{\multicolumn{4}{|c|}{$\begin{array}{c}\text { Change (2000 to 2010) } \\
\text { Area by Tree Cover Class }\left(\mathrm{km}^{2}\right)\end{array}$}} \\
\hline & & & & & & & & & & & & & & & & \\
\hline & & 2000 & 2010 & Change & $<10 \%$ & $10-20 \%$ & $20-30 \%$ & $>30 \%$ & $<10 \%$ & $10-20 \%$ & $20-30 \%$ & $>30 \%$ & $<10 \%$ & $10-20 \%$ & $20-30 \%$ & $>30 \%$ \\
\hline Aileu & 172 & 21.90 & 25.35 & 3.45 & 31 & 55 & $\frac{43}{3}$ & 43 & 11 & 52 & 55 & 54 & -20 & -3 & 12 & 11 \\
\hline Ainaro & 440 & 27.57 & 30.04 & 2.47 & 41 & 135 & 94 & 170 & 6 & 87 & 152 & 195 & -35 & -48 & 58 & 25 \\
\hline Ambeno & 255 & 15.90 & 16.04 & 0.14 & 80 & 120 & 39 & 16 & 78 & 113 & 50 & 14 & -2 & -7 & 11 & -2 \\
\hline Baucau & 687 & 18.22 & 23.91 & 5.70 & 234 & 205 & 131 & 117 & 131 & 178 & 172 & 206 & -103 & -27 & 41 & 89 \\
\hline Bobonaro & 440 & 25.75 & 28.99 & 3.24 & 48 & 165 & 74 & 153 & 57 & 96 & 102 & 185 & 9 & $\begin{array}{l}-69 \\
-69 \\
\text { a }\end{array}$ & 28 & 32 \\
\hline Covalima & 734 & 34.19 & 35.62 & 1.43 & 37 & 134 & 146 & 417 & 5 & 114 & 180 & 435 & -32 & -20 & 34 & 18 \\
\hline Dili & 52 & 19.00 & 23.17 & 4.17 & 20 & 15 & 6 & 11 & 13 & 14 & 10 & 15 & -7 & -1 & 4 & 4 \\
\hline Ermera & 327 & 31.93 & 34.13 & 2.20 & 21 & 64 & 72 & 170 & 8 & 51 & 78 & 190 & -13 & -13 & 6 & 20 \\
\hline Lautem & 842 & 35.98 & 39.69 & 3.72 & 54 & 162 & 143 & 483 & 22 & 144 & 105 & 571 & -32 & -18 & -38 & 88 \\
\hline Liguica & 267 & 42.53 & 43.75 & 1.22 & 23 & 21 & 26 & 197 & 7 & 29 & 29 & 202 & -16 & 8 & 3 & 5 \\
\hline Manatuto & 486 & 30.42 & 34.43 & 4.01 & 90 & 57 & 71 & 268 & 87 & 46 & 59 & 294 & -3 & -11 & -12 & 26 \\
\hline Manufahi & 687 & 36.41 & 37.91 & 1.50 & 3 & 99 & 155 & 430 & 0 & 60 & 165 & 462 & -3 & -39 & 10 & 32 \\
\hline Viqueque & 1,024 & 32.94 & 37.67 & 4.74 & 13 & 229 & 260 & 522 & 2 & 115 & 239 & 668 & -11 & -114 & -21 & 146 \\
\hline Timor Leste - All & 6,411 & 30.49 & 33.66 & 3.17 & 696 & 1,459 & 1,259 & 2,997 & 427 & 1,099 & 1,394 & 3,491 & -269 & -360 & 135 & 494 \\
\hline
\end{tabular}

Average Percent Tree Cover by Province, and Areal Extent of Tree Cover Classes as a Percent of the Total Agricultural Area (\%)

\begin{tabular}{|c|c|c|c|c|c|c|c|c|c|c|c|c|c|c|c|c|}
\hline \multicolumn{2}{|c|}{ Timor Leste } & \multirow{3}{*}{\multicolumn{3}{|c|}{$\begin{array}{c}\text { Average Tree Cover by Province } \\
\text { Percent Tree Cover } \\
(\%)\end{array}$}} & \multicolumn{12}{|c|}{ Areal Extent of Tree Cover Class as a Percent of Total Agricultural Area (\%) } \\
\hline \multirow{3}{*}{ Province } & \multirow{3}{*}{ Area_km } & & & & \multirow{2}{*}{\multicolumn{4}{|c|}{$\begin{array}{c}\text { Year } 2000 \\
\text { Percent of Total Aarciultural Area (\%) }\end{array}$}} & \multirow{2}{*}{\multicolumn{4}{|c|}{$\begin{array}{c}\text { Year } 2010 \\
\text { Percent of Total Agrciultural Area (\%) }\end{array}$}} & \multirow{2}{*}{\multicolumn{4}{|c|}{$\begin{array}{c}\text { Change }(2000 \text { to } 2010) \\
\end{array}$}} \\
\hline & & & & & & & & & & & & & Perc & & & \\
\hline & & 2000 & 2010 & Change & $<10 \%$ & $10-20 \%$ & $20-30 \%$ & $>30 \%$ & $<10 \%$ & $10-20 \%$ & $20-30 \%$ & $>30 \%$ & $<10 \%$ & $10-20 \%$ & $20-30 \%$ & $>30 \%$ \\
\hline Aileu & 172 & 21.90 & 25.35 & 3.45 & 18.02 & 31.98 & 25.00 & 25.00 & 6.40 & 30.23 & 31.98 & 31.40 & -11.63 & -1.74 & 6.98 & 6.40 \\
\hline Ainaro & 440 & 27.57 & 30.04 & 2.47 & 9.32 & 30.68 & 21.36 & 38.64 & 1.36 & 19.77 & 34.55 & 44.32 & -7.95 & -10.91 & 13.18 & 5.68 \\
\hline Ambeno & 255 & 15.90 & 16.04 & 0.14 & 31.37 & 47.06 & 15.29 & 6.27 & 30.59 & 44.31 & 19.61 & 5.49 & -0.78 & -2.75 & 4.31 & -0.78 \\
\hline Baucau & 687 & 18.22 & 23.91 & 5.70 & 34.06 & 29.84 & 19.07 & 17.03 & 19.07 & 25.91 & 25.04 & 29.99 & -14.99 & -3.93 & 5.97 & 12.95 \\
\hline Bobonaro & 440 & 25.75 & 28.99 & 3.24 & 10.91 & 37.50 & 16.82 & 34.77 & 12.95 & 21.82 & 23.18 & 42.05 & 2.05 & -15.68 & 6.36 & 7.27 \\
\hline Covalima & 734 & 34.19 & 35.62 & 1.43 & 5.04 & 18.26 & 19.89 & 56.81 & 0.68 & 15.53 & 24.52 & 59.26 & -4.36 & -2.72 & 4.63 & 2.45 \\
\hline Dili & 52 & 19.00 & 23.17 & 4.17 & 38.46 & 28.85 & 11.54 & 21.15 & 25.00 & 26.92 & 19.23 & 28.85 & -13.46 & -1.92 & 7.69 & 7.69 \\
\hline Ermera & 327 & 31.93 & 34.13 & 2.20 & 6.42 & 19.57 & 22.02 & 51.99 & 2.45 & 15.60 & 23.85 & 58.10 & -3.98 & -3.98 & 1.83 & 6.12 \\
\hline Lautem & 842 & 35.98 & 39.69 & 3.72 & 6.41 & 19.24 & 16.98 & 57.36 & 2.61 & 17.10 & 12.47 & 67.82 & -3.80 & -2.14 & -4.51 & 10.45 \\
\hline Liguica & 267 & 42.53 & 43.75 & 1.22 & 8.61 & 7.87 & 9.74 & 73.78 & 2.62 & 10.86 & 10.86 & 75.66 & -5.99 & 3.00 & 1.12 & 1.87 \\
\hline Manatuto & 486 & 30.42 & 34.43 & 4.01 & 18.52 & 11.73 & 14.61 & 55.14 & 17.90 & 9.47 & 12.14 & 60.49 & -0.62 & -2.26 & -2.47 & 5.35 \\
\hline Manufahi & 687 & 36.41 & 37.91 & 1.50 & 0.44 & 14.41 & 22.56 & 62.59 & 0.00 & 8.73 & 24.02 & 67.25 & -0.44 & -5.68 & 1.46 & 4.66 \\
\hline Viqueque & 1,024 & 32.94 & 37.67 & 4.74 & 1.27 & 22.36 & 25.39 & 50.98 & 0.20 & 11.23 & 23.34 & 65.23 & -1.07 & -11.13 & -2.05 & 14.26 \\
\hline Timor Leste - All & 6.411 & 30.49 & 33.66 & 3.17 & 10.86 & 22.76 & 19.64 & 46.75 & 6.66 & 17.14 & 21.74 & 54.45 & -4.20 & -5.62 & 2.11 & 771 \\
\hline
\end{tabular}


Asia Pacific Region : Tree Cover on Agricultural Land (2010)

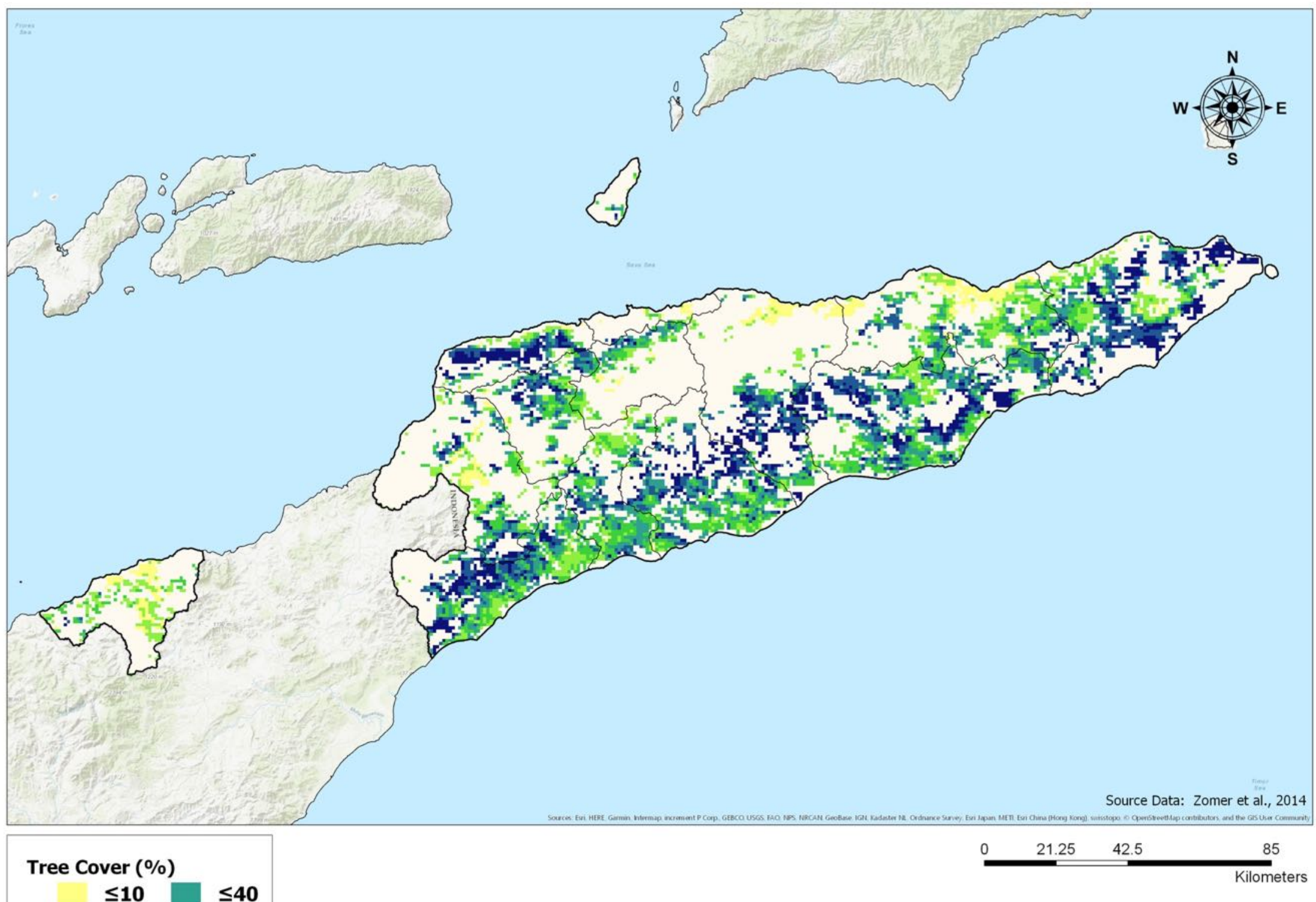

Timor Leste 
Asia Pacific Region : Change in Tree Cover on Agricultural Land (2000 - 2010)

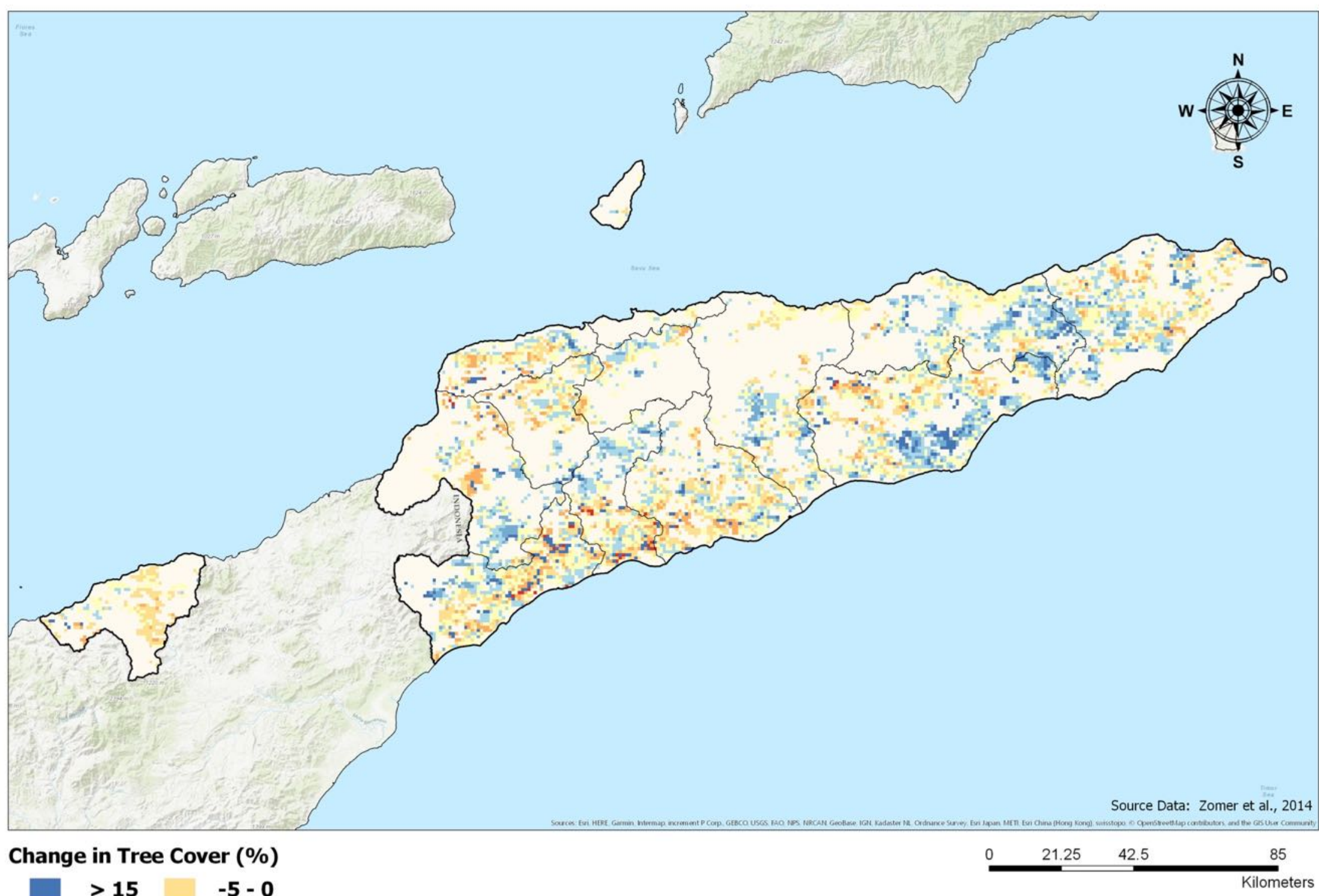

\begin{tabular}{|r|r|}
$>15$ & $-5-0$ \\
$10-15$ & $-10--5$ \\
$5-10$ & $-15--10$ \\
$0-5$ & $\leq-15$
\end{tabular}

Timor Leste 
Average Percent Tree Cover by Province, and Areal Extent of Tree Cover Classes $\left(\mathrm{km}^{2}\right)$

\begin{tabular}{|c|c|c|c|c|c|c|c|c|c|c|c|c|c|c|c|c|}
\hline \multicolumn{2}{|c|}{ Vanuatu } & \multirow{3}{*}{\multicolumn{3}{|c|}{\begin{tabular}{|c|} 
Average Tree Cover by Province \\
Mean Tree Cover Percent by Province \\
$(\%)$
\end{tabular}}} & \multicolumn{12}{|c|}{ Areal Extent of Tree Cover Class $\left(\mathrm{km}^{2}\right)$} \\
\hline \multirow{3}{*}{ Province } & \multirow{3}{*}{ Area_km } & & & & \multicolumn{4}{|c|}{ Year 2000 } & \multirow{2}{*}{\multicolumn{4}{|c|}{$\begin{array}{c}\text { Year } 2010 \\
\text { Area by Tree Cover Class }\left(\mathrm{km}^{2}\right)\end{array}$}} & \multirow{2}{*}{\multicolumn{4}{|c|}{ Change (2000 to 2010) }} \\
\hline & & & & & & by Tree $\mathrm{C}$ & ver Class $(k \mathrm{kr}$ & & & & & & & & & \\
\hline & & 2000 & 2010 & Change & $<10 \%$ & $10-20 \%$ & $20-30 \%$ & $>\mathbf{3 0 \%}$ & $<10 \%$ & $10-20 \%$ & $20-30 \%$ & $>30 \%$ & $<10 \%$ & $10-20 \%$ & $20-30 \%$ & $>30 \%$ \\
\hline Malampa & 1,064 & 66.85 & 70.18 & 3.33 & $\overline{0}$ & 0 & 2 & 1,062 & 0 & 0 & 3 & 1,061 & 0 & 0 & 1 & -1 \\
\hline Penama & 310 & 59.89 & 63.22 & 3.33 & 0 & 4 & 4 & 302 & 0 & 0 & 0 & 310 & 0 & -4 & -4 & 8 \\
\hline Sanma & 2,423 & 63.02 & 69.84 & 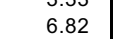 & 0 & 4 & 17 & 2,402 & 0 & 0 & 1 & 2,422 & 0 & -4 & -16 & 20 \\
\hline Shefa & 107 & 69.65 & 72.25 & 2.60 & 0 & 0 & 0 & 107 & 0 & 0 & 0 & 107 & 0 & 0 & 0 & 0 \\
\hline Torba & 80 & 63.76 & 64.93 & 1.16 & 0 & 0 & 0 & 80 & 0 & 0 & 1 & 79 & 0 & 0 & 1 & -1 \\
\hline Vanuatu - All & 3,984 & 63.99 & 69.38 & 5.39 & 0 & 8 & 23 & 3,953 & 0 & 0 & 5 & 3,979 & 0 & -8 & -18 & 26 \\
\hline
\end{tabular}

Average Percent Tree Cover by Province, and Areal Extent of Tree Cover Classes as a Percent of the Total Agricultural Area (\%)

\begin{tabular}{|c|c|c|c|c|c|c|c|c|c|c|c|c|c|c|c|c|}
\hline \multicolumn{2}{|c|}{ Vanuatu } & \multirow{3}{*}{\multicolumn{3}{|c|}{$\begin{array}{c}\text { Average Tree Cover by Province } \\
\text { Percent Tree Cover } \\
(\%)\end{array}$}} & \multicolumn{12}{|c|}{ Class as a Percent of Total Agricultural Area (\%) } \\
\hline \multirow{3}{*}{ Province } & \multirow{3}{*}{ Area_km } & & & & \multirow{2}{*}{\multicolumn{4}{|c|}{$\begin{array}{c}\text { Year } 2000 \\
\text { Percent of Total Agrciultural Area }(\%)\end{array}$}} & \multirow{2}{*}{\multicolumn{4}{|c|}{$\begin{array}{c}\text { Year } 2010 \\
\text { Percent of Total Agrciultural Area ( } \%)\end{array}$}} & \multirow{2}{*}{\multicolumn{4}{|c|}{$\begin{array}{c}\text { Change (2000 to 2010) } \\
\text { Percent of Total Agrciultural Area (\%) }\end{array}$}} \\
\hline & & & & & & & & & & & & & & & & \\
\hline & & 2000 & 2010 & Change & $<10 \%$ & $10-20 \%$ & $20-30 \%$ & $>30 \%$ & $<10 \%$ & $10-20 \%$ & $20-30 \%$ & $>30 \%$ & $<10 \%$ & $10-20 \%$ & $20-30 \%$ & $>30 \%$ \\
\hline Malampa & 1,064 & 66.85 & 70.18 & 3.33 & 0.00 & 0.00 & 0.19 & 99.81 & 0.00 & 0.00 & 0.28 & 99.72 & 0.00 & 0.00 & 0.09 & -0.09 \\
\hline Penama & 310 & 59.89 & 63.22 & 3.33 & 0.00 & 1.29 & 1.29 & 97.42 & 0.00 & 0.00 & 0.00 & 100.00 & 0.00 & -1.29 & -1.29 & 2.58 \\
\hline Sanma & 2,423 & 63.02 & 69.84 & 6.82 & 0.00 & 0.17 & 0.70 & 99.13 & 0.00 & 0.00 & 0.04 & 99.96 & 0.00 & -0.17 & -0.66 & 0.83 \\
\hline Shefa & 107 & 69.65 & 72.25 & 2.60 & 0.00 & 0.00 & 0.00 & 100.00 & 0.00 & 0.00 & 0.00 & 100.00 & 0.00 & 0.00 & 0.00 & 0.00 \\
\hline Torba & 80 & 63.76 & 64.93 & 1.16 & 0.00 & 0.00 & 0.00 & 100.00 & 0.00 & 0.00 & 1.25 & 98.75 & 0.00 & 0.00 & 1.25 & -1.25 \\
\hline Vanuatu - All & 3,984 & 63.99 & 69.38 & 5.39 & 0.00 & 0.20 & 0.58 & 99.22 & 0.00 & 0.00 & 0.13 & 99.88 & 0.00 & -0.20 & -0.45 & 0.65 \\
\hline
\end{tabular}


Asia Pacific Region : Tree Cover on Agricultural Land (2010)

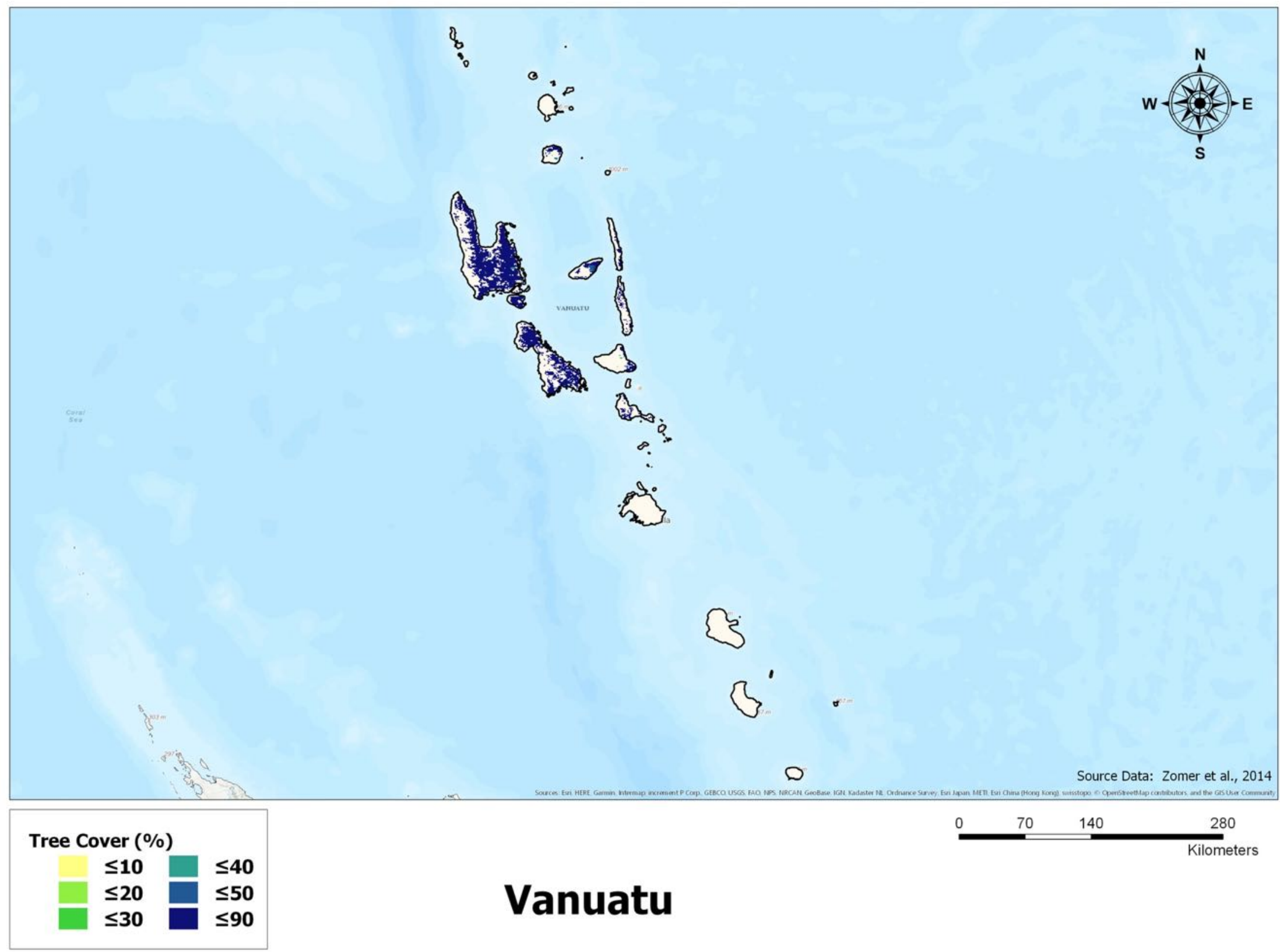


Asia Pacific Region : Change in Tree Cover on Agricultural Land (2000 - 2010)

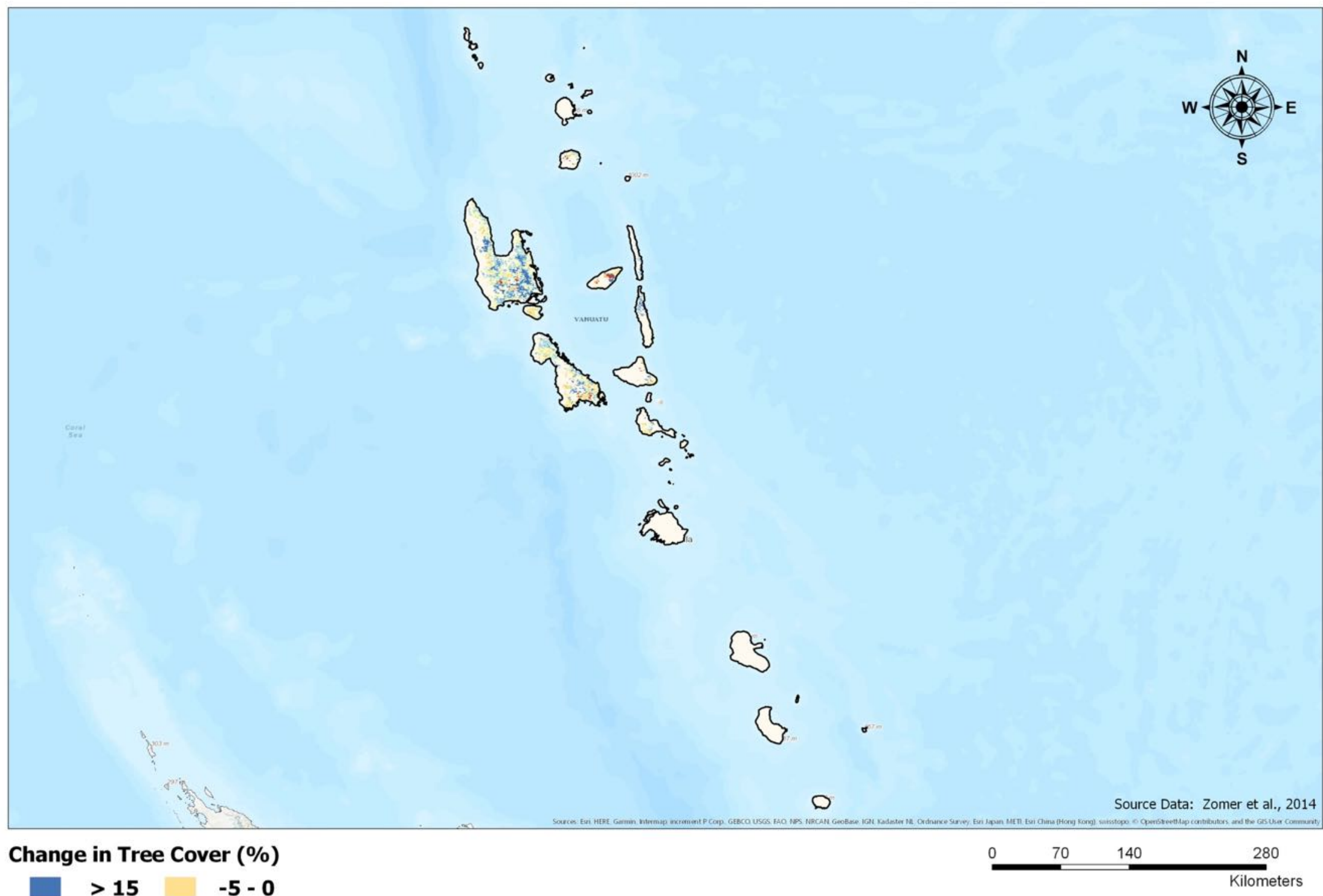

$10-15 \quad-10--5$

$5-10-15--10$

Vanuatu

$0-5 \square \leq-15$ 


\section{Appendix 26: Vietnam}

Average Percent Tree Cover by Province, and Areal Extent of Tree Cover Classes $\left(\mathrm{km}^{2}\right)$

\begin{tabular}{|c|c|c|c|c|c|c|c|c|c|c|c|c|c|c|c|c|c|}
\hline \multirow{4}{*}{ Country } & \multicolumn{2}{|c|}{ Vietnam } & \multirow{2}{*}{\multicolumn{3}{|c|}{\begin{tabular}{|l|} 
Average Tree Cover by Province \\
Mean Tree Cover Percent by Province
\end{tabular}}} & \multicolumn{12}{|c|}{ Areal Extent of Tree Cover Class $\left(\mathrm{km}^{2}\right)$} \\
\hline & \multirow{3}{*}{ Province } & \multirow{3}{*}{ Area_km } & & & & \multirow{2}{*}{\multicolumn{4}{|c|}{ Area by Tree Cover Class $\left(\mathrm{km}^{2}\right)$}} & \multirow{2}{*}{\multicolumn{4}{|c|}{ Area by Tree Cover Class $\left(\mathrm{km}^{2}\right)$}} & & Change $(2$ & 000 to 2010) & \\
\hline & & & \multirow{2}{*}{\multicolumn{3}{|c|}{$\frac{(\%)}{2010}$}} & & & & & & & & & \multicolumn{4}{|c|}{ Area by Tree Cover Class $\left(\mathrm{km}^{2}\right)$} \\
\hline & & & & & & $<10 \%$ & $10-20 \%$ & $20-30 \%$ & $>\mathbf{3 0} \%$ & $<10 \%$ & $10-20 \%$ & $20-30 \%$ & $>30 \%$ & $<10 \%$ & $10-20 \%$ & $20-30 \%$ & $>30 \%$ \\
\hline Vietnam & An Giang & 3,263 & 17.69 & 19.04 & 1.35 & 476 & 1,789 & 862 & 136 & 301 & 1,716 & 1,052 & 194 & -175 & -73 & 190 & 58 \\
\hline Vietnam & Bac Liêu & 2.389 & 11.71 & 11.18 & -0.53 & 1,063 & 1,178 & 127 & 21 & 1.336 & 890 & 155 & 8 & 273 & -288 & 28 & -13 \\
\hline Vietnam & Bắc Giang & 2,930 & 19.17 & 21.41 & 2.24 & 1,589 & 483 & 257 & 601 & 1,134 & 763 & 342 & 691 & -455 & 280 & 85 & 90 \\
\hline Vietnam & Bắc Kan & 3214 & 52.65 & 54.75 & 2.11 & 0 & 23 & 150 & 3.041 & 1 & 28 & 150 & 3.035 & 1 & 5 & 0 & -6 \\
\hline Vietnam & Bắc Ninh & 752 & 7.67 & 660 & -107 & 685 & 67 & 0 & 0 & 726 & 26 & 0 & 0 & 41 & -41 & 0 & 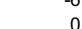 \\
\hline Vietnam & Bến Tre & 1,961 & 14.37 & 21.32 & 6.95 & 608 & 1.031 & 273 & 49 & 541 & 489 & 459 & 472 & -67 & -542 & 186 & 423 \\
\hline Vietnam & Bà Ria - Vũng Tàu & 1253 & 20.36 & 1805 & -2.30 & 345 & 438 & 201 & 269 & 322 & 537 & 225 & 169 & -23 & 99 & $24>$ & -100 \\
\hline Vietnam & Binh Einh & 2232 & 18.53 & 2123 & 270 & 780 & 667 & 365 & 420 & 586 & 772 & 333 & 541 & -194 & 105 & -32 & 121 \\
\hline Vietnam & Bình Dương & 2,359 & 23.40 & 28.69 & 5.29 & 446 & 904 & 381 & 628 & 327 & 442 & 523 & 1,067 & -119 & -462 & 142 & 439 \\
\hline Vietnam & Bình Phước & 3,937 & 27.08 & 28.11 & 1.03 & 281 & 1,147 & 1,112 & 1,397 & 137 & 743 & 1,609 & 1,448 & -144 & -404 & 497 & 51 \\
\hline Vietnam & Bình Thuân & 3,808 & 12.63 & 13.68 & 1.05 & 2,335 & 712 & 274 & 487 & 2.157 & 839 & 282 & 530 & -178 & 127 & 8 & 43 \\
\hline Vietnam & Cần Thơ & 1.290 & 16.95 & 16.88 & -0.07 & 104 & 874 & 297 & 15 & 77 & 956 & 242 & 15 & -27 & 82 & -55 & 0 \\
\hline Vietnam & Cà Mau & 4,179 & 15.61 & 14.79 & -0.82 & 1,219 & 1,994 & 764 & 202 & 1,601 & 1,883 & 359 & 336 & 382 & -111 & -405 & 134 \\
\hline Vietnam & Cao Bằng & 3,272 & 42.65 & 44.71 & 2.06 & 9 & 138 & 525 & 2,600 & 11 & 124 & 523 & 2,614 & 2 & -14 & -2 & 14 \\
\hline Vietnam & Đắk Lắk & 2,349 & 22.75 & 20.89 & -1.86 & 403 & 803 & 553 & 590 & 542 & 837 & 478 & 492 & 139 & 34 & -75 & $-98 \quad \longrightarrow$ \\
\hline Vietnam & Đắk Nông & 885 & 30.23 & 28.23 & -2.00 & 51 & 225 & 224 & 385 & 20 & 232 & 338 & 295 & -31 & 7 & 114 & -90 \\
\hline Vietnam & Đồng Nai & 2,686 & 24.38 & 24.16 & -0.22 & 430 & 940 & 537 & 779 & 346 & 901 & 690 & 749 & -84 & -39 & 153 & -30 \\
\hline Vietnam & Đồng Tháp & 3,339 & 16.88 & 17.85 & 0.97 & 496 & 2.004 & 786 & 53 & 214 & 2,230 & 805 & 90 & -282 & 226 & 19 & 37 \\
\hline Vietnam & Đà Nẵng & 204 & 20.88 & 20.15 & -0.74 & 34 & 77 & 53 & 40 & 61 & 64 & 26 & 53 & 27 & -13 & -27 & 13 \\
\hline Vietnam & Eiện Biên & 2,113 & 40.78 & 36.17 & -4.61 & 49 & 57 & 360 & 1,647 & 15 & 285 & 559 & 1,254 & -34 & 228 & 199 & -393 \\
\hline Vietnam & Gia Lai & 4.766 & 22.04 & 23.53 & 1.49 & 1,292 & 1,366 & 790 & 1318 & 895 & 1.491 & 1.001 & 1379 & -397 & 125 & 211 & $61>$ \\
\hline Vietnam & Hải Dương & 1598 & 10.45 & 8.94 & -1.51 & 1,026 & 519 & 34 & 19 & 1,302 & 224 & 45 & 27 & 276 & -295 & 11 & 8 \\
\hline Vietnam & Hải Phòng & 777 & 11.02 & 8.36 & -2.66 & 397 & 371 & 9 & 0 & 629 & 147 & 1 & 0 & 232 & -224 & -8 & 0 \\
\hline Vietnam & Hâu Giang & 1,564 & 21.19 & 21.99 & 0.80 & 5 & 762 & 727 & 70 & 6 & 688 & 747 & 123 & 1 & -74 & 20 & 53 \\
\hline Vietnam & Hồ Chi Minh & 689 & 14.85 & 14.31 & -0.54 & 257 & 335 & 63 & 34 & 264 & 332 & 55 & 38 & 7 & -3 & -8 & 4 \\
\hline Vietnam & Hà Giang & 4.570 & 38.83 & 38.58 & -0.26 & 93 & 608 & 804 & 3.065 & 118 & 515 & 926 & 3,011 & 25 & -93 & 122 & -54 \\
\hline Vietnam & Hà Nội & 2,638 & 10.85 & 11.19 & 0.34 & 1,906 & 535 & 82 & 115 & 1,960 & 400 & 132 & 146 & 54 & -135 & 50 & 31 \\
\hline Vietnam & Hà Nam & 831 & 10.70 & 10.85 & 0.15 & 613 & 153 & 26 & 39 & 627 & 137 & 25 & 42 & 14 & -16 & -1 & 3 \\
\hline Vietnam & Hà Tỉnh & 2.023 & 13.43 & 17.12 & 3.68 & 1,381 & 320 & 128 & 194 & 762 & 815 & 150 & 296 & -619 & 495 & 22 & 102 \\
\hline Vietnam & Hoà Bình & 2,396 & 36.43 & 36.16 & -0.27 & 75 & 452 & 432 & 1,437 & 36 & 338 & 513 & 1,509 & -39 & -114 & 81 & 72 \\
\hline Vietnam & Hưng Yên & 926 & 7.61 & 6.65 & -0.96 & 842 & 84 & 0 & 0 & 876 & 50 & 0 & 0 & 34 & -34 & 0 & 0 \\
\hline Vietnam & Khánh Hòa & 1,635 & 24.63 & 22.63 & -2.00 & 274 & 508 & 354 & 499 & 261 & 644 & 356 & 374 & -13 & 136 & 2 & -125 \\
\hline Vietnam & Kiên Giang & 4.461 & 19.52 & 16.64 & -2.88 & 113 & 2,744 & 1,447 & 157 & 728 & 2.695 & 895 & 143 & 615 & -49 & -552 & -14 \\
\hline Vietnam & Kon Tum & 1,666 & 29.18 & 29.33 & 0.14 & 188 & 346 & 382 & 750 & 184 & 344 & 410 & 728 & -4 & -2 & 28 & -22 \\
\hline Vietnam & Lang Sơn & 3.732 & 41.71 & 43.13 & 1.42 & 146 & 307 & 428 & 2.851 & 123 & 255 & 386 & 2,968 & -23 & -52 & -42 & 117 \\
\hline Vietnam & Lai Châu & 1,367 & 39.53 & 35.29 & -4.23 & 4 & 56 & 313 & 994 & 12 & 130 & 445 & 780 & 8 & 74 & 132 & -214 \\
\hline Vietnam & Lâm Đồng & 1,368 & 23.86 & 27.18 & 3.32 & 191 & 482 & 338 & 357 & 108 & 325 & 435 & 500 & -83 & -157 & 97 & 143 \\
\hline Vietnam & Lào Cai & 3,357 & 36.12 & 34.97 & -1.14 & 23 & 392 & 901 & 2,041 & 31 & 363 & 1,079 & 1.884 & 8 & -29 & 178 & -157 \\
\hline Vietnam & Long An & 3,996 & 14.73 & 15.64 & 0.91 & 891 & 2,592 & 457 & 56 & 817 & 2,482 & 585 & 112 & -74 & -110 & 128 & 56 \\
\hline Vietnam & Nam Einh & 1,358 & 8.11 & 9.12 & 1.02 & 1,229 & 129 & 0 & 0 & 1,010 & 348 & 0 & 0 & -219 & 219 & 0 & 0 \\
\hline Vietnam & Nghệ An & 4,668 & 30.44 & 29.53 & -0.91 & 1,739 & 697 & 205 & 2,027 & 1,451 & 909 & 311 & 1,997 & -288 & 212 & 106 & -30 \\
\hline Vietnam & Ninh Binh & 1,002 & 12.89 & 12.52 & -0.37 & 636 & 239 & 46 & 81 & 678 & 196 & 45 & 83 & 42 & -43 & -1 & 2 \\
\hline Vietnam & Ninh Thuận & 1,701 & 19.44 & 19.12 & -0.32 & 782 & 235 & 172 & 512 & 775 & 278 & 165 & 483 & -7 & 43 & -7 & -29 \\
\hline Vietnam & Phú Thọ & 2,980 & 25.08 & 26.89 & 1.81 & 472 & 1,028 & 628 & 852 & 414 & 847 & 681 & 1,038 & -58 & -181 & 53 & 186 \\
\hline Vietnam & Phú Yên & 1,975 & 21.48 & 20.44 & -1.04 & 356 & 761 & 454 & 404 & 427 & 825 & 341 & 382 & 71 & 64 & -113 & -22 \\
\hline Vietnam & Quảng Bình & 1,756 & 22.22 & 24.99 & 2.76 & 710 & 359 & 194 & 493 & 643 & 341 & 190 & 582 & -67 & -18 & -4 & 89 \\
\hline Vietnam & Quảng Nam & 2,053 & 17.74 & 17.71 & -0.04 & 543 & 878 & 362 & 270 & 643 & 787 & 294 & 329 & 100 & -91 & -68 & 59 \\
\hline
\end{tabular}




\begin{tabular}{|c|c|c|c|c|c|c|c|c|c|c|c|c|c|c|c|c|}
\hline \multicolumn{2}{|c|}{ Vietnam } & \multirow{2}{*}{\multicolumn{3}{|c|}{\begin{tabular}{|c|} 
Average Tree Cover by Province \\
Mean Tree Cover Percent by Province
\end{tabular}}} & \multicolumn{12}{|c|}{ Areal Extent of Tree Cover Class $\left(\mathrm{km}^{2}\right)$} \\
\hline \multirow{3}{*}{ Province } & \multirow{3}{*}{ Area_km } & & & & \multirow{2}{*}{\multicolumn{4}{|c|}{$\begin{array}{c}\text { Year } 2000 \\
\end{array}$}} & \multirow{2}{*}{\multicolumn{4}{|c|}{$\frac{\text { Year } 2010}{\text { Area by Tree Cover Class }\left(\mathrm{km}^{2}\right)}$}} & \multirow{2}{*}{\multicolumn{4}{|c|}{ Area by Tree Cover Class $\left(\mathrm{km}^{2}\right)$}} \\
\hline & & \multicolumn{3}{|c|}{$\begin{array}{c}\text { Mean Tree Cover Percent by Province } \\
(\%)\end{array}$} & & & & & & & & & & & & \\
\hline & & 2000 & 2010 & Change & $<10 \%$ & $10-20 \%$ & $20-30 \%$ & $>30 \%$ & $<10 \%$ & $10-20 \%$ & $20-30 \%$ & $>30 \%$ & $<10 \%$ & $10-20 \%$ & $20-30 \%$ & $>30 \%$ \\
\hline Quảng Ngãi & 1,623 & 19.39 & 18.80 & -0.60 & 328 & 746 & 281 & 268 & 389 & 712 & 237 & 285 & 61 & -34 & -44 & 17 \\
\hline Quảng Ninh & 2,449 & 38.78 & 35.63 & -3.15 & 212 & 392 & 254 & 1,591 & 332 & 361 & 289 & 1,467 & 120 & -31 & 35 & -124 \\
\hline Quảng Trị & 1,469 & 23.36 & 24.15 & 0.79 & 434 & 392 & 199 & 444 & 344 & 444 & 243 & 438 & -90 & 52 & 44 & -6 \\
\hline Sóc Trăng & 3,068 & 14.56 & 16.08 & 1.52 & 853 & 1,746 & 416 & 53 & 956 & 1,373 & 523 & 216 & 103 & -373 & 107 & 163 \\
\hline Sơn La & 4,340 & 40.52 & 34.21 & -6.31 & 29 & 313 & 737 & 3,261 & 68 & 784 & 1,256 & 2,232 & 39 & 471 & 519 & $-1,029$ \\
\hline Tây Ninh & 3,060 & 15.39 & 18.05 & 2.66 & 1,463 & 933 & 308 & 356 & 987 & 1,232 & 361 & 480 & -476 & 299 & 53 & 124 \\
\hline Thừa Thiên Huế & 677 & 15.92 & 18.10 & 2.18 & 288 & 226 & 84 & 79 & 191 & 324 & 61 & 101 & -97 & 98 & -23 & 22 \\
\hline Thái Bình & 1,414 & 9.27 & 7.62 & -1.65 & 1,016 & 397 & 1 & 0 & 1,283 & 130 & 1 & 0 & 267 & -267 & 0 & 0 \\
\hline Thái Nguyên & 2,426 & 31.50 & 34.15 & 2.66 & 360 & 436 & 496 & 1,134 & 210 & 519 & 426 & 1,271 & -150 & 83 & -70 & 137 \\
\hline Thanh Hóa & 4,586 & 25.48 & 25.46 & -0.02 & 1,806 & 955 & 226 & 1,599 & 1,626 & 1,076 & 364 & 1,520 & -180 & 121 & 138 & -79 \\
\hline Tiền Giang & 2,138 & 17.31 & 21.49 & 4.18 & 178 & 1,416 & 516 & 28 & 82 & 986 & 830 & 240 & -96 & -430 & 314 & 212 \\
\hline Trà Vinh & 2,102 & 14.34 & 15.22 & 0.89 & 529 & 1,318 & 253 & 2 & 714 & 879 & 445 & 64 & 185 & -439 & 192 & 62 \\
\hline Tuyên Quang & 5,067 & 42.93 & 43.51 & 0.58 & 52 & 515 & 904 & 3,596 & 42 & 422 & 778 & 3,825 & -10 & -93 & -126 & 229 \\
\hline Vĩnh Long & 1,397 & 17.41 & 19.87 & 2.46 & 78 & 962 & 356 & 1 & 26 & 860 & 425 & 86 & -52 & -102 & 69 & 85 \\
\hline Vĩnh Phúc & 1,118 & 14.26 & 14.57 & 0.31 & 551 & 400 & 64 & 103 & 724 & 198 & 68 & 128 & 173 & -202 & 4 & 25 \\
\hline Yên Bái & 3,729 & 40.16 & 39.04 & -1.12 & 5 & 390 & 835 & 2,499 & 20 & 386 & 810 & 2,513 & 15 & -4 & -25 & 14 \\
\hline Vietnam - All & 150,934 & 24.92 & 25.19 & 0.27 & 35,770 & 44,347 & 23,807 & 47,010 & 34,230 & 42,350 & 26,480 & 47,874 & $-1,540$ & $-1,997$ & 2,673 & 864 \\
\hline
\end{tabular}




\begin{tabular}{|c|c|c|c|c|c|c|c|c|c|c|c|c|c|c|c|c|}
\hline \multicolumn{2}{|c|}{ Vietnam } & \multirow{3}{*}{\multicolumn{3}{|c|}{$\begin{array}{c}\text { Average Tree Cover by Province } \\
\text { Percent Tree Cover }\end{array}$}} & \multicolumn{12}{|c|}{ Areal Extent of Tree Cover Class as a Percent of Total Agricultural Area (\%) } \\
\hline \multirow{3}{*}{ Province } & \multirow{3}{*}{ Area_km } & & & & \multirow{2}{*}{\multicolumn{4}{|c|}{$\begin{array}{c}\text { Year } 2000 \\
\text { Percent of Total Agrciultural Area }(\%) \\
\end{array}$}} & \multirow{2}{*}{\multicolumn{4}{|c|}{$\begin{array}{c}\text { Year } 2010 \\
\text { Percent of Total Agrciultural Area (\%) }\end{array}$}} & \multirow{2}{*}{\multicolumn{4}{|c|}{$\begin{array}{c}\text { Change (2000 to 2010) } \\
\text { Percent of Total Agrciultural Area (\%) }\end{array}$}} \\
\hline & & & & & & & & & & & & & & & & \\
\hline & & 2000 & 2010 & Change & $<10 \%$ & $10-20 \%$ & $20-30 \%$ & $>30 \%$ & $<10 \%$ & $10-20 \%$ & $20-30 \%$ & $>30 \%$ & $<10 \%$ & $10-20 \%$ & $20-30 \%$ & $>30 \%$ \\
\hline An Giang & 3,263 & 17.69 & 19.04 & 1.35 & 14.59 & 54.83 & 26.42 & 4.17 & 9.22 & 52.59 & 32.24 & 5.95 & -5.36 & -2.24 & 5.82 & 1.78 \\
\hline Bạc Liêu & 2,389 & 11.71 & 11.18 & -0.53 & 44.50 & 49.31 & 5.32 & 0.88 & 55.92 & 37.25 & 6.49 & 0.33 & 11.43 & -12.06 & 1.17 & -0.54 \\
\hline Bắc Giang & 2,930 & 19.17 & 21.41 & 2.24 & 54.23 & 16.48 & 8.77 & 20.51 & 38.70 & 26.04 & 11.67 & 23.58 & -15.53 & 9.56 & 2.90 & 3.07 \\
\hline Bắc Kạn & 3,214 & 52.65 & 54.75 & 2.11 & 0.00 & 0.72 & 4.67 & 94.62 & 0.03 & 0.87 & 4.67 & 94.43 & 0.03 & 0.16 & 0.00 & -0.19 \\
\hline Bắc Ninh & 752 & 7.67 & 6.60 & -1.07 & 91.09 & 8.91 & 0.00 & 0.00 & 96.54 & 3.46 & 0.00 & 0.00 & 5.45 & -5.45 & 0.00 & 0.00 \\
\hline Bến Tre & 1,961 & 14.37 & 21.32 & 6.95 & 31.00 & 52.58 & 13.92 & 2.50 & 27.59 & 24.94 & 23.41 & 24.07 & -3.42 & -27.64 & 9.49 & 21.57 \\
\hline Bà Rịa - Vũng Tàu & 1,253 & 20.36 & 18.05 & -2.30 & 27.53 & 34.96 & 16.04 & 21.47 & 25.70 & 42.86 & 17.96 & 13.49 & -1.84 & 7.90 & 1.92 & -7.98 \\
\hline Bình Định & 2,232 & 18.53 & 21.23 & 2.70 & 34.95 & 29.88 & 16.35 & 18.82 & 26.25 & 34.59 & 14.92 & 24.24 & -8.69 & 4.70 & -1.43 & 5.42 \\
\hline Bình Dương & 2,359 & 23.40 & 28.69 & 5.29 & 18.91 & 38.32 & 16.15 & 26.62 & 13.86 & 18.74 & 22.17 & 45.23 & -5.04 & -19.58 & 6.02 & 18.61 \\
\hline Bình Phước & 3,937 & 27.08 & 28.11 & 1.03 & 7.14 & 29.13 & 28.25 & 35.48 & 3.48 & 18.87 & 40.87 & 36.78 & -3.66 & -10.26 & 12.62 & 1.30 \\
\hline Bình Thuận & 3,808 & 12.63 & 13.68 & 1.05 & 61.32 & 18.70 & 7.20 & 12.79 & 56.64 & 22.03 & 7.41 & 13.92 & -4.67 & 3.34 & 0.21 & 1.13 \\
\hline Cần Tho & 1,290 & 16.95 & 16.88 & -0.07 & 8.06 & 67.75 & 23.02 & 1.16 & 5.97 & 74.11 & 18.76 & 1.16 & -2.09 & 6.36 & -4.26 & 0.00 \\
\hline Cà Mau & 4,179 & 15.61 & 14.79 & -0.82 & 29.17 & 47.72 & 18.28 & 4.83 & 38.31 & 45.06 & 8.59 & 8.04 & 9.14 & -2.66 & -9.69 & 3.21 \\
\hline Cao Bằng & 3,272 & 42.65 & 44.71 & 2.06 & 0.28 & 4.22 & 16.05 & 79.46 & 0.34 & 3.79 & 15.98 & 79.89 & 0.06 & -0.43 & -0.06 & 0.43 \\
\hline Đắk Lắk & 2,349 & 22.75 & 20.89 & -1.86 & 17.16 & 34.19 & 23.54 & 25.12 & 23.07 & 35.63 & 20.35 & 20.95 & 5.92 & 1.45 & -3.19 & -4.17 \\
\hline Đắk Nông & 885 & 30.23 & 28.23 & -2.00 & 5.76 & 25.42 & 25.31 & 43.50 & 2.26 & 26.21 & 38.19 & 33.33 & -3.50 & 0.79 & 12.88 & -10.17 \\
\hline Đồng Nai & 2,686 & 24.38 & 24.16 & -0.22 & 16.01 & 35.00 & 19.99 & 29.00 & 12.88 & 33.54 & 25.69 & 27.89 & -3.13 & -1.45 & 5.70 & -1.12 \\
\hline Đồng Tháp & 3,339 & 16.88 & 17.85 & 0.97 & 14.85 & 60.02 & 23.54 & 1.59 & 6.41 & 66.79 & 24.11 & 2.70 & -8.45 & 6.77 & 0.57 & 1.11 \\
\hline Đà Nẵng & 204 & 20.88 & 20.15 & -0.74 & 16.67 & 37.75 & 25.98 & 19.61 & 29.90 & 31.37 & 12.75 & 25.98 & 13.24 & -6.37 & -13.24 & 6.37 \\
\hline Điện Biên & 2,113 & 40.78 & 36.17 & -4.61 & 2.32 & 2.70 & 17.04 & 77.95 & 0.71 & 13.49 & 26.46 & 59.35 & -1.61 & 10.79 & 9.42 & -18.60 \\
\hline Gia Lai & 4,766 & 22.04 & 23.53 & 1.49 & 27.11 & 28.66 & 16.58 & 27.65 & 18.78 & 31.28 & 21.00 & 28.93 & -8.33 & 2.62 & 4.43 & 1.28 \\
\hline Hải Dương & 1,598 & 10.45 & 8.94 & -1.51 & 64.21 & 32.48 & 2.13 & 1.19 & 81.48 & 14.02 & 2.82 & 1.69 & 17.27 & -18.46 & 0.69 & 0.50 \\
\hline Hải Phòng & 777 & 11.02 & 8.36 & -2.66 & 51.09 & 47.75 & 1.16 & 0.00 & 80.95 & 18.92 & 0.13 & 0.00 & 29.86 & -28.83 & -1.03 & 0.00 \\
\hline Hậu Giang & 1,564 & 21.19 & 21.99 & 0.80 & 0.32 & 48.72 & 46.48 & 4.48 & 0.38 & 43.99 & 47.76 & 7.86 & 0.06 & -4.73 & 1.28 & 3.39 \\
\hline Hồ Chí Minh & 689 & 14.85 & 14.31 & -0.54 & 37.30 & 48.62 & 9.14 & 4.93 & 38.32 & 48.19 & 7.98 & 5.52 & 1.02 & -0.44 & -1.16 & 0.58 \\
\hline Hà Giang & 4,570 & 38.83 & 38.58 & -0.26 & 2.04 & 13.30 & 17.59 & 67.07 & 2.58 & 11.27 & 20.26 & 65.89 & 0.55 & -2.04 & 2.67 & -1.18 \\
\hline Hà Nội & 2,638 & 10.85 & 11.19 & 0.34 & 72.25 & 20.28 & 3.11 & 4.36 & 74.30 & 15.16 & 5.00 & 5.53 & 2.05 & -5.12 & 1.90 & 1.18 \\
\hline Hà Nam & 831 & 10.70 & 10.85 & 0.15 & 73.77 & 18.41 & 3.13 & 4.69 & 75.45 & 16.49 & 3.01 & 5.05 & 1.68 & -1.93 & -0.12 & 0.36 \\
\hline Hà Tĩnh & 2,023 & 13.43 & 17.12 & 3.68 & 68.27 & 15.82 & 6.33 & 9.59 & 37.67 & 40.29 & 7.41 & 14.63 & -30.60 & 24.47 & 1.09 & 5.04 \\
\hline Hoà Bình & 2,396 & 36.43 & 36.16 & -0.27 & 3.13 & 18.86 & 18.03 & 59.98 & 1.50 & 14.11 & 21.41 & 62.98 & -1.63 & -4.76 & 3.38 & 3.01 \\
\hline Hưng Yên & 926 & 7.61 & 6.65 & -0.96 & 90.93 & 9.07 & 0.00 & 0.00 & 94.60 & 5.40 & 0.00 & 0.00 & 3.67 & -3.67 & 0.00 & 0.00 \\
\hline Khánh Hòa & 1,635 & 24.63 & 22.63 & -2.00 & 16.76 & 31.07 & 21.65 & 30.52 & 15.96 & 39.39 & 21.77 & 22.87 & -0.80 & 8.32 & 0.12 & -7.65 \\
\hline Kiên Giang & 4,461 & 19.52 & 16.64 & -2.88 & 2.53 & 61.51 & 32.44 & 3.52 & 16.32 & 60.41 & 20.06 & 3.21 & 13.79 & -1.10 & -12.37 & -0.31 \\
\hline Kon Tum & 1,666 & 29.18 & 29.33 & 0.14 & 11.28 & 20.77 & 22.93 & 45.02 & 11.04 & 20.65 & 24.61 & 43.70 & -0.24 & -0.12 & 1.68 & -1.32 \\
\hline Lạng Sơn & 3,732 & 41.71 & 43.13 & 1.42 & 3.91 & 8.23 & 11.47 & 76.39 & 3.30 & 6.83 & 10.34 & 79.53 & -0.62 & -1.39 & -1.13 & 3.14 \\
\hline Lai Châu & 1,367 & 39.53 & 35.29 & -4.23 & 0.29 & 4.10 & 22.90 & 72.71 & 0.88 & 9.51 & 32.55 & 57.06 & 0.59 & 5.41 & 9.66 & -15.65 \\
\hline Lâm Đồng & 1,368 & 23.86 & 27.18 & 3.32 & 13.96 & 35.23 & 24.71 & 26.10 & 7.89 & 23.76 & 31.80 & 36.55 & -6.07 & -11.48 & 7.09 & 10.45 \\
\hline Lào Cai & 3,357 & 36.12 & 34.97 & -1.14 & 0.69 & 11.68 & 26.84 & 60.80 & 0.92 & 10.81 & 32.14 & 56.12 & 0.24 & -0.86 & 5.30 & -4.68 \\
\hline Long An & 3,996 & 14.73 & 15.64 & 0.91 & 22.30 & 64.87 & 11.44 & 1.40 & 20.45 & 62.11 & 14.64 & 2.80 & -1.85 & -2.75 & 3.20 & 1.40 \\
\hline Nam Định & 1,358 & 8.11 & 9.12 & 1.02 & 90.50 & 9.50 & 0.00 & 0.00 & 74.37 & 25.63 & 0.00 & 0.00 & -16.13 & 16.13 & 0.00 & 0.00 \\
\hline Nghệ An & 4,668 & 30.44 & 29.53 & -0.91 & 37.25 & 14.93 & 4.39 & 43.42 & 31.08 & 19.47 & 6.66 & 42.78 & -6.17 & 4.54 & 2.27 & -0.64 \\
\hline Ninh Bình & 1,002 & 12.89 & 12.52 & -0.37 & 63.47 & 23.85 & 4.59 & 8.08 & 67.67 & 19.56 & 4.49 & 8.28 & 4.19 & -4.29 & -0.10 & 0.20 \\
\hline Ninh Thuận & 1,701 & 19.44 & 19.12 & -0.32 & 45.97 & 13.82 & 10.11 & 30.10 & 45.56 & 16.34 & 9.70 & 28.40 & -0.41 & 2.53 & -0.41 & -1.70 \\
\hline Phú Thọ & 2,980 & 25.08 & 26.89 & 1.81 & 15.84 & 34.50 & 21.07 & 28.59 & 13.89 & 28.42 & 22.85 & 34.83 & -1.95 & -6.07 & 1.78 & 6.24 \\
\hline Phú Yên & 1,975 & 21.48 & 20.44 & -1.04 & 18.03 & 38.53 & 22.99 & 20.46 & 21.62 & 41.77 & 17.27 & 19.34 & 3.59 & 3.24 & -5.72 & -1.11 \\
\hline Quảng Bình & 1,756 & 22.22 & 24.99 & 2.76 & 40.43 & 20.44 & 11.05 & 28.08 & 36.62 & 19.42 & 10.82 & 33.14 & -3.82 & -1.03 & -0.23 & 5.07 \\
\hline Quảng Nam & 2.053 & 17.74 & 17.71 & -0.04 & 26.45 & 42.77 & 17.63 & 13.15 & 31.32 & 38.33 & 14.32 & 16.03 & 4.87 & -4.43 & -3.31 & 2.87 \\
\hline
\end{tabular}




\begin{tabular}{|c|c|c|c|c|c|c|c|c|c|c|c|c|c|c|c|c|}
\hline \multicolumn{2}{|c|}{ Vietnam } & \multirow{3}{*}{\multicolumn{3}{|c|}{$\begin{array}{c}\text { Average Tree Cover by Province } \\
\text { Percent Tree Cover } \\
(\%)\end{array}$}} & \multicolumn{12}{|c|}{ Extent of Tree Cover Class as a Percent of Total Agricultural Area (\%) } \\
\hline \multirow{3}{*}{ Province } & \multirow{3}{*}{ Area_km } & & & & \multirow{2}{*}{\multicolumn{4}{|c|}{$\begin{array}{c}\text { Year } 2000 \\
\text { Percent of Total Agrciultural Area ( \%) }\end{array}$}} & \multirow{2}{*}{\multicolumn{4}{|c|}{$\begin{array}{c}\text { Year } 2010 \\
\text { Percent of Total Agrciultural Area (\%) } \\
\end{array}$}} & \multirow{2}{*}{\multicolumn{4}{|c|}{$\begin{array}{c}\text { Change (2000 to } 2010 \text { ) } \\
\text { Percent of Total Agrciultural Area (\%) }\end{array}$}} \\
\hline & & & & & & & & & & & & & & & & \\
\hline & & 2000 & 2010 & Change & $<10 \%$ & $10-20 \%$ & $20-30 \%$ & $>30 \%$ & $<10 \%$ & $10-20 \%$ & $20-30 \%$ & $>30 \%$ & $<10 \%$ & $10-20 \%$ & $20-30 \%$ & $>30 \%$ \\
\hline Quảng Ngãi & 1,623 & 19.39 & 18.80 & -0.60 & 20.21 & 45.96 & 17.31 & 16.51 & 23.97 & 43.87 & 14.60 & 17.56 & 3.76 & -2.09 & -2.71 & 1.05 \\
\hline Quảng Ninh & 2,449 & 38.78 & 35.63 & -3.15 & 8.66 & 16.01 & 10.37 & 64.97 & 13.56 & 14.74 & 11.80 & 59.90 & 4.90 & -1.27 & 1.43 & -5.06 \\
\hline Quảng Trị & 1,469 & 23.36 & 24.15 & 0.79 & 29.54 & 26.69 & 13.55 & 30.22 & 23.42 & 30.22 & 16.54 & 29.82 & -6.13 & 3.54 & 3.00 & -0.41 \\
\hline Sóc Trăng & 3,068 & 14.56 & 16.08 & 1.52 & 27.80 & 56.91 & 13.56 & 1.73 & 31.16 & 44.75 & 17.05 & 7.04 & 3.36 & -12.16 & 3.49 & 5.31 \\
\hline Sơn La & 4,340 & 40.52 & 34.21 & -6.31 & 0.67 & 7.21 & 16.98 & 75.14 & 1.57 & 18.06 & 28.94 & 51.43 & 0.90 & 10.85 & 11.96 & -23.71 \\
\hline Tây Ninh & 3,060 & 15.39 & 18.05 & 2.66 & 47.81 & 30.49 & 10.07 & 11.63 & 32.26 & 40.26 & 11.80 & 15.69 & -15.56 & 9.77 & 1.73 & 4.05 \\
\hline Thừa Thiên Huế & 677 & 15.92 & 18.10 & 2.18 & 42.54 & 33.38 & 12.41 & 11.67 & 28.21 & 47.86 & 9.01 & 14.92 & -14.33 & 14.48 & -3.40 & 3.25 \\
\hline Thái Bình & 1,414 & 9.27 & 7.62 & -1.65 & 71.85 & 28.08 & 0.07 & 0.00 & 90.74 & 9.19 & 0.07 & 0.00 & 18.88 & -18.88 & 0.00 & 0.00 \\
\hline Thái Nguyên & 2,426 & 31.50 & 34.15 & 2.66 & 14.84 & 17.97 & 20.45 & 46.74 & 8.66 & 21.39 & 17.56 & 52.39 & -6.18 & 3.42 & -2.89 & 5.65 \\
\hline Thanh Hóa & 4,586 & 25.48 & 25.46 & -0.02 & 39.38 & 20.82 & 4.93 & 34.87 & 35.46 & 23.46 & 7.94 & 33.14 & -3.93 & 2.64 & 3.01 & -1.72 \\
\hline Tiền Giang & 2,138 & 17.31 & 21.49 & 4.18 & 8.33 & 66.23 & 24.13 & 1.31 & 3.84 & 46.12 & 38.82 & 11.23 & -4.49 & -20.11 & 14.69 & 9.92 \\
\hline Trà Vinh & 2,102 & 14.34 & 15.22 & 0.89 & 25.17 & 62.70 & 12.04 & 0.10 & 33.97 & 41.82 & 21.17 & 3.04 & 8.80 & -20.89 & 9.13 & 2.95 \\
\hline Tuyên Quang & 5,067 & 42.93 & 43.51 & 0.58 & 1.03 & 10.16 & 17.84 & 70.97 & 0.83 & 8.33 & 15.35 & 75.49 & -0.20 & -1.84 & -2.49 & 4.52 \\
\hline Vĩnh Long & 1,397 & 17.41 & 19.87 & 2.46 & 5.58 & 68.86 & 25.48 & 0.07 & 1.86 & 61.56 & 30.42 & 6.16 & -3.72 & -7.30 & 4.94 & 6.08 \\
\hline Vĩnh Phúc & 1,118 & 14.26 & 14.57 & 0.31 & 49.28 & 35.78 & 5.72 & 9.21 & 64.76 & 17.71 & 6.08 & 11.45 & 15.47 & -18.07 & 0.36 & 2.24 \\
\hline Yên Bái & 3,729 & 40.16 & 39.04 & -1.12 & 0.13 & 10.46 & 22.39 & 67.02 & 0.54 & 10.35 & 21.72 & 67.39 & 0.40 & -0.11 & -0.67 & 0.38 \\
\hline Vietnam - All & 150,934 & 24.92 & 25.19 & 0.27 & 23.70 & 29.38 & 15.77 & 31.15 & 22.68 & 28.06 & 17.54 & 31.72 & -1.02 & -1.32 & 1.77 & 0.57 \\
\hline
\end{tabular}


Asia Pacific Region : Tree Cover on Agricultural Land (2010)

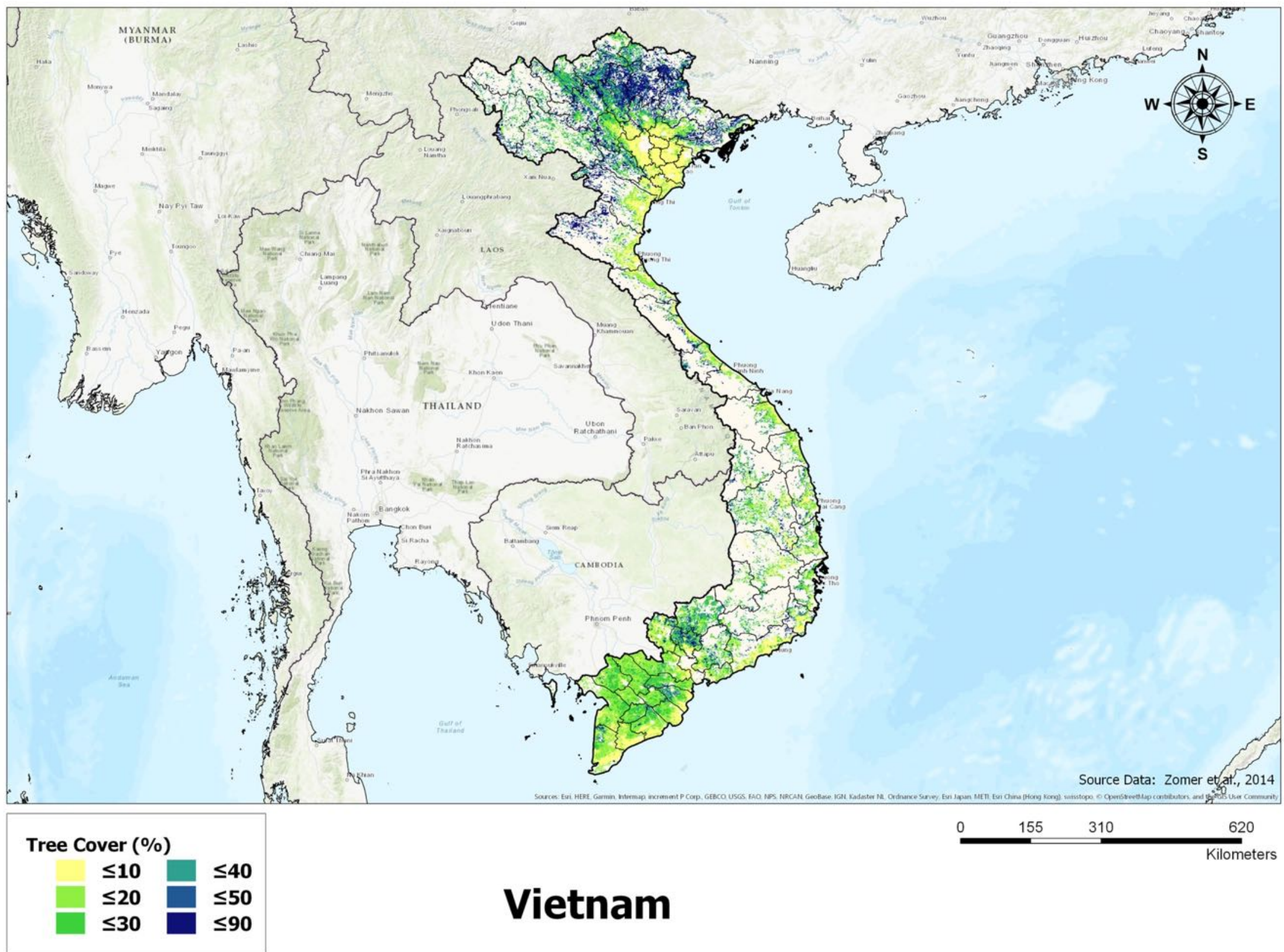


Asia Pacific Region : Change in Tree Cover on Agricultural Land (2000 - 2010)

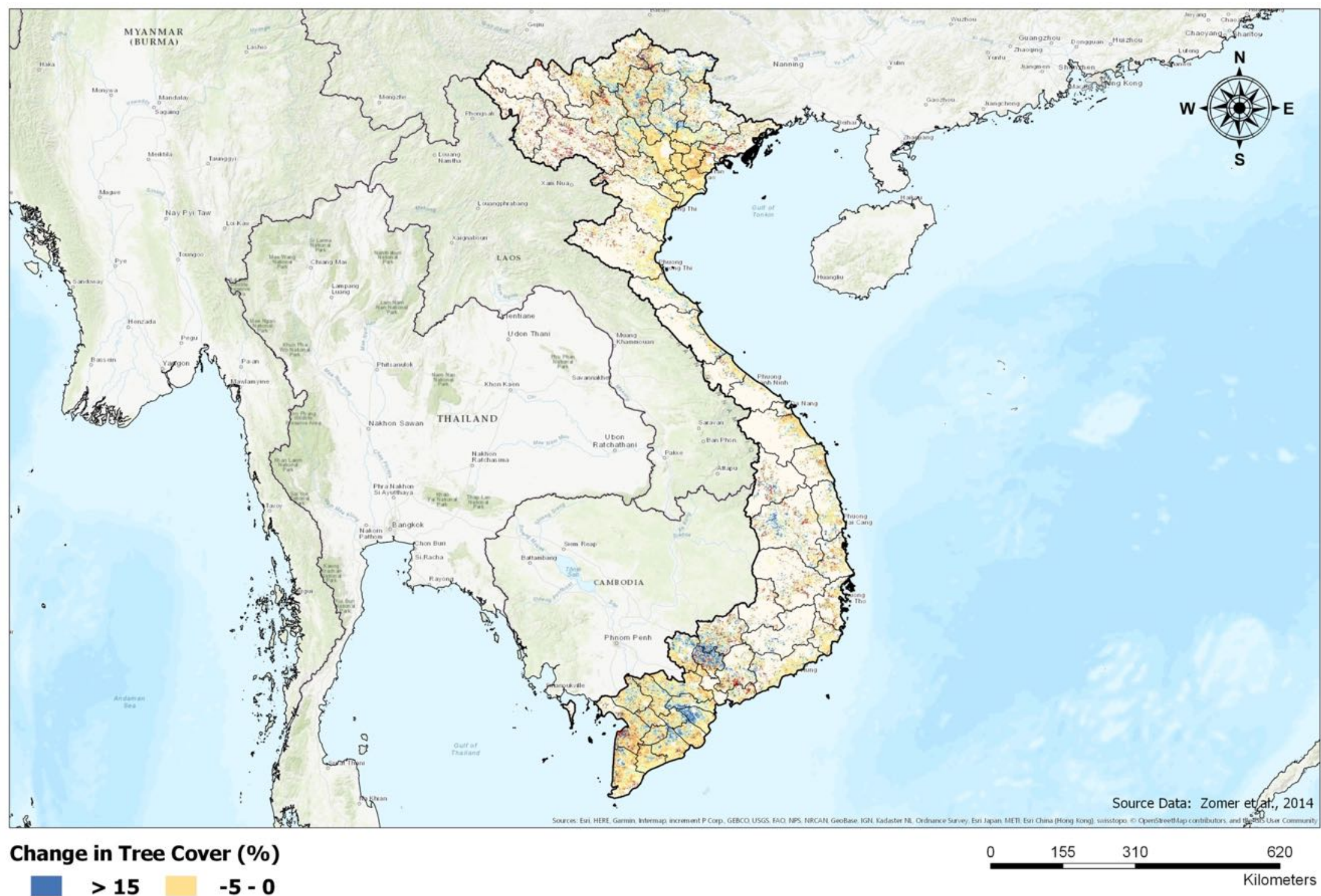
$>15 \quad-5-0$
$10-15 \quad-10--5$
$5-10-15--10$
$0-5 \square \leq-15$

Vietnam 


\section{WORKING PAPERS WITH DOIs}

2005

1. Agroforestry in the drylands of eastern Africa: a call to action

2. Biodiversity conservation through agroforestry: managing tree species diversity within a network of community-based, nongovernmental, governmental and research organizations in western Kenya.

3. Invasion of prosopis juliflora and local livelihoods: Case study from the Lake Baringo area of Kenya

4. Leadership for change in farmers organizations: Training report: Ridar Hotel, Kampala, 29th March to 2nd April 2005.

5. Domestication des espèces agroforestières au Sahel : situation actuelle et perspectives

6. Relevé des données de biodiversité ligneuse: Manuel du projet biodiversité des parcs agroforestiers au Sahel

7. Improved land management in the Lake Victoria Basin: TransVic Project's draft report.

8. Livelihood capital, strategies and outcomes in the Taita hills of Kenya

9. Les espèces ligneuses et leurs usages: Les préférences des paysans dans le Cercle de Ségou, au Mali

10. La biodiversité des espèces ligneuses: Diversité arborée et unités de gestion du terroir dans le Cercle de Ségou, au Mali

2006

11. Bird diversity and land use on the slopes of Mt. Kilimanjaro and the adjacent plains, Tanzania

12. Water, women and local social organization in the Western Kenya Highlands

13. Highlights of ongoing research of the World Agroforestry Centre in Indonesia

14. Prospects of adoption of tree-based systems in a rural landscape and its likely impacts on carbon stocks and farmers' welfare: The FALLOW Model Application in Muara Sungkai, Lampung, Sumatra, in a 'Clean Development Mechanism' context

15. Equipping integrated natural resource managers for healthy Agroforestry landscapes.

17. Agro-biodiversity and CGIAR tree and forest science: approaches and examples from Sumatra.

18. Improving land management in eastern and southern Africa: A review of policies.

19. Farm and household economic study of Kecamatan Nanggung, Kabupaten Bogor, Indonesia: A socio-economic base line study of Agroforestry innovations and livelihood enhancement.

20. Lessons from eastern Africa's unsustainable charcoal business.

21. Evolution of RELMA's approaches to land management: Lessons from two decades of research and development in eastern and southern Africa

22. Participatory watershed management: Lessons from RELMA's work with farmers in eastern Africa.

23. Strengthening farmers' organizations: The experience of RELMA and ULAMP.

24. Promoting rainwater harvesting in eastern and southern Africa.

25. The role of livestock in integrated land management.

26. Status of carbon sequestration projects in Africa: Potential benefits and challenges to scaling up. 
27. Social and Environmental Trade-Offs in Tree Species Selection: A Methodology for Identifying Niche Incompatibilities in Agroforestry [Appears as AHI Working Paper no. 9]

28. Managing tradeoffs in agroforestry: From conflict to collaboration in natural resource management. [Appears as AHI Working Paper no. 10]

29. Essai d'analyse de la prise en compte des systemes agroforestiers pa les legislations forestieres au Sahel: Cas du Burkina Faso, du Mali, du Niger et du Senegal.

30. Etat de la recherche agroforestière au Rwanda etude bibliographique, période 1987-2003

2007

31. Science and technological innovations for improving soil fertility and management in Africa: A report for NEPAD's Science and Technology Forum.

32. Compensation and rewards for environmental services.

33. Latin American regional workshop report compensation.

34. Asia regional workshop on compensation ecosystem services.

35. Report of African regional workshop on compensation ecosystem services.

36. Exploring the inter-linkages among and between compensation and rewards for ecosystem services CRES and human well-being

37. Criteria and indicators for environmental service compensation and reward mechanisms: realistic, voluntary, conditional and pro-poor

38. The conditions for effective mechanisms of compensation and rewards for environmental services.

39. Organization and governance for fostering Pro-Poor Compensation for Environmental Services.

40. How important are different types of compensation and reward mechanisms shaping poverty and ecosystem services across Africa, Asia \& Latin America over the Next two decades?

41. Risk mitigation in contract farming: The case of poultry, cotton, woodfuel and cereals in East Africa.

42. The RELMA savings and credit experiences: Sowing the seed of sustainability

43. Yatich J., Policy and institutional context for NRM in Kenya: Challenges and opportunities for Landcare.

44. Nina-Nina Adoung Nasional di So! Field test of rapid land tenure assessment (RATA) in the Batang Toru Watershed, North Sumatera.

45. Is Hutan Tanaman Rakyat a new paradigm in community based tree planting in Indonesia?

46. Socio-Economic aspects of brackish water aquaculture (Tambak) production in Nanggroe Aceh Darrusalam.

47. Farmer livelihoods in the humid forest and moist savannah zones of Cameroon.

48. Domestication, genre et vulnérabilité : Participation des femmes, des Jeunes et des catégories les plus pauvres à la domestication des arbres agroforestiers au Cameroun.

49. Land tenure and management in the districts around Mt Elgon: An assessment presented to the Mt Elgon ecosystem conservation programme.

50. The production and marketing of leaf meal from fodder shrubs in Tanga, Tanzania: A pro-poor enterprise for improving livestock productivity.

51. Buyers Perspective on Environmental Services (ES) and Commoditization as an approach to liberate ES markets in the Philippines. 
52. Towards Towards community-driven conservation in southwest China: Reconciling state and local perceptions.

53. Biofuels in China: An Analysis of the Opportunities and Challenges of Jatropha curcas in Southwest China.

54. Jatropha curcas biodiesel production in Kenya: Economics and potential value chain development for smallholder farmers

55. Livelihoods and Forest Resources in Aceh and Nias for a Sustainable Forest Resource Management and Economic Progress

56. Agroforestry on the interface of Orangutan Conservation and Sustainable Livelihoods in Batang Toru, North Sumatra.

2008

57. Assessing Hydrological Situation of Kapuas Hulu Basin, Kapuas Hulu Regency, West Kalimantan.

58. Assessing the Hydrological Situation of Talau Watershed, Belu Regency, East Nusa Tenggara.

59. Kajian Kondisi Hidrologis DAS Talau, Kabupaten Belu, Nusa Tenggara Timur.

60. Kajian Kondisi Hidrologis DAS Kapuas Hulu, Kabupaten Kapuas Hulu, Kalimantan Barat.

61. Lessons learned from community capacity building activities to support agroforest as sustainable economic alternatives in Batang Toru orang utan habitat conservation program (Martini, Endri et al.)

62. Mainstreaming Climate Change in the Philippines.

63. A Conjoint Analysis of Farmer Preferences for Community Forestry Contracts in the Sumber Jaya Watershed, Indonesia.

64. The highlands: a shared water tower in a changing climate and changing Asia

65. Eco-Certification: Can It Deliver Conservation and Development in the Tropics.

66. Designing ecological and biodiversity sampling strategies. Towards mainstreaming climate change in grassland management.

67. Towards mainstreaming climate change in grassland management policies and practices on the Tibetan Plateau

68. An Assessment of the Potential for Carbon Finance in Rangelands

69 ECA Trade-offs Among Ecosystem Services in the Lake Victoria Basin.

69. The last remnants of mega biodiversity in West Java and Banten: an in-depth exploration of RaTA (Rapid Land Tenure Assessment) in Mount Halimun-Salak National Park Indonesia

70. Le business plan d'une petite entreprise rurale de production et de commercialisation des plants des arbres locaux. Cas de quatre pépinières rurales au Cameroun.

71. Les unités de transformation des produits forestiers non ligneux alimentaires au Cameroun. Diagnostic technique et stratégie de développement Honoré Tabuna et Ingratia Kayitavu.

72. Les exportateurs camerounais de safou (Dacryodes edulis) sur le marché sous régional et international. Profil, fonctionnement et stratégies de développement.

73. Impact of the Southeast Asian Network for Agroforestry Education (SEANAFE) on agroforestry education capacity.

74. Setting landscape conservation targets and promoting them through compatible land use in the Philippines.

75. Review of methods for researching multistrata systems. 
76. Study on economical viability of Jatropha curcas L. plantations in Northern Tanzania assessing farmers' prospects via cost-benefit analysis

77. Cooperation in Agroforestry between Ministry of Forestry of Indonesia and International Center for Research in Agroforestry

78. "China's bioenergy future. an analysis through the Lens if Yunnan Province

79. Land tenure and agricultural productivity in Africa: A comparative analysis of the economics literature and recent policy strategies and reforms

80. Boundary organizations, objects and agents: linking knowledge with action in Agroforestry watersheds

81. Reducing emissions from deforestation and forest degradation (REDD) in Indonesia: options and challenges for fair and efficient payment distribution mechanisms

2009

82. Mainstreaming climate change into agricultural education: challenges and perspectives

83. Challenging conventional mindsets and disconnects in conservation: the emerging role of ecoagriculture in Kenya's landscape mosaics

84. Lesson learned RATA garut dan bengkunat: suatu upaya membedah kebijakan pelepasan kawasan hutan dan redistribusi tanah bekas kawasan hutan

85. The emergence of forest land redistribution in Indonesia

86. Commercial opportunities for fruit in Malawi

87. Status of fruit production processing and marketing in Malawi

88. Fraud in tree science

89. Trees on farm: analysis of global extent and geographical patterns of agroforestry

90. The springs of Nyando: water, social organization and livelihoods in Western Kenya

91. Building capacity toward region-wide curriculum and teaching materials development in agroforestry education in Southeast Asia

92. Overview of biomass energy technology in rural Yunnan (Chinese - English abstract)

93. A pro-growth pathway for reducing net GHG emissions in China

94. Analysis of local livelihoods from past to present in the central Kalimantan Ex-Mega Rice Project area

95. Constraints and options to enhancing production of high quality feeds in dairy production in Kenya, Uganda and Rwanda

2010

96. Agroforestry education in the Philippines: status report from the Southeast Asian Network for Agroforestry Education (SEANAFE)

97. Economic viability of Jatropha curcas L. plantations in Northern Tanzania- assessing farmers' prospects via cost-benefit analysis.

98. Hot spot of emission and confusion: land tenure insecurity, contested policies and competing claims in the central Kalimantan Ex-Mega Rice Project area

99. Agroforestry competences and human resources needs in the Philippines

100. CES/COS/CIS paradigms for compensation and rewards to enhance environmental Services 
101. Case study approach to region-wide curriculum and teaching materials development in agroforestry education in Southeast Asia

102. Stewardship agreement to reduce emissions from deforestation and degradation (REDD): Lubuk Beringin's Hutan Desa as the first village forest in Indonesia

103. Landscape dynamics over time and space from ecological perspective

104. Komoditisasi atau koinvestasi jasa lingkungan: skema imbal jasa lingkungan program peduli sungai di DAS Way Besai, Lampung, Indonesia

105. Improving smallholders' rubber quality in Lubuk Beringin, Bungo district, Jambi province, Indonesia: an initial analysis of the financial and social benefits

106. Rapid Carbon Stock Appraisal (RACSA) in Kalahan, Nueva Vizcaya, Philippines

107. Tree domestication by ICRAF and partners in the Peruvian Amazon: lessons learned and future prospects in the domain of the Amazon Initiative eco-regional program

108. Memorias del Taller Nacional: "Iniciativas para Reducir la Deforestación en la region Andino Amazónica", 09 de Abril del 2010. Proyecto REALU Peru

109. Percepciones sobre la Equidad y Eficiencia en la cadena de valor de REDD en Perú -Reporte de Talleres en Ucayali, San Martín y Loreto, 2009. Proyecto REALU-Perú.

110. Reducción de emisiones de todos los Usos del Suelo. Reporte del Proyecto REALU Perú Fase 1

111. Programa Alternativas a la Tumba-y-Quema (ASB) en el Perú. Informe Resumen y Síntesis de la Fase II. 2da. versión revisada

112. Estudio de las cadenas de abastecimiento de germoplasma forestal en la amazonía Boliviana

113. Biodiesel in the Amazon

114. Estudio de mercado de semillas forestales en la amazonía Colombiana

115. Estudio de las cadenas de abastecimiento de germoplasma forestal en Ecuador http://dx.doi.org10.5716/WP10340.PDF

116. How can systems thinking, social capital and social network analysis help programs achieve impact at scale?

117. Energy policies, forests and local communities in the Ucayali Region, Peruvian Amazon

118. NTFPs as a Source of Livelihood Diversification for Local Communities in the Batang Toru Orangutan Conservation Program

119. Studi Biodiversitas: Apakah agroforestry mampu mengkonservasi keanekaragaman hayati di DAS Konto?

120. Estimasi Karbon Tersimpan di Lahan-lahan Pertanian di DAS Konto, Jawa Timur

121. Implementasi Kaji Cepat Hidrologi (RHA) di Hulu DAS Brantas, Jawa Timur. http://dx.doi.org/10.5716/WP10338.PDF

122. Kaji Cepat Hidrologi di Daerah Aliran Sungai Krueng Peusangan, NAD,Sumatra http://dx.doi.org/10.5716/WP10337.PDF

123. A Study of Rapid Hydrological Appraisal in the Krueng Peusangan Watershed, NAD, Sumatra. http://dx.doi.org/10.5716/WP10339.PDF

2011

124. An Assessment of farm timber value chains in Mt Kenya area, Kenya

125. A Comparative financial analysis of current land use systems and implications for the adoption of improved agroforestry in the East Usambaras, Tanzania

126. Agricultural monitoring and evaluation systems 
127. Challenges and opportunities for collaborative landscape governance in the East Usambara Mountains, Tanzania

128. Transforming Knowledge to Enhance Integrated Natural Resource Management Research, Development and Advocacy in the Highlands of Eastern Africa http://dx.doi.org/10.5716/WP11084.PDF

129. Carbon-forestry projects in the Philippines: potential and challenges The Mt Kitanglad Range forest-carbon development http://dx.doi.org10.5716/WP11054.PDF

130. Carbon forestry projects in the Philippines: potential and challenges. The Arakan Forest Corridor forest-carbon project. http://dx.doi.org10.5716/WP11055.PDF

131. Carbon-forestry projects in the Philippines: potential and challenges. The Laguna Lake Development Authority's forest-carbon development project. http://dx.doi.org/10.5716/WP11056.PDF

132. Carbon-forestry projects in the Philippines: potential and challenges. The Quirino forest-carbon development project in Sierra Madre Biodiversity Corridor http://dx.doi.org10.5716/WP11057.PDF

133. Carbon-forestry projects in the Philippines: potential and challenges. The Ikalahan Ancestral Domain forest-carbon development http://dx.doi.org10.5716/WP11058.PDF

134. The Importance of Local Traditional Institutions in the Management of Natural Resources in the Highlands of Eastern Africa. http://dx.doi.org/10.5716/WP11085.PDF

135. Socio-economic assessment of irrigation pilot projects in Rwanda. http://dx.doi.org/10.5716/WP11086.PDF

136. Performance of three rambutan varieties (Nephelium lappaceum L.) on various nursery media. http://dx.doi.org/10.5716/WP11232.PDF

137. Climate change adaptation and social protection in agroforestry systems: enhancing adaptive capacity and minimizing risk of drought in Zambia and Honduras http://dx.doi.org/10.5716/WP11269.PDF

138. Does value chain development contribute to rural poverty reduction? Evidence of asset building by smallholder coffee producers in Nicaragua http://dx.doi.org/10.5716/WP11271.PDF

139. Potential for biofuel feedstock in Kenya. http://dx.doi.org/10.5716/WP11272.PDF

140. Impact of fertilizer trees on maize production and food security in six districts of Malawi. http://dx.doi.org/10.5716/WP11281.PDF

2012

141. Fortalecimiento de capacidades para la gestión del Santuario Nacional Pampa Hermosa: Construyendo las bases para un manejo adaptativo para el desarrollo local. Memorias del Proyecto. http://dx.doi.org/10.5716/WP12005.PDF

142. Understanding rural institutional strengthening: A cross-level policy and institutional framework for sustainable development in Kenya http://dx.doi.org/10.5716/WP12012.PDF

143. Climate change vulnerability of agroforestry http://dx.doi.org/10.5716/WP16722.PDF

144. Rapid assesment of the inner Niger delta of Mali http://dx.doi.org/10.5716/WP12021.PDF

145. Designing an incentive program to reduce on-farm deforestationin the East Usambara Mountains, Tanzania http://dx.doi.org/10.5716/WP12048.PDF

146. Extent of adoption of conservation agriculture and agroforestry in Africa: the case of Tanzania, Kenya, Ghana, and Zambia http://dx.doi.org/10.5716/WP12049.PDF 
147. Policy incentives for scaling up conservation agriculture with trees in Africa: the case of Tanzania, Kenya, Ghana and Zambia http://dx.doi.org/10.5716/WP12050.PDF

148. Commoditized or co-invested environmental services? Rewards for environmental services scheme: River Care program Way Besai watershed, Lampung, Indonesia. http://dx.doi.org/10.5716/WP12051.PDF

149. Assessment of the headwaters of the Blue Nile in Ethiopia. http://dx.doi.org/10.5716/WP12160.PDF

150. Assessment of the uThukela Watershed, Kwazaulu. http://dx.doi.org/10.5716/WP12161.PDF

151. Assessment of the Oum Zessar Watershed of Tunisia. http://dx.doi.org/10.5716/WP12162.PDF

152. Assessment of the Ruwenzori Mountains in Uganda. http://dx.doi.org/10.5716/WP12163.PDF

153. History of agroforestry research and development in Viet Nam. Analysis of research opportunities and gaps. http://dx.doi.org/10.5716/WP12052.PDF

154. REDD+ in Indonesia: a Historical Perspective. http://dx.doi.org/10.5716/WP12053.PDF

155. Agroforestry and Forestry in Sulawesi series: Livelihood strategies and land use system dynamics in South Sulawesi http://dx.doi.org/10.5716/WP12054.PDF

156. Agroforestry and Forestry in Sulawesi series: Livelihood strategies and land use system dynamics in Southeast Sulawesi. http://dx.doi.org/10.5716/WP12055.PDF

157. Agroforestry and Forestry in Sulawesi series: Profitability and land-use systems in South and Southeast Sulawesi. http://dx.doi.org/10.5716/WP12056.PDF

158. Agroforestry and Forestry in Sulawesi series: Gender, livelihoods and land in South and Southeast Sulawesi http://dx.doi.org/10.5716/WP12057.PDF

159. Agroforestry and Forestry in Sulawesi series: Agroforestry extension needs at the community level in AgFor project sites in South and Southeast Sulawesi, Indonesia. http://dx.doi.org/10.5716/WP12058.PDF

160. Agroforestry and Forestry in Sulawesi series: Rapid market appraisal of agricultural, plantation and forestry commodities in South and Southeast Sulawesi. http://dx.doi.org/10.5716/WP12059.PDF

2013

161. Diagnosis of farming systems in the Agroforestry for Livelihoods of Smallholder farmers in Northwestern Viet Nam project http://dx.doi.org/10.5716/WP13033.PDF

162. Ecosystem vulnerability to climate change: a literature review. http://dx.doi.org/10.5716/WP13034.PDF

163. Local capacity for implementing payments for environmental services schemes: lessons from the RUPES project in northeastern Viet Nam http://dx.doi.org/10.5716/WP13046.PDF

164. Seri Agroforestri dan Kehutanan di Sulawesi: Agroforestry dan Kehutanan di Sulawesi: Strategi mata pencaharian dan dinamika sistem penggunaan lahan di Sulawesi Selatan http://dx.doi.org/10.5716/WP13040.PDF

165. Seri Agroforestri dan Kehutanan di Sulawesi: Mata pencaharian dan dinamika sistem penggunaan lahan di Sulawesi Tenggara http://dx.doi.org/10.5716/WP13041.PDF

166. Seri Agroforestri dan Kehutanan di Sulawesi: Profitabilitas sistem penggunaan lahan di Sulawesi Selatan dan Sulawesi Tenggara http://dx.doi.org/10.5716/WP13042.PDF

167. Seri Agroforestri dan Kehutanan di Sulawesi: Gender, mata pencarian dan lahan di Sulawesi Selatan dan Sulawesi Tenggara http://dx.doi.org/10.5716/WP13043.PDF 
168. Seri Agroforestri dan Kehutanan di Sulawesi: Kebutuhan penyuluhan agroforestri pada tingkat masyarakat di lokasi proyek AgFor di Sulawesi Selatan dan Tenggara, Indonesia. http://dx.doi.org/10.5716/WP13044.PDF

169. Seri Agroforestri dan Kehutanan di Sulawesi: Laporan hasil penilaian cepat untuk komoditas pertanian, perkebunan dan kehutanan di Sulawesi Selatan dan Tenggara http://dx.doi.org/10.5716/WP13045.PDF

170. Agroforestry, food and nutritional security http://dx.doi.org/10.5716/WP13054.PDF

171. Stakeholder Preferences over Rewards for Ecosystem Services: Implications for a REDD+ Benefit Distribution System in Viet Nam http://dx.doi.org/10.5716/WP13057.PDF

172. Payments for ecosystem services schemes: project-level insights on benefits for ecosystems and the rural poor http://dx.doi.org/10.5716/WP13001.PDF

173. Good practices for smallholder teak plantations: keys to success http://dx.doi.org/10.5716/WP13246.PDF

174. Market analysis of selected agroforestry products in the Vision for Change Project intervention Zone, Côte d'Ivoire http://dx.doi.org/10.5716/WP13249.PDF

175. Rattan futures in Katingan: why do smallholders abandon or keep their gardens in Indonesia's 'rattan district'? http://dx.doi.org/10.5716/WP13251.PDF

176. Management along a gradient: the case of Southeast Sulawesi's cacao production landscapes http://dx.doi.org/10.5716/WP13265.PDF

2014

177. Are trees buffering ecosystems and livelihoods in agricultural landscapes of the Lower Mekong Basin? Consequences for climate-change adaptation. http://dx.doi.org/10.5716/WP14047.PDF

178. Agroforestry, livestock, fodder production and climate change adaptation and mitigation in East Africa: issues and options. http://dx.doi.org/10.5716/WP14050.PDF

179. Trees on farms: an update and reanalysis of agroforestry's global extent and socio-ecological characteristics. http://dx.doi.org/10.5716/WP14064.PDF

180. Beyond reforestation: an assessment of Vietnam's REDD+ readiness. http://dx.doi.org/10.5716/WP14097.PDF

181. Farmer-to-farmer extension in Kenya: the perspectives of organizations using the approach. http://dx.doi.org/10.5716/WP14380.PDF

182. Farmer-to-farmer extension in Cameroon: a survey of extension organizations. http://dx.doi.org/10.5716/WP14383.PDF

183. Farmer-to-farmer extension approach in Malawi: a survey of organizations: a survey of organizations http://dx.doi.org/10.5716/WP14391.PDF

184. Seri Agroforestri dan Kehutanan di Sulawesi: Kuantifikasi jasa lingkungan air dan karbon pola agroforestri pada hutan rakyat di wilayah sungai Jeneberang

185. Options for Climate-Smart Agriculture at Kaptumo Site in Kenyahttp://dx.doi.org/10.5716/WP14394.PDF

2015

186. Agroforestry for Landscape Restoration and Livelihood Development in Central Asia http://dx.doi.org/10.5716/WP14143.PDF 
187. "Projected Climate Change and Impact on Bioclimatic Conditions in the Central and SouthCentral Asia Region" http://dx.doi.org/10.5716/WP14144.PDF

188. Land Cover Changes, Forest Loss and Degradation in Kutai Barat, Indonesia. http://dx.doi.org/10.5716/WP14145.PDF

189. The Farmer-to-Farmer Extension Approach in Malawi: A Survey of Lead Farmers. http://dx.doi.org/10.5716/WP14152.PDF

190. Evaluating indicators of land degradation and targeting agroforestry interventions in smallholder farming systems in Ethiopia. http://dx.doi.org/10.5716/WP14252.PDF

191. Land health surveillance for identifying land constraints and targeting land management options in smallholder farming systems in Western Cameroon

192. Land health surveillance in four agroecologies in Malawi

193. Cocoa Land Health Surveillance: an evidence-based approach to sustainable management of cocoa landscapes in the Nawa region, South-West Côte d'Ivoire http://dx.doi.org/10.5716/WP14255.PDF

194. Situational analysis report: Xishuangbanna autonomous Dai Prefecture, Yunnan Province, China. http://dx.doi.org/10.5716/WP14255.PDF

195. Farmer-to-farmer extension: a survey of lead farmers in Cameroon. http://dx.doi.org/10.5716/WP15009.PDF

196. From transition fuel to viable energy source Improving sustainability in the sub-Saharan charcoal sector http://dx.doi.org/10.5716/WP15011.PDF

197. Mobilizing Hybrid Knowledge for More Effective Water Governance in the Asian Highlands http://dx.doi.org/10.5716/WP15012.PDF

198. Water Governance in the Asian Highlands http://dx.doi.org/10.5716/WP15013.PDF

199. Assessing the Effectiveness of the Volunteer Farmer Trainer Approach in Dissemination of Livestock Feed Technologies in Kenya vis-à-vis other Information Sources http://dx.doi.org/10.5716/WP15022.PDF

200. The rooted pedon in a dynamic multifunctional landscape: Soil science at the World Agroforestry Centre http://dx.doi.org/10.5716/WP15023.PDF

201. Characterising agro-ecological zones with local knowledge. Case study: Huong Khe district, $\mathrm{Ha}$ Tinh, Viet Nam http://dx.doi.org/10.5716/WP15050.PDF

202. Looking back to look ahead: Insight into the effectiveness and efficiency of selected advisory approaches in the dissemination of agricultural technologies indicative of Conservation Agriculture with Trees in Machakos County, Kenya. http://dx.doi.org/10.5716/WP15065.PDF

203. Pro-poor Biocarbon Projects in Eastern Africa Economic and Institutional Lessons. http://dx.doi.org/10.5716/WP15022.PDF

204. Projected climate change impacts on climatic suitability and geographical distribution of banana and coffee plantations in Nepal. http://dx.doi.org/10.5716/WP15294.PDF

205. Agroforestry and Forestry in Sulawesi series: Smallholders' coffee production and marketing in Indonesia. A case study of two villages in South Sulawesi Province. http://dx.doi.org/10.5716/WP15690.PDF

206. Mobile phone ownership and use of short message service by farmer trainers: a case study of Olkalou and Kaptumo in Kenya http://dx.doi.org/10.5716/WP15691.PDF

207. Associating multivariate climatic descriptors with cereal yields: a case study of Southern Burkina Faso http://dx.doi.org/10.5716/WP15273.PDF

208. Preferences and adoption of livestock feed practices among farmers in dairy management groups in Kenya http://dx.doi.org/10.5716/WP15675.PDF 
209. Scaling up climate-smart agriculture: lessons learned from South Asia and pathways for success http://dx.doi.org/10.5716/WP15720.PDF

210. Agroforestry and Forestry in Sulawesi series: Local perceptions of forest ecosystem services and collaborative formulation of reward mechanisms in South and Southeast Sulawesi http://dx.doi.org/10.5716/WP15721.PDF

211. Potential and challenges in implementing the co-investment of ecosystem services scheme in Buol District, Indonesia. http://dx.doi.org/10.5716/WP15722.PDF

212. Tree diversity and its utilization by the local community in Buol District, Indonesia http://dx.doi.org/10.5716/WP15723.PDF

213 Vulnerability of smallholder farmers and their preferences on farming practices in Buol District, Indonesia http://dx.doi.org/10.5716/WP15724.PDF

214. Dynamics of Land Use/Cover Change and Carbon Emission in Buol District, Indonesia http://dx.doi.org/10.5716/WP15725.PDF

215. Gender perspective in smallholder farming practices in Lantapan, Phillippines. http://dx.doi.org/10.5716/WP15726.PDF

216. Vulnerability of smallholder farmers in Lantapan, Bukidnon. http://dx.doi.org/10.5716/WP15727.PDF

217. Vulnerability and adaptive capacity of smallholder farmers in Ho Ho Sub-watershed, Ha Tinh Province, Vietnam http://dx.doi.org/10.5716/WP15728.PDF

218. Local Knowledge on the role of trees to enhance livelihoods and ecosystem services in northern central Vietnam http://dx.doi.org/10.5716/WP15729.PDF

219. Land-use/cover change in Ho Ho Sub-watershed, Ha Tinh Province, Vietnam. http://dx.doi.org/10.5716/WP15730.PDF

2016

220. Agroforestry and Forestry in Sulawesi series: Evaluation of the Agroforestry Farmer Field Schools on agroforestry management in South and Southeast Sulawesi, Indonesia. http://dx.doi.org/10.5716/WP16002.PDF

221. Farmer-to-farmer extension of livestock feed technologies in Rwanda: A survey of volunteer farmer trainers and organizations. http://dx.doi.org/10.5716/WP16005.PDF

222. Projected Climate Change Impact on Hydrology, Bioclimatic Conditions, and Terrestrial Ecosystems in the Asian Highlands http://dx.doi.org/10.5716/WP16006.PDF

223. Adoption of Agroforestry and its impact on household food security among farmers in Malawi http://dx.doi.org/10.5716/WP16013.PDF

224. Agroforestry and Forestry in Sulawesi series: Information channels for disseminating innovative agroforestry practices to villages in Southern Sulawesi, Indonesia http://dx.doi.org/10.5716/WP16034.PDF

225. Agroforestry and Forestry in Sulawesi series: Unravelling rural migration networks.Landtenure arrangements among Bugis migrant communities in Southeast Sulawesi. http://dx.doi.org/10.5716/WP16035.PDF

226. Agroforestry and Forestry in Sulawesi series: Women's participation in agroforestry: more benefit or burden? A gendered analysis of Gorontalo Province. http://dx.doi.org/10.5716/WP16036.PDF

227. Kajian Kelayakan dan Pengembangan Desain Teknis Rehabilitasi Pesisir di Sulawesi Tengah. http://dx.doi.org/10.5716/WP16037.PDF

228. Selection of son tra clones in North West Vietnam. http://dx.doi.org/10.5716/WP16038.PDF 
229. Growth and fruit yield of seedlings, cuttings and grafts from selected son tra trees in Northwest Vietnam http://dx.doi.org/10.5716/WP16046.PDF

230. Gender-Focused Analysis of Poverty and Vulnerability in Yunnan, China http://dx.doi.org/10.5716/WP16071.PDF

231. Seri Agroforestri dan Kehutanan di Sulawesi: Kebutuhan Penyuluhan Agroforestri untuk Rehabilitasi Lahan di Sumba Timur, Nusa Tenggara Timur, Indonesia. http://dx.doi.org/10.5716/WP16077.PDF

232. Agroforestry and Forestry in Sulawesi series: Agroforestry extension needs for land rehabilitation in East Sumba, East Nusa Tenggara, Indonesia. http://dx.doi.org/10.5716/WP16078.PDF

233. Central hypotheses for the third agroforestry paradigm within a common definition. http://dx.doi.org/10.5716/WP16079.PDF

234. Assessing smallholder farmers' interest in shade coffee trees: The Farming Systems of Smallholder Coffee Producers in the Gisenyi Area, Rwanda: a participatory diagnostic study. http://dx.doi.org/10.5716/WP16104.PDF

235. Review of agricultural market information systems in |sub-Saharan Africa. http://dx.doi.org/10.5716/WP16110.PDF

236. Vision and road map for establishment of a protected area in Lag Badana, Lower Jubba, Somalia. http://dx.doi.org/10.5716/WP16127.PDF

237. Replicable tools and frameworks for Bio-Carbon Development in West Africa. http://dx.doi.org/10.5716/WP16138.PDF

238. Existing Conditions, Challenges and Needs in the Implementation of Forestry and Agroforestry Extension in Indonesia. http://dx.doi.org/10.5716/WP16141.PDF

239. Situasi Terkini, Tantangan dan Kebutuhan Pelaksanaan Penyuluhan Kehutanan dan Agroforestri di Indonesia. http://dx.doi.org/10.5716/WP16142.PDF

240. The national agroforestry policy of India: experiential learning in development and delivery phases. http://dx.doi.org/10.5716/WP16143.PDF

241. Agroforestry and Forestry in Sulawesi series: Livelihood strategies and land-use system dynamics in Gorontalo. http://dx.doi.org/10.5716/WP16157.PDF

242. Seri Agroforestri dan Kehutanan di Sulawesi: Strategi mata pencaharian dan dinamika sistem penggunaan lahan di Gorontalo. http://dx.doi.org/10.5716/WP16158.PDF

243. Ruang, Gender dan Kualitas Hidup Manusia: Sebuah studi Gender pada komunitas perantau dan pengelola kebun di Jawa Barat. http://dx.doi.org/10.5716/WP16159.PDF

244. Gendered Knowledge and perception in managing grassland areas in East Sumba, Indonesia. http://dx.doi.org/10.5716/WP16160.PDF

245. Pengetahuan dan persepsi masyarakat pengelola padang aavana, Sebuah Kajian Gender di Sumba Timur. http://dx.doi.org/10.5716/WP16161.PDF

246. Dinamika Pengambilan Keputusan pada komunitas perantau dan pengelola kebun di Jawa Barat. http://dx.doi.org/10.5716/WP16162.PDF

247. Gaharu (eaglewood) domestication: Biotechnology, markets and agroforestry options. http://dx.doi.org/10.5716/WP16163.PDF

248. Marine habitats of the Lamu-Kiunga coast: an assessment of biodiversity value, threats and opportunities. http://dx.doi.org/10.5716/WP16167.PDF

249. Assessment of the biodiversity in terrestrial landscapes of the Witu protected area and surroundings, Lamu County Kenya. http://dx.doi.org/10.5716/WP16172.PDF

250. An ecosystem services perspective on benefits that people derive from biodiversity of Coastal forests in Lamu County, Kenya http://dx.doi.org/10.5716/WP16173.PDF 
251. Assessment of the biodiversity in terrestrial and marine landscapes of the proposed Laga Badana National Park and surrounding areas, Jubaland, Somalia.

http://dx.doi.org/10.5716/WP16174.PDF

2017

252. Preferensi Petani terhadap Topik Penyuluhan dan Penyebaran Informasi Agroforestri di Indonesia. http://dx.doi.org/10.5716/WP16181.PDF

253. Seri Agroforestri dan Kehutanan di Sulawesi: Keanekaragaman hayati jenis pohon pada hutan rakyat agroforestri di DAS Balangtieng, Sulawesi Selatan. http://dx.doi.org/10.5716/WP16182.PDF

254. Potensi dan Tantangan dalam Pengembangan Skema Ko-Investasi Jasa Lingkungan di Kabupaten Buol, Indonesia. http://dx.doi.org/10.5716/WP17008.PDF

255. Keragaman Jenis Pohon dan Pemanfaatannya oleh Masyarakat di Kabupaten Buol, Indonesia. http://dx.doi.org/10.5716/WP17009.PDF

256. Kerentanan dan preferensi sistem pertanian petani di Kabupaten Buol, Indonesia. http://dx.doi.org/10.5716/WP17010.PDF

257. Dinamika Perubahan Penggunaan/Tutupan Lahan Serta Cadangan Karbon di Kabupaten Buol, Indonesia. http://dx.doi.org/10.5716/WP17011.PDF

258. The Effectiveness of the Volunteer Farmer Trainer Approach vis-à-vis Other Information Sources in Dissemination of Livestock Feed Technologies in Uganda.

http://dx.doi.org/10.5716/WP17104.PDF

259. Agroforestry and Forestry in Sulawesi series: Impact of agricultural-extension booklets on community livelihoods in South and Southeast Sulawesi. http://dx.doi.org/10.5716/WP17125.PDF

260. Petani Menjadi Penyuluh, Mungkinkah? Sebuah Pendekatan Penyuluhan dari Petani ke Petani di Kabupaten Sumba Timur. http://dx.doi.org/10.5716/WP17145.PDF

261. Dampak Perubahan Tutupan Lahan terhadap Kondisi Hidrologi di Das Buol, Kabupaten Buol, Sulawesi Tengah: Simulasi dengan Model Genriver. http://dx.doi.org/10.5716/WP17146.PDF

262. Analisis Tapak Mata Air Umbulan, Pasuruan, Jawa Timur. Kajian elemen biofisik dan persepsi masyarakat. http://dx.doi.org/10.5716/WP17147.PDF

263. Planned comparisons demystified. http://dx.doi.org/10.5716/WP17354.PDF

264. Soil health decision support for NERC digital soil platforms: A survey report. http://dx.doi.org/10.5716/WP17355.PDF

265. Seri Pembangunan Ekonomi Pedesaan Indonesia: Menanam di bukit gundul: Pengetahuan masyarakat lokal dalam upaya restorasi lahan di Sumba

Timur. http://dx.doi.org/10.5716/WP17356.PDF

266. Tree diversity and carbon stock in three districts of Kutai Timur, Pasir and Berau, East Kalimantan http://dx.doi.org/10.5716/WP17357.PDF

267. Tree Diversity and Carbon Stock in Various Land Use Systems of Banyuasin and Musi Banyuasin Districts, South Sumatera http://dx.doi.org/10.5716/WP17358.PDF

268. Tree diversity and carbon stock in various land cover systems of Jayapura, Jayawijaya and Merauke Districts, Papua Province http://dx.doi.org/10.5716/WP17359.PDF

269. Modelling tree production based on farmers' knowledge: case for kapok (Ceiba pentandra) and candlenut (Aleurites mollucana) under various agroforestry scenarios.

http://dx.doi.org/10.5716/WP17361.PDF 
270. The Impact of Land Cover and Climate Change on Present and Future Watershed Condition. Study case: Tugasan, Alanib and Kulasihan Sub-watershed of Manupali Watershed, Lantapan, Bukidnon, Philippines. http://dx.doi.org/10.5716/WP17362.PDF

271. Tree Diversity and Above-ground Carbon Stock estimation in Various Land use Systems in Banjarnegara, Banyumas and Purbalingga, Central Java. http://dx.doi.org/10.5716/WP17363.PDF

272. Agroforestry and Forestry in Sulawesi series: Landscape Management Strategies in Sulawesi: Review of Intervention Options. http://dx.doi.org/10.5716/WP17364.PDF

273. Household Food-Security and Nutritional Status of Women and Children in Buol Regency, Central Sulawesi, Indonesia. http://dx.doi.org/10.5716/WP17365.PDF

274. Palm oil expansion in tropical forest margins or sustainability of production? Focal issues of regulations and private standards. http://dx.doi.org/10.5716/WP17366.PDF

2018

275. Decision analysis methods guide: agricultural policy for nutrition http://dx.doi.org/10.5716/WP18001.PDF

276. Supporting human nutrition in Africa through the integration of new and orphan crops into food systems: Placing the work of the African Orphan Crops Consortium in context. http://dx.doi.org/10.5716/WP18003.PDF

277. Seri Pembangunan Ekonomi Pedesaan Indonesia. Pilihan Manajemen Budidaya Kacang Tanah sebagai Upaya untuk Memperbaiki Penghidupan Masyarakat Haharu. http://dx.doi.org/10.5716/WP18004.PDF

278. Estudio de línea de base CCAFS a nivel de hogar en Nicaragua y Costa Rica. Fase de diagnóstico del estudio: "Contribución de la diversidad arbórea a los medios de vida para la adaptación y la mitigación al cambio climático. http://dx.doi.org/10.5716/WP18005.PDF

279. Understanding tree cover transition, drivers and stakeholder perspectives for effective landscape governance. A case study in Na Nhan commune, Dien Bien province, Vietnam. http://dx.doi.org/10.5716/WP18006.PDF

280. El Sistema "Quesungual": Agroforestería y manejo de suelos para la producción de maíz y frijol en laderas. http://dx.doi.org/10.5716/WP18007.PDF

281. Probabilistic Decision Modelling to Determine Impacts on Natural Resource Management and Livelihood Resilience in Marsabit County, Kenya. http://dx.doi.org/10.5716/WP18008.PDF

282. Shifting discourse, shifting power: how is climate change mitigation and justice negotiated in Indonesia? http://dx.doi.org/10.5716/WP18009.PDF

283. Result of Land Use Planning and Land Administration (LULA) Implementation in South Sumatra, East Kalimantan, Central Java and Papua. http://dx.doi.org/10.5716/WP18010.PDF

284. Farmers' preferences for training topics and dissemination of agroforestry information in Indonesia. http://dx.doi.org/10.5716/WP18015.PDF

285. CSA-Diagnostic (CSA-Dx): A primer for investigating the 'climate-smartness' of ag technologies. http://dx.doi.org/10.5716/WP18020.PDF

286. An analysis of the vulnerability of poor communities in Yunnan Province, China. http://dx.doi.org/10.5716/WP18021.PDF

287. Gendered space and quality of life: gender study of out-migration and smallholding agroforestry communities in West Java Province, Indonesia. http://dx.doi.org/10.5716/WP18024.PDF 
288 Evaluation of UTZ certification coffee businesses in Guatemala, Honduras and Nicaragua. http://dx.doi.org/10.5716/WP18028.PDF

289 Agroforestry species of Peru: annotated list and contribution to prioritization for genetic conservation. http://dx.doi.org/10.5716/WP18029.PDF

290 Indonesia Rural Economic Development Series. Growing plants on a barren hill: local knowledge as part of land restoration in Sumba Timur, Indonesia. http://dx.doi.org/10.5716/WP18030.PDF

291 Assessing the Downstream Socioeconomic Impacts of Agroforestry in Kenya. http://dx.doi.org/10.5716/WP18033.PDF

2019

292 Los árboles fuera del bosque en la NAMA forestal de Colombia. Elementos conceptuales para su contabilización. http://dx.doi.org/10.5716/WP19002.PDF

293 Gender and Adaptation: An Analysis of Poverty and Vulnerability in Yunnan, China. http://dx.doi.org/10.5716/WP19004.PDF

294 Tree cover on agricultural land in the Asia-Pacific region.

http://dx.doi.org/10.5716/WP19005.PDF 


The World Agroforestry Centre is an autonomous, non-profit research organization whose vision is a rural transformation in the developing world as smallholder households increase their use of trees in agricultural landscapes to improve food security, nutrition, income, health, shelter, social cohesion, energy resources and environmental sustainability. The Centre generates science-based knowledge about the diverse roles that trees play in agricultural landscapes, and uses its research to advance policies and practices, and their implementation that benefit the poor and the environment. It aims to ensure that all this is achieved by enhancing the quality of its science work, increasing operational efficiency, building and maintaining strong partnerships, accelerating the use and impact of its research, and promoting greater cohesion, interdependence and alignment within the organization.

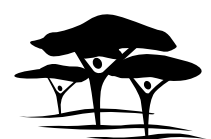

United Nations Avenue, Gigiri • PO Box 30677 • Nairobi, $00100 \cdot$ Kenya Telephone: +254 207224000 or via USA +1 6508336645 Fax: +254207224001 or via USA +1 6508336646

Email: worldagroforestry@cgiar.org•www.worldagroforestry.org

Southeast Asia Regional Program • Sindang Barang • Bogor 16680

PO Box $161 \cdot$ Bogor $16001 \cdot$ Indonesia

Telephone: +62 2518625415 • Fax: +62 2518625416

- Email: icraf-indonesia@cgiar.org

www.worldagroforestry.org/region/southeast-asia

blog.worldagroforestry.org 